FORSCHUNGSERGEBNISSE DER

Günther Sedlacek

\title{
Analyse der Studiendauer und des Studienabbruch-Risikos
}

unter Verwendung der statistischen Methoden der Ereignisanalyse 


\section{Analyse der Studiendauer und des Studienabbruch-Risikos}

Die Arbeit behandelt zunächst die statistische Theorie der Ereignisanalyse, die die Grundlage für die empirische Analyse der Studiendauer und des StudienabbruchRisikos auf der Basis von Studieneingangskohorten der Wirtschaftsuniversität Wien bildet. Die Einbeziehung erklärender Variablen in das statistische Modell und die Ermittlung des Effektes dieser Variablen auf die Dauer bis zum Eintreffen eines Ereignisses bilden einen Schwerpunkt der Arbeit. Es werden parametrische Regressionsmodelle zur Analyse von Verweildauern unter Einbeziehung von (auch zeitabhängigen) erklärenden Variablen und semiparametrische, proportionale Hazardraten-Modelle diskutiert und gegenübergestellt. Ferner werden die verfügbaren Studentendaten aus der Hörerevidenz und aus einer ergänzend durchgeführten Befragung deskriptiv analysiert und versucht, erste Zusammenhänge zwischen einzelnen Variablen und dem Studierstatus aufzuzeigen. Ein weiterer Schwerpunkt liegt in der Anwendung der zuvor beschriebenen parametrischen und semiparametrischen Mehr-Zustands-Modelle für die Analyse der Studiendauer und von Studienabbruch-Wahrscheinlichkeiten an der Wirtschaftsuniversität Wien.

Günther Sedlacek, geboren 1972 in Wien, studierte Technische Mathematik an der Technischen Universität Wien. Von 1997 bis 2001 war er als Wissenschaftlicher Mitarbeiter bei den Projekten Evaluierung der Lehre und Studienverlaufsanalyse und als Lektor an der Wirtschaftsuniversität Wien beschäftigt. Seit 2001 ist er als Angestellter der Oesterreichischen Nationalbank mit der Erstellung von volkswirtschaftlichen Statistiken im Zusammenhang mit Wertpapieren beschäftigt. 2003 promovierte er am Institut für Statistik der Wirtschaftsuniversität Wien. 
Analyse der Studiendauer und des Studienabbruch-Risikos 


\section{Forschungsergebnisse der Wirtschaftsuniversität Wien}

Band 4

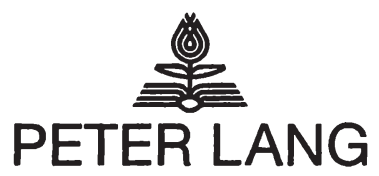

Frankfurt am Main · Berlin · Bern · Bruxelles - New York · Oxford · Wien 
Günther Sedlacek

\section{Analyse der Studiendauer und des Studienabbruch-Risikos}

unter Verwendung der statistischen Methoden der Ereignisanalyse

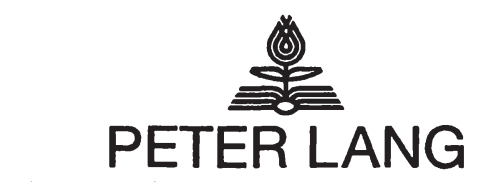

Europäischer Verlag der Wissenschaften 
Bibliografische Information Der Deutschen Bibliothek Die Deutsche Bibliothek verzeichnet diese Publikation in der Deutschen Nationalbibliografie; detaillierte bibliografische Daten sind im Internet über <http://dnb.ddb.de> abrufbar.

Open Access: The online version of this publication is published on www.peterlang.com and www.econstor.eu under the international Creative Commons License CC-BY 4.0. Learn more on how you can use and share this work: http://creativecommons. org/licenses/by/4.0.

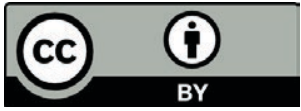

This book is available Open Access thanks to the kind support of ZBW - Leibniz-Informationszentrum Wirtschaft.

Zugl.: Wien, Wirtschaftsuniv., Diss., 2003

\author{
Gefordert durch die \\ Wirtschaftsuniversităt Wien.
}
Gedruckt auf alterungsbestăndigem, săurefreiem Papier. ISSN 1613-3056
ISBN3-631-52501-X
ISBN 978-3-631-75405-4 (eBook)
(C) Peter Lang GmbH
Europäischer Verlag der Wissenschaften
Frankfurt am Main 2004
Alle Rechte vorbehalten.

Das Werk einschließlich aller seiner Teile ist urheberrechtlich geschützt. Jede Verwertung außerhalb der engen Grenzen des

Urheberrechtsgesetzes ist ohne Zustimmung des Verlages unzulăssig und strafbar. Das gilt insbesondere für

Vervielfáltigungen, Übersetzungen, Mikroverfilmungen und die Einspeicherung und Verarbeitung in elektronischen Systemen.

Printed in Germany 123457

www.peterlang.de 
Mein Dank gilt insbesondere Prof. Peter Hackl für die intensive Betreuung dieser Arbeit und die gute Zusammenarbeit. Er ist mir stets - nicht nur für diese Arbeit - der richtige Wegweiser gewesen. Ebenso gilt mein Dank Prof. Hans Robert Hansen, der als Rektor die Finanzierung des Projektes Studienverlaufsanalyse unterstützt und somit den Weg für diese Dissertation geebnet hat, und der Wirtschaftsuniversität Wien, die die Veröffentlichung dieses $\mathrm{Bu}$ ches ermöglicht hat.

Schließlich gilt mein besonderer Dank noch meiner Frau Ela und meiner Mutter, die beide für das notwendige Umfeld gesorgt und es mir ermöglicht haben, die Dissertation und dieses Buch neben Beruf und Kinder fertig zu stellen.

Dieses Buch widme ich meinen beiden Töchtern Nicole und Vanessa. 
Günther Sedlacek - 978-3-631-75405-4

Downloaded from PubFactory at 01/11/2019 05:23:57AM

via free access 


\section{Inhaltsverzeichnis}

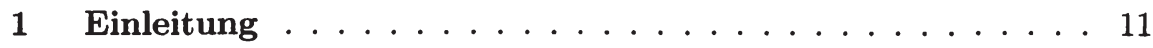

1.1 Hintergrund und Zielsetzungen der Arbeit . . . . . . . . . 11

1.2 Retrospektive und prospektive Methoden . . . . . . . . . . . 13

1.3 Aufbau und Gliederung . . . . . . . . . . . . . . . . . . 15

\section{A Statistische Theorie der Ereignisanalyse}

2 Einführung in die Ereignisanalyse . . . . . . . . . . 21

2.1 Ein Überblick über die Ereignisanalyse . . . . . . . . . . . . . 21

2.2 Ereignisdaten und Spezifika der Ereignisanalyse . . . . . . . . . 24

3 Grundkonzepte . . . . . . . . . . . . . . . . . . . . 29

3.1 Hazardrate, Überlebens- und Dichtefunktion . . . . . . . . . . . . 29

3.2 Zensieren und Abschneiden . . . . . . . . . . . . . . . . 31

4 Nicht-parametrische Verfahren . . . . . . . . . . . 36

4.1 Kaplan-Meier- und Nelson-Aalen-Methode . . . . . . . . . . . 36

4.2 Sterbetafel-Methode . . . . . . . . . . . . . . . . . . . . . 39

4.3 Vergleich von Überlebensfunktionen . . . . . . . . . . . . . . . . 40

5 Parametrische Regressionsmodelle . . . . . . . . . . . . 42

5.1 Typische Verteilungsannahmen und Einbeziehung von Kovariablen . 43

5.2 Schätzen der Parameter und Hypothesentests . . . . . . . . . 53 
5.3 Überprüfung von Verteilungsannahmen . . . . . . . . . . . . . . 57

5.4 Das stückweise konstante Hazards-Modell . . . . . . . . . . . . . 60

6 Das semiparametrische Hazards-Modell von Cox . . . . . . 64

6.1 Hazardrate und Überlebensfunktion im Cox-Modell . . . . . . . . . 65

6.2 Partielle Likelihood Schätzung . . . . . . . . . . . . . 66

6.3 Zeitabhängige Kovariablen . . . . . . . . . . . . . . . 69

6.4 Das stratifizierte Cox-Modell . . . . . . . . . . . . . 76

6.5 Modellüberprüfungen . . . . . . . . . . . . . 78

7 Mehr-Zustands- und Mehr-Episoden-Modelle . . . . . . . . 84

7.1 Mehr-Zustands-Modelle . . . . . . . . . . . . . . . . 84

7.2 Erweiterung der Mehr-Zustands-Modelle auf den Mehr-EpisodenFall . . . . . . . . . . . . . . . . . . . 90

8 Diskrete Ereignisdatenmodelle . . . . . . . . . . . . 95

8.1 Logistisches- und gruppiertes Cox-Modell . . . . . . . . . . . 96

8.2 Maximum-Likelihood-Schätzung . . . . . . . . . . . . . 98

B Empirische Analyse der Studiendauer und des Studienabbruch-Risikos

9 Studien zu den Themen Studiendauer und Studienabbruch . . . . . . . . . . . . . . . . . 103

9.1 Befragungen . . . . . . . . . . . . . . 103

9.2 Analyse der Daten aus der Hörerevidenz . . . . . . . . . . . 107

9.3 Studien untzer Verwendung der Ereignisdatenanalyse . . . . . . 110

10 Daten und erklärende Variablen . . . . . . . . . . . . 113

10.1 Daten aus der Hörerevidenz (Systemdaten) . . . . . . . . . . . 114 
10.2 Ergänzende Daten aus einer Befragung . . . . . . . . . . . . 122

11 Deskriptive Analysen . . . . . . . . . . . . . 127

11.1 Beschreibung des Studienfortschritts . . . . . . . . . . . 127

11.2 Analyse des Studienfortschritts in Abhängigkeit von demographischen Variablen . . . . . . . . . . . . . . . 133

12 Analyse der Studiendauer und der Studienabschlussrate mit Hilfe von Ereignisdatenmodellen . . . . . . . . . . 147

12.1 Einleitung . . . . . . . . . . . . . . . 147

12.2 Analyse der Studiendauer - log-lineare parametrische Modelle . . . 148

12.3 Analyse der Studienabschlussrate - semiparametrische Modellierung . . . . . . . . . . . . . . 168

13 Analyse des Studienabbruchs . . . . . . . . . . 185

13.1 Einleitung . . . . . . . . . . . . . . . . 185

13.2 Analyse der Studienabbruch-Wahrscheinlichkeit ohne positive Leistung . . . . . . . . . . . . . . . . 186

13.3 Analyse der Studienabbruchrate . . . . . . . . . . . . . 192

14 Erweiterung der Analysen . . . . . . . . . . . . . 199

14.1 Sensitivitätsanalyse . . . . . . . . . . . . . . . 199

14.2 Mehr-Episoden-Modelle . . . . . . . . . . . . . . . 201

14.3 Diskrete Modelle . . . . . . . . . . . . . . . . . . . . . 202

15 Zusammenfassung und Diskussion der Ergebnisse . . . . . 204

15.1 Analyse der Studiendauer und der Hazardrate für den Studienabschluss . . . . . . . . . . . . . . . . . . . 204

15.2 Analyse der Studienabbruchrate . . . . . . . . . . 206

15.3 Ausblick . . . . . . . . . . . . . . . . 207

Anhang A ............................... 209

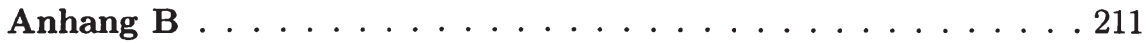


Abbildungsverzeichnis . . . . . . . . . . . . . . . 217

Tabellenverzeichnis . . . . . . . . . . . . . . . . 219

Literaturverzeichnis . . . . . . . . . . . . . . . 223 


\section{Kapitel 1}

\section{Einleitung}

\subsection{Hintergrund und Zielsetzungen der Arbeit}

Seit Jahren sorgen überlange Studienzeiten und hohe Abbruchraten für Diskussionsstoff an den Universitäten. An der Wirtschaftsuniversität Wien (WU) wird im Kenndaten-Bericht 2001 retrospektiv für den Absolventenjahrgang 1999/2000 (Absolventen im Zeitraum vom 1. Oktober 1999 bis 30. September 2000) eine durchschnittliche Dilpomstudiendauer von etwa 14.8 Semester angegeben; die vorgeschriebene Studiendauer ist 8 Semester. Über die Abbruchquote von Studierenden der Wirtschaftsuniversität gibt es seitens der Universitätsleitung nur grobe Schätzungen; jedoch liegt die Zahl derer eines Jahrgangs, die das Studium beenden, weit unter der Zahl derer, die das Studium beginnen. Diese Zahlen sind Anlass für die Durchführung von Studien über Ursachen und Gründe von langen Studienzeiten und hohen Abbruchraten. Diese Studien werden meist in Form von Befragungen von Studienabbrechern und Langzeitstudierenden durchgeführt. Daneben bieten auch die Daten aus der Hörerevidenz, der universitätsinternen Dokumentation der Studienverlaufsdaten der Studierenden, ein großes Informationspotential. Universitäten verfügen in ihren Datenbanken über genaue Daten über die inskribierten Studien und (inneruniversitären) Prüfungsaktivitäten sowie über Daten zu demographischen Variablen ihrer Studierenden wie beispielsweise Schultyp, Geschlecht und Nationalität. Die Analyse der Daten aus der Hörerevidenz und aus Befragungen ermöglicht die Bereitstellung von differenzierter und verlässlicher Information über

1. die Studiendauer,

2. den Studienabbruch, 


\section{3. den Studienverlauf und}

4. das Studienverhalten.

Eine nähere Auseinandersetzung mit dem Thema zeigt die Komplexität der Begriffe Studiendauer und Studienabbruch. Eine exakte Definition dieser beiden Begriffe ist Voraussetzung und wichtiger Bestandteil einer ernst zu nehmenden Analyse.

Der Bedarf seitens der Universitätsleitung an differenzierter Information zu den oben genannten Punkten, aber auch das Interesse der Universitätsangehörigen und vieler Aussenstehender ist Motivation, Analysen des Studienerfolgs und des -abbruchs durchzuführen. Daneben gewinnen umfassende Analysen zu diesem Thema auch im Hintergrund von Diskussionen um die Autonomie von Universitäten zusehends an Bedeutung. In einigen europäischen Ländern sind Leistungskennzahlen (engl. performance indicators) für die Universitäten eingeführt worden, die neben der Zahl der Studienabschlüsse auch die Abbruchquote als einen Indikator enthalten. An diesen Leistungskennzahlen orientiert sich die Aufteilung des zur Verfügung stehenden Budgets. Analysen des Studienabbruchs etwa können dazu beitragen, Einflussgrößen auf die Abbruchraten zu identifizieren, und machen deutlich, inwieweit die einbezogenen Variablen Unterschiede in den Abbruchraten erklären; siehe Smith und Naylor (2001).

Die vorliegende Arbeit ist im Rahmen des Projektes "Studienverlaufsanalyse" entstanden (Hackl und Sedlacek (2002)). Die Zielsetzungen des Projektes, das von der Universitätsleitung in Auftrag gegeben worden ist, können in die folgenden drei Punkte zusammengefasst werden:

- Umfassende empirische Analyse der Studiendauer und des StudienabbruchRisikos von WU-Studierenden - in Abhängigkeit von

- studienerfolgsbezogenen Merkmalen (wie etwa der Anzahl der Leistungen im ersten Studienjahr)

- studentenbezogenen Merkmalen aus der Hörerevidenz (wie Geschlecht, Schultyp oder Alter)

- studentenbezogenen Variablen, die nicht in der WU-Studenten-Datenbank STEP verfügbar sind (wie dem zeitlichen Verlauf einer Erwerbstätigkeit parallel zum WU-Studium)

- Definition und Berechnung von Kennzahlen, die über den Studienverlauf Bescheid geben und derzeit nicht zur Verfügung stehen; im Detail wurde 
- die Studiendauer von Absolventenjahrgängen (und Teilpopulationen) analysiert,

- die Dauer bis zum Studienabbruch von Studienabbrechern eines Studienjahres geschätzt, sowie

- der Studienerfolg und -fortschritt von Studieneingangs-Kohorten behandelt.

- Konzeption und Entwicklung eines Instruments, das die routinemäßige Durchführung der im vorangegangenen Punkt einmalig durchgeführten Analysen erlaubt.

\subsection{Retrospektive und prospektive Methoden}

Die statistischen Methoden, die zum Erreichen der oben angeführten Zielsetzungen herangezogen wurden, sind (numerische und grafische) Verfahren der deskriptiven Statistik (Häufigkeitsverteilungen, Lagemaße einer Verteilung und Kreuztabellen für die Analyse des Zusammenhangs von zwei Merkmalen) sowie Methoden, die in der Theorie der Ereignisanalyse entwickelt wurden. Abhängig von der Fragestellung wurden

- (retrospektive) Analysen von Absolventen- bzw. Abbrecherjahrgängen, bzw.

- (prospektive) Analysen von Studienanfänger-Jahrgängen (StudieneingangsKohorten) durchgeführt.

Retrospektive Analysen wurden mit folgendem Ziel durchgeführt:

- Schätzung der Verteilung der Studiendauer von Absolventen- und von Abbrecherjahrgängen und Subpopulationen der Jahrgänge; diese Subpopulationen können durch Unterscheidungen der Absolventen (bzw. Studienabbrecher) nach Studienrichtung, Geschlecht, Schulform, etc. gebildet werden.

Prospektive Analysen verfolgten das Ziel der

- Schätzung der Studiendauer und der Studienabbruch-Wahrscheinlichkeit von (Teilpopulationen von) Studienanfänger-Jahrgängen, sowie der

- Analyse des Effekts von potentiellen Einflussgrößen auf die Studiendauer bzw. das Studienabbruch-Risiko. 
Bei der Analyse von Studienanfänger-Jahrgängen werden Erstinskribenten bis

a. zu ihrem erfolgreichen Studienabschluss

b. zu ihrem Studienabbruch

c. bis zum Ende der Studie (Stichtag) verfolgt.

Der Fall (c) tritt bei Studierenden ein, die zum Stichtag das Studium weder abgeschlossen noch abgebrochen haben. Vorteile der Analyse von StudieneingangsKohorten gegenüber retrospektiven Analysen sind etwa:

- In der Analyse der Studiendauer in Abhängigkeit von potentiellen Einflussgrößen werden nicht nur Absolventen sondern auch Studienabbrecher einbezogen.

- Angehörige desselben Immatrikulationsjahrgangs studieren unter den selben Rahmenbedingungen.

- Die Überprüfung von außeruniversitären Maßnahmen (wie der Einführung eines Leistungsnachweises für die Familienbeihilfe oder der Einführung von Studiengebühren) und von inneruniversitären Maßnahmen zur Studienzeitverkürzung (etwa dem WU-Projekt "Studieren in Teams", das die Studieneingangsphase erleichtern und somit die Studiendauer verkürzen helfen soll) ist durch Vergleich von Kohorten möglich.

- Durch Teilung des Studiums in einzelne Phasen (erstes Studienjahr bzw. Studieneingangsphase, erster Abschnitt) können schon früh Vergleiche mit früheren Kohorten gezogen und erste Aussagen über die "Performance" des "aktuellen" Jahrgangs und Wirkung von getroffenen Maßnahmen gemacht werden.

In der vorliegenden Arbeit werden Studienanfänger-Jahrgänge analysiert. Es wird herausgearbeitet, dass sich zur Schätzung der Studiendauer und der Abbruchwahrscheinlichkeit von Studienanfänger-Jahrgängen sowie zur Analyse des Effekts von potentiellen Einflussgrößen auf die Studiendauer bzw. das Studienabbruch-Risiko speziell die statistischen Methoden der Ereignisanalyse eignen.

Mit Hilfe dieses statistischen Verfahrens soll am Beispiel der Wirtschaftsuniversität Wien (entsprechend den angeführten Zielsetzungen) die Beantwortung folgender beispielhafter Fragestellungen möglich sein: 
- Welche (zu Studienbeginn gegebenen) Merkmale (wie Schultyp, Schulnoten, Berufstätigkeit zu Studienbeginn oder Alter) beeinflussen die Studiendauer bzw. das Studienabbruch-Risiko?

- Sind die Leistungen im ersten Studienjahr prädiktiv für die Studiendauer und die Wahrscheinlichkeit eines Studienabbruchs? Welche Zusammenhänge bestehen zwischen einzelnen Studienphasen?

- Führen Veränderungen in dynamischen Variablen (wie der Berufsintensität, dem Betreiben einer zweiten Ausbildung oder der Betreuung eines Kindes) zu Verzögerungen im Studium?

- Sind Unterschiede in der Erklärung von frühen und späten Studienabbrüchen zu finden?

\subsection{Aufbau und Gliederung}

Die Arbeit ist in zwei Teile unterteilt. Der erste Teil behandelt die statistische Theorie der Ereignisanalyse, die die Grundlage für den zweiten, empirischen Teil bildet. Ereignisanalysen haben in den letzten beiden Jahrzehnten in vielen Bereichen und in der statistischen Literatur zunehmend an Bedeutung gewonnen (siehe Abschnitt 2.1), ihre Grundkonzepte und Methoden sind (im Gegensatz zur linearen Regression oder zur Analyse von Kontigenztafeln etwa) jedoch kaum in Grundlagenbüchern der Statistik und der empirischen Sozialforschung zu finden; eine Ausnahme in der deutschsprachigen Literatur bilden beispielsweise die Statistik-Lehrbücher von Toutenburg (2000a,b).

Mit dem Begriff Ereignisanalyse werden statistische Verfahren zur Untersuchung von Zeitintervallen zwischen aufeinanderfolgenden Ereignissen bezeichnet. Die wichtigsten Konzepte werden im ersten Teil überblicksmäßig erläutert und sollen den Leser mit den Methoden der Ereignisanalyse vertraut machen. Das Hauptaugenmerk liegt auf der Behandlung von Problemstellungen, die für die Analyse von Studienverläufen typisch sind. Zahlreiche Literaturhinweise zu den jeweiligen Abschnitten ermöglichen eine vertiefende Analyse der präsentierten Methoden.

Im Detail wird im ersten Teil auf folgende Themen eingegangen:

- Kapitel 2 setzt sich im ersten Abschnitt mit der zunehmenden Bedeutung der Ereignisanalyse auseinander, die sich in den vielfältigen Anwendungsbereichen, in der Verfügbarkeit vieler Methoden in der kommerziellen 
Statistik-Software (wie zum Beispiel SAS, S-PLUS, BMDP und SPSS) und in den in den letzten Jahren zahlreich erschienenen Monographien zum Thema verdeutlicht. Der zweite Abschnitt geht auf die Spezifika der Ereignisanalyse ein und erläutert, warum Ereignisdaten eigene Analyse-Methoden verlangen.

- Kapitel 3 befasst sich im ersten Abschnitt mit Funktionen, die zur Beschreibung von Ereignisdaten verwendet werden - nämlich mit der Hazard- und der Überlebensfunktion. Im zweiten Abschnitt werden zensierte und abgeschnittene Daten erläutert; das sind unvollständige Daten, die für Problemstellungen der Ereignisanalyse und besonders für Studienverlaufsanalysen typisch sind.

- Kapitel 4 diskutiert gängige nicht-parametrische Verfahren zur Analyse von Verweildauern - die Kaplan-Meier-, die Nelson-Aalen- und die SterbetafelMethode. Diese Verfahren eigenen sich vor allem zur explorativen Analyse von Ereignisdaten. Nicht-parametrische Methoden zum Vergleich der Überlebensfunktionen von Teilpopulationen werden im letzten Abschnitt erläutert.

- Die Einbeziehung erklärender Variablen in das statistische Modell und die Ermittlung des Effektes dieser Variablen auf die Dauer bis zum Eintreffen eines Ereignisses sind für die statistische Analyse von großer Bedeutung und auch im zweiten Teil dieser Arbeit die zentrale Aufgabenstellung.

Kapitel 5 diskutiert parametrische Regressionsmodelle zur Analyse von Verweildauern unter Einbeziehung von erklärenden Variablen. Parametrische Modelle erfordern Verteilungsannahmen für die Zeitdauer. Im ersten Abschnitt des Kapitels werden typische Verteilungsannahmen wie das Exponential- und Weibull-Modell beschrieben. Der zweite Abschnitt geht auf die Schätzung der Parameter dieser Variablen ein. Im dritten Abschnitt werden Methoden zur Überprüfung der Verteilungsannahmen behandelt. Der letzte Abschnitt dieses Kapitels setzt sich mit dem stückweise konstanten Exponential-Modell auseinander, das eine Verallgemeinerung des einfachen Exponential-Modells ist, jedoch die Daten zumeist weitaus besser beschreibt und daher in Anwendungsfällen häufig verwendet wird.

- Kapitel 6 setzt sich mit dem semiparametrischen proportionalen Modell von Cox auseinander. Dieses Modell wird häufig in angewandten Analysen verwendet, da es keine Verteilungsannahme für die Zeitdauer verlangt und somit flexibler als parametrische Regressionsmodelle ist. Im ersten Abschnitt werden die grundlegenden Eigenschaften des Modells erläutert, etwa 
die Annahme von proportionalen Hazardraten zweier Individuen mit unterschiedlichen Ausprägungen in den (zeitunabhängigen) Kovariablen; der zweite Abschnitt behandelt die gegenüber parametrischen Modellen modifizierte Schätzung der Modell-Parameter. Der darauffolgende Abschnitt diskutiert die Einbeziehung von zeitabhängigen Kovariablen in semiparametrische und parametrische Ereignisdatenmodelle. Danach wird das stratifizierte Cox-Modell behandelt, das etwa bei Verletzung der Proportionalitätsannahme verwendet wird. Modellüberprüfungen mit Hilfe von Residuen sind das Thema des letzten Abschnitts.

- In den beiden darauffolgenden Kapiteln 7 und 8 werden die in den Kapiteln 3 bis 6 für kontinuierliche Modelle mit einem Anfangszustand und einem (absorbierenden) Endzustand präsentierten Methoden auf den Mehr-Zustandsund Mehr-Episoden-Fall (Kapitel 7) und auf diskrete Modelle (Kapitel 8) erweitert.

Der zweite Teil umfasst die empirische Analyse der Studiendauer und des Studienabbruch-Risikos auf der Basis von Studieneingangs-Kohorten der Wirtschaftsuniversität. Mit Hilfe der im ersten Teil beschriebenen Methoden der Ereignisanalyse werden bestimmte Fragestellungen zu Studiendauer und -abbruch beantwortet.

- Kapitel 9 setzt sich einleitend mit (nationalen und internationalen) Studien zu den Themen Studiendauer und Studienabbruch auseinander. Dabei wird zwischen Arbeiten unterschieden, in denen Daten aus der Hörerevidenz oder aus Befragungen analysiert worden sind. Im Abschnitt 9.3 wird auch auf zwei Arbeiten eingegangen, in denen die Studiendauer und das Studienabbruch-Risiko mit Hilfe von Ereignisdatenmodellen analysiert worden sind.

- Kapitel 10 erläutert im ersten Abschnitt, welche Daten in der StudentenDatenbank der Wirtschaftsuniversität in welcher Form zur Verfügung stehen. Im darauffolgenden Abschnitt wird das Design und der Inhalt der durchgeführten Befragung (zur Erhebung ergänzender Variablen) behandelt und auf Probleme der Datenaufbereitung eingegangen.

- Das darauffolgende Kapitel 11 analysiert die verfügbaren Daten deskriptiv und versucht, (in Form von Kreuztabellen) erste Zusammenhänge zwischen einzelnen Variablen und dem Studierstatus aufzuzeigen.

- Die Kapitel 12 und 13 bilden den Schwerpunkt des zweiten Teils der Arbeit. In den beiden Kapiteln werden die im ersten Teil beschriebenen Verfahren insbesondere parametrische und semiparametrische Mehr-Zustands-Modelle 
- auf die in den vorangegangenen Kapiteln erläuterten Daten angewendet und spezifische Fragestellungen zu Studiendauer und -abbruch beantwortet.

- Im Kapitel 14 wird auf weitere Analysemöglichkeiten der vorliegenden Daten eingegangen. So kommen etwa Modelle zur Anwendung, mit denen auch einzelne Studienphasen (Studieneingangsphase, erster und zweiter Studienabschnitt) getrennt voneinander bzw. in Abhängigkeit zueinander analysiert werden können.

- Das letzte Kapitel fasst die wichtigsten Ergebnisse zusammen. 


\section{Teil A \\ Statistische Theorie der Ereignisanalyse}


Günther Sedlacek - 978-3-631-75405-4

Downloaded from PubFactory at 01/11/2019 05:23:57AM

via free access 


\section{Kapitel 2}

\section{Einführung in die Ereignisanalyse}

Dieses einführende Kapitel soll dem Leser typische Anwendungsmöglichkeiten und Problemstellungen der Ereignisanalyse näher bringen. Es wird erläutert, was Ereignisdaten sind, wie sie erhoben und mit welchen Zielsetzungen Ereignisanalysen meist durchgeführt werden. Anhand der Spezifika der Ereignisanalyse - die Berücksichtigung von zensierten Daten und zeitabhängigen Variablen - wird erklärt, warum Ereignisdaten ihre eigenen Analyse-Methoden verlangen und nur mit (großem) Informationsverlust mit "klassischen" Methoden wie logistische und lineare Regression behandelt werden können.

\subsection{Ein Überblick über die Ereignisanalyse}

\section{Entwicklung der Methoden in verschiedenen Anwen- dungsfeldern}

Die Ereignisanalyse bezeichnet eine Klasse von statistischen Verfahren, die Zeitdauern zwischen aufeinanderfolgenden Zustandswechseln bzw. Ereignissen untersuchen. Diese Verfahren haben in den letzten beiden Jahrzehnten zunehmend an Bedeutung gewonnen und in verschiedensten Gebieten ihre Anwendung gefunden. Hosmer und Lemeshow (1999) meinen zu den jüngsten Entwicklungen auf diesem Gebiet: "It is difficult to find either a subject matter or a statistical journal that does not have at least one paper devoted to use or development of these methods". Auch die Gründung der Zeitschrift Lifetime Data Analysis im Jahr 1995, die ausschließlich den statistischen Methoden 
und Anwendungen der Ereignisanalyse gewidmet ist, widerspiegelt die zunehmende Bedeutung dieser Methoden. Die Herausgeber der Zeitschrift nennen als Anwendungsgebiete der Ereignisanalyse beispielsweise: actuarial science, economics, engineering sciences, environmental sciences, management science, medicine, operations research, public health, social and behavioral sciences. Die zahlreichen Anwendungsfelder, in denen sich diese Verfahren oft unabhängig entwickelt haben, sind auch der Grund, warum die gleichen statistischen Grundkonzepte - je nach Anwendungsgebiet und Fragestellung - unter verschiedenen (englischen) Namen geführt werden. Allison (1998) listet die Begriffe Survival Analysis (Medizin), Event History Analysis (Sozial- und Wirtschaftswissenschaften), Reliability Analysis, Duration Analysis (beide in den Naturwissenschaften) und Transition Analysis (Volkswirtschaft) auf.

Am häufigsten wird in der englischsprachigen Literatur der Begriff Survival Analysis verwendet, da die Methoden der Ereignisanalyse ursprünglich vor allem in klinischen Studien zur Analyse der Überlebenszeit von Patienten nach einer bestimmten Therapie eingesetzt worden sind. Die Medizin stellt bis heute ein sehr breites Anwendungsfeld der Ereignisanalyse dar, jedoch wird der Begriff Survival Analysis auch bei nicht-medizinischen Anwendungen und MehrEpisoden-Modellen verwendet.

Auch im deutschsprachigen Raum werden die Begriffe Ereignis(daten)analyse, Verlaufs(daten)analyse und Lebensdauer- bzw. Überlebensanalyse für die gleichen statistischen Grundkonzepte verwendet, wobei der Begriff Lebensdauerbzw. Überlebensanalyse meist nur für Ein-Episoden-Modelle benutzt wird. In dieser Arbeit wird der Begriff Ereignis(daten)analyse verwendet.

Häufige Anwendungsbeispiele aus der Sozialforschung sind:

- Studien zum Thema Beschäftigung; hier interessiert beispielsweise die Zeitdauer bis zum Wechsel oder Verlust des Arbeitsplatzes, bis zur Beförderung innerhalb einer Firma oder die Dauer der Arbeitslosigkeit

- Demographische Studien, in denen zum Beispiel das Lebensalter von Frauen bei Geburt ihres ersten Kindes, das Lebensalter bei der ersten Heirat oder die Dauer von Ehen untersucht wird; sowie

- Studien zur "Überlebensdauer" von Unternehmen, der Dauer bis zum Zusammenbruch eines politischen Systems

In dieser Arbeit ist die Studiendauer, das heißt die Dauer bis zum erfolgreichen Studienabschluss bzw. bis zum -abbruch, von Interesse. 


\section{Literaturüberblick mit Schwerpunkt auf anwendungsori- entierten Monographien}

Auch die kommerzielle Statistik-Software hat der wachsenden Bedeutung dieser Verfahren Rechnung getragen und erlaubt mittlerweile die Anwendung der gängigen Methoden der Ereignisanalyse. Harrell und Goldstein (1997) vergleichen die wichtigsten kommerziellen Statistik-Software Angebote bezüglich ihrer Anwendungsmöglichkeiten für Ereignisanalysen. Allison (1998) erläutert ausführlich die Anwendung der vorgestellten Verfahren (anhand von Datensätzen aus verschiedenen Bereichen) mit Hilfe von SAS; Klein und Moeschberger (1997) geben in einigen praktischen Hinweisen am Ende eines jeden Abschnitts an, welche Statistik-Software sich zur Durchführung der vorgestellten Verfahren eignet, und Kleinbaum (1996) gibt anhand von Beispielen Hinweise zur Anwendung von SPIDA, SAS und BMDP; die behandelten Datensätze in den beiden letztgenannten Werken entstammen dem medizinischen Bereich. Auch die in den letzten Jahren zahlreich erschienenen (angewandten) Monographien zum Thema (zum Beispiel Collett (1994), Harrell (1997), Hosmer und Lemeshow (1999), Le (1997), Lee (1992), Yamaguchi (1991)) enthalten Hinweise zur Lösung der präsentierten Beispiele (meist aus dem medizinischen Bereich) mit kommerzieller Statistik-Software.

G. Rowher entwickelte zur Anwendung der Ereignisanalyse die Software TDA (Transition Data Analysis), die über Internet frei zugängig ist (http://www.stat. ruhr-uni-bochum.de/tda.html); das Handbuch zu dieser Software von Rohwer und Pötter (1998), das ausführliche Erläuterungen der dazugehörigen statistischen Theorie enthält, kann ebenfalls im Internet heruntergeladen werden. In Blossfeld und Rohwer (1995) werden einerseits Ereignisdatenmodelle zur kausalen Analyse von sozialen Prozessen erläutert, andererseits wird die Verwendung der Software TDA zur Analyse von Ereignisdaten demonstriert. Weitere angewandte Monographien - schwerpunktmäßig mit Beispielen aus der sozialwissenschaftlichen Forschung - sind Allison (1984) und Blossfeld et al. (1986).

Zur steigenden Bedeutung der Ereignisanalyse hat auch die Theorie der Zählprozesse (engl. counting processes) und der Martingale beigetragen. Die Anwendung dieser Theorie ermöglichte - auf relativ einfache Weise - die Untersuchung der statistischen Eigenschaften der nicht-parametrischen und parametrischen Methoden der Ereignisdatenanalyse, die vorher zum Teil nur auf sehr komplexe Weise oder gar nicht untersucht wurden, und rechtfertigt somit die in der Ereignisanalyse verwendeten Verfahren der schließenden Statistik. Eine detaillierte Darstellung dieser Theorie und der Resultate, die durch ihre Anwendung für die Ereignisdatenanalyse erzielt worden sind, können interessierte Leser in Fleming und Harrington (1991) und Andersen et al. (1993) nachlesen; 
Hosmer und Lemeshow (1999) sowie Klein und Moeschberger (1997) geben einen kurzen Überblick über diese Theorie und führen laufend an, welche Resultate auf die Theorie der Zählprozesse zurückzuführen sind, deren Herleitung detailliert in einem der beiden oben erwähnten Bücher nachgelesen werden kann.

Daneben zählen folgende Monographien zu Standardwerken der Ereignisdatenanalyse: Cox und Oakes (1984), Kalbfleisch und Prentice (1980), Lancaster (1990), Lawless (1982) sowie Tuma und Hannan (1984). Einen kurzen Überblick über die Grundkonzepte und Spezifika der Ereignisanalyse geben zum Beispiel Hougaard (1999a) und Petersen (1990).

\subsection{Ereignisdaten und Spezifika der Ereignis- analyse}

\section{Erheben von Ereignisdaten}

Ereignisdaten informieren für jede Untersuchungseinheit (Individuum, Firma, technisches Gerät etc.) über die Länge des Zeitintervalls bis zum Eintreffen eines Ereignisses, das den Übergang zwischen Zuständen beschreibt und daher auch als Zustandswechsel bezeichnet wird. Die Dauer bis zum Eintreffen des Ereignisses wird im statistischen Modell repräsentiert durch eine nicht-negative Zufallsvariable $T$. Die Zustandsvariable, die oft auch als Zustandsraum bezeichnet wird, kann endlich viele Ausprägungen besitzen und stellt somit eine diskrete Variable dar. Ein Beispiel für eine Zustandsvariable ist der Studierstatus. Es kann zwischen laufenden, abgeschlossenen und abgebrochenen Studien unterschieden werden. Der Studienabschluss erklärt den Zustandswechsel vom Studierenden zum Absolventen. Ein weiteres Beispiel ist die Variable Familienstand mit den Ausprägungen ledig, verheiratet, geschieden und verwitwet. Das Ereignis (erste) Heirat beschreibt etwa den Übergang vom Zustand ledig in den Zustand verheiratet.

Zur Erhebung ereignisorientierter Daten eignet sich am besten ein prospektiver Beobachtungsplan, in dem Untersuchungseinheiten, die ein bestimmtes Ereignis in einem gewissen Zeitraum erlebt haben, vom Zeitpunkt dieses Ereignisses (dem Ausgangszeitpunkt bzw. Beginn der Beobachtungsperiode) bis zu einem Zustandswechsel oder bis zum Ende des Beobachtungszeitraums verfolgt werden. Ereignisse, die den Beginn der Beobachtungsperiode definieren, sind beispielsweise der Beginn eines Universitätsstudiums, die Entlassung aus dem Gefängnis oder die Diagnose einer bestimmten Krankheit. Je nach Frage- 
stellung werden die Untersuchungseinheiten im ersten Fall etwa bis zum Studienabschluss oder -abbruch, im zweiten Fall bis zum Rückfall der Straftäter und im dritten bis zum Tod verfolgt. Der Beginn der Beobachtungsperiode gibt den Zeitpunkt an, ab dem bekannt ist, dass die Untersuchungseinheiten dem Risiko (oder der Chance) ausgesetzt sind, dass das interessierende Ereignis eintrifft. Diese Periode wird daher auch oft als Risikoperiode bezeichnet, und die Menge aller Untersuchungseinheiten, die zu einem gewissen Zeitpunkt $t$ dem Risiko eines Ereignisses ausgesetzt sind, wird als Risikomenge $R(t)$ zum Zeitpunkt $t$ bezeichnet. Untersuchungseinheiten, die zu Studienende noch der Risikomenge angehören, nennt man rechts-zensiert. Diese Untersuchungseinheiten liefern die Information, dass das betrachtete Ereignis bis zum Ende der Beobachtungsperiode nicht eingetroffen ist, und werden in den Schätzverfahren der Ereignisanalyse berücksichtigt; siehe Abschnitt 3.2.

Prospektive Beobachtungspläne sind häufig sehr zeit- und kostenaufwendig, da es lange dauern kann, bis eine genügend große Anzahl von Ereignissen beobachtet worden ist. Aus diesem Grund werden häufig retrospektive Erhebungen von ereignisorientierten Daten in Form von Befragungen durchgeführt. Bei diesen Beobachtungsplänen werden Individuen rückwirkend nach dem Eintreffen der interessierenden Ereignisse gefragt. Bei retrospektiven Datenerhebungen treten vor allem zwei Probleme auf:

- Die befragten Individuen wissen eventuell nicht mehr die genauen Zeitpunkte, wann die interessierenden Ereignisse eingetroffen sind; sie können den exakten Verlauf nicht mehr rekonstruieren.

- Retrospektive Studien berücksichtigen nicht die Daten jener Individuen, die zum Befragungszeitpunkt nicht befragt werden konnten, weil sie beispielsweise gestorben, ausgewandert oder aus sonstigen Gründen nicht erreichbar sind.

In retrospektiv durchgeführten Lebensverlaufs- bzw. Berufsverlaufsstudien kommen beide Probleme zu tragen; vgl. Blossfeld und Rohwer (1995). Aber auch in Studienverlaufsanalysen ist man mit diesem Problem konfrontiert; siehe Abschnitt 10.2. Manchmal besteht die Möglichkeit, die meist lückenlos vorhandenen Daten aus administrativen Datenbanken - zum Beispiel von Universitäten oder Firmen - zu verwenden.

Ereignisdatenanalysen werden in der Regel durchgeführt, um den Einfluss von bestimmten Variablen auf die Dauer bis zum Eintreffen des interessierenden Ereignisses zu ermitteln. Diese Variablen können zeitkonstant sein, wie zum Beispiel das Geschlecht oder die Rasse, oder sich mit der Zeit ändern, wie 
der Familienstand oder das Einkommen. Bei zeitveränderlichen Variablen tritt bei retrospektiven Beobachtungsplänen, beispielsweise bei (retrospektiven) Lebensverlaufsstudien, auch häufig das Problem auf, dass sich die Befragten nicht mehr genau an den Verlauf dieser Variablen erinnern. In Studienverlaufsanalysen ist anzunehmen, dass die Intensität der Erwerbstätigkeit neben dem Studium einen Einfluss auf die Studiendauer hat. Um plausible Daten zu erhalten, kann der Verlauf der Variablen Berufstätigkeit mittels einer retrospektiven Befragung nur approximativ erhoben werden - und zwar in durchschnittlichen Wochenstunden je Semester, Studien- oder Kalenderjahr. Studierende, bei denen häufig die Arbeitsintensität wechselt, werden sich nur ungenau an ihr Berufsausmaß je Monat zurückerinnern; siehe Abschnitt 10.2. Zudem werden die Befragungen mit der Einbeziehung von zeitveränderlichen Variablen zunehmend komplexer.

\section{Notwendigkeit von spezifischen Analyse-Methoden}

Es sind vor allem zwei Gründe, warum ereignisorientierte Daten (meist) nur mit großem Informationsverlust mit konventionellen statistischen Modellen behandelt werden können: zensierte Daten und zeitabhängige Variablen. Ereignisorientierte Studien enthalten - wie bereits erwähnt - häufig Daten, für die im Beobachtungszeitraum das interessierende Ereignis nicht eingetroffen ist; der Ereigniszeitpunkt liegt daher in der Zukunft und ist zum Endzeitpunkt der Beobachtungsperiode nicht bekannt (rechts-zensierte Daten). Für diese Daten kann daher keine Zeitdauer bis zum Eintreffen des Ereignisses angegeben werden, jedoch kennt man mit dem Ende des Beobachtungszeitraums eine untere Schranke für die zu untersuchende Zeitdauer. Die Nicht-Berücksichtigung dieser Information durch Weglassen dieser Daten kann - vor allem bei einem hohen Anteil solcher Fälle - zu stark verzerrten Schätzern - etwa der erwarteten Dauer - führen.

Mit Hilfe von Logit- und Probit-Modellen (siehe Aldrich und Nelson (1987) für einen Überblick) kann etwa die Information, dass für Untersuchungseinheiten das Ereignis bis zu einem gewissen Zeitpunkt nicht eingetroffen ist, einbezogen werden. Die Ausprägungen der abhängigen dichotomen Variablen eines Modells für den Studienerfolg können beispielsweise sein: "abgeschlossen" (bis zum Ende der Beobachtungsperiode) oder "noch im Studium". In diesem Modell geht jedoch die Information der exakten Studiendauer der Absolventen verloren, die Schätzungen hängen von dem gewählten Ende des Beobachtungszeitraums ab. In linearen Regressionsmodellen (siehe Draper und Smith (1981) für einen Überblick) mit der Dauer bis zum erfolgreichen Studienabschluss als abhängige Variable können wiederum rechts-zensierte Daten nicht einbezogen 
werden. In beiden Modellen werden auch nicht die Daten von Studienabbrechern berücksichtigt.

Im Rahmen eines ereignisorientierten Versuchsplans können Variablen, die sich mit der Zeit ändern, beobachtet werden. Veränderungen der Werte dieser Variablen im Laufe des dynamischen Prozesses können die Dauer bis zum Eintreffen des interessierenden Ereignisses stark beeinflussen. Beispielsweise ist zu vermuten, dass der Beginn einer Berufstätigkeit im Laufe des Studiums die Chancen auf einen schnellen Studienabschluss verringert. Ebenso können zeitvariierende Effekte von Variablen analysiert werden. Es ist etwa zu vermuten, dass manche Variablen zu Beginn des Studiums einen Einfluss auf das Studienabbruch-Risiko haben, der mit der Zeit nachlässt. Konventionelle Methoden bieten nicht so flexible Möglichkeiten, zeitabhängige Variablen in das Modell zu integrieren und zeitliche Änderungen von Effekten zu analysieren.

Im Laufe der vorliegenden Arbeit wird erläutert, in welcher Form Ereignisdatenmodelle die Implementierung von zensierten Daten und zeitabhängigen Variablen erlauben; siehe Abschnitte 3.2, 5.2 und 6.3.

\section{Klassifikation der Verfahren}

Im nächsten Kapitel werden die Grundkonzepte der Ereignisdatenanalyse und in den darauffolgenden Kapiteln die gängigen Methoden zur Analyse von Ereignisdaten erläutert. Dabei unterscheiden wir zwischen

- nicht-parametrischen, semiparametrischen und parametrischen Modellen; nicht-parametrische Methoden beschreiben die Verteilung der Zeitdauer bis zu einem Ereignis, semiparametrische und parametrische Modelle wollen vor allem den Effekt von potentiellen Einflussfaktoren ermitteln.

- kontinuierlichen und diskreten Modellen; können etwa die Ereignisse zu beliebigen Zeitpunkten auftreten und sind die Ereigniszeitpunkte genau genug gemessen, sodass die Anzahl gleicher Beobachtungswerte nicht zu groß ist, werden kontinuierliche Modelle verwendet, ansonsten diskrete Modelle. Wird die Dauer bis zum erfolgreichen Studienende in Monaten angegeben, so eignet sich ein kontinuierliches Ereignisdatenmodell, bei Messung der Studiendauer in Semestern sind diskrete Modelle zu verwenden.

- Modellen mit einem Anfangszustand und einem Endzustand und den sogenannten Mehr-Zustands- und Mehr-Epsioden-Modellen; bei Mehr-ZustandsModellen wird zwischen verschiedenen Ereignisarten unterschieden, die die Periode in einem Anfangszustand beenden - beispielsweise beendet sowohl 
der Studienabschluss als auch der Studienabbruch das Studium - und bei Mehr-Episoden-Modellen durchläuft die Untersuchungseinheit mehrere Perioden, etwa Studienphasen bzw. -abschnitte. 


\section{Kapitel 3}

\section{Grundkonzepte}

Die Grundbegriffe, die in diesem Kapitel erläutert werden, und die in den darauffolgenden Kapiteln behandelten Methoden werden für kontinuierliche Modelle mit einem Anfangszustand und einem Endzustand definiert. Die Erweiterungen der Begriffe und Methoden auf Mehr-Zustands- und Mehr-EpisodenModelle und auf diskrete Modelle werden in den beiden entsprechenden Kapiteln 7 und 8 erläutert.

\subsection{Hazardrate, Überlebens- und Dichtefunk- tion}

Es sei $T$ die Zeitdauer bis zum Eintritt eines bestimmten Ereignisses; sie ist eine nicht-negative stetige Zufallsvariable und $f(t)$ und $F(t)$ die Dichte- und Verteilungsfunktion von $T$. Dann wird mit

$$
S(t)=P(T>t)=1-F(t)
$$

die Überlebensfunktion (survivor- oder survival-Funktion) bezeichnet. Sie gibt die Wahrscheinlichkeit dafür an, dass ein Individuum den Zeitpunkt $t$ "erlebt" hat, das heißt, dass bis zu diesem Zeitpunkt noch kein Ereignis eingetreten ist. Für die Überlebensfunktion $S(t)$ gilt $S(0)=1$, und ihr Verlauf ist monoton fallend.

Eine zentrale Größe bei der Analyse von Ereignisdaten ist die Hazardfunktion bzw. Hazardrate. Sie gibt das augenblickliche Risiko zum Zeitpunkt $T=t$ an, dass das betrachtete Ereignis eintrifft - unter der Voraussetzung, dass das Ereignis nicht vor $t$ eingetreten ist. Daher wird sie auch als Risikofunktion 
bezeichnet. Sie ist definiert als

$$
h(t)=\lim _{\Delta t \rightarrow 0} \frac{1}{\Delta t} P(t \leq T<t+\Delta t \mid T \geq t) .
$$

Die Hazardrate kann als Grenzwert der bedingten Wahrscheinlichkeit aufgefasst werden, dass die Episode im Intervall $[t, t+\Delta t)$ zu Ende geht - unter der Voraussetzung, dass das interessierende Ereignis nicht schon vor Beginn des Intervalls eingetroffen ist. Die Hazardrate kann nicht als bedingte Wahrscheinlichkeit interpretiert werden; sie ist nicht-negativ, kann aber Werte größer als Eins annehmen. Das Produkt $h(t) \Delta t$ kann jedoch als Approximation der bedingten Wahrscheinlichkeit $P(t \leq T<t+\Delta t \mid T \geq t)$ aufgefasst werden.

Mit Hilfe von Hazardraten ist es möglich zu überprüfen, wie sich das Ereignisrisiko (oder im positiven Sinne die Chance auf ein Ereignis) mit der Zeit ändert. In vielen Anwendungsfällen besitzt man ein a-priori Wissen über die Gestalt der Hazardrate. Beispielsweise ist zu vermuten, dass das StudienabbruchRisiko (das durch die Hazardrate dargestellt werden kann) einen badewannenförmigen Verlauf hat. Das Abbruchrisiko ist zu Beginn des Studiums hoch, fällt dann $a b$ und bleibt über einen bestimmten Zeitraum konstant, bis es wieder mit zunehmender Studiendauer leicht ansteigt. Das Sterberisiko bei den Menschen und das Ausfallsrisiko vieler technischer Geräte folgen einem ähnlichen Verlauf. Die Hazardrate für den Abschluss eines Studiums (Studienabschlussrate) an der Wirtschaftsuniversität steigt (vermutlich) nach dem achten Semester (Mindeststudiendauer) stark an, um später wieder leicht zu fallen; siehe Kapitel 12.

Das Integral

$$
H(t)=\int_{0}^{t} h(u) d u
$$

wird als kumulative Hazardrate bezeichnet.

Aus diesen Definitionen lassen sich folgende Beziehungen zwischen den drei Größen herleiten:

$$
\begin{aligned}
h(t) & =\frac{f(t)}{S(t)} \\
S(t) & =\exp \left(-\int_{0}^{t} h(u) d u\right)=\exp [-H(t)] \\
f(t) & =h(t) S(t)=h(t) \exp \left(-\int_{0}^{t} h(u) d u\right)
\end{aligned}
$$

Diese Beziehungen lassen erkennen, dass Dichte-, Überlebens- und Hazardfunktion äquivalente Möglichkeiten zur Beschreibung des Prozessverlaufs sind. 
Ist eine der drei Funktionen festgelegt, sind die beiden anderen Funktion eindeutig ableitbar. Eine Herleitung dieser Beziehungen und ein systematischer Überblick über die Beziehungen zwischen den drei Funktionen sind beispielsweise in Wangler (1997) zu finden.

Die erwartete Dauer, $E(T)$, die erwartete Restdauer zu einem bestimmten Zeitpunkt $t_{1}, E\left(T-t_{1} \mid T>t_{1}\right)$, und die $\operatorname{Varianz}, \operatorname{Var}(T)$, lassen sich wie folgt berechnen:

$$
\begin{aligned}
E(T) & =\int_{0}^{\infty} t f(t) d t=\int_{0}^{\infty} S(t) d t \\
E\left(T-t_{1} \mid T>t_{1}\right) & =\frac{\int_{t_{1}}^{\infty}\left(t-t_{1}\right) f(t) d t}{S\left(t_{1}\right)}=\frac{\int_{t_{1}}^{\infty} S(t) d t}{S\left(t_{1}\right)} \\
\operatorname{Var}(T) & =2 \int_{0}^{\infty} t S(t) d t-\left[\int_{0}^{\infty} S(t) d t\right]^{2}
\end{aligned}
$$

Das p-Quantil $t_{p}$ erhält man, indem man die Gleichung $S\left(t_{p}\right)=1-p$ löst, also $t_{p}=S^{-1}(1-p)$.

Wahrscheinlichkeitsverteilungen, die typischerweise zur Beschreibung von Verläufen verwendet werden, behandelt - unter Einbeziehung von Kovariablen ausführlich das Kapitel 5.1.

\subsection{Zensieren und Abschneiden}

Bei der Analyse von Zeitdauern bis zu einem betrachteten Ereignis ist man in der Regel mit unvollständigen Zeitdauern oder mit einer eingeschränkten (selektiven) Grundgesamtheit, die ein bestimmtes Ereignis schon erlebt hat, konfrontiert. Besonders häufig treten bei Daten, die nach einem ereignisorientierten Versuchsplan erhoben werden, - wie im Abschnitt 2.2 erwähnt - Fälle auf, die durch das Ende des Beobachtungszeitraums zensiert werden.

Zensierung aufgrund des Stichtags (Ende des Beobachtungszeitraums) der Studie fällt unter den Typ Rechts-Zensieren, für den folgende Fälle unterschieden werden können:

1. Typ I - Zensierung tritt auf, falls die Studie nach einer fixen Zeitperiode beendet wird, und sich noch Elemente in der Risikomenge befinden. Bei Analysen der Studiendauer von Studieneingangs-Kohorten etwa gehören jene Studierenden, die zum festgelegten Studienende noch studieren, zu diesem Typ. 
2. Typ II - Zensierung tritt auf, falls die Studie nach einer bestimmten, fix vorgegebenen Anzahl $r<n$ von eingetroffenen Ereignissen beendet wird.

In beiden Fällen ist von den Elementen, die zu Studienende noch in der Risikomenge sind, bekannt, dass für sie das interessierende Ereignis bis zum Studienende nicht eingetroffen ist.

3. Bei der zufälligen Zensierung werden die Zensierungszeiten im Unterschied zu den eben angeführten Fällen als Realisierungen von Zufallsvariablen gesehen. Die Zensierung erfolgt während der Risikoperiode durch Eintreffen eines vom betrachteten Ereignis verschiedenen, vom Zufall abhängigen Ereignisses. Der Tod durch Unfall ist ein typisches Beispiel für eine zufällige Zensierung eines Individuums der Risikomenge.

Die Einbeziehung von Typ I und II zensierten Fällen in Ereignisdatenmodelle ist problemlos; für die Einbeziehung zufällig rechts-zensierter Daten verlangen jedoch alle Standardmethoden der Ereignisdatenanalyse, dass die Ereigniszeiten $T_{i}$ und die Zensierungszeiten $C_{i}$ - unter Berücksichtigung der einbezogenen Kovariablen bedingt - unabhängig sind; siehe Abschnitt 5.2. Kalbfleisch und Prentice (1980) beschreiben diese Bedingung, die ausführlich in Lagakos (1979) diskutiert wird, wie folgt: "Essentially we require that, conditionally on the values of any explanatory variables, the items withdrawn from risk at time $t$ should be "representative" of the items at risk. In particular, items cannot be censored because they appear to be at unusually high or low risk of failure".

In vielen Anwendungsfällen ist jedoch zu vermuten, dass diese Bedingung nicht erfüllt ist. Die beiden folgenden Beispiele sollen diese wichtige Voraussetzung näher bringen:

Unabhängiges Zensieren: Analysiert man beispielsweise die Zeitdauer von der Diagnose von Krebs bis zum Tod und stirbt ein in der Studie befindliches Individuum bei einem Autounfall, so spricht man von unabhängiger Zensierung, da der Tod durch Autounfall unabhängig vom Tod durch Krebs ist.

Bedingt (un)abhängiges Zensieren: Bei der Analyse von Studiendauern liegt beispielsweise die Vermutung nahe, dass jene Studierenden, die das Studium früher abbrechen, eher solche Studierenden sind, die für das Studium länger gebraucht hätten. Unter der Annahme, dass der Effekt des Studienabbruchs auf den Studienabschluss durch die in das Modell einbezogenen Kovariablen nicht vollständig erklärt wird, spricht man von einer (residualen) abhängigen Zensierung. Kann man jedoch davon ausgehen, dass die Abhängigkeit der Zensierungs- und Ereigniszeiten vollständig durch die 
einbezogenen Kovariablen erklärt wird, spricht man von einer bedingt unabhängigen Zensierung.

Sowohl das Analysieren von Studiendauern als auch von "Lebensdauern" von Geräten zeigt, dass (bedingt) (un)abhängiges Zensieren ein wichtiges Thema im Fall von Mehr-Zustands-Modellen ist. Im ersten Beispiel kann das Studium durch Abbruch oder Abschluss beendet werden und im zweiten Beispiel können Geräte aus verschiedenen Gründen ausfallen, die voneinander abhängig sein können oder nicht. Daher wird diese Thematik auch im Kapitel 7 ausführlich diskutiert. Abbildung 3.1 gibt ein Beispiel für Typ I und zufällige Zensierungszeiten.

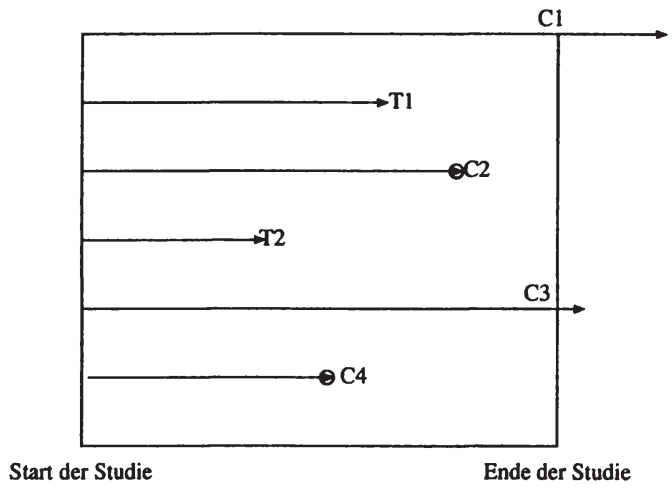

Abbildung 3.1: Beispiel von Typ I zensierten $\left(C_{1}\right.$ und $\left.C_{3}\right)$, zufällig zensierten Zeiten $\left(C_{2}\right.$ und $\left.C_{4}\right)$ und Ereigniszeiten $T_{i}$

Unter links-zensierten Daten werden jene Fälle verstanden, bei denen nur bekannt ist, dass das betrachtete Ereignis irgendwann vor einem bekannten Zeitpunkt eingetreten ist. Beispiele für links-zensierte Zeitdauern sind:

- In einer Analyse der Studiendauer von WU-Studierenden seit Beginn ihres ersten (nicht notwendigerweise an der Wirtschaftsuniversität) inskribierten Studiums anhand der in den administrativen Datenbanken der Wirtschaftsuniversität verfügbaren Daten ist von Wechselstudenten nur bekannt, dass sie vor ihrem WU-Studienbeginn schon an einer anderen Universität inskribiert waren.

- In einer Studie wurden Schüler über 14 nach ihrem Alter beim ersten Drogenkonsum gefragt. Einige antworteten, dass sie schon Drogen genommen haben, allerdings das Alter des ersten Drogenkonsums nicht mehr genau 
wissen. Dann ist lediglich bekannt, dass der Zeitpunkt des ersten Drogenkonsums vor der Befragung war; siehe Klein und Moeschberger (1997).

- In einer Studie der Lebensdauer seit dem Infizieren mit dem HIV-Virus ist (meist) nur der Diagnosezeitpunkt bekannt, jedoch weiß man vom Zeitpunkt des Infizierens nur, dass er vor dem Diagnosezeitpunkt liegt.

Eine Untersuchungseinheit heißt intervall-zensiert, falls lediglich bekannt ist, dass das interessierende Ereignis im Intervall $(a, b)$ eingetroffen ist. LinksZensierung und Rechts-Zensierung können als Spezialfälle gesehen werden, in$\operatorname{dem} a=0$ bzw. $b=\infty$ gesetzt wird. Bei der Analyse des Studienabruch-Risikos ist mit dem im System eingetragenen Abbruchdatum nur eine obere Grenze und mit dem Datum der letzten erbrachten Leistung eine untere Grenze des eigentlichen Datums des Studienabbruchs bekannt; die Daten sind intervallzensiert.

Beim Abschneiden (engl. truncation) werden nur jene Untersuchungseinheiten in die Studie miteinbezogen (selektiert), bei denen ein bestimmtes qualifizierendes Ereignis eingetroffen ist.

Im Falle des Links-Abschneidens werden etwa nur jene Untersuchungseinheiten in die Studie aufgenommen, die einen bestimmten Zeitpunkt oder ein bestimmtes Ereignis vor dem interessierenden Ereignis bis zum Ende des Beobachtungszeitraums erlebt haben. Von diesen Individuen ist dann bekannt, dass sie in der Zeitdauer $V$ vom Studienbeginn bis zum qualifizierenden Ereignis bzw. fixen Zeitpunkt zwar dem Risiko eines Ereignisses ausgesetzt waren, das Ereignis jedoch nicht eingetroffen ist; somit gilt $T>V$.

Die folgenden Beispiele sollen den Begriff Links-Abschneiden näher bringen:

- In einer Studie des Studienabbruch-Risikos von Studierenden des zweiten Studienabschnitts werden nur jene Studierenden einbezogen, die bis zum Ende der Beobachtungsperiode den ersten Abschnitt beendet haben. Jene Studierenden, die das Studium vor Beendigung des ersten Abschnitts abgebrochen haben oder bei Ende der Beobachtungsperiode den ersten Abschnitt noch nicht beendet haben, werden nicht berücksichtigt. Von den in die Studie aufgenommenen Studierenden ist jedoch bekannt, dass sie während des ersten Abschnittes nicht abgebrochen haben.

- Aus einer administrativen Datenbank war bekannt, dass es am 1. Juli 1973 in einem bestimmten Teil Dänemarks 1499 Insulin-abhängige Diabetiker gab. In einer Studie der Lebensdauer seit Diagnose der Krankheit wurden diese Diabetiker bis zum Stichtag 1. Jänner 1982 verfolgt. Dem Design der Studie 
entsprechend wurden also nur jene Diabetiker in die Studie aufgenommen, die am 1. Juli 1973 noch am Leben und Insulin-abhängig waren; es handelt sich daher um links-abgeschnittene Fälle; siehe Andersen et al. (1993).

- In einer Firma wird die Hazardrate für einen Jobwechsel in Abhängigkeit von der Dauer des Arbeitsverhältnisses in dieser Firma (und von ergänzenden Kovariablen) modelliert. Dazu werden zu Beginn der Studie alle Mitarbeiter befragt, wie lange sie schon für diese Firma arbeiten. Danach werden sie entweder bis zum Arbeitsplatzwechsel oder zum Ende der Beobachtungsperiode prospektiv beobachtet. Auch in diesem Beispiel werden nur Mitarbeiter in die Studie miteinbezogen, die nicht schon vor Beginn der Studie die Firma verlassen haben.

- Interessieren in einer Studie der Lebensdauer seit dem Diagnosezeitpunkt von Leukämie nur jene Patienten, bei denen eine Knochenmarkstransplantation (= Zwischenereignis) durchgeführt worden ist, so sind dies auch linksabgeschnittene Fälle; für sie ist die Lebensdauer seit Diagnose größer als die Dauer bis zur Transplantation.

Beim Rechts-Abschneiden werden nur Fälle in die Studie aufgenommen, die das interessierende Ereignis bereits erlebt haben. Die Statistik Österreich (ehemals Österreichisches Statistisches Zentralamt) erhebt bei allen Universitätsabsolventen einige Daten zum Studium und zur Person. Untersuchungen der Studiendauer auf Basis dieser Absolventendatenbanken basieren auf rechtsabgeschnittenen Daten.

Ein weiteres Beispiel ist etwa die Untersuchung der Zeitdauer vom Infizieren bis zum Ausbruch von AIDS, in der nur Daten von jenen Patienten verfügbar sind, bei denen AIDS bereits ausgebrochen ist. Dies tritt häufig in administrativen Datenbanken auf, wo ein Fall erst nach dem Ereigniszeitpunkt mit dem Datum des Infizierens und einigen zusätzlichen Kovariablen retrospektiv registriert wird; siehe Bilker und Wang (1996).

Im Zuge dieser Arbeit - bei der Beschreibung der statistischen Verfahren der Ereignisanalyse und bei der Analyse der Studiendauer und des StudienabbruchRisikos - wird vor allem auf Typ I und zufällig rechts-zensierte Daten eingegangen, aber auch links-abgeschnittenen Daten werden behandelt. Das Problem des (bedingt) (un)abhängigen zufälligen Zensierungsmechanismus wird ebenfalls diskutiert. Links-zensierte und rechts-abgeschnittene Daten und ihre Modellierung sind nicht Thema dieser Arbeit.

Ausführlich wird das Thema "Zensieren und Abschneiden" - mit illustrativen Beispielen aus der Medizin - beispielsweise in Andersen et al. (1993) und Klein und Moeschberger (1997) behandelt. 


\section{Kapitel 4}

\section{Nicht-parametrische Verfahren}

Nicht-parametrische Verfahren zur Schätzung der Überlebensfunktion dienen der vorbereitenden Analyse der Daten. Sie geben einen Einblick in den Prozessverlauf, ermöglichen Subgruppenvergleiche, die erste Aufschlüsse über die Wichtigkeit bestimmter Variablen zulassen. Daneben eignen sie sich zur graphischen Überprüfung der getroffenen Verteilungsannahmen für die Zeitdauer $T$; siehe Abschnitt 6.5.

Dieses Kapitel gibt einen kurzen Überblick über die Kaplan-Meier-, NelsonAalen- und Sterbetafel-Methode unter Berücksichtigung von (unabhängigen) rechts-zensierten Daten. Im folgenden wird davon ausgegangen, dass es sich bei den Untersuchungseinheiten um Individuen handelt.

\subsection{Kaplan-Meier- und Nelson-Aalen-Methode}

Das am häufigsten verwendete Verfahren zur nicht-parametrischen Schätzung der Überlebensfunktion $S(t)$ ist die Kaplan-Meier-Methode; siehe Kaplan und Meier (1958). Enthält der Datensatz keine zensierten Fälle, dann entspricht der Kaplan-Meier-Schätzer zum Zeitpunkt $t, \hat{S}(t)$, dem Stichprobenanteil der Beobachtungen mit Ereigniszeiten größer als $t$, d.h $\hat{S}(t)=1-\hat{F}(t)$, wobei $\hat{F}(t)$ die empirische Verteilungsfunktion ist.

Zur Konstruktion des Kaplan-Meier-Schätzers unter Berücksichtigung von (unabhängigen) rechts-zensierten Daten nehmen wir an, dass es $k \leq n$ verschiedene, geordnete Ereigniszeiten $t_{(1)}<t_{(2)}<\ldots<t_{(k)}$ gibt, und $d_{i}$ die Anzahl der Ereignisse zum Zeitpunkt $t_{(i)}$ ist. Unmittelbar vor jedem Zeitpunkt $t_{(i)} \operatorname{sind} n_{i}$ Individuen noch dem Risiko ausgesetzt, dass das Ereignis zum Zeitpunkt $t_{(i)}$ 
eintritt. Das sind also jene Fälle, die bis unmittelbar vor $t_{(i)}$ weder ein Ereignis erlebt haben noch zensiert worden sind. Diese Individuen werden auch als Risikomenge zum Zeitpunkt $t_{(i)}, R\left(t_{(i)}\right)$, bezeichnet. Falls es Fälle gibt, die genau zum Zeitpunkt $t_{(i)}$ zensiert werden, zählt man sie noch zur Risikomenge $R\left(t_{(i)}\right)$ dazu. Der Kaplan-Meier-Schätzer wird für Zeiten bis zur letzten beobachteten Ereignis- oder Zensierungszeit $t_{\max }$ definiert als

$$
\hat{S}(t)= \begin{cases}1 & \text { für } t<t_{(1)} \\ \prod_{t_{(i)} \leq t}\left(1-\frac{d_{i}}{n_{i}}\right) & \text { für } t_{(1)} \leq t \leq t_{\text {max }}\end{cases}
$$

Gilt $t_{\max }=t_{(k)}$, die maximale beobachtete Zeit somit eine Ereigniszeit ist, dann ist $\hat{S}(t)=0$ für $t>t_{\text {max }}$. Gibt es jedoch Zensierungszeiten, die größer als die maximale Ereigniszeit sind, dann ist der Kaplan-Meier-Schätzer für Zeiten größer als die letzte beobachtete Zensierungszeit $\left(t>t_{\max }\right)$ nicht definiert. Zur Schätzung von $S(t)$ in diesem Bereich finden sich in der Literatur verschiedene nicht-parametrische Ansätze; siehe Klein und Moeschberger (1997) für einen kurzen Überblick. Der Ausdruck $\frac{d_{i}}{n_{i}}$ ist ein Schätzer für die bedingte Wahrscheinlichkeit, dass für ein Individuum, das den Zeitpunkt $t_{(i-1)}$ erlebt hat, das Ereignis zum Zeitpunkt $t_{(i)}$ eintrifft.

Der Kaplan-Meier-Schätzer ist eine Treppenfunktion mit Sprungstellen an den beobachteten Ereigniszeiten. Aus (4.1) ist zu erkennen, dass die Gestalt des Kaplan-Meier-Schätzers $\hat{S}(t)$ nicht nur von den beobachteten Ereigniszeiten sondern auch von den Zensierungszeiten abhängt.

Eine Schätzung der Varianz von $\hat{S}(t)$ erhält man beispielsweise mit Hilfe der Formel von Greenwood (1926):

$$
\widehat{\operatorname{Var}}[\hat{S}(t)]=\hat{S}(t)^{2} \sum_{t_{(i)} \leq t} \frac{d_{i}}{n_{i}\left(n_{i}-d_{i}\right)} .
$$

Das $p$-te Perzentil der Dauer bis zum Ereignis wird geschätzt mit

$$
\hat{t}_{p}=\min \{t: \hat{S}(t) \leq(p / 100)\},
$$

und ein Schätzer für die erwartete Dauer bis zum Ereignis ist

$$
\hat{\mu}(t)=\int_{0}^{\infty} \hat{S}(t) d t
$$

Dieser Schätzer ist nur geeignet, wenn die letzte Beobachtung nicht zensiert ist, da sonst der Kaplan-Meier-Schätzer für Zeiten größer als die maximale beobachtete Zensierungszeit $t_{\max }$ nicht definiert ist. Ist die letzte Beobachtung zensiert, wird die erwartete Dauer bis zum Ereignis mit

$$
\hat{\mu}\left(t^{*}\right)=\int_{0}^{t^{*}} \hat{S}(t) d t
$$


geschätzt, wobei für $t^{*}$ meist die maximale beobachtete Ereigniszeit oder die maximale Zensierungszeit eingesetzt wird. Im ersten Fall erhält man (unabhängig davon, ob die letzte Beobachtung zensiert ist oder nicht)

$$
\hat{\mu}\left(t_{(k)}\right)=\sum_{i=1}^{k} \hat{S}\left(t_{(i-1)}\right)\left(t_{(i)}-t_{(i-1)}\right) .
$$

und im zweiten Fall

$$
\hat{\mu}\left(t_{\max }\right)=\hat{\mu}\left(t_{(k)}\right)+\left(1-\delta_{\max }\right) \hat{S}\left(t_{(k)}\right)\left(t_{\max }-t_{(k)}\right),
$$

wobei $t_{\max }$ die letzte Beobachtung und $\delta_{\max }$ der Zensierungsstatus dieser Beobachtung ist:

$$
\delta_{\max }= \begin{cases}1 & \text { falls } t_{\max } \text { die maximale Ereigniszeit ist }\left(=t_{(k)}\right) \\ 0 & \text { falls } t_{\max } \text { rechts-zensiert ist. }\end{cases}
$$

Beide Schätzer sind nach unten verzerrt. Aus diesen Gründen wird in praktischen Analysen der Median als Schätzer der erwarteten Dauer bis zum betrachteten Ereignis bevorzugt.

Hosmer und Lemeshow (1999) sowie Klein und Moeschberger (1997) erläutern detailliert, wie mit Hilfe des Kaplan-Meier-Schätzers punktweise Konfidenzintervalle und Konfidenzbänder für die Überlebensfunktion sowie Punkt- und Intervallschätzer für den Erwartungswert und die Quantile der betrachteten Zeitdauer (bis zum Ereignis) berechnet werden können. Klein und Moeschberger (1997) gehen auch auf die Konstruktion des Kaplan-Meier-Schätzers im Falle von links-abgeschnittenen Daten ein.

Der Kaplan-Meier-Schätzer $\hat{S}(t)$ kann auch zur Schätzung der kumulativen Hazardfunktion $H(t)$ verwendet werden:

$$
\hat{H}(t)=-\ln [\hat{S}(t)]
$$

Mit Hilfe der Theorie der Zählprozesse haben Nelson (1972) und Aalen (1978) einen alternativen Schätzer für die kumulative Hazardfunktion entwickelt, der bis zur maximalen beobachteten Zeit $t_{\max }$ definiert wird als

$$
\begin{cases}0 & \text { für } t<t_{(1)} \\ \tilde{H}(t)=\sum_{t_{(i)} \leq t} \frac{d_{i}}{n_{i}} & \text { für } t_{(1)} \leq t \leq t_{\max }\end{cases}
$$

und sich auch als erste Näherung aus dem Kaplan-Meier-Schätzer ergibt. Somit erhält man als alternativen Schätzer für die Überlebensfunktion

$$
\tilde{S}(t)=\exp [-\tilde{H}(t)]
$$


Im Abschnitt 5.3 wird gezeigt, dass die kumulative Hazardrate und damit sowohl der Kaplan-Meier- als auch der Nelson-Aalen-Schätzer bei der graphischen Überprüfung der Verteilungsannahmen für die Dauer bis zum Ereignis von Bedeutung sind.

Details zur Herleitung des Kaplan-Meier-Schätzers und des Nelson-Aalen-Schätzers mit Hilfe der Theorie der Zählprozesse und Martingale sowie zu ihren statistischen Eigenschaften, etwa dass die beiden Schätzer konsistent, asymptotisch äquivalent und normalverteilt sind, sind in Andersen et al. (1993) nachzulesen.

\subsection{Sterbetafel-Methode}

Falls die Zahl der Beobachtungen groß ist, und es viele verschiedene Ereigniszeiten gibt, wird die tabellarische oder graphische Darstellung der Ergebnisse der Kaplan-Meier-Schätzung unübersichtlich, da für jeden Ereigniszeitpunkt $t_{(i)}$ der Schätzer $\hat{S}\left(t_{(i)}\right)$ ermittelt wird. In diesem Fall ist die Sterbetafel-Methode das geeignetere Verfahren, da es die Ereigniszeiten in Intervallen der gewünschten Länge gruppiert.

Für ein Intervall der Form $\left[a_{i}, a_{i+1}\right)$ ist $n_{i}$ die Anzahl der Individuen, die zum Zeitpunkt $a_{i}$ noch dem Risiko eines Ereignisses ausgesetzt ist, das sind jene Fälle, die bis unmittelbar vor $a_{i}$ kein Ereignis erlebt haben und auch nicht zensiert worden sind. Werden diese Individuen über das Intervall verfolgt, erleben $d_{i}$ Individuen das betrachtete Ereignis, und $c_{i}$ Fälle werden zensiert. Die zensierten Fälle sind daher nicht während der gesamten Zeit des Intervalls dem Risiko des Ereignisses ausgesetzt. Für die Berechnung der Risikomenge $R(t), t \in\left[a_{i}, a_{i+1}\right)$, wird daher (zumeist) angenommen, dass die Zensierungszeiten gleichverteilt über das Intervall sind und die durchschnittliche Risikomenge im Intervall somit $n_{i}-c_{i} / 2$ ist. Die bedingte Wahrscheinlichkeit, das $i$-te Intervall zu überleben, erhält man zu

$$
1-\frac{d_{i}}{n_{i}-\left(c_{i} / 2\right)}=1-q_{i}
$$

wobei $q_{i}$ die bedingte Wahrscheinlichkeit für ein Ereignis im $i$-ten Intervall ist, unter der Voraussetzung, dass das Zeitintervall erreicht wurde. Der Schätzer für die Überlebensfunktion ist dann

$$
\hat{S}(t)= \begin{cases}1 & t<a_{1} \\ \prod_{j=1}^{i}\left(1-q_{j}\right) & a_{i} \leq t<a_{i+1}, i>0\end{cases}
$$


Die Kaplan-Meier-Methode ist also ein Spezialfall der Sterbetafel-Methode, bei der die Ereigniszeitpunkte als Intervallgrenzen gewählt werden.

\subsection{Vergleich von Überlebensfunktionen}

Ein weiteres Ziel von ersten deskriptiven Analysen ist der Vergleich der Überlebensfunktionen von Subgruppen, die anhand der Ausprägungen einer oder mehrerer (meist kategorieller) Variablen gebildet worden sind; kontinuierlich skalierte Merkmale müssen gruppiert werden. Bei einer zu großen Anzahl von Untergruppen verliert die Analyse an Übersichtlichkeit, und die Macht der unten angeführten Tests nimmt wegen zu geringer Fallzahlen in den einzelnen Gruppen immer mehr ab. Daher beschränkt sich der in diesem Kapitel beschriebene Vergleich von Überlebensfunktionen auf einige wenige Subgruppen.

Einen ersten Vergleich von Subgruppen ermöglicht die graphische Darstellung der Überlebensfunktionen. Zur formalen Überprüfung der beobachteten Unterschiede sind nicht-parametrische Tests entwickelt worden, die hier für den Vergleich von zwei Gruppen erklärt werden.

Für Individuen aus der Untergruppe 0 bezeichnen wir mit

$n_{0 i} \ldots$ die Anzahl jener Fälle, die unmittelbar vor $t_{(i)}, 1 \leq i \leq k$, noch dem

Risiko eines Ereignisses ausgesetzt sind, und mit

$d_{0 i} \ldots$ die Anzahl der Ereignisse zum Zeitpunkt $t_{(i)}$.

Für die Untergruppe 1 werden die gleichen Größen mit $n_{1 i}$ bzw. $d_{1 i}$ bezeichnet. Dann ist - unter der Nullhypothese, dass die Überlebensfunktionen der beiden Gruppen gleich sind - ein Schätzer für den erwarteten Wert von $d_{1 i}$ gegeben mit

$$
\hat{d}_{1 i}=\frac{n_{1 i} d_{i}}{n_{i}}
$$

wobei $n_{i}=n_{0 i}+n_{1 i}$ und $d_{i}=d_{0 i}+d_{1 i}$. Einen Varianzschätzer für $d_{1 i}$ erhält man auf Basis der hypergeometrischen Verteilung mit

$$
\hat{v}_{1 i}=\frac{n_{1 i} n_{0 i} d_{i}\left(n_{i}-d_{i}\right)}{n_{i}^{2}\left(n_{i}-1\right)} .
$$

Die allgemeine Formulierung der Teststatistik ist

$$
Q=\frac{\left[\sum_{i=1}^{k} w_{i}\left(d_{1 i}-\hat{d}_{1 i}\right)\right]^{2}}{\sum_{i=1}^{k} w_{i}^{2} \hat{v}_{1 i}}
$$


wobei die $w_{i}^{\prime} s$ Gewichte darstellen. Unter der Nullhypothese, dass die beiden Überlebensfunktionen gleich sind, ist die Teststatistik $Q$ (bei unabhängigem Zensierungsmuster in den beiden Untergruppen und großer Anzahl von Ereignissen) stets $\chi^{2}(1)$ verteilt.

Zwei wichtige Spezialfälle sind (i) für $w_{i}=n_{i}$ der verallgemeinerte Wilcoxon Test und (ii) für $w_{i}=1$ der Log-rank-Test bzw. Mantel-Cox-Test.

Ausführlich wird der Vergleich von Überlebensfunktionen beispielsweise in Hosmer und Lemeshow (1999) oder Le (1997) behandelt. In beiden Arbeiten wird auch die Erweiterung der Teststatistik $Q$ auf den Vergleich von mehr als zwei Gruppen diskutiert. 


\section{Kapitel 5}

\section{Parametrische}

\section{Regressionsmodelle}

Neben Ereignis- bzw. Zensierungszeiten wird in der Regel für jedes Individuum eine Reihe von weiteren Variablen erhoben, und ein wichtiges Ziel der statistischen Analyse besteht in der quantitativen Ermittlung des Effektes dieser Variablen auf die Zeitdauer bis zum Eintreffen des Ereignisses. Die Merkmale können quantitativ oder qualitativ sein. Zur Beurteilung des Effektes von potentiellen Einflussgrößen werden Modelle verwendet, die analog zu linearen Regressionsmodellen die (eventuell transformierten) Kovariablen $x_{j}, j=1, \ldots, p$, mit einem Parameter $\beta_{j}$ gewichten und mit $x_{j} \beta_{j}$ in das Modell aufnehmen; kategorielle Variablen werden über eine $(0,1)$-Kodierung der einzelnen Kategorien als Dummy-Variablen in das Modell aufgenommen.

Neben fixen (zeitkonstanten) erklärenden Variablen können auch - im Unterschied zu linearen Regressionsmodellen - zeitveränderliche Variablen in das Modell integriert werden. Bei der Analyse von Studiendauern ist etwa zu vermuten, dass die Berufstätigkeit einen Einfluss auf die Studiendauer hat. Die Berufsintensität bzw. der Berufsstatus (nicht beschäftigt, teilweise beschäftigt, voll beschäftigt) kann sich während der Studienzeit ändern und muss daher realitätsgerecht als zeitveränderliche Variable in das Modell aufgenommen werden.

In diesem Kapitel wird die Analyse der Effekte potentieller Einflussgrößen mit Hilfe von parametrischen Regressionsmodellen erläutert. Das nächste Kapitel behandelt semiparametrische Regressionsmodelle; es werden die Unterschiede zu parametrischen Regressionsmodellen herausgearbeitet und insbesondere wird auf die Einbeziehung von zeitveränderlichen Variablen eingegangen. 


\subsection{Typische Verteilungsannahmen und Ein- beziehung von Kovariablen}

\section{Log-lineare Modelle}

Eine häufig verwendete Klasse von Modellen zur Analyse der Effekte potentieller Einflussfaktoren auf die Dauer bis zum Eintreffen des interessierenden Ereignisses sind Regressionsmodelle, die einen linearen Zusammenhang zwischen den Kovariablen und der logarithmierten Dauer bis zum Ereignis annehmen. Diese log-linearen Modelle, die im englischen Sprachraum als accelerated failure time models bezeichnet werden, sind wie folgt definiert:

$$
\begin{aligned}
\ln T & =\beta_{0}+x_{1} \beta_{1}+\ldots+x_{p} \beta_{p}+\sigma \epsilon=\beta_{0}+\mathbf{x}^{\prime} \boldsymbol{\beta}+\sigma \epsilon \\
T & =\exp \left(\beta_{0}+\mathbf{x}^{\prime} \boldsymbol{\beta}+\sigma \epsilon\right)
\end{aligned}
$$

mit

$$
\begin{array}{rll}
T & \ldots & \text { zufällige Episodendauer } \\
\mathbf{x}=\left(x_{1}, \ldots, x_{p}\right)^{\prime} & \ldots & \text { Werte von } p \text { (zeitunabhängigen) Kovariablen } \\
\epsilon & \ldots & \text { zufälliger Störterm mit einer von } \mathbf{x} \text { unabhängigen } \\
& & \text { Verteilung } \\
\sigma & \ldots & \text { Skalenparameter } \\
\beta_{0} & \ldots & \text { Interzept } \\
\boldsymbol{\beta}=\left(\beta_{1}, \ldots, \beta_{p}\right)^{\prime} & \ldots & \text { Regressionskoeffizienten }
\end{array}
$$

Zeitabhängige Kovariablen und ihre Einbeziehung in semiparametrische und parametrische Regressionsmodelle werden im Abschnitt 6.3 behandelt.

Zur Interpretation des Modells (5.1) betrachten wir das (Basis-)Modell, in dem alle Kovariablen den Wert Null annehmen:

$$
T_{0}=\exp \left(\beta_{0}+\sigma \epsilon\right)
$$

Die Wahrscheinlichkeitsverteilung und Überlebensfunktion von $T_{0}$ sind durch die Wahrscheinlichkeitsfunktion von $\epsilon$ bestimmt. Hat die Zufallsgröße $T_{0}$ die (Basis-) Überlebensfunktion $S_{0}(t)$, dann erhalten wir

$$
\begin{aligned}
S(t \mid \mathbf{x}) & =P(T>t \mid \mathbf{x})=P(\ln T>\ln t \mid \mathbf{x}) \\
& =P\left(\beta_{0}+\sigma \epsilon>\ln t-\mathbf{x}^{\prime} \boldsymbol{\beta} \mid \mathbf{x}\right) \\
& =P\left(\exp \left(\beta_{0}+\sigma \epsilon\right)>t \exp \left(-\mathbf{x}^{\prime} \boldsymbol{\beta}\right) \mid \mathbf{x}\right) \\
& =S_{0}\left[t \exp \left(-\mathbf{x}^{\prime} \boldsymbol{\beta}\right)\right] .
\end{aligned}
$$


Aus dieser Beziehung folgt

$$
t_{0}(p)=\exp \left(-\boldsymbol{x}^{\prime} \boldsymbol{\beta}\right) t_{\mathbf{x}}(p)
$$

Das $p$-Quantil $t_{\mathbf{x}}(p)$ der betrachteten Zeitdauer für Individuen mit Kovariablenvektor $\mathbf{x}$ ist das $\exp \left(\mathbf{x}^{\prime} \boldsymbol{\beta}\right)$-fache des $p$-Quantils $t_{0}(p)$ der Dauer für Individuen mit Kovariablenwerten gleich Null. Zur Interpretation der geschätzten Regressionskoeffizienten können die geschätzten Mediane der zu analysierenden Zeitdauer von Individuen mit unterschiedlichen Ausprägungen der interessierenden Kovariablen (unter Konstanthaltung der anderen einbezogenen Merkmale) verglichen werden. Der Faktor $\exp \left(-\mathbf{x}^{\prime} \boldsymbol{\beta}\right)$ wird Beschleunigungsfaktor (engl. acceleration factor) genannt. Ist dieser Faktor kleiner/größer als 1 , so wirken die Kovariablen für ein Individuum (multiplikativ) verlangsamend/beschleunigend auf die Zeitdauer.

Besonders deutlich wird diese Eigenschaft bei Betrachtung einer binären Variablen. Zur Illustration nehmen wir an, dass einige Studierende zu Beginn ihres Studiums zufällig einer Gruppe zugeordnet werden. An dieser Gruppe sollen in den beiden ersten Semestern einige neue Maßnahmen, die mit dem Ziel der Studienzeitverkürzung eingeführt worden sind, getestet werden. Der Testgruppe wird $x=1$ und den anderen Studienanfängern des Semesters, der Kontrollgruppe, $x=0$ zugeordnet, dann gilt im Falle eines log-linearen Modells

$$
t_{0}(0.5)=\exp (-\beta) t_{1}(0.5) \text {. }
$$

Der Median der Studiendauer der Kontrollgruppe ist damit das $\exp (-\beta)$-fache des Medians der Testgruppe. Ist der geschätzte Regressionskoeffizient $\hat{\beta}$ kleiner als 0 , also $\exp (-\hat{\beta})>1$, dann haben die Maßnahmen eine beschleunigende Wirkung auf die Studiendauer und sind somit als Erfolg zu bezeichnen.

Für die Hazardfunktion erhält man die Beziehung

$$
h(t \mid \mathbf{x})=\exp \left(-\mathbf{x}^{\prime} \boldsymbol{\beta}\right) h_{0}\left[t \exp \left(-\mathbf{x}^{\prime} \boldsymbol{\beta}\right)\right]
$$

wobei $h_{0}$ die Basis-Hazardfunktion ist (für Kovariablenwerte gleich Null), und für die Dichtefunktion

$$
f(t \mid \mathbf{x})=\exp \left(-\mathbf{x}^{\prime} \boldsymbol{\beta}\right) f_{0}\left[t \exp \left(-\mathbf{x}^{\prime} \boldsymbol{\beta}\right)\right]
$$

Die Verteilung von $\epsilon$ bestimmt die entsprechende Verteilung von $T$, und die Regressionsmodelle werden nach der Verteilung von $T$ benannt. Das Exponential-, das Weibull-, das log-logistische-, das log-normale- und das (verallgemeinerte) Gamma-Modell gehören zu dieser Klasse von Regressionsmodellen. 
Beim Exponential-Modell wird vom log-linearen Regressionsansatz mit $\sigma=$ 1 ausgegangen:

$$
\ln T=\beta_{0}+\boldsymbol{x}^{\prime} \boldsymbol{\beta}+\epsilon
$$

Der zufällige Störterm $\epsilon$ folgt der Standard-Extremwert- oder Gumbel-Verteilung:

$$
f(\epsilon)=\exp [\epsilon-\exp (\epsilon)], \quad-\infty<\epsilon<\infty
$$

$T$ besitzt dann bei gegebenem Kovariablenvektor $\mathbf{x}$ die Dichtefunktion

$$
f(t \mid \mathbf{x})=\exp \left(-\beta_{0}-\mathbf{x}^{\prime} \boldsymbol{\beta}\right) \exp \left[-t \exp \left(-\beta_{0}-\mathbf{x}^{\prime} \boldsymbol{\beta}\right)\right]
$$

und die Hazardfunktion

$$
h(t \mid x)=\exp \left(-\beta_{0}-\mathbf{x}^{\prime} \boldsymbol{\beta}\right) .
$$

Im Exponential-Modell ist somit das Risiko für ein Ereignis zum Zeitpunkt $t$ - unter der Voraussetzung, dass das Ereignis bis dahin noch nicht eingetroffen ist - unabhängig von $t$, der Zeit, die das Individuum bereits in der Studie ist.

Im folgenden setzen wir der Einfachheit halber $\exp \left(-\beta_{0}-\mathbf{x}^{\prime} \boldsymbol{\beta}\right)=\lambda(\mathbf{x})$. Für die erwartete Dauer einer Episode und die erwartete Restdauer zum Zeitpunkt $t_{1}$ erhält man bei gegebenem Kovariablenvektor $\mathbf{x}$

$$
E(T \mid \mathbf{x})=E\left(T-t_{1} \mid T>t_{1} ; \mathbf{x}\right)=\frac{1}{\lambda(\mathbf{x})} .
$$

Die zu erwartende Restdauer ist somit für ein Individuum zu jedem Zeitpunkt gleich. Es treten keine Alterungseffekte auf. Trotz dieser restriktiven Eigenschaft, dass der Prozessverlauf von der Zeit unabhängig ist, wurde das Exponential-Modell in der Vergangenheit vor allem wegen seiner mathematischen Einfachheit häufig verwendet. In praktischen Anwendungen eignet sich das realitätsgerechtere stückweise konstante Hazards-Modell, das im Abschnitt 5.4 diskutiert wird, weit mehr. Abbildung 5.1 zeigt die Dichte-, Überlebensund Hazardfunktion der Exponentialverteilung für $\lambda=0.2$.

Beim Weibull-Modell folgt $\epsilon$ der Standard-Extremwert-Verteilung und $\sigma \neq$ 1. Ist $\sigma>1$, dann ist die Hazardfunktion monoton fallend mit der Zeit, für $0.5<\sigma<1$ steigt sie mit abnehmender Rate, für $\sigma=0.5$ mit konstanter Rate und für $0<\sigma<0.5$ steigt sie mit wachsender Rate. Abbildung 5.2 zeigt Hazardfunktionen der Weibull-Verteilung für verschiedene $\sigma$-Werte.

Die Hazardfunktion ist (mit $\delta=1 / \sigma$ )

$$
h(t \mid \mathbf{x})=\delta \lambda(\mathbf{x})^{\delta} t^{\delta-1},
$$




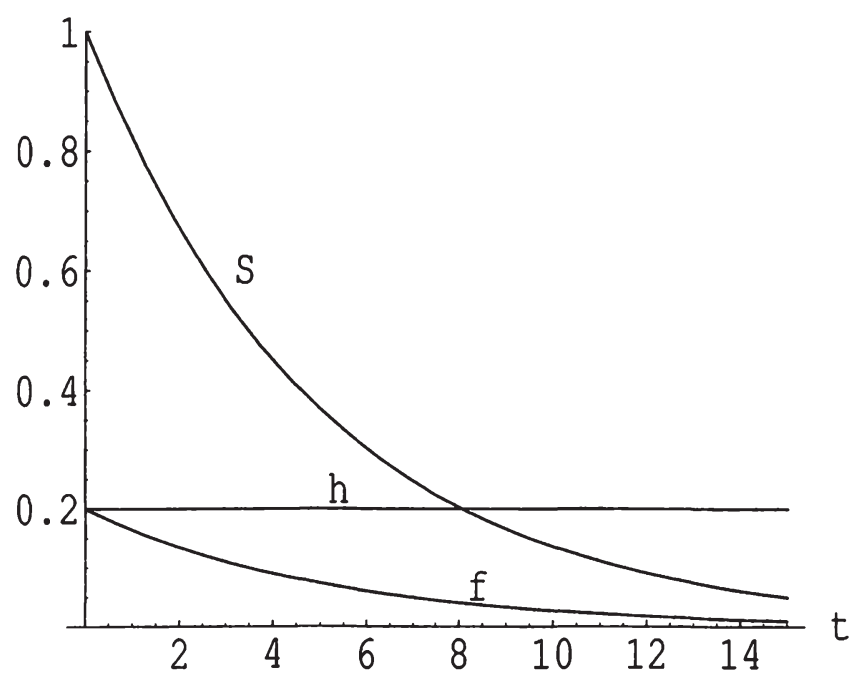

Abbildung 5.1: Dichte- (f), Überlebens- (S) und Hazardfunktion (h) des Exponential-Modells für $\lambda=0.2$

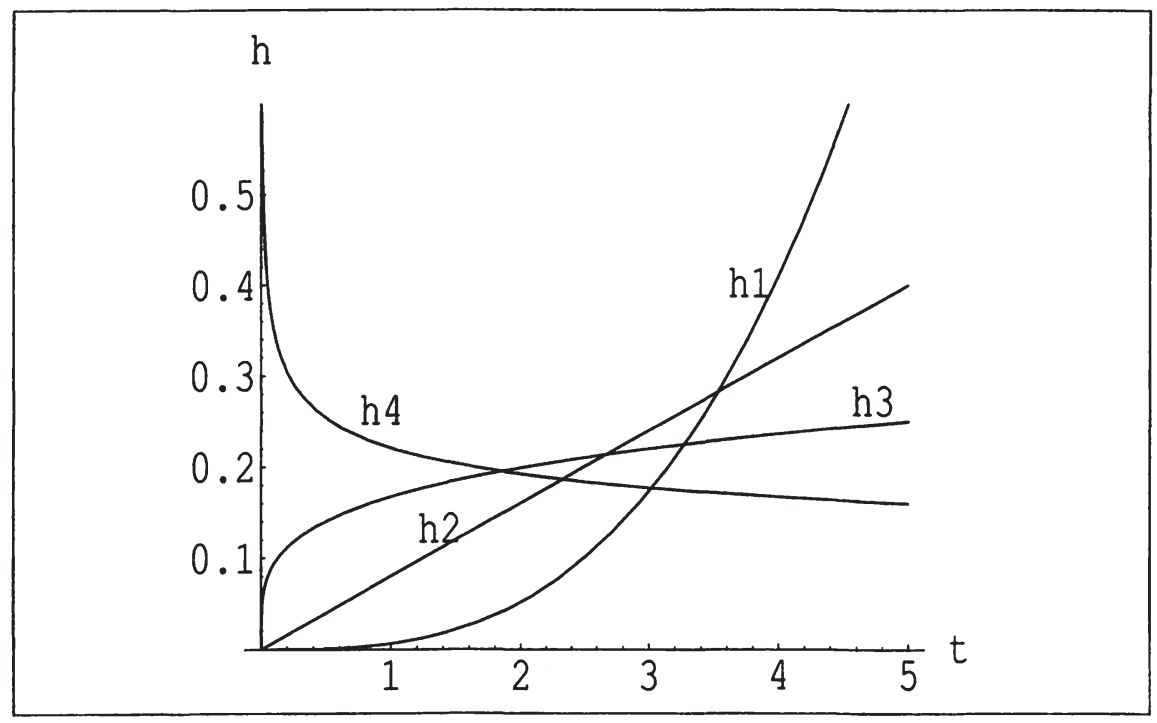

Abbildung 5.2: Hazardraten des Weibull-Modells für $\lambda=0.2$ und $\sigma=0.25\left(h_{1}\right)$, $\sigma=0.5\left(h_{2}\right), \sigma=0.8\left(h_{3}\right), \sigma=1.5\left(h_{4}\right)$ 
die Überlebensfunktion

$$
S(t \mid \mathbf{x})=\exp \left(-[t \lambda(\mathbf{x})]^{\delta}\right)
$$

und $f(t \mid \mathbf{x})=h(t \mid \mathbf{x}) S(t \mid \mathbf{x})$.

Beim log-normalen Modell folgt $\epsilon$ der Standard-Normalverteilung. Das lognormale Modell hat keine monotone Hazardrate; die Hazardrate ist 0 für $t=0$, steigt bis zu einem Maximalwert und konvergiert dann gegen 0 für $t \rightarrow \infty$ (siehe Abbildung 5.3). Die Hazardfunktion kann nicht in geschlossener Form angegeben werden, da sie die Verteilungsfunktion einer standard-normalverteilten Variablen enthält. Dichte- und Überlebensfunktion sind

$$
\begin{aligned}
& f(t \mid \mathbf{x})=\frac{1}{\sqrt{2 \pi}} \frac{1}{t} \exp \left\{-[\log (t)-\mu(\mathbf{x})]^{2} /\left(2 \sigma^{2}\right)\right\} \\
& S(t \mid \mathbf{x})=1-\Phi\left(\frac{\log (t)-\mu(\mathbf{x})}{\sigma}\right),
\end{aligned}
$$

wobei $\Phi$ die Verteilungsfunktion einer standard-normalverteilten Variablen ist und $\mu(\mathbf{x})=-\ln [\lambda(\mathbf{x})]=\beta_{0}+\mathbf{x}^{\prime} \boldsymbol{\beta}$ gilt. Abbildung 5.3 zeigt Hazardfunktionen der log-normalen Verteilung für verschiedene $\sigma$-Werte.

Beim log-logistischen Modell folgt $\epsilon$ der standardisierten logistischen Verteilung mit Dichtefunktion

$$
f(\epsilon)=\frac{\exp \epsilon}{(1+\exp \epsilon)^{2}}, \quad-\infty<\epsilon<\infty .
$$

Für die Hazardfunktion erhält man (mit $\delta=1 / \sigma$ )

$$
h(t \mid \mathbf{x})=\frac{\lambda(\mathbf{x}) \delta(\lambda(\mathbf{x}) t)^{\delta-1}}{1+(\lambda(\mathbf{x}) t)^{\delta}}
$$

und für die Überlebensfunktion

$$
S(t \mid \mathbf{x})=\frac{1}{1+(\lambda(\mathbf{x}) t)^{\delta}} .
$$

Die Hazardfunktion des log-logistischen Modells ist für $\sigma \geq 1$ monoton fallend mit $h(t) \rightarrow \infty$ für $t \rightarrow 0$ bzw. $h(0)=\lambda$ für $\sigma=1$, und $h(t) \rightarrow 0$ für $t \rightarrow \infty$. Für $\sigma<1$ verhält sich die Hazardfunktion ähnlich dem log-normalen Modell. Abbildung 5.4 zeigt Hazardfunktionen der log-logistischen Verteilung für verschiedene $\sigma$-Werte.

Das Exponential-, das Weibull-, das log-normale- und das standardisierte Gamma-Modell (zwei Parameter) sind Spezialfälle des verallgemeinerten Gamma- 


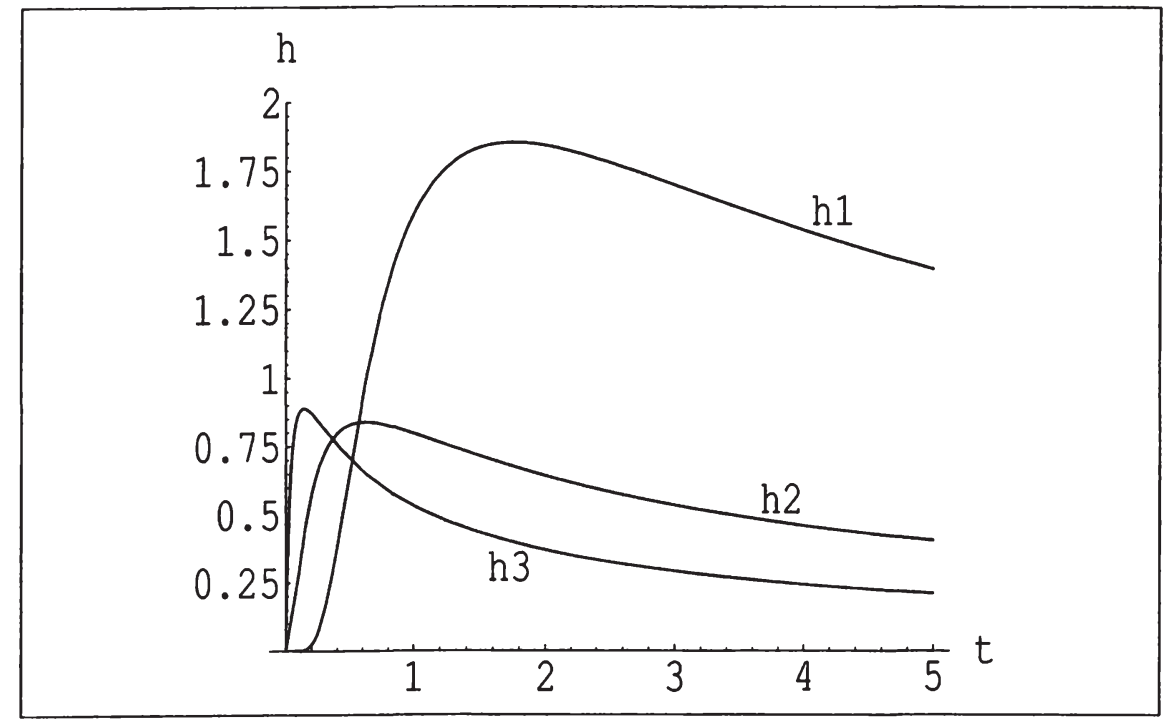

Abbildung 5.3: Hazardraten des log-normalen Modells für $\mu=0$ und $\sigma=$ $0.5\left(h_{1}\right), \sigma=1\left(h_{2}\right), \sigma=1.5\left(h_{3}\right)$

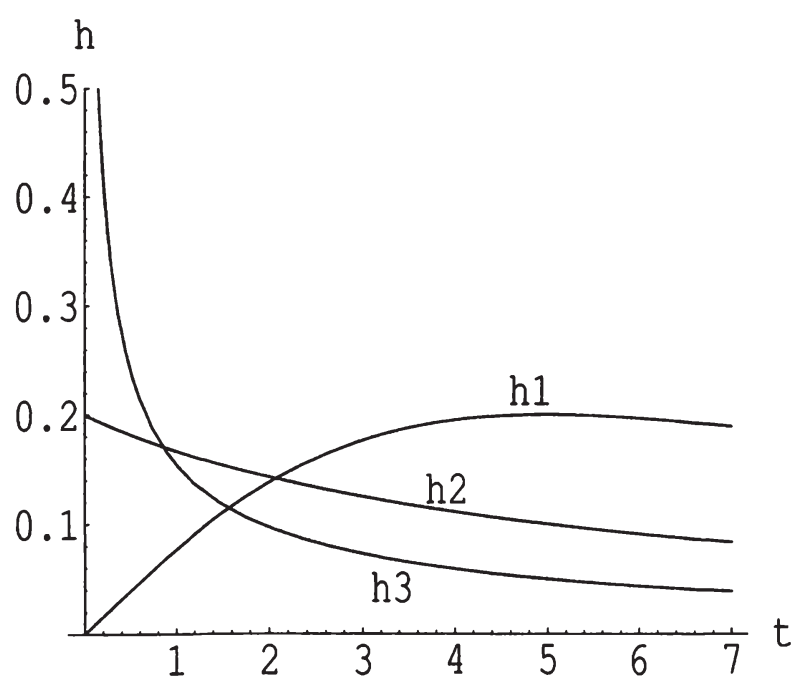

Abbildung 5.4: Hazardraten des log-logistischen Modells für $\lambda=0.2$ und $\sigma=$ $0.5\left(h_{1}\right), \sigma=1\left(h_{2}\right), \sigma=2\left(h_{3}\right)$ 
Modells (drei Parameter). Daher wird dieses Modell häufig zur Modellsuche verwendet; vgl. Abschnitt 5.3. Das verallgemeinerte Gamma-Modell ermöglicht die Modellierung vieler Formen der Hazardfunktion, die mit den oben angeführten Modellen nicht abgebildet werden können - wie beispielsweise einen $U$-förmigen oder badewannenförmigen Verlauf, der das Abbruchrisiko von Studierenden oder das Sterberisiko von Menschen gut beschreibt. Der Grund, warum das verallgemeinerte Gamma-Modell meist nicht als endgültiges Modell verwendet wird, liegt einerseits in der komplizierten Formel der Hazardfunktion, bei der man im Gegensatz zu den einfacheren Modellen aus den geschätzten Parametern nicht sofort auf die Gestalt der Hazardfunktion schließen kann, und in der Rechenintensität der Maximum-Likelihood-Schätzung.

Beim verallgemeinerten Gamma-Modell folgt $\epsilon$ der Log-Gamma-Verteilung. Die Dichtefunktion von $T$ kann nach entsprechender Reparametrisierung des standardmäßig verwendeten verallgemeinerten Gamma-Modells angeschrieben werden als:

$$
f(t \mid x)=\frac{\kappa^{\kappa-1 / 2}}{\sigma t \Gamma(\kappa)} \exp \left\{\sqrt{\kappa} \frac{\log (t)-\mu(\mathbf{x})}{\sigma}-\kappa \exp \left(\frac{\log (t)-\mu(\mathbf{x})}{\sigma \sqrt{\kappa}}\right)\right\},
$$

wobei $\Gamma(\kappa)$ die Gammafunktion und der Formparameter $\kappa$ eine reelle Zahl größer Null ist. Lawless (1980) begründet die Reparametrisierung: “... studied the model in a different but equivalent form, which makes the properties and potential difficulties with estimation in the model much more transparent." In SAS und TDA wird ebenfalls diese Parametrisierung verwendet; siehe auch Rohwer und Pötter (1998) und SAS/STAT User' Guide, The LIFEREG Procedure. Die Überlebensfunktion ist

$$
S(t \mid \mathbf{x})=1-\int_{0}^{q(t)} \frac{u^{\kappa-1}}{\Gamma(\kappa)} \exp (-u) d u
$$

wobei $q(t)=\kappa \exp \{(\log (t)-\mu(\mathbf{x})) / \sigma \sqrt{\kappa}\}$ ist. Das Integral wird als unvollständige Gammafunktion bezeichnet. Als Spezialfälle des verallgemeinerten Gamma-Modells erhält man mit

- $\kappa=1$ und $\sigma=1$ das Exponential-Modell;

- $\kappa=1$ das Weibull-Modell;

- $\kappa \rightarrow \infty$ das log-normale Modell;

- $\sigma \sqrt{\kappa}=1$ das standardisierte Gamma-Modell. 


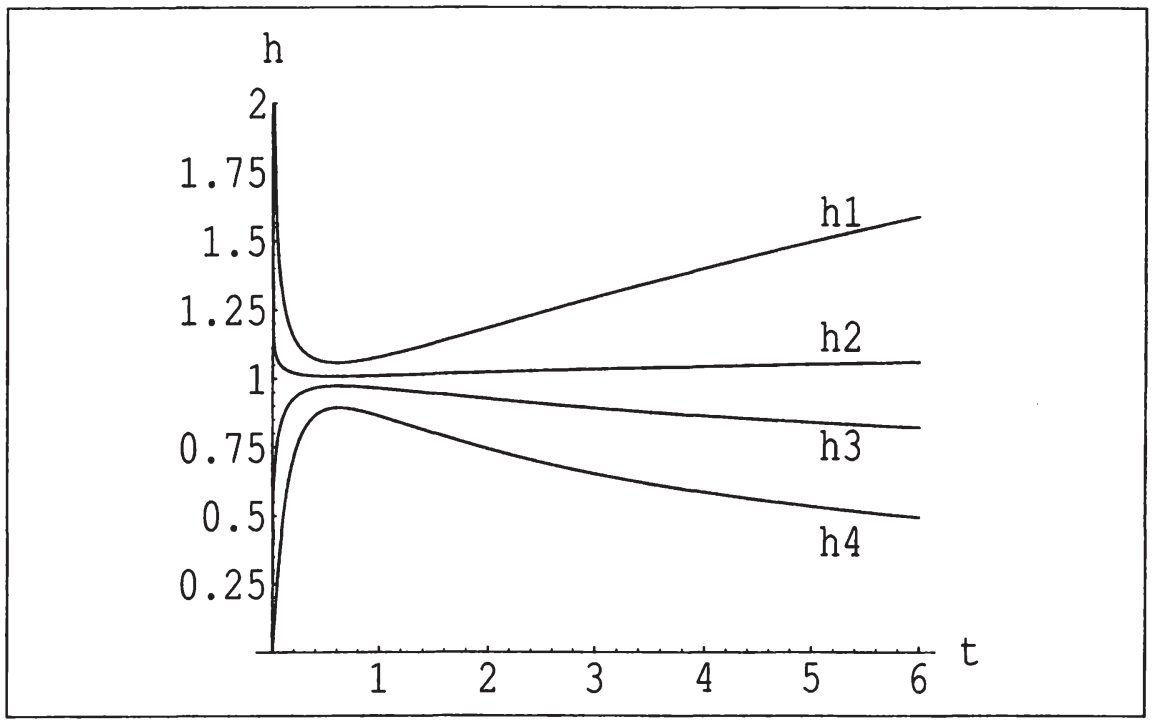

Abbildung 5.5: Hazardraten des verallgemeinerten Gamma-Modells für $\mu=$ $0, \sigma=1$ und $\kappa=0.5\left(h_{1}\right), \kappa=0.9\left(h_{2}\right), \kappa=1.5\left(h_{3}\right), \kappa=10\left(h_{4}\right)$

Mit Hilfe dieser Beziehungen kann das verallgemeinerte Gamma-Modell verwendet werden, um die Güte der Anpassung an die Daten der anderen Modelle zu überprüfen; siehe Abschnitt 5.3. Abbildung 5.5 zeigt Hazardfunktionen der verallgemeinerten Gamma-Verteilung für verschiedene $\kappa$-Werte.

Die hier beschriebenen log-linearen Ereignisdatenmodelle werden beispielsweise in Allison (1998), Klein und Moeschberger (1997) sowie in Rohwer und Pötter (1998) behandelt. In der Literatur (und bei der Verwendung von Computerprogrammen) ist auf die unterschiedlichen Parametrisierungen der besprochenen Modelle zu achten; siehe etwa Collett (1994). Die vorliegende Arbeit hält sich an die Parametrisierungen der Software SAS, wo das Interzept $\beta_{0}$, die Regressionskoeffizienten $\boldsymbol{\beta}$, der Skalenparameter $\sigma$ und im verallgemeinerten Gamma-Modell der Parameter $\delta$ geschätzt wird, wobei $\delta$ anstelle von $\kappa=\delta^{-2}$ verwendet wird.

\section{Proportionale Hazards-Modelle}

Neben der Klasse der log-linearen Modelle ist die Klasse der von Cox (1972) vorgeschlagenen proportionalen Hazards-Modelle (PH-Modelle) in der Ereig- 


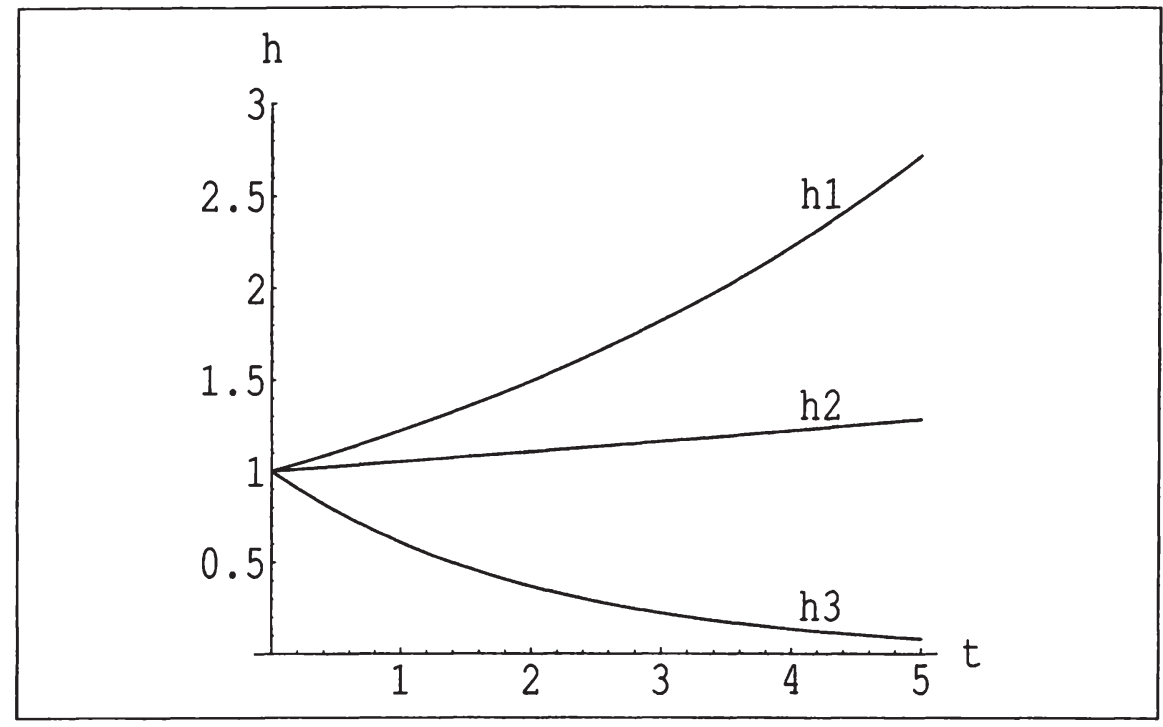

Abbildung 5.6: Hazardraten des Gompertz-Modells für $\exp \left(\theta_{0}\right)=1$ und $\gamma=$ $0.2\left(h_{1}\right), \gamma=0.05\left(h_{2}\right), \gamma=-0.5\left(h_{3}\right)$

nisdatenanalyse von Bedeutung. Für diese Modelle gilt:

$$
h(t \mid \mathbf{x})=h_{0}(t) g(\mathbf{x} ; \boldsymbol{\theta}),
$$

wobei meist $g(\mathbf{x} ; \boldsymbol{\theta})=\exp \left(\boldsymbol{x}^{\prime} \boldsymbol{\theta}\right)$ gewählt wird. Die Kovariablen wirken multiplikativ auf die Basis-Hazardfunktion $h_{0}(t)$. Nach dieser Definition zählen das Exponential- und das Weibull-Modell auch zu den PH-Modellen. Für das Weibull-Modell gilt beispielsweise:

$$
\begin{aligned}
h(t \mid \mathbf{x}) & =h_{0}(t) \exp \left(\boldsymbol{x}^{\prime} \boldsymbol{\theta}\right)=\delta \lambda^{\delta} t^{\delta-1} \exp \left(\boldsymbol{x}^{\prime} \boldsymbol{\theta}\right) \\
& =\delta t^{\delta-1}\left[\exp \left(-\beta_{0}-\boldsymbol{x}^{\prime} \boldsymbol{\beta}\right)\right]^{\delta}
\end{aligned}
$$

wobei $\lambda=\exp \left(-\beta_{0}\right)$ und $\boldsymbol{\beta}=-\boldsymbol{\theta} / \delta$.

Ein weiterer wichtiger Vertreter der PH-Modelle ist das Gompertz-Modell. Die Hazardfunktion ist mit

$$
h(t \mid \mathbf{x})=\exp \left(\theta_{0}+\boldsymbol{x}^{\prime} \boldsymbol{\theta}\right) \exp (\gamma t)
$$

gegeben. Abbildung 5.6 zeigt Hazardfunktionen der Gompertz-Verteilung für verschiedene $\gamma$-Werte. 


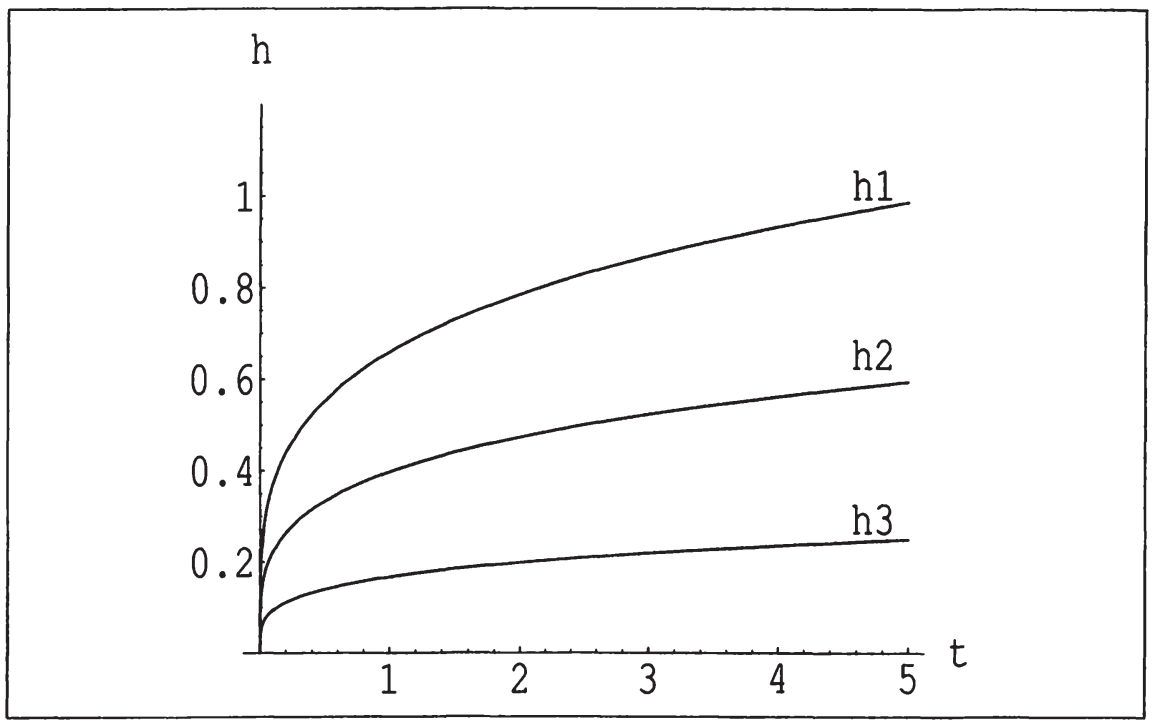

Abbildung 5.7: Proportionale Hazardraten der Weibull-Verteilung

Für alle Modelle der PH-Klasse gilt die Eigenschaft der "proportionalen Hazards", die besagt, dass die Hazardrate von einem Individuum mit Kovariablenvektor $\mathbf{x}_{i}$ ein Vielfaches der Rate eines anderen Individuums (mit $\mathbf{x}_{j}$ ) ist; das Vielfache ist eine Funktion von $\left(\mathbf{x}_{i}-\mathbf{x}_{j}\right)$. Bei den log-linearen Modellen gilt diese Eigenschaft nicht. Ausführlich wird das (semiparametrische) proportionale Hazards-Modell und seine Eigenschaften im Kapitel 6 behandelt. Abbildung 5.7 zeigt die proportionalen Hazardfunktionen der Weibull-Verteilung für verschiedene Werte einer Kovariablen.

Die allgemeinen Darstellungen (5.4) und (5.5) beider Klassen enthalten den Term $h_{0}(t)$, die beliebige Basis-Hazardfunktion. Wird $h_{0}(t)$ spezifiziert, dann erhält man die oben besprochenen parametrischen Regressionsmodelle. Wird $h_{0}(t)$ nicht näher spezifiziert, spricht man von einem semiparametrischen Ansatz. Für proportionale Hazards-Modelle sind geeignete Methoden zur Schätzung der unbekannten Regressionskoeffizienten entwickelt worden, die ohne nähere Spezifikation von $h_{0}(t)$ auskommen. Diese Methoden werden im Kapitel 6 behandelt.

Neben den log-linearen- und proportionalen (multiplikativen) HazardratenModellen können auch additive Hazardraten-Modelle der Form

$$
h(t \mid \mathbf{x})=h_{0}(t)+\sum_{j=1}^{p} x_{j}(t) \beta_{j}(t)
$$


zur Modellierung der Kovariableneffekte verwendet werden. Additive Hazardraten-Modelle werden in der vorliegenden Arbeit nicht behandelt; einen Überblick geben beispielsweise Hosmer und Lemeshow (1999) sowie Klein und Moeschberger (1997).

\subsection{Schätzen der Parameter und Hypothesen- tests}

\section{Maximum-Likelihood-Schätzung}

Die unbekannten Regressionskoeffizienten werden in dieser Arbeit in Folge für alle Regressionsmodelle mit $\boldsymbol{\beta}$ bezeichnet. Zur Schätzung der Regressionskoeffizienten der im vorherigen Abschnitt diskutierten parametrischen Regressionsmodelle wird im allgemeinen die Maximum-Likelihood-Methode (MLMethode) herangezogen; auch in der gängigen Statistik-Software ist (ausschließlich) diese Methode zur Schätzung der Parameter implementiert. KleinsteQuadratsummen-Schätzer werden im Rahmen der Ereignisdatenanalyse kaum behandelt. In dieser Arbeit wird daher nur auf die ML-Schätzmethode eingegangen.

Die ML-Methode ist deshalb so populär, da die Schätzer ein gutes asymptotisches Verhalten aufweisen. Unter gewissen Regularitätsbedingungen (siehe etwa Andersen et al. (1993) für die mathematischen Details) sind die MLSchätzer der Parameter konsistent, asymptotisch effizient und asymptotisch normalverteilt. Weiters ermöglicht die ML-Methode die Einbeziehung (rechtszensierter Daten bei der Schätzung der Parameter.

Zur Konstruktion der Likelihoodfunktion unter Einbeziehung von (bedingt) unabhängig rechts-zensierten Daten (siehe Abschnitt 3.2) wird der Zensierungsindikator $\delta_{i}$ wie folgt definiert:

$$
\delta_{i}= \begin{cases}1 & \text { falls } t_{i} \text { eine Ereigniszeit ist } \\ 0 & \text { falls } t_{i} \text { eine Zensierungszeit ist }\end{cases}
$$

Unter der Annahme, dass die Zensierungszeiten bedingt unabhängig von den Ereigniszeiten sind, gilt für nicht-zensierte Daten

$$
P(T=t, \delta=1 \mid \mathbf{x})=f(t \mid \mathbf{x})[1-G(t \mid \mathbf{x})]
$$

und für zensierte Fälle

$$
P(T=t, \delta=0 \mid \mathbf{x})=g(t \mid \mathbf{x})[1-F(t \mid \mathbf{x})]=g(t \mid \mathbf{x}) S(t \mid \mathbf{x}),
$$


wobei $f(t \mid \mathbf{x})$ und $F(t \mid \mathbf{x})$ Wahrscheinlichkeitsdichte und Verteilungsfunktion für die Zeitdauer bis zum Ereignis sind, sowie $g(t \mid \mathbf{x})$ und $G(t \mid \mathbf{x})$ Wahrscheinlichkeitsdichte und Verteilungsfunktion der Dauer bis zur Zensierung sind bei gegebenem Kovariablenvektor $\mathbf{x}$.

Somit erhalten wir folgende Likelihoodfunktion

$$
\begin{aligned}
L(\boldsymbol{\beta}) & =\prod_{i=1}^{n}\left\{f\left(t_{i} \mid \mathbf{x}_{i}\right)\left[1-G\left(t_{i} \mid \mathbf{x}_{i}\right)\right]\right\}^{\delta_{i}}\left\{g\left(t_{i} \mid \mathbf{x}_{i}\right)\left[S\left(t_{i} \mid \mathbf{x}_{i}\right)\right]\right\}^{1-\delta_{i}} \\
& =\prod_{i=1}^{n}\left\{f\left(t_{i} \mid \mathbf{x}_{i}\right)^{\delta_{i}}\left[S\left(t_{i} \mid \mathbf{x}_{i}\right)\right]^{1-\delta_{i}}\right\}\left\{g\left(t_{i} \mid \mathbf{x}_{i}\right)^{1-\delta_{i}}\left[1-G\left(t_{i} \mid \mathbf{x}_{i}\right)\right]^{\delta_{i}}\right\},
\end{aligned}
$$

wobei $f\left(t_{i} \mid \mathbf{x}_{i}\right)\left[1-G\left(t_{i} \mid \mathbf{x}_{i}\right)\right]$ der Beitrag des Individuums $i$ zur Likelihoodfunktion ist, falls die Beobachtung nicht zensiert ist; der Beitrag einer zensierten Beobachtung zur Likelihoodfunktion ist $g\left(t_{i} \mid \mathbf{x}_{i}\right)\left[S\left(t_{i} \mid \mathbf{x}_{i}\right)\right]$.

Unter der Annahme, dass die Verteilung der Zensierungszeiten nicht von den für $f_{i}$ und $S_{i}$ relevanten Parametern abhängt, so kann der zweite Faktor in (5.6) zu einem (hinsichtlich $\boldsymbol{\beta}$ ) konstanten Term zusammengefasst werden und der ML-Schätzer durch Maximieren der linken Seite von (5.6), also

$$
\begin{aligned}
L(\boldsymbol{\beta}) & =\prod_{i=1}^{n} f\left(t_{i} \mid \mathbf{x}_{i}\right)^{\delta_{i}}\left[S\left(t_{i} \mid \mathbf{x}_{i}\right)\right]^{1-\delta_{i}} \\
& =\prod_{i=1}^{n} h\left(t_{i} \mid \mathbf{x}_{i}\right)^{\delta_{i}} \exp \left(-\int_{0}^{t_{i}} h\left(u \mid \mathbf{x}_{i}\right) d u\right),
\end{aligned}
$$

erhalten werden. In diesem Fall spricht man von einem nicht-informativen Zensierungsmechanismus. Typ I und II rechts-zensierte Fälle sind nicht-informative zensierte Daten. Kalbfleisch und Prentice (1980) meinen zu dieser Bedingung: "Realistic examples of informative, but independent, censoring schemes are hard to construct". Ausführlich werden (bedingt) unabhängige Zensierungsmechanismen etwa von Kalbfleisch und Prentice (1980) und Lagakos (1979) diskutiert.

Für die ML-Schätzung wird dann meist, da rechentechnisch einfacher, die LogLikelihoodfunktion nach den unbekannten Parametern maximiert:

$$
l(\boldsymbol{\beta})=\ln L(\boldsymbol{\beta})=\sum_{i=1}^{n}\left(\delta_{i} \ln h\left(t_{i} \mid \mathbf{x}_{i}\right)-\int_{0}^{t_{i}} h\left(u \mid \mathbf{x}_{i}\right) d u\right)
$$

Die Maximierung geschieht in der Regel mit Hilfe eines iterativen Verfahrens, etwa des Newton-Verfahrens oder eines modifizierten Newton-Verfahrens (wie in SAS; siehe Allison (1998)). Die Herleitung der Log-Likelihoodfunktion und 
der ML-Gleichungen für das Exponential-Regressionsmodell finden sich in zahlreichen Monographien, zum Beispiel in Allison (1998). Für alle anderen im Abschnitt 5.1 diskutierten parametrischen Regressionsmodelle sind diese Herleitungen zum Beispiel in Rohwer und Pötter (1998) zu finden.

Manchmal tritt bei den iterativen Verfahren keine Konvergenz auf, insbesondere für

- sehr kleine Stichprobengrößen,

- eine große Anzahl von Parametern oder

- eine große Anzahl zensierter Fälle.

Einen Überblick über die allgemeine Theorie der ML-Methode und ihre Verwendung im Zusammenhang mit Ereignisdatenmodellen geben etwa Blossfeld et al. (1986) und Kalbfleisch und Prentice (1980).

\section{Tests für Regressionskoeffizienten}

Analog zur multiplen linearen Regression können Hypothesen über die Parameter formuliert werden. Im Allgemeinen wird überprüft, ob die in das Modell einbezogenen Variablen insgesamt einen Erklärungsbeitrag liefern; es wird dann die Nullhypothese getestet, dass alle Parameter gleich Null sind. Zusätzlich kann für jede Variable einzeln getestet werden, ob ihr Koeffizient $\beta_{j}$ unter Berücksichtigung der anderen im Modell inkludierten Variablen den Wert Null hat. Eine analoge Aufgabenstellung ist es, Konfidenzintervalle für die Parameterschätzer zu berechnen.

Zum Testen von Hypothesen über die Parameter $\beta_{1}, \cdots, \beta_{p}$ werden standardmäßig drei - asymptotisch äquivalente - Tests eingesetzt: der LikelihoodQuotienten-, der Wald- und der Score-Test.

Die - einfach zu berechnende - Likelihood-Quotienten-Teststatistik zum Testen der globalen Hypothese $H_{0}: \boldsymbol{\beta}=\boldsymbol{\beta}_{0}$, wobei $\boldsymbol{\beta}=\left(\beta_{1}, \cdots, \beta_{p}\right)^{\prime}$ die Parameter im Modell sind und $\boldsymbol{\beta}_{0}$ ein reeller Vektor ist, erhält man mit

$$
G=2\left[l(\hat{\boldsymbol{\beta}})-l\left(\boldsymbol{\beta}_{0}\right)\right]
$$

wobei $l($.$) der Wert der logarithmierten Likelihoodfunktion ist.$ 
Zur Durchführung der beiden anderen Tests benötigt man einen Schätzer der Kovarianzmatrix von $\hat{\boldsymbol{\beta}}$

$$
\widehat{\operatorname{Var}}(\hat{\boldsymbol{\beta}})=\left(-\frac{\partial^{2} l}{\partial \boldsymbol{\beta}^{2}}(\hat{\boldsymbol{\beta}})\right)^{-1}=\mathbf{I}(\hat{\boldsymbol{\beta}})^{-1},
$$

wobei $\mathbf{I}(\boldsymbol{\beta})$ als beobachtete Informationsmatrix bezeichnet wird. Die WaldTeststatistik erhält man mit

$$
W=\left(\hat{\boldsymbol{\beta}}-\boldsymbol{\beta}_{0}\right)^{\prime} \mathbf{I}(\hat{\boldsymbol{\beta}})\left(\hat{\boldsymbol{\beta}}-\boldsymbol{\beta}_{0}\right)
$$

und die Score-Teststatistik mit

$$
S=\mathbf{u}\left(\boldsymbol{\beta}_{0}\right)^{\prime}\left[\mathbf{I}\left(\boldsymbol{\beta}_{0}\right)\right]^{-1} \mathbf{u}\left(\boldsymbol{\beta}_{0}\right),
$$

wobei $\mathbf{u}\left(\boldsymbol{\beta}_{0}\right)$ der Vektor der ersten partiellen Ableitungen der logarithmierten Likelihoodfunktion an der Stelle $\boldsymbol{\beta}_{0}$ ist.

Alle drei Teststatistiken sind bei Gültigkeit der Nullhypothese asymptotisch $\chi^{2}(p)$ verteilt. In Anwendungsbeispielen liefern die drei Teststatistiken (5.8), (5.9) und (5.10) ähnliche numerische Werte und führen daher (meist) zu gleichen Schlüssen.

Als Spezialfall erhält man für die Hypothese, dass alle Parameterschätzer gleich Null sind $\left(H_{0}: \boldsymbol{\beta}=\mathbf{0}\right)$, die Likelihood-Quotienten-Teststatistik mit

$$
G=2[l(\hat{\boldsymbol{\beta}})-l(\mathbf{0})] .
$$

Die Tests können auch zum Überprüfen von Hypothesen über einen Teil der Parameter herangezogen werden; siehe Klein und Moeschberger (1997). Ein wichtiger Spezialfall ist die Überprüfung der Hypothese, dass eine Variable unter Berücksichtigung der anderen in das Modell einbezogenen Variablen keinen Effekt hat $\left(H_{0}: \beta_{j}=0\right)$. Hierfür bietet sich die (univariate) WaldTeststatistik

$$
W=\frac{\hat{\beta}_{j}}{\sqrt{\widehat{\operatorname{Var}}\left(\hat{\beta}_{j}\right)}}
$$

an, die bei Gültigkeit von $H_{0}$ asymptotisch standard-normalverteilt ist. Die Endpunkte eines $100(1-\alpha) \%$-Konfidenzintervalls für $\beta_{j}$ erhält man mit

$$
\hat{\beta}_{j} \pm z_{1-\alpha / 2} \sqrt{\widehat{\operatorname{Var}}\left(\hat{\beta}_{j}\right)}
$$

wobei $z_{1-\alpha / 2}$ das $(1-\alpha / 2)$-Quantil der Standard-Normalverteilung ist. 
Die Hypothese $\beta_{i}=\beta_{j}$ auf Gleichheit von zwei Regressionskoeffizienten kann analog mit der Teststatistik

$$
W=\frac{\hat{\beta}_{i}-\hat{\beta}_{j}}{\sqrt{\widehat{\operatorname{Var}}\left(\hat{\beta}_{i}-\hat{\beta}_{j}\right)}}
$$

überprüft werden.

\section{3 Überprüfung von Verteilungsannahmen}

\section{Hypothesentests bei geschachtelten Modellen}

Im Abschnitt 5.1 wurden einige Verteilungsannahmen für die Dauer $T$ bis zum Eintreffen des Ereignisses diskutiert. In konkreten Anwendungsfällen ist es wünschenswert, die Anpassungsgüte von unterschiedlichen Modellen an die Daten zu testen und untereinander zu vergleichen. Dieser Abschnitt behandelt Methoden, die dem Anwender die Entscheidung für (oder gegen) ein parametrisches Modell erleichtern soll.

Im vorherigen Abschnitt sind mit (5.8) die Likelihood-Quotienten-, mit (5.9) die Wald- und mit (5.10) die Score-Teststatistik definiert worden. Diese Teststatistiken werden zum Testen von Hypothesen über Parameterrestriktionen in einem implementierten Modell verwendet. Die durch die Parameterrestriktionen erhaltenen Modelle sind Spezialfälle des allgemeineren Modells (geschachtelte Modelle, engl. nested models). So ist etwa ein Regressionsmodell ohne Kovariablen ein Spezialfall des gleichen Modells, das diese Kovariablen berücksichtigt. Den Spezialfall erhält man durch die Parameterrestriktionen $\beta_{1}=\beta_{2}=\cdots=\beta_{p}=0$. Ebenso erhält man durch die Hypothese $\beta_{j}=0$ einen Spezialfall des allgemeinen Modells, das auch die Kovariable $x_{j}$ berücksichtigt. Im Abschnitt 5.1 ist gezeigt worden, dass im verallgemeinerten Gamma-Modell das standardisierte Gamma-Modell, das Weibull-, das Exponential- und das log-normale Modell enthalten sind. Daher können die besprochenen Test-Statistiken für einen formalen Vergleich dieser Modelle mit dem verallgemeinerten Gamma-Modell verwendet und die Frage geklärt werden, ob das allgemeinere Modell eine bessere Anpassung an die Daten als das eingeschränkte Modell hat. Wird etwa im verallgemeinerten Gamma-Modell die Hypothese $H_{0}: \kappa=1$ verworfen, so beschreibt das verallgemeinerte Gamma-Modell die Daten besser als das Weibull-Modell.

Das log-logistische Modell hingegen ist kein Spezialfall des verallgemeinerten Gamma-Modells. Eine Möglichkeit, parametrische Modelle, die nicht geschach- 
telt sind, miteinander zu vergleichen, bietet das von Akaikie (1974) vorgeschlagene Kriterium (engl. Akaikie information criterion)

$$
A I C=-2 l+2(p+1+k),
$$

wobei $l$ der Log-Likelihood-Wert, $p$ die Anzahl der Kovariablen und $k=0$ für das Exponential-Modell, $k=1$ für das Weibull-, log normale, StandardGamma-, log logistische Modell und $k=2$ für das verallgemeinerte GammaModell ist. Das Modell, das den kleinsten AIC-Wert hat, wird als Modell mit der besten Anpassung an die Daten gesehen.

Bei der Interpretation der Teststatistiken muss darauf geachtet werden, dass das verallgemeinerte Gamma-Modell selbst nicht mehr mit einem allgemeineren Modell verglichen werden kann und daher die vergleichenden Aussagen von der Anpassungsgüte des verallgemeinerten Gamma-Modells abhängen.

\section{Graphische Verfahren}

Meist werden graphische Methoden verwendet, um die Modelle auf ihre Anpassungsgüte zu überprüfen. Diese graphischen Verfahren geben gute Hinweise, welche Modelle weniger oder besser geeignet zur Beschreibung der Daten sind; sie sind jedoch keine formale Überprüfung, ob ein bestimmtes parametrisches Modell das geeignete ist.

Um die Anpassungsgüte von Modellen ohne Berücksichtigung von Kovariablen zu untersuchen, werden Transformationen der Überlebensfunktion gesucht, die linear in $g(t)$ sind, wobei $g(t)$ eine beliebige Funktion der Zeit $t$ ist. Dazu wird die Überlebensfunktion erst mit einer nicht-parametrischen Methode wie der Kaplan-Meier-, Sterbetafel- oder Nelson-Aalen-Methode geschätzt (siehe Kapitel 4) und die transformierten Werte der geschätzten Überlebensfunktion gegen $g(t)$ aufgetragen. Bei guter Anpassung an die Daten sollte die Graphik nicht viel von einer Geraden abweichen. Diese Methode soll nun für das Exponential-, das Weibull-, das log-normale und das log-logistische Modell erklärt werden.

Im Exponential-Modell führt die Logarithmus-Transformation die Überlebensfunktion $S(t)=\exp (-\lambda t)$ in

$$
-\ln [S(t)]=\lambda t
$$

über; ein Plot von $-\ln [\hat{S}(t)]$ gegen $t$, wobei $\hat{S}(t)$ eine nicht-parametrische Schätzfunktion der Überlebensfunktion ist, sollte daher von einer Geraden durch den Ursprung nicht sehr abweichen, falls die Annahme eines ExponentialModells passend ist. 
Im Weibull-Modell kann die Überlebensfunktion $S(t)=\exp \left(-(t \lambda)^{\delta}\right)$ mit

$$
\ln (\ln [-S(t)])=\delta \ln (\lambda)+\delta \ln (t)
$$

in eine lineare Funktion von $\ln (t)$ transformiert werden; ein Plot von $\ln (\ln [-\hat{S}(t)])$ gegen $\ln (t)$ müsste daher annähernd linear sein, falls die Annahme eines WeibullModells passend ist.

Die Überlebensfunktion $S(t)=1 /\left(1+(\lambda t)^{\delta}\right)$ im log-logistischen Modell kann mit

$$
\ln \left(\frac{1-S(t)}{S(t)}\right)=\delta \ln (\lambda)+\delta \ln (t)
$$

in eine lineare Funktion (von $\ln (t)$ ) übergeführt werden. Deshalb müsste ein Plot von $\ln [(1-\hat{S}(t)) / \hat{S}(t)]$ gegen $\ln (t)$ annähernd linear sein, falls die Annahme eines log-logistischen Modells passend ist.

Im $\log$-normalen Modell wird die Funktion $S(t)=1-\Phi[(\log (t)-\mu) / \sigma]$ mit

$$
\Phi^{-1}[1-S(t)]=-\frac{\mu}{\sigma}+\frac{1}{\sigma} \ln (t)
$$

in eine lineare Funktion übergeführt. Dementsprechend müsste ein Plot von $\Phi^{-1}[1-\hat{S}(t)]$ gegen $\ln (t)$ annähernd linear sein, falls das log-normale Modell eine gute Anpassung an die Daten liefert.

Die Anpassungsgüte der einzelnen Modelle kann grob "mit freiem Auge" abgeschätzt werden, da Abweichungen von einer Geraden meist leicht zu erkennen sind. Ergänzend kann auch ein lineares Regressionsmodell geschätzt werden und mit Hilfe von $R^{2}$ die Anpassungsgüte überprüft werden. Bei der Interpretation dieser Überprüfungen muss jedoch darauf geachtet werden, dass für die Überprüfung der Anpassungsgüte keine Kovariablen in das Modell einbezogen worden sind, das heißt eine homogene Population vorausgesetzt worden ist. Vernachlässigte Heterogenität kann jedoch die Gestalt der Überlebensfunktion stark beeinflussen, sodass Modell-Entscheidungen, die auf Modelle ohne Berücksichtigung von Kovariablen beruhen, nicht überbewertet werden dürfen; siehe Abschnitt 6.3.

Zur (graphischen) Überprüfung der Adäquatheit eines log-linearen Ereignisdatenmodells (für zwei Gruppen mit $(0,1)$-Kodierung) können auch Q-Q Plots (Quantile-Quantile Plots) herangezogen werden. Aufgrund der Beziehung (5.3) sind die Quantile $t_{0}(p)$ der Gruppe 0 ein Vielfaches der Quantile $t_{1}(p)$ der zweiten Gruppe. Ein Plot von $t_{0}(p)$ gegen $t_{1}(p)$ für verschiedene $p$-Werte sollte bei Adäquatheit des gewählten log-linearen Modelles annähernd linear durch den Ursprung sein; siehe etwa Collett (1994). 
Zur Überprüfung der Anpassungsgüte eines parametrischen Ereignisdatenmodells können die sogenannten Cox-Snell-Residuen herangezogen werden, die mit

$$
\hat{e}_{i}=-\ln \hat{S}\left(t_{i} \mid \mathbf{x}_{i}\right)=\hat{H}\left(t_{i} \mid \mathbf{x}_{i}\right),
$$

definiert sind, wobei $t_{i}$ die beobachtete Ereignis- oder Zensierungszeit des Individuums $i$ ist.

Über die Tatsache, dass eine Zufallsvariable $Y=-\ln S(T)$ unabhängig von der Gestalt der Überlebensfunktion $S(t)$ eine Exponentialverteilung mit Erwartungswert Eins besitzt (siehe etwa Collett (1994)), kann folgende Aussage getroffen werden: Hat das betrachtete Modell eine gute Anpassungsgüte, dann sind die $\hat{e}_{i}$ 's annähernd exponentialverteilt mit Parameter $\lambda=1$. Ist $t_{i}$ eine zensierte Verweildauer, so wird das zugehörige Residuum ebenfalls als zensiert betrachtet. Ein Plot von $-\ln [\hat{S}(\hat{e})]$ gegen $\hat{e}$, wobei $\hat{S}(e)$ eine nicht-parametrische Schätzfunktion ist, sollte daher annähernd eine Gerade durch den Ursprung ergeben. Näher wird auf Residuenanalysen im Abschnitt 6.5 eingegangen.

Die Überprüfung der Anpassungsgüte von parametrischen Regressionsmodellen wird beispielsweise in Allison (1998), Blossfeld und Rohwer (1995), Collett (1994) sowie Klein und Moeschberger (1997) behandelt.

\subsection{Das stückweise konstante Hazards-Modell}

Das im Abschnitt 5.1 diskutierte Exponential-Modell ist aufgrund der Annahme einer konstanten Hazardrate in praktischen Anwendungen meist nicht geeignet. Das stückweise konstante Hazards-Modell ist eine Verallgemeinerung des Exponential-Modells und wird sehr häufig zur Modellierung von Ereignisdaten verwendet. Zur Modellierung des stückweise konstanten Modells wird die Beobachtungsperiode in beliebig viele (nicht notwendigerweise gleich lange) Intervalle geteilt, in denen jeweils eine exponentialverteilte Verweildauer angenommen wird. Die Hazardrate im jeweiligen Intervall ist somit konstant. Neben der mathematischen Einfachheit des Exponential-Modells hat dieses Modell die Vorteile, dass

- die Hazardrate im Gegensatz zum einfachen Exponential-Modell mit der Zeit variieren kann - nämlich zwischen fixen (beliebig gewählten) Perioden - und somit die Abhängigkeit der Hazardfunktion von der Zeit analysiert werden kann;

- die Zeitabhängigkeit von Kovariablen, deren Werte im Laufe des Prozesses variieren können, in Anwendungsbeispielen einfach modelliert werden 
kann (zeitvariierende Kovariablen werden ausführlich im nächsten Kapitel, Abschnitt 6.3, diskutiert);

- auch die Effekte der Kovariablen zwischen den Perioden variieren können.

Ein Nachteil dieses Modells ist, dass die Willkürlichkeit der Intervallgrenzen die Schätzung beeinflussen kann.

Werden mit $a_{0}, a_{1}, \ldots, a_{N}$, wobei $N$ die beliebige Anzahl der Intervalle ist und $a_{0}=0, a_{N}=\infty$ gilt, die Grenzen zwischen den Intervallen bezeichnet, so erhält man für das stückweise konstante Modell mit zeitkonstanten Effekten der Kovariablen die Hazardfunktion im $r$-ten Intervall $\left[a_{r-1}, a_{r}\right), r=1, \ldots, N$, mit

$$
h_{r}(\mathbf{x})=h(t \mid \mathbf{x})=\exp \left(-\beta_{r 0}-\boldsymbol{x}^{\prime} \boldsymbol{\beta}\right) \text { für } a_{r-1} \leq t<a_{r} .
$$

Die Hazardrate ist innerhalb der Intervalle konstant und ändert sich zwischen den Intervallen durch den Parameter $\beta_{r 0}$. Erlebt das Individuum $i$ im $N_{i}$-ten Intervall, $N_{i} \leq N$, zum Zeitpunkt $t_{i}$ ein Ereignis oder wird es in diesem Intervall rechts-zensiert, so erhält man die Likelihoodfunktion des Modells (5.11):

$$
L(\boldsymbol{\beta})=\prod_{i=1}^{n} \prod_{r=1}^{N_{i}}\left\{h_{r}\left(\mathbf{x}_{i}\right)\right\}^{\delta_{i r}} \exp \left(-h_{r}\left(\mathbf{x}_{i}\right) u_{i r}\right),
$$

wobei

$$
\begin{aligned}
& u_{i r}= \begin{cases}a_{r}-a_{r-1} & r=1, \ldots, N_{i}-1 \\
t_{i}-a_{N_{i}-1} & r=N_{i}\end{cases} \\
& \delta_{i r}= \begin{cases}1 & r=N_{i} \text { und das } i \text {-te Individuum hat zu } t_{i} \text { ein Ereignis } \\
0 & r=N_{i} \text { und das } i \text {-te Individuum ist rechts-zensiert } \\
0 & r=1, \ldots, N_{i}-1\end{cases}
\end{aligned}
$$

Für die ML-Schätzung wird somit die Beobachtungsperiode jedes Individuums in $N_{i}$ Intervalle aufgeteilt. Trifft für ein Individuum ein Ereignis im dritten Intervall ein $\left(N_{i}=3\right)$ oder wird es in diesem Intervall zensiert, so werden aus dieser Beobachtung drei verschiedene Beobachtungen gebildet. Die ersten beiden Beobachtungen gehen als rechts-zensierte Fälle mit einer Zeitdauer, die der Länge des betreffenden Intervalls entspricht, in die Schätzung ein. Die dritte Beobachtung geht als Ereignis oder als rechts-zensierter Fall mit Zeitdauer von Beginn dieses Intervalls bis zum Ereignis oder zur Zensierung in die Schätzung ein.

Die Zeitabhängigkeit von Kovariablen, deren Werte im Laufe des Prozesses variieren können, kann insofern leicht berücksichtigt werden, indem für jedes Individuum bei der ML-Methode zu Beginn jedes Intervalls die Werte 
der zeitabhängigen Variablen aktualisiert werden. Eine (stetige oder diskrete) zeitvariierende Kovariable $x(t)$ nimmt somit im $r$-ten Intervall den konstanten Wert $x\left(a_{r-1}\right)$ an, das ist jener Wert, der zu Beginn des $r$-ten Intervalls beobachtet wurde. Da eine zeitvariierende Variable innerhalb eines jeden Intervalls als konstant angenommen wird, erfolgt die Schätzung der Parameter analog (5.12).

Zeitvariierende Effekte der Kovariablen werden im verallgemeinerten stückweise konstanten Modell

$$
h_{r}(\mathbf{x})=h(t \mid \mathbf{x})=\exp \left(-\beta_{r 0}-\boldsymbol{x}^{\prime} \boldsymbol{\beta}_{r}\right) \quad \text { für } a_{r-1} \leq t<a_{r}
$$

ermöglicht. Die Koeffizientenvektoren $\boldsymbol{\beta}_{r}$ können somit mit der Periode variieren. Es muss dann für jedes Intervall ein separates Exponential-Modell geschätzt werden.

In einer Analyse des Studienabbruch-Risikos an der Wirtschaftsuniversität Wien kann etwa vermutet werden, dass der Matura-Schultyp lediglich in den ersten Semestern einen Einfluss auf den Studienabbruch hat. Folgende Hypothese kann beispielsweise mit Hilfe des Modells (5.13) überprüft werden: "Maturanten von allgemein bildenden höheren Schulen sind in den ersten Semestern (aufgrund ihrer geringeren wirtschaftlichen Vorbildung) Abbruch gefährdeter als Maturanten von Handelsakademien. Dieser Effekt lässt jedoch nach und verschwindet nach einigen Semestern komplett."

Das Modell (5.13) ist äquivalent zu einem Modell, das in das Standard-Modell (5.11) alle möglichen Interaktionsvariablen zwischen den Perioden und den einbezogenen Kovariablen aufnimmt. Modell (5.11) ist ein Spezialfall vom verallgemeinerten Modell (5.13). Die Schätzung des Modells (5.13) unter der Restriktion $\boldsymbol{\beta}_{1}=\ldots=\boldsymbol{\beta}_{\boldsymbol{N}}$ führt zu gleichen Parameterschätzern wie die Schätzung des Modells (5.11).

Mit Hilfe des Likelihood-Quotiententests kann etwa die globale Hypothese $H_{0}$ : $\boldsymbol{\beta}_{1}=\ldots=\boldsymbol{\beta}_{\boldsymbol{N}}$ getestet werden, dass die Parametervektoren in allen Perioden gleich sind. Die entsprechende Likelihood-Quotientenstatistik lautet

$$
G=2\left[\sum_{r=1}^{N} l_{r}\left(\hat{\boldsymbol{\beta}}_{r}\right)-l(\hat{\boldsymbol{\beta}})\right],
$$

wobei $l_{r}\left(\boldsymbol{\beta}_{r}\right)$ die logarithmierte Likelihoodfunktion der Beobachtungen aus dem $r$-ten Intervall bei Annahme des Modells (5.13) und $l(\boldsymbol{\beta})$ die logarithmierte Funktion (5.12) ist. Unter $H_{0}$ ist die Teststatistik asymptotisch $\chi^{2}$ mit $(N-1) p$ Freiheitsgraden verteilt.

Das stückweise konstante Hazards-Modell ist den im Kapitel 8 behandelten diskreten Ereignisdatenmodellen sehr ähnlich. Der wesentliche Unterschied be- 
steht darin, dass im diskreten Modell zur Schätzung der Parameter nicht de] Zeitpunkt des Ereignisses oder der Zensierung herangezogen wird, sondern nu berücksichtigt wird, ob ein Ereignis (oder eine Zensierung) in einem bestimm. ten Intervall eingetroffen ist oder nicht.

Das stückweise konstante Hazards-Modell wird beispielsweise in Allison (1998) Blossfeld und Rohwer (1995) und Yamaguchi (1991) behandelt. Alle in diesem. Abschnitt besprochenen Verfahren werden durch die gängige Statistik-Softwar€ unterstützt. 


\section{Kapitel 6}

\section{Das semiparametrische Hazards-Modell von Cox}

Im Abschnitt 5.1 wurden parametrische proportionale Hazards-Modelle $h(t \mid \mathbf{x})$ $=h_{0}(t) \exp \left(\boldsymbol{x}^{\prime} \boldsymbol{\beta}\right)$ diskutiert, wobei für die Basis-Hazardfunktion $h_{0}(t)$ eine spezielle parametrische Form gewählt worden ist.

In diesem Kapitel wird zur Schätzung der unbekannten Parameter $\boldsymbol{\beta}$ die BasisHazardfunktion $h_{0}(t)$ nicht näher spezifiziert. Das hier betrachtete semiparametrische PH-Modell (meist nur als Cox-Modell bezeichnet) ist somit flexibler als die im vorangegangenen Kapitel besprochenen Ansätze. Es ist allerdings nicht möglich, Hypothesen über die Abhängigkeit der Hazardfunktion von der Zeit zu testen.

Die Hazardrate und die Überlebensfunktion im Cox-Modell werden im nächsten Abschnitt diskutiert. Zur Schätzung der Parameter muss die ML-Methode modifiziert werden; die modifizierte (partielle) Likelihood-Methode zur Schätzung der unbekannten Parameter wird im Abschnitt 6.2 behandelt. Neben der hohen Flexibilität des Cox-Modells, die durch die nicht näher spezifizierte BasisHazardfunktion erreicht wird, macht das technisch einfache (und von der gängigen Statistik-Software unterstützte) Einbeziehen von zeitabhängigen Variablen und zeitvariierenden Effekten der Kovariablen das Cox-Modell für Anwender sehr attraktiv. Zeitabhängige Variablen und ihre Integration in das Cox-Modell und in parametrische Regressionsmodelle werden im Abschnitt 6.3 erläutert. Das stratifizierte Cox-Modell, das beispielsweise bei Verletzung der Proportionalitätsannahme verwendet werden kann, wird im Abschnitt 6.4 behandelt. Der letzte Abschnitt des Kapitels diskutiert Methoden zur Überprüfung des Cox-Modells, wobei insbesondere auf die Überprüfung der Proportionalitätsannahme eingegangen wird. 


\subsection{Hazardrate und Überlebensfunktion im Cox-Modell}

Wie im Abschnitt 5.1 erwähnt ist für proportionale Hazards-Modelle charakteristisch, dass die Hazardrate faktorisiert wird in einen Term, der nur von der Zeit, und in einen Term, der nur von den Kovariablen abhängt. Die allgemeine Darstellung eines proportionalen Hazards-Modells ist demnach

$$
h(t \mid \mathbf{x})=h_{0}(t) g(\mathbf{x} ; \boldsymbol{\beta}), \quad g(.)>0 .
$$

Die (zeitunabhängigen) Kovariablen haben somit einen multiplikativen Effekt auf $h_{0}(t)$. Wird die Funktion $g(\mathbf{x} ; \boldsymbol{\beta})$ so gewählt, dass $g(\mathbf{x}=\mathbf{0} ; \boldsymbol{\beta})=1$ gilt, bezeichnet man die Funktion $h_{0}(t)$ als Basis-Hazardrate. Spezifiziert man $h_{0}(t)$ nicht näher, so spricht man von semiparametrischen Modellen.

Die für diese Modelle charakteristische Eigenschaft der proportionalen Hazards ergibt sich aus der Betrachtung der Quotienten für zwei Individuen mit den Kovariablenvektoren $\mathbf{x}_{i}$ und $\mathbf{x}_{j}$

$$
H R\left(t, \mathbf{x}_{i}, \mathbf{x}_{j}\right)=\frac{h\left(t \mid \mathbf{x}_{i}\right)}{h\left(t \mid \mathbf{x}_{j}\right)}=\frac{g\left(\mathbf{x}_{i} ; \boldsymbol{\beta}\right)}{g\left(\mathbf{x}_{j} ; \boldsymbol{\beta}\right)} .
$$

Der Quotient $H R$, der als Hazard-Verhältnis bezeichnet wird, hängt somit nicht von der Zeit $t$ ab; die Hazardrate des $j$-ten Individuums ist daher das $H R$-fache der Hazardrate des $i$-ten Individuums.

Im populären Cox-Modell, das in diesem Kapitel behandelt wird, gilt $g(\mathbf{x} ; \boldsymbol{\beta})=$ $\exp \left(\mathbf{x}^{\prime} \boldsymbol{\beta}\right)$, und daher

$$
\begin{aligned}
H R=\frac{h\left(t \mid \mathbf{x}_{i}\right)}{h\left(t \mid \mathbf{x}_{j}\right)} & =\frac{h_{0}(t) \exp \left(\mathbf{x}_{i}^{\prime} \boldsymbol{\beta}\right)}{h_{0}(t) \exp \left(\mathbf{x}_{j}^{\prime} \boldsymbol{\beta}\right)} \\
& =\exp \left[\left(\mathbf{x}_{i}-\mathbf{x}_{j}\right)^{\prime} \boldsymbol{\beta}\right] .
\end{aligned}
$$

Betrachten wir ein Cox-Modell, das nur die Variable Geschlecht - mit $x_{1}=1$ für Männer und $x_{0}=0$ für Frauen - in das Modell einbezieht, dann gilt über die gesamte Zeit des Prozesses

$$
H R\left(t, x_{1}, x_{0}\right)=\exp (\beta) .
$$

Das bedeutet, dass sich die Stärke des Einflusses aller in das Modell einbezogenen Variablen nicht mit der Zeit ändert. Ist etwa $\beta=\ln (2)$, dann haben Männer über die gesamte Zeit des Prozesses ein doppelt so hohes Risiko für ein 
Ereignis wie Frauen. Diese Annahme von proportionalen Hazardraten schränkt die Flexibilität des Cox-Modells ein. Alternativen bei Verletzung der Proportionalitätsannahme werden in den Abschnitten 6.3 und 6.4 und Methoden zur Überprüfung der Proportionalitätsannahme im Abschnitt 6.5 besprochen.

Die Überlebensfunktion im Cox-Modell lässt sich ermitteln zu

$$
S(t \mid \mathbf{x})=S_{0}(t)^{\exp \left(\mathbf{x}^{\prime} \boldsymbol{\beta}\right)} .
$$

Somit sind auch die logarithmierten Überlebensfunktionen von Individuen mit den Kovariablenvektoren $\mathbf{x}_{i}$ und $\mathbf{x}_{j}$ mit dem gleichen Faktor wie die Hazardraten proportional zueinander.

\subsection{Partielle Likelihood Schätzung}

Die Likelihoodfunktion für das Cox-Modell ist nach (5.7) (unter Annahme eines nicht-informativen Zensierungsmechanismus)

$$
L(\boldsymbol{\beta})=\prod_{i=1}^{n}\left[h_{0}\left(t_{i}\right) \exp \left(\mathbf{x}_{i} \boldsymbol{\beta}\right)\right]^{\delta_{i}} \exp \left\{-\int_{0}^{t_{i}} h_{0}(u) \exp \left(\mathbf{x}_{i}^{\prime} \boldsymbol{\beta}\right) d u\right\} .
$$

Diese Funktion enthält neben den unbekannten Parametern $\boldsymbol{\beta}$ auch die beliebige Basis-Hazardfunktion $h_{0}(t)$; darum kann diese Likelihoodfunktion nicht zur Schätzung der Parameter herangezogen werden.

Es seien $t_{(1)}<\ldots<t_{(k)}, k \leq n$, die geordneten Ereigniszeiten, $\mathbf{x}_{(1)}, \ldots, \mathbf{x}_{(k)}$ die entsprechenden Kovariablen-Vektoren und $R(t)$ die Risikomenge zum Zeitpunkt $t$, dann erhält man aus (6.1) durch Erweiterung

$$
L(\boldsymbol{\beta})=\prod_{i=1}^{k} \frac{\exp \left(\mathbf{x}_{(i)}^{\prime} \boldsymbol{\beta}\right)}{\sum_{l \in R\left(t_{(i)}\right)} \exp \left(\mathbf{x}_{l}^{\prime} \boldsymbol{\beta}\right)} h_{0}\left(t_{(i)}\right) \sum_{l \in R\left(t_{(i)}\right)} \exp \left(\mathbf{x}_{l}^{\prime} \boldsymbol{\beta}\right) \prod_{i=1}^{n} S_{0}\left(t_{i}\right)^{\exp \left(\mathbf{x}_{\mathbf{i}}^{\prime} \boldsymbol{\beta}\right)}
$$

wobei $S_{0}(t)=\exp \left(-\int_{0}^{t} h_{0}(u) d u\right)$. Cox (1972) schlug vor, den ersten Faktor

$$
P L(\boldsymbol{\beta})=\prod_{i=1}^{k} \frac{\exp \left(\mathbf{x}_{(i)}^{\prime} \boldsymbol{\beta}\right)}{\sum_{l \in R\left(t_{(i)}\right)} \exp \left(\mathbf{x}_{l}^{\prime} \boldsymbol{\beta}\right)}
$$

wie eine gewöhnliche Likelihoodfunktion zu behandeln und ihn zur Schätzung von $\boldsymbol{\beta}$ zu maximieren. Da der zweite Faktor auf der rechten Seite von (6.2), der ebenfalls die zu schätzenden Parameter $\boldsymbol{\beta}$ enthält, zum Schätzen der Parameter weggelassen wird, nennt man die Funktion (6.3) partielle Likelihood-Funktion. 
Die Information, die durch Weglassen des rechten Faktors verloren geht, kann sich insbesondere bei kleinen Stichprobenumfängen auf die Güte der Schätzung auswirken. Ein weiterer Informationsverlust kann durch die Einschränkung auftreten, dass bei der partiellen Likelihood-Methode nicht die genauen Ereigniszeiten sondern nur ihre Rangordnung berücksichtigt wird. Mit Hilfe der Theorie der Zählprozesse konnte gezeigt werden, dass partielle Likelihood-Schätzer konsistent und asymptotisch normal aber nicht notwendigerweise asymptotisch effizient (wie ML-Schätzer) sind. Der Ausdruck

$$
\frac{h\left(t_{(i)} \mid \mathbf{x}_{(i)}\right)}{\sum_{l \in R\left(t_{(i)}\right)} h\left(t_{(i)} \mid \mathbf{x}_{l}\right)}=\frac{\exp \left(\mathbf{x}_{(i)}^{\prime} \boldsymbol{\beta}\right)}{\sum_{l \in R\left(t_{(i)}\right)} \exp \left(\mathbf{x}_{l}^{\prime} \boldsymbol{\beta}\right)}
$$

kann interpretiert werden als die bedingte Wahrscheinlichkeit, dass zum Zeitpunkt $t_{(i)}$ gerade für das Individuum mit Kovariablenvektor $\mathbf{x}_{(i)}$ aus der Risikomenge $R\left(t_{(i)}\right)$ ein Ereignis eintrifft, unter der Voraussetzung, dass zum Zeitpunkt $t_{(i)}$ (genau) ein Ereignis stattfindet. Das Produkt über alle $k$ Ereigniszeitpunkte ergibt dann die partielle Likelihoodfunktion (6.3).

Die Konstruktion der Teststatistiken zur Überprüfung der Hypothesen auf Signifikanz einzelner oder mehrerer Variablen erfolgt analog zu Abschnitt 5.1, nur wird statt der (logarithmierten) Likelihoodfunktion (5.7) die partielle (logarithmierte) Likelihoodfunktion (6.3) verwendet. Die dafür notwendigen Voraussetzungen, dass die partiellen Likelihood-Schätzer die gleichen asymptotischen Eigenschaften wie die ML-Schätzer besitzen, wurden erst mit Hilfe der Theorie der Zählprozesse bewiesen; siehe Andersen et al. (1993) oder Fleming und Harrington (1991).

Die partielle Likelihoodfunktion (6.3) ist nur für Ereignisdaten valid, bei denen nicht mehr als ein Ereignis zu einem Zeitpunkt $t$ auftritt. Weniger präzise Messungen führen häufig zu Datensätzen, die mehrere Fälle enthalten, die ein Ereignis zum selben (beliebigen) Zeitpunkt $t$ erleben. Diese Fälle nennt man Bindungen (engl. ties). Bei Vorhandensein von Bindungen muss die partielle Likelihood-Funktion (6.3) modifiziert werden. Breslow (1974) schlägt vor, folgende approximative partielle Likelihood-Funktion zu maximieren, falls mehrere Ereignisse auf einen Zeitpunkt fallen:

$$
P L_{B}=\prod_{i=1}^{k} \frac{\exp \left(\sum_{j \in D\left(t_{(i)}\right)} \mathbf{x}_{j}^{\prime} \boldsymbol{\beta}\right)}{\left[\sum_{j \in R\left(t_{(i)}\right)} \exp \left(\mathbf{x}_{j}^{\prime} \boldsymbol{\beta}\right)\right]^{d_{i}}}
$$

Dabei ist $d_{i}$ die Anzahl der Individuen mit Ereignis zum Zeitpunkt $t_{(i)}$, und $D\left(t_{(i)}\right)$ ist die Menge der Individuen, die zum Zeitpunkt $t_{(i)}$ ein Ereignis erlebt 
haben. Ist die Anzahl gleicher Ereigniszeiten groß, so ist die Approximation von Breslow weniger geeignet. Eine etwas bessere Approximation der exakten Likelihood-Funktion wurde von Efron (1977) vorgeschlagen:

$$
P L_{E}=\prod_{i=1}^{k} \frac{\exp \left(\sum_{j \in D\left(t_{(i)}\right)} \mathbf{x}_{j}^{\prime} \boldsymbol{\beta}\right)}{\prod_{l=1}^{d_{i}}\left[\sum_{j \in R\left(t_{(i)}\right)} \exp \left(\mathbf{x}_{j}^{\prime} \boldsymbol{\beta}\right)-\frac{l-1}{d_{i}} \sum_{j \in D\left(t_{(i)}\right)} \exp \left(\mathbf{x}_{j}^{\prime} \boldsymbol{\beta}\right)\right]}
$$

In den gängigen Software-Produkten sind sowohl der Breslow- als auch der Efron-Schätzer implementiert.

Die Konstruktion einer exakten partiellen Likelihoodfunktion geht davon aus, dass gleiche Ereigniszeiten durch unpräzise Messungen zustande kommen, und dass es somit in Wirklichkeit eine Ordnung dieser (gleichen) Ereigniszeiten gibt. Da diese Ordnung nicht bekannt ist, müssen alle Möglichkeiten einer Ordnung unter den gleichen Ereigniszeiten zur Konstruktion einer exakten partiellen Likelihood-Funktion betrachtet werden, das sind $d_{i}$ ! Möglichkeiten zum Zeitpunkt $t_{(i)}$. Details zur Konstruktion gibt Allison (1998). Ist die Anzahl der Ereignisse an einem Zeitpunkt sehr groß, so ist meist ein diskretes Ereignisdatenmodell geeigneter. Diskrete Ereignisdatenmodelle werden in Kapitel 8 behandelt.

Obwohl die Basis-Hazardrate im Cox-Modell nicht näher spezifiziert wird, ist es möglich, basierend auf den ermittelten Parameterschätzern $\hat{\boldsymbol{\beta}}$ des Cox-Modells, einen Schätzer

$$
\hat{S}(t \mid \mathbf{x})=\hat{S}_{0}(t)^{\exp \left(\mathbf{x}^{\prime} \hat{\boldsymbol{\beta}}\right)}
$$

für die Überlebensfunktion zu ermitteln. Die Basis-Überlebensfunktion $S_{0}(t)$ kann mit Hilfe einer nicht-parametrischen Likelihood-Methode geschätzt werden. Klein und Moeschberger (1997) behandeln die von Breslow (1974) und von Kalbfleisch und Prentice (1973) vorgeschlagenen Schätzer für die kumulative Basis-Hazardrate $H_{0}(t)$, die über $\hat{S}_{0}(t)=\exp \left[-\hat{H}_{0}(t)\right]$ eine Schätzung der Basis-Überlebensfunktion ermöglichen. Die geschätzte Überlebensfunktion $\hat{S}(t \mid \mathbf{x})$ wird für verschiedene Methoden zur Überprüfung des Cox-Modells benötigt; siehe Abschnitt 6.5. Die beiden Schätzer sind auch in der kommerziellen Statistik-Software implementiert. 


\subsection{Zeitabhängige Kovariablen}

Wie schon erwähnt ist die Möglichkeit, zeitabhängige Variablen in das Modell zu integrieren, eine besondere Stärke der Ereignisdatenanalyse. Im Falle von Studienverlaufsanalysen ist anzunehmen, dass der Beginn einer Erwerbstätigkeit bzw. ein Wechsel des Berufsstatus von teilzeit beschäftigt auf voll beschäftigt, also eine Änderung der Variablen Berufstätigkeit während des Studiums, oder etwa die Aufnahme eines zweiten, parallel geführten Studiums die Chancen auf einen erfolgreichen Abschluss verringert. Die Variablen Berufstätigkeit bzw. Doppelstudium müssten realitätsgerecht als zeitveränderliche Variablen in das Modell aufgenommen werden. Ein weiteres Beispiel ist die Verantwortung für ein Kind, die in manchen Fällen während des Studiums übernommen wird.

Das Einbeziehen zeitvariierender Merkmale als zeitkonstante Kovariablen kann zu falschen kausalen Schlüssen führen. In einer Studienverlaufsanalyse soll etwa untersucht werden, ob die Betreuung eines Kindes die Studiendauer der/des Studierenden verlängert. Wird das dichotome Merkmal "Betreuung von Kindern" als zeitkonstante Variable in das Modell integriert, so gibt die Variable lediglich an, ob der/die Studierende während der Studienzeit ein Kind betreut hat oder nicht. Da sich mit der Dauer des Studiums die Chance erhöht, dass ein Kind zu betreuen ist, kann die Einbeziehung dieser Variablen als zeitkonstante Variable zu dem (zumindest teilweise) falschen Schluss führen, dass die Betreuung eines Kindes die Studiendauer verlängert. Wird die Variable jedoch als zeitveränderlich in das Modell einbezogen, so wird der Wert der Variablen erst ab dem Beginn der Kinderbetreuung auf den Wert Eins gesetzt und somit die Gefahr eines falschen (stark verzerrten) kausalen Schlusses vermieden.

Yamaguchi (1991) führt ein ähnliches Beispiel aus der Sozialforschung an. Wird die dichotome Variable "kinderlose Ehe" als zeitkonstantes Merkmal in das Modell einbezogen, so ist der verringernde Effekt eines Kindes auf das Scheidungsrisiko (zumindest teilweise) auf die Tatsache zurückzuführen, dass die "Chance" auf Kinder mit der Dauer der Ehe zunimmt.

Allison (1998) gibt ein konkretes Beispiel aus dem medizinischen Bereich. In einer Studie soll untersucht werden, ob eine Herztransplantation das Sterberisiko von Herzpatienten verringert. Die dichotome Variable "Herztransplantation" wird als zeitkonstante Variable in das Modell integriert. Die Variable gibt in diesem Fall an, ob der Patient bis zum Ende der Beobachtungsperiode eine Herztransplantation gehabt hat oder nicht. In diesem Modell hat die Variable Herztransplantation einen signifikant verringernden Einfluss auf das Sterberisiko. Wird die Variable jedoch als zeitveränderliche Variable in das 
Modell einbezogen, das heißt, der Wert der Variablen wird erst zum Zeitpunkt der Transplantation auf den Wert Eins gesetzt, so zeigt sich kein signifikanter Einfluss der Variablen. Der signifikante Effekt der zeitkonstant modellierten Variablen lässt sich dadurch erklären, dass jene Patienten, die länger leben, größere Chancen auf eine Herztransplantation haben als Patienten, die bald nach Eintritt in die Studie sterben.

Wie im Abschnitt 5.4 erwähnt, können zeitveränderliche Variablen relativ problemlos in das stückweise konstante Hazards-Modell integriert werden. Auch das Cox-Modell erlaubt - auf technisch einfache Weise - die Einbeziehung qualitativ und quantitativ zeitveränderlicher Variablen und die Schätzung der Parameter; diese Möglichkeiten werden auch von der gängigen Statistik-Software unterstützt. Die Einbeziehung zeitveränderlicher Variablen in parametrische Regressionsmodelle und die Schätzung der Parameter ist mit größeren Schwierigkeiten verbunden und wird auch nicht von der gängigen Statistik-Software unterstützt. Aus diesen Gründen werden zeitveränderliche Variablen meist im Zusammenhang mit Cox-Modellen diskutiert.

\section{Kategorisierung von zeitabhängigen Variablen}

Im Allgemeinen werden in der Literatur - meist im Zusammenhang mit der kausalen Interpretation von zeitveränderlichen Variablen und dem darin inkludierten Problem der wechselseitigen Beeinflussung (engl. reverse causation) exogene und endogene zeitabhängige Variablen unterschieden.

Eine Kovariable ist (in Anlehnung an Lancaster (1990)) exogen, falls der Verlauf des Kovariablenprozesses unabhängig von Änderungen im untersuchten Prozess ist, jedoch selbst die zu untersuchende Zeitdauer bis zum Ereignis beeinflussen kann. Dazu gehören folgende in Kalbfleisch und Prentice (1980) als extern bezeichnete Kovariablen:

- zeitkonstante Kovariablen (Geschlecht, Nationalität, Schultyp, Rasse)

- definierte (zeitabhängige) Kovariablen; die Abhängigkeit von der Zeit kann in einer vorher festgelegten funktionalen Form beschrieben werden. Dazu gehören zum Beispiel

- das Alter oder die Verweildauer in einem bestimmten Zustand; bei der Analyse der gesamten Studiendauer von Studierenden, die bereits den ersten Abschnitt beendet haben, ist die "Verweildauer" im zweiten Abschnitt eine definierte zeitabhängige Kovariable 
- Interaktionsvariablen der Zeit und einer zeitunabhängigen Kovariablen (beispielsweise $g(t) \times$ Schultyp), das sind Variablen, die erlauben, dass sich die Effekte bestimmter Kovariablen mit der Zeit ändern; die Einbeziehung solcher Variablen wird häufig zur Überprüfung der Proportionalitätsannahme der zeitunabhängigen Kovariablen verwendet; siehe Abschnitt 6.5

- Hilfsvariablen, das sind Realisierungen eines stochastischen Prozesses, die unabhängig (extern) vom untersuchten Prozess sind (etwa Umwelt- und Wirtschaftsfaktoren); die Einführung von Studiengebühren in einer Analyse der Studiendauer oder die Arbeitslosenrate einer Region in einer Arbeitslosenstudie sind zeitabhängige Kovariablen, die die individuellen Hazardraten beeinflussen können, selbst jedoch unabhängig vom Verlauf des untersuchten Prozesses sind.

Endogene (zeitabhängige) Kovariablen beschreiben einen stochastischen Prozess, dessen Verlauf vom Verlauf (bzw. der Hazardrate) des untersuchten Prozesses abhängt; der Kovariablen- und abhängige Prozess beeinflussen einander somit wechselseitig. Dadurch treten auch sogenannte feedback-Effekte auf, die durch den Effekt des abhängigen Prozesses auf den Kovariablenprozess, der wiederum den anhängigen Prozess beeinflusst, auftreten. Da diese feedbackEffekte von den "echten" Effekten der endogenen Kovariablen (zumeist) nicht getrennt werden können, wird die Interpretation der kausalen Effekte von endogenen Kovariablen in der (vor allem sozialwissenschaftlichen) Literatur als problematisch angesehen. Exogene Variablen sind von diesem Problem nicht betroffen.

$\mathrm{Zu}$ den endogenen Variablen gehören die von Kalbfleisch und Prentice (1980) als intern bezeichneten Variablen, das sind zeitveränderliche Variablen, die nur solange definiert sind, solange das interessierende Ereignis nicht eingetroffen ist. Zum Beispiel kann das Gehalt in einer Studie des Arbeitslosenrisikos nur solange gemessen werden, solange das Individuum eine Arbeit hat. Weitere Beispiele für endogene Kovariablen sind

- die Erwerbstätigkeit oder eine zweite, parallel laufende Ausbildung in Studienverlaufsanalysen; es ist zu etwa vermuten, dass Misserfolg im Hauptstudium die "Chancen" auf den Beginn einer Erwerbstätigkeit oder eines zweiten Studiums erhöhen, und die Erwerbstätigkeit oder die zweite Ausbildung selbst wieder die "Chancen" auf einen erfolgreichen Studienabschluss verringern.

- der Status der Erwerbstätigkeit in einer Analyse der Dauer bis zur Scheidung der ersten Ehe; aus empirischen Studien ist bekannt, dass der Familienstand 
(verheiratet oder geschieden), also der Zustand des abhängigen Prozesses, den Status der Erwerbstätigkeit, also den Kovariablenpfad, beeinflusst.

Wechselseitige Beeinflussung kann zu falschen kausalen Schlüssen führen, falls die Ereigniszeiten und die Änderungszeitpunkte der endogenen Kovariablen nicht exakt gemessen werden. In einer Analyse der Rückfälligkeit von Straftätern (von Allison (1998)) wurde auch die zeitveränderliche Variable Erwerbstätigkeit (in den beiden Ausprägungen voll beschäftigt oder nicht) in das Modell einbezogen. Sowohl der abhängige Prozess als auch der Kovariablenprozess wurden in Wochen gemessen. Falls nun jemand am Anfang einer Woche wieder inhaftiert worden ist, bekommt die Variable Berufstätigkeit für diese Woche (also für den Zeitpunkt der Inhaftierung) den Wert "nicht voll berufstätig" auch wenn der Verhaftete zum Zeitpunkt der Verhaftung voll berufstätig war. Der Effekt, dass ehemalige Häftlinge, die nicht voll berufstätig sind, gefährdeter für einen Rückfall sind als voll beschäftigte Straftäter, wird somit verstärkt. Eine Möglichkeit, diesem Problem zu begegnen, ist die Einbeziehung der Variablen Berufstätigkeit in Abhängigkeit von der Vorwoche. In diesem Fall wird analysiert, ob Personen, die in der Vorwoche $t-1$ nicht voll berufstätig waren, eine höhere Rückfälligkeitsrate in der Woche $t$ haben als jene, die voll berufstätig waren.

In Anwendungsbeispielen werden endogene Kovariablen genauso wie exogene Variablen in das Ereignisdatenmodell integriert und mit der (partiellen) Likelihood-Methode geschätzt; der Effekt der endogenen Variablen wird in gleicher Weise wie der Effekt von exogenen Variablen analysiert - meist mit dem Hinweis, dass bei der kausalen Interpretation der endogenen Variablen Vorsicht geboten ist.

Im Zusammenhang mit der Einbeziehung von (endogenen) zeitveränderlichen Variablen wird auch häufig das Problem der unbeobachteten Heterogenität diskutiert, dem in der Ereignisdatenanalyse mehr Bedeutung als in der linearen Regressionsanalyse zukommt. Von unbeobachteter Heterogenität spricht man, falls neben den in das Modell aufgenommenen Variablen weitere Merkmale, die nicht erhoben worden oder nicht bekannt sind, den abhängigen Prozess beeinflussen. Wangler (1997) befasst sich in ihrer Arbeit schwerpunktmäßig mit diesem Thema. Die Konsequenzen von unbeobachteter Heterogenität auf die Abhängigkeit der Hazardfunktion von der Zeit - die Nichtberücksichtigung der Heterogenität führt entweder zu einer Überschätzung des negativen Verweildauereffektes oder zu einer Unterschätzung des positiven Verweildauereffektes - und auf die (kausale) Interpretation des Effektes der einbezogenen Variablen diskutieren auch Vermunt (1997), Yamaguchi (1991) und Blossfeld und Rohwer (1995) ausführlich. 
Die Kategorisierung von zeitveränderlichen Kovariablen behandeln im Detail Kalbfleisch und Prentice (1980), Lancaster (1990), Yamaguchi (1991), Blossfeld und Rohwer (1995) und Vermunt (1997).

\section{Zeitabhängige Variablen im Cox-Modell}

Die Einbeziehung von zeitveränderlichen Kovariablen in das Cox-Modell erfolgt in der Form

$$
\begin{aligned}
h[t \mid \mathbf{x}(t)] & =h_{0}(t) \exp \left[\sum_{j=1}^{p_{1}} \beta_{j} x_{j}+\sum_{j=p_{1}+1}^{p} \beta_{j} x_{j}(t)\right] \\
& =h_{0}(t) \exp \left(\mathbf{x}^{\prime}(t) \boldsymbol{\beta}\right)
\end{aligned}
$$

Die Gleichung (6.4) ist die verallgemeinerte Darstellung des Cox-Modells, denn eine zeitkonstante Variable kann betrachtet werden als

$$
x(t)=x(t=0)=x .
$$

Im Modell (6.4) hängt die Hazardrate zum Zeitpunkt $t$ von den zeitunabhängigen Kovariablen $x_{1}, \ldots, x_{p_{1}}$ und von den Werten der zeitabhängigen Variablen $x_{p_{1}+1}(t)$, $\ldots, x_{p}(t)$ zum Zeitpunkt $t$ ab.

Im Zusammenhang mit dem Problem der wechselseitigen Beeinflussung wurde bereits diskutiert, dass es - je nach Problemstellung - manchmal sinnvoll ist, endogene zeitabhängige Variablen in Abhängigkeit von $t-1$ in das Modell einzubeziehen. In praktischen Anwendungen gibt es verschiedene Möglichkeiten, wie endogene zeitabhängige Variablen in ein Modell einbezogen werden können. Wollen wir beispielsweise die Studiendauer in Abhängigkeit der Berufstätigkeit modellieren, so kann die als durchschnittliches Wochenstundenausmaß je Monat gemessene Variable Berufstätigkeit zum Zeitpunkt $t$ etwa wie folgt definiert werden: $x(t)$ gibt das durchschnittliches Wochenstundenausmaß

- im laufenden Monat $t$

- im vorangegangenen Monat $t-1$

- in den letzten zwei Semestern

- seit Beginn des Studiums 
an. Ganz allgemein wollen wir deshalb in der vorliegenden Arbeit den Ausdruck $x(t)$ als jenen Wert der in das Modell einbezogenen zeitabhängigen Kovariablen verstehen, mit dem diese Variable zum Zeitpunkt $t$ in die ML-Schätzung eingeht; vgl. Abschnitt 12.3 und Allison (1998).

Im verallgemeinerten Cox-Modell sind die Hazardraten zweier Individuen $k$ und $m$ nicht mehr proportional:

$$
\begin{aligned}
H R(t) & =\frac{h\left[t, \mathbf{x}_{k}(t)\right]}{h\left[t, \mathbf{x}_{m}(t)\right]} \\
& =\exp \left[\sum_{j=1}^{p_{1}} \beta_{j}\left(x_{j k}-x_{j m}\right)+\sum_{j=p_{1}+1}^{p} \beta_{j}\left[x_{j k}(t)-x_{j m}(t)\right]\right]
\end{aligned}
$$

Die Verallgemeinerung der partiellen Likelihood-Funktion ist

$$
P L(\boldsymbol{\beta})=\prod_{i=1}^{k} \frac{\exp \left(\mathbf{x}_{(i)}^{\prime}\left(t_{(i)}\right) \boldsymbol{\beta}\right)}{\sum_{l \in R\left(t_{(i)}\right)} \exp \left(\mathbf{x}_{l}^{\prime}\left(t_{(i)}\right) \boldsymbol{\beta}\right)} .
$$

Aus (6.5) ist zu erkennen, warum die Einbeziehung zeitveränderlicher Variablen in das Cox-Modell (technisch) einfach zu handhaben ist; zu jedem Ereigniszeitpunkt $t_{(i)}$ wird für jedes Individuum aus der Risikomenge $R\left(t_{(i)}\right)$ der aktuelle Wert der zeitveränderlichen Kovariablen für die partielle LikelihoodSchätzung verwendet. Allerdings müssen zu jedem Ereigniszeitpunkt für jedes Individuum aus der Risikomenge die Werte der zeitveränderlichen Variablen überprüft werden; die partielle Likelihood-Schätzung unter Berücksichtigung von zeitabhängigen Variablen ist daher sehr rechenintensiv.

Die Schätzung der Parameter auf Basis von (6.5) erfordert eine genaue Aufzeichnung der Kovariablenpfade. Zu jedem Ereigniszeitpunkt $t_{(i)}$ müssen für alle Individuen aus $R\left(t_{(i)}\right)$ die Werte der Kovariablen bekannt sein. Die zeitabhängige Kovariable muss daher in kleineren oder gleichen Einheiten, zum Beispiel in Tagen oder Wochen, wie der interessierende Prozess gemessen werden. Oft werden zeitveränderliche Variablen in regelmäßigen Abständen gemessen, beispielsweise monatliche Messung des Blutdrucks bei Patienten. Wird der abhängige Prozess in kleineren Einheiten modelliert, so ist der exakte Wert der zeitveränderlichen Kovariablen für die Ereigniszeitpunkte nicht bekannt. Beispielsweise erscheint es nicht sinnvoll, die Berufsintensität von Studierenden (in Wochenstunden) retrospektiv in Monaten zu erheben, da sich die Befragten mit Sicherheit nicht mehr an die genauen Daten erinnern werden. Die Berufstätigkeit muss daher semesterweise (oder jährlich) erhoben werden, während die Dauer bis zum Studienende hingegen in Monaten modelliert werden kann. In diesem Fall werden Approximationen für den Wert der Kovaria- 
blen zum Zeitpunkt $t_{(i)}$ eingesetzt. Näheres zu diesem Problem findet sich im Abschnitt 12.3 bzw. in Allison (1998)

\section{Episodensplitting zur Einbeziehung zeitabhängiger Va- riablen in parametrische Regressionsmodelle}

Während die partielle Likelihood-Schätzung relativ einfach für die Einbeziehung zeitveränderlicher Variablen modifiziert werden kann und dies auch von der gängigen Statistik-Software unterstützt wird, ist die Anpassung der Maximum-Likelihood-Methode zur Schätzung von parametrischen Regressionsmodellen mit zeitveränderlichen Variablen nicht so problemlos.

Diskrete zeitveränderliche Variablen folgen im Prozessverlauf der Gestalt einer Treppenfunktion und sind stückweise konstant. Wird ein Individuum $i$ von $t_{0}=$ 0 bis $t_{i}$ beobachtet, werden mit $0<t_{1_{i}}<\ldots<t_{s_{i}}$ die $s_{i}$ Änderungszeitpunkte einer diskret zeitveränderlichen Variablen im Intervall $\left[0, t_{i}\right)$ bezeichnet und sei $t_{s_{i}+1}=t_{i}$ der Ereignis- bzw. Zensierungszeitpunkt des $i$-ten Individuums, dann kann der Beitrag des $i$-ten Individuums zur Likelihoodfunktion geschrieben werden als:

$$
L_{i}(\beta)=\prod_{r=1}^{s_{i}+1}\left\{h\left[t_{r_{i}} \mid \mathbf{x}_{i}\left(t_{r_{i}-1}\right)\right]\right\}^{\delta_{r_{i}}} S\left[t_{r_{i}} \mid t_{r_{i}-1} ; \mathbf{x}_{i}\left(t_{r_{i}-1}\right)\right],
$$

wobei

$$
\delta_{r_{i}}= \begin{cases}1 & r_{i}=s_{i}+1 \text { und das } i \text {-te Individuum ist nicht zensiert } \\ 0 & r_{i}=s_{i}+1 \text { und das } i \text {-te Individuum ist rechts-zensiert } \\ 0 & r_{i}=1, \ldots, s_{i}\end{cases}
$$

und

$$
S\left[t_{r} \mid t_{r-1} ; \mathbf{x}\left(t_{r-1}\right)\right]=\exp \left(-\int_{t_{r-1}}^{t_{r}} h\left[u \mid \mathbf{x}\left(t_{r-1}\right)\right] d u\right) .
$$

Zur Schätzung der unbekannten Parameter mit der ML-Methode wird die Beobachtungsperiode des Individuums $i$ somit in $s_{i}+1$ Subepisoden aufgeteilt. Jede Subepisode wird dann in der Schätzung als eigenständige (links abgeschnittene) Beobachtung mit zeitkonstanten Kovariablen behandelt und enthält

1. die Ausprägungen der Kovariablen zu Beginn der Subepisode,

2. die Zeitpunkte von Beginn und Ende der Subepisode und 
3. den Zensierungsindikator, ob die Subepisode mit einem Ereignis geendet hat oder nicht.

Beispielsweise kann in einer Studienverlaufsanalyse berücksichtigt werden, ob die Studierenden Kinder versorgen oder nicht. Wird dann eine Studentin während ihrer Studienzeit Mutter (oder ein Student Vater), so wird ihre Studienzeit in zwei Subepisoden unterteilt - in die kinderlose Studienzeit bis zur Geburt und danach in die Periode bis zum Ende bzw. Abbruch des Studiums oder bis zum Ende des Beobachtungszeitraums, falls das Studium dann noch im Gange ist. Die Risikoperiode der zweiten Subepisode beginnt mit der Geburt des Kindes, und die Beobachtung, die die zweite Subepisode beschreibt, ist daher links abgeschnitten. Vermunt (1997) und Blossfeld und Rohwer (1995) zeigen, dass im Falle von semiparametrischen Cox-Modellen die Maximierung der Likelihoodfunktion (6.6) der Maximierung der verallgemeinerten partiellen Likelihood-Funktion (6.5) entspricht.

Bei stetigen zeitveränderlichen Variablen, die keine bestimmte vorgegebene Funktion der Verweildauer sind, besteht diese einfache Möglichkeit der Einbeziehung in das parametrische Modell nicht. Eine mögliche Lösung besteht darin, den Verlauf der stetigen zeitveränderlichen Variablen zu approximieren, indem man sie über festgelegte Subepisoden als stückweise konstant betrachtet und ihre Werte zu Beginn jeder Subepisode aktualisiert. Die Vorgehensweise zur Schätzung der unbekannten Parameter erfolgt dann wie bei diskreten zeitveränderlichen Variablen.

Mit der Einbeziehung von zeitveränderlichen Variablen in parametrische Regressionsmodelle setzen sich beispielsweise Blossfeld et al. (1986), Blossfeld und Rohwer (1995) und Petersen (1986) auseinander. Die Maximierung von (6.6) ist mit der gängigen Statistik-Software nicht möglich; jedoch unterstützt das Programm TDA dieses Methode für zahlreiche parametrische Regressionsmodelle.

\subsection{Das stratifizierte Cox-Modell}

Ist bei der Überprüfung der Proportionalitätsannahme (siehe Abschnitt 6.5) festgestellt worden, dass diese Annahme für eine der einbezogenen zeitunabhängigen Kovariablen verletzt ist, dann ist es möglich, die Daten nach dieser Variablen zu schichten und für jede Schicht ein proportionales Hazards-Modell in Abhängigkeit der anderen Kovariablen aufzustellen:

$$
h_{g}[t \mid \mathbf{x}(t)]=h_{0 g}(t) \exp \left(\mathbf{x}^{\prime}(t) \boldsymbol{\beta}\right), \quad g=1, \ldots, s,
$$


wobei $s$ die Anzahl der Kategorien der Schichtungsvariablen ist. Kontinuierlich skalierte Variablen, die die Proportionalitätsannahme nicht erfüllen, müssen erst gruppiert werden, um das stratifizierte Modell anwenden zu können. Da die Schichtungsvariable nicht im Modell inkludiert ist, erhält man bei diesem Modell keine Aussagen über den Effekt dieser Variablen. Daher eignet sich das stratifizierte Modell nur dann, falls Aussagen über den Effekt der Schichtungsvariablen nicht von Interesse sind. Die Hazardfunktionen der einzelnen Schichten im Modell (6.7) unterscheiden sich in den Basis-Hazardraten, jedoch nicht in den Parameterschätzern $\boldsymbol{\beta}$. Es wird also im stratifizierten Cox-Modell (6.7) angenommen, dass die Effekte der in das Modell einbezogenen Variablen in allen Schichten gleich sind, also nicht von der Schicht abhängen. Das ist gleichbedeutend mit der Annahme, dass es keine Interaktionseffekte der einbezogenen Kovariablen mit der Schichtungsvariable gibt.

Die Schätzung der Parameter erfolgt über Maximierung der partiellen Likelihood-Funktion, die durch Multiplizieren der partiellen Likelihoodfunktionen jeder einzelnen Schicht erhalten wird:

$$
P L_{S}(\boldsymbol{\beta})=\prod_{g=1}^{s} P L_{g}(\boldsymbol{\beta})
$$

Auch für den Fall, dass mehrere zeitunabhängige Variablen die Proportionalitätsannahme nicht erfüllen, kann das stratifizierte Cox-Modell verwendet werden, indem alle möglichen Kombinationen zwischen den Kategorien der betreffenden Variablen gebildet werden. Im Falle von $p$ Kovariablen mit jeweils $g_{j}$ Kategorien, erhält man somit $s=g_{1} \times g_{2} \times \cdots \times g_{p}$ Schichten.

Um zu testen, ob die Effekte der einbezogenen Variablen in den Schichten gleich sind, dass also keine Interaktionseffekte zwischen den Schichten und den einbezogenen Variablen auftreten, wird ein Modell gebildet, dass von unterschiedlichen Parameterschätzern in den Schichten ausgeht:

$$
h_{g}[t \mid \mathbf{x}(t)]=h_{0 g}(t) \exp \left(\mathbf{x}^{\prime}(t) \boldsymbol{\beta}_{g}\right) \quad g=1, \ldots, s,
$$

wobei $s=g_{1} \times g_{2} \times \cdots \times g_{p}$ gilt. Es wird also für jede Schicht separat ein eigenes Cox-Modell geschätzt. Das Modell (6.8) ist äquivalent zu einem Modell, das in das stratifizierte Cox-Modell (6.7) alle möglichen Interaktionsvariablen zwischen den Schichten und den einbezogenen Kovariablen aufnimmt. Für den einfachsten Fall einer dichotomen Schichtungsvariablen (beispielsweise das Geschlecht) und einer in das Modell einbezogenen zeitunabhängigen Kovariable $x$, die die Proportionalitätsannahme erfüllt, erhält man die stratifizierten Hazardraten:

$$
h_{g}(t \mid x)=h_{0 g} \exp \left(x \beta_{g}\right)=h_{0 g} \exp \left(x \beta_{1}^{*}+(\operatorname{sex} \times x) \beta_{2}^{*}\right),
$$


wobei $g=1,2$ und sex $=1$ für weibliche und sex $=0$ für männliche Fälle gilt.

Mit Hilfe des Likelihood-Quotiententests kann beispielsweise die globale Hypothese getestet werden, dass die Parameterschätzer jeweils in allen Schichten gleich sind: $H_{0}: \beta_{j 1}=\ldots=\beta_{j s}$ für alle $j=1, \ldots, p$. Zur Durchführung des Tests werden die entsprechenden logarithmierten partiellen Likelihoodfunktionen $\ln P L()=.P l($.$) für die beiden Modelle (6.7) und (6.8) gebildet und dann$ die Likelihood-Quotientenstatistik

$$
G=2\left[\sum_{g=1}^{s} P l_{g}\left(\hat{\boldsymbol{\beta}}_{g}\right)-P l_{S}(\hat{\boldsymbol{\beta}})\right]
$$

ermittelt. Unter $H_{0}$ ist die Teststatistik asymptotisch $\chi^{2}$ mit $(g-1) p$ Freiheitsgraden verteilt.

Analog zu Abschnitt 5.2 können auch Hypothesen über die Gleichheit einzelner Regressionskoeffizienten - unter Berücksichtigung der anderen in das Modell einbezogenen Variablen - getestet werden: $H_{0}: \beta_{j 1}=\ldots=\beta_{j s}$.

Das stratifizierte Cox-Modell wird ausführlich in Kleinbaum (1996) diskutiert sowie in Allison (1998), Blossfeld und Rohwer (1995), Hosmer und Lemeshow (1999) und Klein und Moeschberger (1997). Die in diesem Abschnitt diskutierten Ansätze werden von der gängigen Statistik-Software unterstützt.

\subsection{Modellüberprüfungen}

In diesem Abschnitt werden Methoden zur Überprüfung der Adäquatheit des angepassten Cox-Modells besprochen. Die allgemeine Anpassungsgüte des Modells, die funktionale Form der einbezogenen Kovariablen, die Proportionalitätsannahme und mögliche Ausreißer sind Thema dieses Abschnitts. In Ereignisdatenmodellen können Residuen nicht so einfach wie in linearen Regressionsmodellen definiert werden. In der Literatur werden verschiedene Konzepte von Residuen für das Cox-Modell definiert, die je nach dem Aspekt des Modells, der überprüft werden soll, eingesetzt werden. Einige der Residuen und ihre Verwendung werden in diesem Abschnitt behandelt.

\section{Allgemeine Anpassung an die Daten - Cox Snell Residu- en}

Im Abschnitt 5.3 haben wir einige Methoden kennengelernt, wie man die Adäquatheit der getroffenen Verteilungsannahmen in einem parametrischen 
Modell überprüfen kann. Cox-Snell Residuen wurden eingeführt, um die Anpassungsgüte des gewählten Modells unter Einbeziehung von Kovariablen zu überprüfen. Diese Residuen können analog für das Cox-Modell (mit zeitunabhängigen Kovariablen) definiert werden:

$$
\hat{e}_{i}=\hat{H}_{0}\left(t_{i}\right) \exp \left(\mathbf{x}_{i}^{\prime} \hat{\boldsymbol{\beta}}\right)=\hat{H}\left(t_{i} \mid \mathbf{x}_{i}\right),
$$

wobei $\hat{H}_{0}(t)$ ein Schätzer der kumulativen Basis-Hazardrate ist; siehe Abschnitt 6.2. Hat das Cox-Modell eine gute Anpassungsgüte, dann sollten sich die $\hat{e}_{i}$ 's annähernd wie eine zensierte Stichprobe von einer Exponentialverteilung mit Parameter $\lambda=1$ verhalten. Ein Steudiagramm von $-\ln (\hat{S}(\hat{e}))$ gegen $\hat{e}$, wobei $\hat{S}(\hat{e})$ eine nicht-parametrische Schätzfunktion (etwa nach der KaplanMeier oder Nelson-Aalen-Methode) der Überlebensfunktion ist, sollte daher annähernd eine Gerade durch den Ursprung mit Anstieg Eins ergeben.

Allison (1998) und Blossfeld et al. (1986) raten von der Verwendung von CoxSnell-Residuen bei Cox-Modellen ab, da Abweichungen von der Exponentialverteilung vor allem bei kleinen Stichproben teilweise auf Unsicherheiten beim Schätzen von $\boldsymbol{\beta}$ und $H_{0}(t)$ zurückzuführen sind. Auch Klein und Moeschberger (1997) bemerken, dass die Ergebnisse des graphischen Residuentests mit Vorsicht $\mathrm{zu}$ interpretieren sind.

\section{Funktionale Form der kontinuierlichen Kovariablen - Martingal-Residuen}

Ein wichtiger Schritt der Modellüberprüfung ist die Überprüfung der Annahme, dass die in das Modell einbezogenen Variablen linear auf die logarithmierte Hazardrate

$$
\ln [h(t \mid \mathbf{x})]=\ln \left[h_{0}(t)\right]+\mathbf{x}_{i}^{\prime} \boldsymbol{\beta}
$$

wirken, und falls nicht, für welche Transformation $f(x)$ der Kovariablen die Linearität des Effektes auf die logarithmierte Hazardrate erreicht werden kann. In Frage kommende Transformationen sind beispielsweise $\ln (x), x^{2}$ sowie die Indikatorfunktion $I_{x \leq c}$, die für $x \leq c$ den Wert Eins und sonst Null annimmt. Untersucht wird also die Beziehung

$$
\ln [h(t \mid \mathbf{x})]=\ln \left[h_{0}(t)\right]+\sum_{j=1}^{k} f\left(x_{j}\right) \beta_{j} .
$$

Eine Möglichkeit, die Form des Effektes einer Kovariablen graphisch zu überprüfen, basiert auf Martingal-Residuen, die (für rechts-zensierte Daten und zeitunabhängige Kovariablen) definiert sind als:

$$
\hat{M}_{i}(\infty)=\delta_{i}-\hat{H}_{0}\left(t_{i}\right) \exp \left(\mathbf{x}_{i}^{\prime} \hat{\boldsymbol{\beta}}\right)=\delta_{i}-\hat{e}_{i}, \quad i=1, \ldots, n
$$


wobei $\delta_{i}$ angibt, ob die $i$-te Beobachtung rechts-zensiert ist oder nicht. Die Residuen haben folgende Eigenschaften:

1. $\sum_{i=1}^{n} \hat{M}_{i}=0$

2. $\operatorname{Cov}\left(\hat{M}_{i}, \hat{M}_{j}\right)=0$ für großes $n$

3. $E\left(\hat{M}_{i}\right)=0$ für großes $n$

Martingal-Residuen können als Differenz zwischen den beobachteten und den - unter Annahme eines Cox-Modells - erwarteten Ereignissen interpretiert werden.

Um die funktionale Form einer Kovariablen zu überprüfen, schlagen Therneau et al. (1990) vor, ein Cox-Modell zu implementieren, das alle Kovariablen ausser die zu untersuchende enthält, wobei angenommen wird, dass die funktionale Form der einbezogenen Kovariablen bekannt ist und die zu überprüfende Variable unabhängig von den einbezogenen Variablen ist. Auf Basis dieses Cox-Modells werden dann die Martingal-Residuen, $\hat{M}_{i}, i=1, \ldots, n$, berechnet. Dann werden die Martingal-Residuen gegen die zu untersuchende Kovariable aufgetragen, und Therneau et al. zeigen, dass die (etwa mit der LOWESSMethode, siehe Cleveland (1979)) geglätteten Residuen annähernd die funktionale Form $f(x)$ angeben. Insbesondere zeigt eine annähernd lineare Kurve, dass die Kovariable nicht transformiert werden muss, und nicht-lineare Kurven können Hinweise geben, welche Transformation passend ist. Werden sowohl die individuellen Residuen als auch die Glättungskurve aufgetragen, erhält man auch Hinweise, welche Beobachtungen die Schätzung der funktionalen Form beeinflussen. Für parametrische Modelle werden die Martingal-Residuen ana$\log$ mit $M_{i}=\delta_{i}-\hat{e}_{i}$ definiert; die Cox-Snell-Residuen $\hat{e}_{i}$ für parametrische Modelle wurden im Abschnitt 5.3 behandelt.

Grambsch et al. (1995) ergänzen diese Überlegungen und schlagen vor, ein CoxModell mit allen Kovariablen zu implementieren und auf Basis dieses Modells die Martingal-Residuen $\hat{M}_{i}$ zu berechnen. Diese Residuen werden verwendet, um die Cox-Snell-Residuen $\hat{e}_{i}=\delta_{i}-\hat{M}_{i}$ zu ermitteln. Sowohl die Werte von $\delta_{i}$ als auch die von $\hat{e}_{i}$ werden dann gegen jene Variable aufgetragen, deren funktionale Form untersucht wird, und die beiden (etwa mit der LOWESSMethode ermittelten) Glättungsfunktionen $\operatorname{smooth}(\delta)$ und $\operatorname{smooth}(\hat{e})$ gebildet. Die funktionale Form der zu überprüfenden Kovariablen $x_{j}$ folgt dann annähernd folgender Beziehung:

$$
f \approx \ln \left(\frac{\operatorname{smooth}(\delta)}{\operatorname{smooth}(\hat{e})}\right)+\hat{\beta}_{j} x_{j}
$$


In Simulationsstudien zeigen die Autoren, dass diese Methode - vor allem im Fall von Korrelationen zwischen den Kovariablen - bessere Diagnosen der funktionalen Form der betrachteten Kovariablen als das eingangs beschriebene Verfahren erlaubt.

In praktischen Beispielen muss auf die Interpretierbarkeit der gewählten funktionalen Form geachtet werden.

\section{Proportionalitätsannahme}

Proportionale Hazards-Modelle verdanken ihren Namen der Eigenschaft, dass im Modell ohne zeitabhängige Kovariablen die Hazardrate des $j$-ten Individuums mit Kovariablenvektor $\mathbf{x}_{j}$ ein Vielfaches von der Hazardrate des $i$-ten Individuums mit Kovariablenvektor $\mathbf{x}_{i}$ ist. Im Abschnitt 6.4 ist das stratifizierte Cox-Modell besprochen worden, das bei Verletzung der Proportionalitätsannahme verwendet werden kann. In diesem Abschnitt werden einige Methoden vorgestellt, mit denen die Proportionalitätsannahme überprüft werden kann.

Eine populäre Methode ist die Einführung von zeitabhängigen Interaktionsvariablen zwischen der zu überprüfenden zeitunabhängigen Kovariablen und der Zeit:

$$
h(t \mid \mathbf{x})=h_{0}(t) \exp \left(\sum_{j=1}^{p}\left[\beta_{j} x_{j}+c_{j} x_{j} g_{j}(t)\right]\right)=h_{0}(t) \exp \left(\sum_{j=1}^{p}\left[\beta_{j}+c_{j} g_{j}(t)\right] \mathbf{x}_{j}\right),
$$

wobei $g_{j}(t)$ geeignete Funktionen in $t$ sind; häufig werden für $g(t)$ die Funktionen $t, \ln (t)$ oder $I_{t \leq t_{0}}$ verwendet. In diesem Modell kann dann (etwa mit der Likelihood-Quotienten-Statistik) getestet werden, ob alle Koeffizienten $c_{j}, j=$ $1, \ldots, p$, gleich Null sind, also die Hypothese $H_{0}: c_{1}=c_{2}=\ldots=c_{p}=0$ gilt und somit ein proportionales Modell vorliegt, oder für jeden einzelnen Koeffizienten die Hypothese $H_{0}: c_{j}=0$ (etwa mit der Wald-Teststatistik) überprüft werden.

Ein Nachteil dieser Methode ist, dass verschiedene $g(t)$ zu unterschiedlichen Ergebnissen führen können. Einen Anhaltspunkt für die Form von $g(t)$ geben die approximierten skalierten Schoenfeld-Residuen, die von Grambsch und Therneau (1994) vorgeschlagen wurden:

$$
\hat{\mathbf{r}}_{i}^{*}=m \widehat{\operatorname{Var}}(\hat{\boldsymbol{\beta}}) \hat{\mathbf{r}}_{i},
$$

wobei $m$ die Anzahl der Ereignisse und $\hat{\mathbf{r}}_{i}=\left(\hat{r}_{i 1}, \hat{r}_{i 2}, \ldots, \hat{r}_{i p}\right)^{\prime}$ der Vektor der (geschätzten) Schoenfeld-Residuen

$$
\hat{r}_{i k}=\delta_{i}\left(x_{i k}-\hat{\bar{x}}_{k}\right)
$$


mit

$$
\hat{\bar{x}}_{k}=\frac{\sum_{j \in R\left(t_{i}\right)} x_{j k} e^{\mathbf{x}_{j}^{\prime} \hat{\boldsymbol{\beta}}}}{\sum_{j \in R\left(t_{i}\right)} e^{\mathbf{x}_{j}^{\prime} \hat{\boldsymbol{\beta}}}}
$$

für das $i$-te Individuum ist; $\hat{r}_{i k}$ ist missing, falls das Individuum zensiert, also $\delta_{i}=0$ ist, $t_{i}$ ist die Ereigniszeit des $i$-ten Individuums, $R\left(t_{i}\right)$ ist die Risikomenge zu diesem Zeitpunkt und $x_{i k}$ ist der Wert der $k$-ten Kovariablen des $i$-ten Individuums; $\hat{\bar{x}}_{k}$ ist ein Schätzer des erwarteten Werts der Kovariablen $x_{k}$ für ein zufällig aus der Risikomenge $R\left(t_{i}\right)$ ausgewähltes Individuum.

Grambsch und Therneau zeigen, dass für die $j$-te Kovariable approximativ

$$
E\left[r_{j}^{*}(t)\right] \approx c_{j} g_{j}(t)
$$

gilt, und ein Plot der geglätteten Schoenfeldresiduen $\hat{r}_{i j}^{*}$ gegen $t$ Hinweise gibt, ob $c_{j}=0$ ist, und falls nicht, welche Form $g_{j}(t)$ besitzt.

Ein weitere populäre, graphische Methode zur Überprüfung der Proportionalitätsannahme basiert auf der folgenden Beziehung zwischen zwei Individuen mit Kovariablenvektoren $\mathbf{x}_{\mathbf{m}}$ und $\mathbf{x}_{\mathbf{k}}$ :

$$
\ln \left[-\ln S\left(t \mid \mathbf{x}_{m}\right)\right]-\ln \left[-\ln S\left(t \mid \mathbf{x}_{k}\right)\right]=\sum_{j=1}^{p} \beta_{j}\left(x_{m j}-x_{k j}\right)
$$

Um die Proportionalitätsannahme für eine Kovariable zu überprüfen, können die Daten beispielsweise nach dieser Variablen geschichtet und für jede Schicht ein proportionales Hazards-Modell in Abhängigkeit von jenen Kovariablen, die die Proportionalitätsannahme erfüllen, geschätzt werden. Zeigt ein Plot, dass die geschichteten logarithmierten Überlebensfunktionen (unter Berücksichtigung der anderen Kovariablen in Form ihrer Mittelwerte $\overline{\mathbf{x}}=1 / n \sum_{i=1}^{n} \mathbf{x}_{i}$ )

$$
\ln \left[-\ln \hat{S}_{j}(t \mid \overline{\mathbf{x}})\right]=\ln \left[-\ln \hat{S}_{0 j}\right]+(\overline{\mathbf{x}} \boldsymbol{\beta}), \quad j=1, \ldots, s,
$$

annähernd parallel sind, dann ist dies ein Hinweis dafür, dass die Proportionalitätsannahme für die betrachtete Variable erfüllt ist.

\section{Ausreißer - Deviance-Residuen}

Deviance-Residuen werden verwendet, um Beobachtungen zu identifizieren, deren Ereigniszeiten vom angepassten Modell nicht gut prognostiziert werden; sie sind definiert als:

$$
D_{i}=\operatorname{sign}\left[\hat{M}_{i}\right]\left\{-2\left[\hat{M}_{i}+\delta_{i} \log \left(\delta_{i}-\hat{M}_{i}\right)\right]\right\}^{1 / 2}
$$


Deviance-Residuen sind (im Gegensatz zu den Martingal-Residuen $\hat{M}_{i}$ ) annähernd symmetrisch um Null verteilt; sie sind negativ für Beobachtungen mit längeren Zeitdauern (bis zum Ereignis) als erwartet und positiv für kürzere Zeitdauern als erwartet. Klein und Moeschberger (1997) schlagen vor, die Residuen $D_{i}$ gegen $\sum_{j=1}^{p} \hat{\beta}_{j} x_{i j}$ aufzutragen. Potentielle Ausreißer haben dann Deviance-Residuen, deren absolute Werte zu groß sind.

Mit Residuen in parametrischen und semiparametrischen Ereignisdatenmodellen und ihrer Anwendung zur Überprüfung verschiedener Aspekte des implementierten Modells setzen sich beispielsweise Collett (1994) sowie Klein und Moeschberger (1997) ausführlich auseinander. Die in diesem Abschnitt angeführten Residuen sind für semiparametrische Modelle etwa in SAS und S-PLUS implementiert und für parametrische Modelle in S-PLUS. 


\section{Kapitel 7}

\section{Mehr-Zustands- und Mehr-Episoden-Modelle}

Die in den bisherigen Kapiteln behandelten Methoden wurden für (kontinuierliche) Modelle mit einem Anfangszustand und einem Endzustand erläutert. In diesem Kapitel werden die wichtigsten Grundbegriffe und Methoden auf MehrZustands- und Mehr-Episoden-Modelle erweitert, die für die Modellierung von Studienverlaufsdaten von Bedeutung sind.

\subsection{Mehr-Zustands-Modelle}

In den vorherigen Kapiteln sind wir davon ausgegangen, dass die Dauer einer Episode durch ein bestimmtes Ereignis beendet wird. Bei Studienverlaufsanalysen kann das Studium in den Zuständen "abgebrochen" und "erfolgreich abgeschlossen" enden. Zwar können die gleichen Kovariablen wie schulische Vorbildung, Familenstatus und Berufstätigkeit die beiden Ereignisse beeinflussen, jedoch sind die beiden Ereignisse unterschiedlich und erfordern daher die Implementierung eines Mehr-Zustands-Modells. In einer Berufsverlaufsstudie können etwa die Determinanten für das Ausscheiden aus der ersten Arbeit von Interesse sein. Das Ausscheiden aus der Arbeit ist das interessierende Ereignis, das die Phase des ersten Jobs beendet. Es ist jedoch zu vermuten, dass unterschiedliche Determinanten dafür verantwortlich sind, ob der Arbeitnehmer freiwillig kündigt oder entlassen wird. Der Berufsausstieg kann somit im Zustand "entlassen" oder "freiwillig ausgeschieden" enden, und zur Analyse von ereignisspezifischen Determinanten ist ein Mehr-Zustands-Modell zu implementieren. 
Mehr-Zustands-Modelle werden in der Literatur in zwei ideal-typischen Situationen unterschieden:

Typ I-Situation: Diese Situation tritt auf, wenn das Eintreffen eines Ereignisses - unabhängig von seinem Typ - von einem kausalen Prozess bestimmt wird, und sobald das Ereignis eingetroffen ist, bestimmt ein zweiter, dem ersten nachfolgender Prozess, welcher Art die Entscheidung sein wird.

Die Typ I-Situation ist etwa bei Kaufentscheidungen valid; im ersten Schritt wird entschieden, ob man sich überhaupt ein Auto kaufen möchte, und in einem weiteren Schritt, nachdem man sich über den Kauf eines Autos sicher ist, wird man sich für eine bestimmte Marke entscheiden.

In diesen Fällen wird im ersten Schritt ein Hazards-Modell - mit den Methoden aus den vorangegangen Kapiteln - implementiert. Im zweiten Schritt werden die bedingten Wahrscheinlichkeiten für eine bestimmte Entscheidung in Form eines Logit- oder Probit-Modells modelliert.

Hachen (1988) erklärt, dass die Modellierung einer Typ I-Situation dann passend ist, wenn zu erwarten ist, dass die Faktoren für eine Entscheidung im ersten Schritt (Kaufentscheidung) invariant gegenüber Änderungen in der Entscheidung im zweiten Schritt (Typ des Autos) sind, das heißt, falls die Parameterschätzer $\hat{\beta}_{j}, j=1, \ldots, p$, im Hazards-Modell unverändert gegenüber Änderungen in den bedingten Wahrscheinlichkeiten für eine bestimmte Ereignisart bleiben. Eine Änderung in den bedingten Wahrscheinlichkeiten für den Kauf bestimmter Automarken kann etwa durch den Konkurs oder durch besondere Werbemaßnahmen einer Firma eintreten.

Typ II-Situation: Diese Situation ist gegeben, falls unterschiedliche kausale Prozesse zum Eintreffen der verschiedenen Ereignisse führen. Die Hazardraten werden im Gegensatz zur Typ I-Situation getrennt für die unterschiedlichen Ereignisse modelliert. Es können zwar für die einzelnen Hazardraten die gleichen $p$ Kovariablen relevant sein, jedoch wird angenommen, dass die Parameterschätzer $\hat{\beta}_{l j}, l=1, \ldots, p, j=1, \ldots, m$, für die $m$ übergangsspezifischen Modelle voneinander unabhängig sind. Änderungen in den bedingten Wahrscheinlichkeiten für einen Ereignistyp lassen die Determinanten in den ereignisspezifischen Modellen, somit die Parameterschätzer $\hat{\beta}_{l j}, l=1, \ldots, p, j=1, \ldots, m$, unverändert. Werden etwa die Ursachen für unterschiedliche Ausfallsarten von Maschinen untersucht, so erscheint es plausibel, dass es unabhängige Ausfallsarten gibt, sodass die Beseitigung einer Ausfallsart keinerlei Konsequenzen auf die Ausfallsrate der anderen Ausfallsart hat. 
Diese Problemstellung entspricht dem häufig verwendeten competing risksModell, mit dem wir uns aufgrund seiner Bedeutung für Studienverlaufsanalysen in diesem Abschnitt näher auseinandersetzen wollen.

\section{Competing Risks}

Es sei $Y$ eine zufällige Zustandsvariable, die Werte aus der Menge der möglichen Endzustände $\{1, \ldots, m\}$ in einem competing risks-Modell annehmen kann, dann lässt sich die übergangsspezifische Hazardrate in den $j$-ten Zustand unter Berücksichtigung von Kovariablen folgendermaßen definieren:

$$
h_{j}(t \mid \mathbf{x})=\lim _{\Delta t \rightarrow 0} \frac{1}{\Delta t} P(t \leq T<t+\Delta t, Y=j \mid T \geq t, \mathbf{x})
$$

Die Gesamthazardrate zum Zeitpunkt $t$ ist

$$
h(t \mid \mathbf{x})=\sum_{j=1}^{m} h_{j}(t \mid \mathbf{x}) .
$$

Es ist zwar möglich, eine übergangsspezifische Überlebensfunktion mit

$$
S_{j}(t \mid \mathbf{x})=\exp \left(-\int_{0}^{t} h_{j}(u \mid \mathbf{x}) d u\right)
$$

zu formulieren, jedoch hat diese Funktion keine Interpretation einer Überlebensfunktion, da sie nicht die Wahrscheinlichkeit angibt, einen Zeitpunkt überlebt zu haben. Die Funktion $S_{j}$ wird daher in der Literatur meist als PseudoÜberlebensfunktion bezeichnet; sie wird einerseits für graphischen Modellüberprüfungen und andererseits zur Ermittlung der folgend definierten Größen verwendet.

Die Definition der (gemeinsamen) "übergangsspezifischen Dichte"

$$
\begin{aligned}
f_{j}(t \mid \mathbf{x}) & =\lim _{\Delta t \rightarrow 0} \frac{1}{\Delta t} P(t \leq T<t+\Delta t, Y=j \mid \mathbf{x}) \\
& =h_{j}(t \mid \mathbf{x}) S(t \mid \mathbf{x})=h_{j}(t \mid \mathbf{x}) \prod_{j=1}^{m} S_{j}(t \mid \mathbf{x})
\end{aligned}
$$

wird zur Berechnung der Wahrscheinlichkeit eines Übergangs in den $j$-ten Endzustand (bei gegebenem zeitunabhängigem Kovariablenvektor) benötigt:

$$
\pi_{j}(\mathbf{x})=\int_{0}^{\infty} f_{j}(t \mid \mathbf{x}) d t=P(Y=j \mid \mathbf{x})
$$


und

$$
\sum_{j=1}^{m} \pi_{j}(\mathbf{x})=1
$$

Mit 7.1 können etwa Studienabbruch-Wahrscheinlichkeiten eines Jahrgangs bzw. bestimmter Teilpopulationen geschätzt werden. Die bedingte erwartete Dauer (bei gegebenem zeitunabhängigem Kovariablenvektor) bis zum Übergang in den $j$-ten Endzustand wird mit

$$
E_{j}(T \mid \mathbf{x})=E(T \mid J=j ; \mathbf{x})=\int_{0}^{\infty} t \frac{1}{\pi_{j}(\mathbf{x})} f_{j}(t \mid \mathbf{x}) d t
$$

berechnet. Ausführlich werden die eben angeführten Beziehungen in Lancaster (1990) und Lawless (1982) diskutiert.

Zur Schätzung der "übergangsspezifischen Parameter" $\beta_{l j}, l=1, \ldots, p, j=$ $1, \ldots, m$, wird wieder die Maximierung der (partiellen) Likelihood Funktion verwendet. Unter der Annahme, dass die Ereigniszeiten der verschiedenen Ereignisarten (und die Zensierungszeiten) bedingt unabhängig sind, lässt sich die Likelihoodfunktion für das Mehr-Zustands-Modell anschreiben zu

$$
L=\prod_{i=1}^{n} \prod_{j=1}^{m}\left[h_{j}\left(t_{i} \mid \mathbf{x}_{i}\right)\right]^{\delta_{i j}} \exp \left(-\int_{0}^{t_{i}} h_{j}\left(u \mid \mathbf{x}_{i}\right) d u\right)
$$

wobei

$\delta_{i j}= \begin{cases}1 & \text { wenn das } i \text {-te Individuum zum Zeitpunkt } t_{i} \text { das Ereignis } j \text { hat } \\ 0 & \text { sonst. }\end{cases}$

Interpretiert man in Ein-Zustands-Modellen die Zensierung als zweite Ereignisart, so entspricht (5.6) genau (7.3) für zwei Zustände $(m=2)$. Ist die Typ II-Situation gegeben, das heißt, dass die übergangsspezifischen Hazardraten $h_{j}(t \mid \mathbf{x})$ von Parametervektoren $\boldsymbol{\beta}_{j}, j=1, \ldots, m$, abhängen, die keine Komponenten gemeinsam haben, kann jede Likelihoodfunktion

$$
L_{j}=\prod_{i=1}^{n}\left[h_{j}\left(t_{i} \mid \mathbf{x}_{i}\right)\right]^{\delta_{i j}} \exp \left(-\int_{0}^{t_{i}} h_{j}\left(u \mid \mathbf{x}_{i}\right) d u\right)
$$

getrennt maximiert und damit alle in den vorangegangenen Abschnitten behandelten Methoden für Modelle mit einem Endzustand verwendet werden. In einem Cox-Modell ergeben sich unter Berücksichtigung von (zeitabhängigen) Kovariablen etwa die übergangsspezifischen Hazardraten

$$
h_{j}[t \mid \mathbf{x}(t)]=h_{0 j}(t) \exp \left(\mathbf{x}^{\prime}(t) \boldsymbol{\beta}_{j}\right) \quad j=1, \ldots, m .
$$


Die Likelihood-Funktion (7.3) muss simultan maximiert werden, falls die Annahme von Parameterrestriktionen wie etwa die Annahme von proportionalen Basis-Hazardraten

$$
h_{0 j}(t)=h_{0}(t) \exp \left(\boldsymbol{\beta}_{0 j}\right) \quad j=1, \ldots, m
$$

oder von gleichen Regressionskoeffizienten $\beta_{l 1}=\ldots=\beta_{l m}$ gerechtfertigt ist. Die simultane Maximierung ist etwa in TDA möglich.

Die Maximierung von (7.4) entspricht genau der Maximierung von (5.6), wobei alle vom zu analysierenden Ereignis verschiedenen Ereignisse als (nichtinformativ bzw. bedingt unabhängig) zensierte Fälle interpretiert werden können. Ist die Annahme von bedingt unabhängigen Ereignissen erfüllt, spricht man von (bedingt) unabhängigen competing risks. Es wird in diesem Modell somit angenommen, dass Individuen, die ein besonders hohes (oder niedriges) Risiko für das zu analysierende Ereignis haben, weder mehr noch weniger gefährdet für irgendein anderes Ereignis als andere Individuen sind - bedingt unter den einbezogenen Kovariablen.

Hachen (1988) und Prentice und Kalbfleisch (1979) erklären, dass bei Verletzung dieser Annahme die Parameterschätzer nur unter den gegebenen Studienbedingungen valide sind und auch entsprechend interpretiert werden müssen. Ist die Annahme von bedingt unabhängigen Ereignissen verletzt, dann führen Veränderungen in der bedingten Wahrscheinlichkeit eines Ereignisses zu veränderten Parameterschätzern für die anderen Ereignisarten.

Im Fall von Studienverlaufsanalysen mit den Endzuständen "abgebrochen" und "erfolgreich abgeschlossen" eignet sich die Modellierung eines competing risks-Modells; siehe Booth und Satchell (1995). Die Parameterschätzer sind jedoch mit etwas Vorsicht zu interpretieren, da - auch nach Einbeziehung von Kovariablen, von denen vermutet werden kann, dass sie beide Ereignisse beeinflussen (wie etwa die Erwerbstätigkeit und die schulische Vorbildung) nicht unbedingt von (bedingt) unabhängigen Ereignissen "Studienabbruch" und "Studienabschluss" ausgegangen werden kann. Es liegt etwa die Vermutung nahe, dass Studienabbrecher eher länger für das Studium gebraucht hätten und diese Abhängigkeit nicht restlos von den einbezogenen Kovariablen erklärt wird. Eine Verletzung dieser Annahme bei Analysen der Studiendauer bis zum erfolgreichen Studienabschluss bedeutet, dass eine Änderung der Studienabbruch-Wahrscheinlichkeit eine Änderung der Parameterschätzer im Modell für den Studienabschluss zur Folge hätte.

Modelle, die die Abhängigkeit von Ereignisarten berücksichtigen, haben in praktischen Anwendungen bisher kaum Verwendung gefunden; siehe Allison (1998). 
Um einen Eindruck davon zu bekommen, wie sensitiv die Parameterschätzer auf Änderungen der Hazardraten der anderen Ereignisse reagieren, schlägt Allison (1998) Sensitivitätsanalyse vor, in der das Modell unter zwei Extremannahmen nochmals geschätzt wird. Der erste Extremfall geht von der Annahme aus, dass die (durch ein anderes als das zu analysierende Ereignis) zensierten Fälle solche Individuen sind, die sonst eine sehr lange Verweildauer hätten. Ihre Zensierungszeit wird in diesem Fall mit der maximalen beobachteten Zensierungszeit gleich gesetzt. Der zweite Fall geht von der Annahme aus, dass die zensierten Fälle ansonsten unmittelbar nach der Zensierung das betrachtete Ereignis gehabt hätten. Ihre eigentliche Zensierungszeit wird in diesem Fall in eine Ereigniszeit umgewandelt. Erhält man bei der Schätzung der beiden Extremfälle ähnliche Parameterschätzer (und damit Interpretation der einbezogenen Kovariablen) wie im Ausgangsmodell, so kann der Annahme von bedingt unabhängigen Ereignissen vertraut werden. Je nach Analyse scheint es manchmal nur sinnvoll, einen der beiden Extremfälle zu überprüfen. Bei Studienverlaufsanalysen macht etwa nur die Implementierung des ersten Falls Sinn, da Studienabbrecher mit Sicherheit nicht unmittelbar vor Studienabschluss ihr Studium abbrechen.

Um zu überprüfen, ob sich die ereignisspezifischen Parameter tatsächlich für die verschiedenen Ereignisarten unterscheiden, kann die Hypothese $\boldsymbol{\beta}_{j}=\boldsymbol{\beta}$ für alle $j=1, \ldots, m$ mit Hilfe eines Likelihood-Quotienten-Tests überprüft werden. Zur Ermittlung der Likelihood-Quotienten-Teststatistik wird ein EinZustands-Modell geschätzt, das alle Ereignisarten gleich behandelt, und dann der Wert der logarithmierten Likelihoodfunktion $l(\hat{\boldsymbol{\beta}})$ ermittelt. Danach wird die Summe der logarithmierten Werte $l\left(\hat{\boldsymbol{\beta}}_{j}\right)$ der getrennt maximierten Likelihoodfunktionen (7.4) berechnet und die Teststatistik

$$
G=2\left[l(\hat{\boldsymbol{\beta}})-\sum_{j=1}^{m} l\left(\hat{\boldsymbol{\beta}}_{j}\right)\right]
$$

ermittelt. Diese Teststatistik ist dann $\chi^{2}(m p-p)$ verteilt.

Zur Überprüfung der Annahme, dass die Regressionskoeffizienten einer Kovariablen in zwei ereignisspezifischen Modellen gleich sind, $H_{0}: \beta_{11}=\beta_{12}$, kann die Wald-Teststatistik

$$
\frac{\left(\hat{\beta}_{11}-\hat{\beta}_{12}\right)^{2}}{\hat{V} a r\left(\hat{\beta}_{11}\right)+\hat{V} a r\left(\hat{\beta}_{12}\right)}
$$

verwendet werden, die $\chi^{2}(1)$ verteilt ist.

Competing risks-Modelle behandeln unter anderem Allison (1984), Blossfeld et al. (1986), Hachen (1988), Prentice und Kalbfleisch (1979), Prentice et al. (1978) und Vermunt (1997) ausführlich. 


\subsection{Erweiterung der Mehr-Zustands-Modelle auf den Mehr-Episoden-Fall}

In diesem Abschnitt werden die Methoden für die Mehr-Zustands-Modelle auf den Fall von mehreren Episoden erweitert. Mit $\left(T_{k}, J_{k}\right), k=1,2, \ldots$, werden Ereigniszeit und Art des Ereignisses, das die $k$-te Episode beendet, bezeichnet. Da die zufälligen Ereigniszeiten vom Beginn der Beobachtungsperiode gemessen werden, gilt $0=T_{0}<T_{1}<T_{2}<\ldots$.

Es sei $Y_{k}$ eine zufällige Zustandsvariable, die Werte aus der Menge der möglichen Endzustände $\{1, \ldots, m\}$ der $k$-ten Episode in einem Mehr-ZustandsModell annehmen kann, dann wird die übergangsspezifische Hazardrate der $k$-ten Episode in den $j$-ten Zustand in Abhängigkeit von der Dauer $t$ seit Prozessbeginn folgendermaßen definiert:

$$
h_{j}^{k}\left(t \mid \mathbf{x}_{k}, H_{k-1}\right)=\lim _{\Delta t \rightarrow 0} \frac{1}{\Delta t} P\left(t \leq T_{k}<t+\Delta t, Y_{k}=j \mid T_{k} \geq t, H_{k-1}, \mathbf{x}_{k}\right),
$$

wobei $\mathbf{x}_{k}$ den Kovariablenvektor der $k$-ten Episode bezeichnet und in $H_{k-1}$ die Vorgeschichte des Prozessverlaufs bis zum Zeitpunkt $t_{k-1}$ zusammengefasst wird, also

$$
H_{k-1}=\left\{y_{0}, t_{1}, y_{1}, \mathbf{x}_{1}, \ldots, t_{k-1}, y_{k-1}, \mathbf{x}_{k-1}\right\} \text {. }
$$

Es sei hier angemerkt, dass die Hazardrate für den Mehr-Zustands- und MehrEpisoden-Fall sowie die zu den Kovariablen gehörigen Parameter in der Literatur gelegentlich explizit in Abhängigkeit vom Ausgangszustand der $k$-ten Episode $y_{k-1}$ formuliert werden, während in der Darstellung (7.5) der Zustand $y_{k-1}$ in der Vorgeschichte $H_{k-1}$ enthalten ist; siehe etwa Vermunt (1997).

Für nicht existierende $(k, j)$-Kombinationen und für $t<t_{k-1}$ ist $h_{j}^{k}()=$.0 . Da für die Schätzung der Parameter der übergangsspezifischen Hazardrate $h_{j}^{k}($.$) nur jene Individuen herangezogen werden, die sich in der k$-ten Episode befinden (und somit schon $k-1$ Ereignisse erlebt haben), spricht man im Falle von (7.5) auch von einer bedingten Hazardrate.

Sei $U_{k}=T_{k}-T_{k-1}$ die Dauer in der $k$-ten Episode bis zum Eintreffen des $k$-ten Ereignisses, dann ist

$$
h_{j}^{k}\left(u \mid \mathbf{x}_{k}, H_{k-1}\right)=\lim _{\Delta t \rightarrow 0} \frac{1}{\Delta t} P\left(u \leq U_{k}<u+\Delta u, Y_{k}=j \mid U_{k} \geq u, H_{k-1}, \mathbf{x}_{k}\right)
$$

die übergangsspezifische Hazardrate der $k$-ten Episode in Abhängigkeit von der Episodendauer. 
Die Gesamthazardrate $h^{k}\left(t \mid \mathbf{x}_{k}, H_{k-1}\right)$, in der k-ten Episode den Zustand $y_{k-1}$ $\mathrm{zu}$ verlassen, erhält man mit

$$
h^{k}\left(t \mid \mathbf{x}_{k}, H_{k-1}\right)=\sum_{j=1}^{m} h_{j}^{k}\left(t \mid \mathbf{x}_{k}, H_{k-1}\right) .
$$

Die Überlebensfunktion im Mehr-Episoden- und Mehr-Zustands-Fall gibt die Wahrscheinlichkeit an, ausgehend vom Zustand $y_{k-1}$ den Zeitpunkt $t$ zu überleben, das heißt, dass bis zu diesem Zeitpunkt der $k$-te Übergang noch nicht stattgefunden hat. Es gilt für $t \geq t_{k-1}$

$$
\begin{aligned}
S^{k}\left(t \mid \mathbf{x}_{k}, H_{k-1}\right) & =P\left(T_{k}>t \mid \mathbf{x}_{k}, H_{k-1}\right) \\
& =\exp \left[-\int_{t_{k-1}}^{t} h^{k}\left(u \mid \mathbf{x}_{k}, H_{k-1}\right) d u\right]
\end{aligned}
$$

und mit (7.6) ist

$$
\begin{aligned}
S^{k}\left(t \mid \mathbf{x}_{k}, H_{k-1}\right) & =\exp \left[-\int_{t_{k-1}}^{t} \sum_{j=1}^{m} h_{j}^{k}\left(u \mid \mathbf{x}_{k}, H_{k-1}\right) d u\right] \\
& =\prod_{j=1}^{m} \exp \left[-\int_{t_{k-1}}^{t} h_{j}^{k}\left(u \mid \mathbf{x}_{k}, H_{k-1}\right) d u\right] .
\end{aligned}
$$

Die "übergangsspezifische Dichtefunktion" der $k$-ten Episode erhält man zu

$$
\begin{aligned}
f_{j}^{k}\left(t \mid \mathbf{x}_{k}, H_{k-1}\right) & =\lim _{\Delta t \rightarrow 0} \frac{1}{\Delta t} P\left(t \leq T_{k}<t+\Delta t, Y_{k}=j \mid \mathbf{x}_{k}, H_{k-1}\right) \\
& =h_{j}^{k}\left(t \mid \mathbf{x}_{k}, H_{k-1}\right) S^{k}\left(t \mid \mathbf{x}_{\mathbf{k}}, H_{k-1}\right) .
\end{aligned}
$$

Die Parameterschätzer werden unter der Annahme von bedingt unabhängigen Ereignisarten (und Zensierungszeiten) durch Maximierung der Likelihood-Funktion für den Mehr-Zustands- und Mehr-Episoden-Fall erhalten:

$$
L=\prod_{k} \prod_{j=1}^{m} \prod_{i=1}^{n}\left[h_{j}^{k}\left(t_{i k} \mid H_{i, k-1}, \mathbf{x}_{i k}\right)\right]^{\delta_{i k j}}\left[S_{j}^{k}\left(t_{i k} \mid H_{i, k-1}, \mathbf{x}_{i k}\right)\right]^{\epsilon_{i k}}
$$

mit

$$
\begin{gathered}
\delta_{i k j}= \begin{cases}1 & \text { falls die } k-t e \text { Episode von Individuum } i \\
0 & \begin{array}{l}
\text { zum Zeitpunkt } t_{i k} \text { im Zustand } j \text { endet }
\end{array}\end{cases} \\
\epsilon_{i k}= \begin{cases}1 & \text { falls Individuum } i \text { die } k \text {-te Episode überlebt } \\
0 & \text { sonst }\end{cases}
\end{gathered}
$$


Details zur Herleitung der Likelihood-Funktion sind beispielsweise in Blossfeld et al. (1986) oder Hamerle (1989) zu finden.

Die Überlebensfunktion, die übergangsspezifische Dichtefunktion der $k$-ten Episode und die Likelihoodfunktion können auch in Abhängigkeit der Episodendauer $u=t_{k}-t_{k-1}$ angeschrieben werden.

Die Likelihood-Funktion (7.7) kann getrennt für jeden $(k, j)$-Übergang maximiert werden, wenn die Hazardraten $h_{j}^{k}($.$) von Parametervektoren \beta_{j}^{k}$ abhängen, die keine Komponenten gemeinsam haben. Zur Analyse der $k$-ten Episode werden nur jene Individuen herangezogen, die die Ereignisse $1,2, \ldots, k-1$ bereits erlebt haben. Diese Individuen gehen im Fall der Modellierung in Abhängigkeit von der Dauer $t$ seit Prozessbeginn als links abgeschnittene Beobachtungen in die Schätzung ein, denn es ist für jedes Individuum in der $k$-ten Episode bekannt, dass es den Zeitpunkt $t_{k-1}$, den Beginn der $k$-ten Episode, überlebt hat; siehe Abschnitt 3.2. In der $k$-Episode selbst werden die vom interessierenden Endzustand verschiedenen Ereignisarten analog zu Abschnitt 7.1 als rechts-zensierte Daten behandelt.

Zur Modellierung der Hazardraten $h_{j}^{k}($.$) kommen dann die in den vergangenen$ Abschnitten diskutierten Ansätze in Frage, für das Cox-Modell (in Abhängigkeit der Dauer $t$ seit Prozessbeginn) beispielsweise

$$
h_{j}^{k}\left(t \mid H_{k-1}, \mathbf{x}_{k}\right)=h_{0 j}^{k}(t) \exp \left(\mathbf{x}_{k}^{\prime} \beta_{j}^{k}\right),
$$

wobei der relevante Teil der Vorgeschichte $H_{k-1}$ in den aktuellen Kovariablenvektor $\mathbf{x}_{k}$ aufgenommen wird.

Bei der getrennten Maximierung der Hazardraten $h_{j}^{k}($.$) wird davon ausge-$ gangen, dass die Parametervektoren $\boldsymbol{\beta}_{j}^{k}$ episoden- und übergangsspezifisch sind und mit anderen $(k, j)$-Übergängen keine Komponenten gemeinsam haben. Besteht etwa die Annahme, dass manche Komponenten der Vektoren $\boldsymbol{\beta}_{j}^{k}$ für verschiedene Episoden und Übergänge gleich sind, muss die Likelihoodfunktion simultan maximiert werden, etwa mit TDA. Im Fall von wiederholten Ereignissen (zum Beispiel Berufsepisoden) ist es manchmal sinnvoll, das Mehr-Episoden-Modell so weit einzuschränken, dass für alle $k$ Episoden $\boldsymbol{\beta}^{1}=\boldsymbol{\beta}^{2}=\ldots=\boldsymbol{\beta}^{k}$ und $h_{0}^{1}(t)=h_{0}^{2}(t)=\ldots=h_{0}^{k}(t)$ gilt. Diese Einschränkungen entsprechen dem Fall, dass die Episoden jedes Individuums als eigene unabhängige Beobachtungen in das Modell eingehen, wobei die Beobachtungen in späteren Episoden als links abgeschnittene Fälle in das Modell einbezogen werden. Werden die Hazardraten in den jeweiligen Episoden nicht in Abhängigkeit von der Zeit $t$ seit Prozessbeginn modelliert, sondern in Abhängigkeit von der Episodendauer $t-t_{k-1}$, so entspricht dies exakt der Modellierung im Ein-Episodenmodell, wobei jede Episode eines Individuums als 
eigene unabhängige Beobachtung in das Modell eingeht. Die Annahme, dass die Episoden eines Individuums wie unabhängige Beobachtungen behandelt werden können, ist jedoch in vielen Fällen nicht gerechtfertigt. In diesem Fall führt dieses Modell zu verzerrten Schätzern und verzerrten Standardfehlern. Blossfeld et al. (1986) verwenden beispielsweise diese Modellierung zur Analyse von Berufsepisoden.

Häufig implementierte Spezialfälle der allgemeinen Hazardfunktion (7.5) sind progressive Mehr-Zustands und Mehr-Episoden-Modelle sowie (heterogene) Markov- und Semi-Markov-Modelle. Im progressiven Modell haben die Episoden eine eindeutige Reihenfolge und ein Individuum kann jeden weiteren $\mathrm{Zu}$ stand nur dann erreichen, wenn es sich gerade im der Reihenfolge entsprechenden Zustand "davor" befindet. Somit beinhaltet die Information über den derzeitigen Zustand automatisch die Information, welche und wieviele Zustände in welcher Reihenfolge vor dem jetzigen Zustand durchlaufen worden sind. Die Modellierung vereinfacht sich, da in $H_{k-1}$ diese Information nicht mehr inkludiert werden muss. Der Ausgangszustand der $k$-ten Episode ist etwa automatisch bekannt. Die Phasen eines Studiums, wo beispielsweise der zweite Abschnitt nur nach Absolvierung des ersten Abschnitts abgeschlossen werden kann, oder die Karriereleiter in einer Firma sind Beispiele für progressive Mehr-Episoden-Modelle.

Im Markov-Modell wird angenommen, dass die Hazardrate für einen Übergang vom Zustand $l$ in den Zustand $j$ nicht von der Vergangenheit $H_{k-1}$ und nicht von der Nummer der Episode abhängt. Beim stationären Modell wird zusätzlich angenommen, dass die Hazardrate nicht von der Dauer $t$ seit Prozessbeginn abhängt.

Semi-Markov-Modelle erlauben in Erweiterung des Markov-Modells noch, dass die Hazardrate für einen Übergang vom Zustand $l$ in den Zustand $j$ auch vom Zeitpunkt $t_{l}$ des letzten Übergangs abhängen kann. Im Gegensatz zum Markov-Modell kann das Semi-Markov-Modell auch in Abhängigkeit von der Verweildauer $U^{k}=U^{k}-U^{k-1}$ in der Episode angeschrieben werden. Bei Studienverlaufsanalysen bedeutet das, dass in Semi-Markov-Modellen die gesamte Studiendauer auch von der Dauer des ersten Abschnitts (Zeitpunkt des Übergangs vom ersten in den zweiten Studienabschnitt) abhängen kann.

Wei et al. (1989) schlagen als Alternative zu Modell (7.5) vor, die Hazardrate für das Eintreffen des $k$-ten Ereignisses wie Ein-Episoden-Modelle zu behandeln, also die marginale Hazardrate

$$
h_{j}^{k}(t \mid \mathbf{x})=\lim _{\Delta t \rightarrow 0} \frac{1}{\Delta t} P\left(t \leq T_{k}<t+\Delta t, Y_{k}=j \mid T_{k} \geq t, \mathbf{x}\right)
$$

zu modellieren. Bei diesem Modell wird auf die Information verzichtet, dass 
Individuen, die zum Zeitpunkt $t$ noch nicht in der $k$-ten Episode sind, nicht zur Risikomenge $R(t)$ für das $k$-te Ereignis gehören. In ihrer Arbeit präsentieren die Autoren einen robusten Schätzer für die Varianz-Kovarianzmatrix der erhaltenen Parameterschätzer $\hat{\boldsymbol{\beta}}^{k}$, der Abhängigkeiten zwischen den Ereigniszeiten jedes Individuums erlaubt. Mit Hilfe dieses Schätzers können dann etwa Tests über die Gleichheit von Regressionskoeffizienten $\beta_{l j}^{1}=\ldots=\beta_{l j}^{k}, l=$ $1, \ldots, p, j=1 \ldots, m$, durchgeführt werden und bei Annahme dieser Hypothese der für alle Episoden gleiche Parameter $\beta_{l j}$ geschätzt werden; siehe Wei et al. (1989), Allison (1998), Therneau (1997) und Oakes (1997). Die Anwendung dieser Methoden wird beispielsweise in SAS ermöglicht.

Mit (bedingten) Mehr-Zustands- und Mehr-Episoden-Modellen setzen sich unter anderem Kalbfleisch und Prentice (1980), Prentice et al. (1981), Blossfeld et al. (1986), Hamerle (1989), Hougaard (1999b) sowie Vermunt (1997) auseinander. 


\section{Kapitel 8}

\section{Diskrete Ereignisdatenmodelle}

Im Abschnitt 6.2 wurde auch auf die partielle Likelihoodschätzung für den Fall eingegangen, dass mehrerer Ereignisse auf einen Zeitpunkt fallen - man spricht in diesem Fall von Bindungen. Ist die Anzahl von Ereignissen an gleichen Zeitpunkten sehr groß, weil die Messungen unpräzise sind oder die Ereignisse tatsächlich nur an diskreten Zeitpunkten eintreffen können, wird der Rechenaufwand für die Schätzung dieser Modelle sehr hoch. In diesen Fällen ist es geeigneter, diskrete Ereignisdatenmodelle zu implementieren. Ist etwa im Fall von Studienverlaufsanalysen nur das Semester des Abschlusses bzw. des Abbruchs bekannt, so empfiehlt sich die Implementierung von diskreten Ereignisdatenmodellen.

Bei der diskreten Modellierung von Ereignisdaten wird angenommen, dass $T$ eine diskrete Zufallsvariable ist, die die Dauer bis zum Eintreffen eines Ereignisses repräsentiert. Die Zeitachse wird in diskrete Zeitpunkte $t_{1}<t_{2}<\ldots$ unterteilt. Im Falle von "echten" diskreten Ereignis- bzw. Zensierungszeitpunkten bedeutet $T=t_{l}$, dass das Ereignis zum Zeitpunkt $t_{l}$ eingetroffen ist; bei unpräzisen Messungen bedeutet $T=t_{l}$, dass das Ereignis im Intervall $\left[t_{l-1}, t_{l}\right)$ eingetroffen ist. Dann ist die Wahrscheinlichkeit, dass ein Ereignis zum Zeitpunkt $t_{l}$ (bzw. im Intervall $l$-ten Intervall $\left[t_{l-1}, t_{l}\right)$ ) eintrifft, gegeben mit

$$
f\left(t_{l}\right)=P\left(T=t_{l}\right) .
$$

Die Überlebensfunktion $S\left(t_{l}\right)$, die die Wahrscheinlichkeit angibt, dass das Ereignis nach dem Zeitpunkt $t_{l}$ bzw. nach dem $l$-ten Intervall eintrifft, ist

$$
S\left(t_{l}\right)=P\left(T>t_{l}\right)=\sum_{j>l} f\left(t_{j}\right) .
$$

Es sei hier angemerkt, dass manche Autoren wie etwa Hamerle und Tutz (1989) die Überlebensfunktion mit $S(t)=P(T \geq t)$ definieren. 
Die Hazardrate zum Zeitpunkt $t_{l}$ ist gegeben mit

$$
\lambda\left(t_{l}\right)=P\left(T=t_{l} \mid T \geq t_{l}\right)=\frac{f\left(t_{l}\right)}{S\left(t_{l-1}\right)} .
$$

Im Falle von diskreten Modellen ist die Hazardrate somit die bedingte Wahrscheinlichkeit, dass ein Individuum zum Zeitpunkt $t_{l}$ bzw. im $l$-ten Zeitintervall ein Ereignis erlebt, gegeben, dass für das Individuum nicht vor dem Zeitpunkt $t_{l}$ bzw. vor dem $l$-ten Intervall das Ereignis schon eingetroffen ist. Mit

$$
\lambda\left(t_{l}\right)=\frac{S\left(t_{l-1}\right)-S\left(t_{l}\right)}{S\left(t_{l-1}\right)}=1-\frac{S\left(t_{l}\right)}{S\left(t_{l-1}\right)}
$$

erhalten wir die folgenden Beziehungen zwischen Überlebensfunktion, Hazardrate und Ereigniswahrscheinlichkeit:

$$
\begin{aligned}
& S\left(t_{l}\right)=\prod_{k=1}^{l}\left[1-\lambda\left(t_{k}\right)\right] \\
& f\left(t_{l}\right)=\lambda\left(t_{l}\right) S\left(t_{l-1}\right)=\lambda\left(t_{l}\right) \prod_{k=1}^{l-1}\left[1-\lambda\left(t_{k}\right)\right]
\end{aligned}
$$

Um den Einfluss von erhobenen Kovariablen zu ermitteln, wird wieder die Hazardrate in Abhängigkeit von den Kovariablen modelliert.

\subsection{Logistisches- und gruppiertes Cox-Modell}

Im Falle von "echten" diskreten Ereigniszeitpunkten (Gehaltsauszahlungen in einem Unternehmen erfolgen jeden Monat zum gleichen Zeitpunkt) wird häufig das logistische Modell, das von Cox (1972) vorgeschlagen worden ist, gewählt:

$$
\ln \left(\frac{\lambda\left[t_{l} \mid \mathbf{x}\left(t_{l}\right)\right]}{1-\lambda\left[t_{l} \mid \mathbf{x}\left(t_{l}\right)\right]}\right)=\alpha_{l}+\mathbf{x}\left(t_{l}\right)^{\prime} \boldsymbol{\beta}
$$

Das Modell (8.1) ist hier in verallgemeinerter Form angeschrieben, die auch die Einbeziehung von zeitveränderlichen Variablen erlaubt. Im Falle von zeitkonstanten Kovariablen gilt, dass die Werte der Kovariablen zu allen diskreten Zeitpunkten $t_{l}$ gleich sind. Zeitabhängige Variablen können analog zu Abschnitt 6.3 unterschiedlich definiert und in das Modell integriert werden; siehe auch Hamerle und Tutz (1989).

Die Hazardrate für dieses Modell, die bei diskreten Modellen eine bedingte Wahrscheinlichkeit ist, erhält man zu

$$
\lambda\left[t_{l} \mid \mathbf{x}\left(t_{l}\right)\right]=P\left(T=t_{l} \mid T \geq t_{l}, x\left(t_{l}\right)\right)=\frac{\exp \left(\alpha_{l}+\mathbf{x}\left(t_{l}\right)^{\prime} \boldsymbol{\beta}\right)}{1+\exp \left(\alpha_{l}+\mathbf{x}\left(t_{l}\right)^{\prime} \boldsymbol{\beta}\right)} .
$$


Das logistische Modell spezifiziert $\ln [(P / 1-P)]$ als lineare Funktion der Kovariablen. Das Verhältnis $P /(1-P)$ wird im Englischen odds genannt; der gleiche Begriff wird in Ermangelung eines deutschen Wortes auch meist in der deutschsprachigen Literatur gebraucht. Die odds sind eine andere Skalierung für die Wahrscheinlichkeit $P$. Während für diese $0 \leq P \leq 1$ gilt, sind die odds Zahlen aus $[0, \infty)$.

Die Kovariablen im Modell (8.1) haben die Interpretation, dass unter Kontrollierung aller anderen Kovariablen eine Erhöhung von $x_{j}, j=1, \ldots, p$, um eine Einheit die $\log$-odds für das Eintreffen eines Ereignisses um $\beta_{j}$ bzw. die odds um $100\left(\exp \left(\beta_{j}\right)-1\right) \%$ verändert.

Sind alle Kovariablen konstant über die Zeit, dann bezeichnet man das Modell (8.1) als proportionales odds-Modell, da die odds von einem Individuum ein Vielfaches der odds eines anderen Individuums sind. In dieser Hinsicht ist das logistische Modell dem in den Kapiteln 5 und 6 behandelten proportionalen Hazards-Modellen, in denen die Hazardraten von Individuen mit unterschiedlichen Ausprägungen der Kovariablen proportional zueinander sind und das Verhältnis der Hazardraten somit unabhängig von der Zeit ist, ähnlich. Das Einbeziehen von zeitveränderlichen Variablen führt zu einem nicht proportionalen odds-Modell.

Die Parameter $\alpha_{l}$ repräsentieren ähnlich wie im semiparametrischen Cox-Modell bei stetig gemessener Zeit eine Grund-Hazardrate ohne Berücksichtigung der Kovariablen, die allen Individuen gemeinsam ist. Im Unterschied zum CoxModell wird das Modell (8.1) mit der ML-Methode geschätzt und werden daher explizite Schätzer für $\alpha_{l}$ ermöglicht. Manchmal ist es sinnvoll, die Parameter $\alpha_{l}$, die die Effekte der Zeit auf die odds für ein Ereignis modellieren, näher zu spezifizieren. Die Parameter $\alpha_{l}$ werden häufig ersetzt durch: (a) $\alpha t_{l}$ (lineare Effekte der Zeit auf die $\log$-odds), (b) $\alpha \ln t_{l}$ oder (c) $\alpha t_{l}^{2}$.

Wie erwähnt wird im diskreten logistischen Modell davon ausgegangen, dass die Ereignisse nur an diskreten Zeitpunkten eintreffen können. In vielen Anwendungen hingegen liegt eine stetige Verweildauer zugrunde, aufgrund von unpräzisen Messungen fällt jedoch eine große Anzahl von Ereignissen auf die gleichen Zeitpunkte. Wird etwa die Studiendauer in Semestern gemessen, fallen viele Abschlüsse bzw. Abbrüche in ein Semester.

In diesem Fall wird häufig das Cox-Modell für stetige Verweildauern zugrunde gelegt und die Daten in Intervallen gruppiert. Daraus resultiert das (unter Berücksichtigung der Einbeziehung von zeitabhängigen Variablen verallgemeinert formulierte) gruppierte Cox-Modell:

$$
\ln \left\{-\ln \left(1-\lambda\left[t_{l} \mid \mathbf{x}\left(t_{l}\right)\right]\right\}=\alpha_{l}+\mathbf{x}\left(t_{l}\right)^{\prime} \boldsymbol{\beta},\right.
$$


wobei die Parameter $\alpha_{l}$ wieder die Grund-Hazardrate repräsentieren. Wird beispielsweise $\alpha_{l}=\alpha t_{l}$ gesetzt, so wird ein Gompertz-Modell geschätzt und bei $\alpha_{l}=\alpha \ln t_{l}$ ein Weibull-Modell mit gruppierten Daten. Die Parameter $\boldsymbol{\beta}$ bleiben bei der Diskretisierung unverändert gegenüber dem zugrundeliegenden proportionalen Hazards-Modell. Das bedeutet nicht, dass man für die beiden Modelle die gleichen Werte für die Parameterschätzer erhält, sondern dass beide Modelle die gleichen zugrundeliegenden Parameter schätzen und die $\mathrm{Pa}$ rameterschätzer direkt vergleichbar sind und die gleiche Interpretation haben.

Für die diskrete Hazardrate erhält man

$$
\lambda\left[t_{l} \mid \mathbf{x}\left(t_{l}\right)\right]=1-\exp \left[-\exp \left(\alpha_{l}+\mathbf{x}\left(t_{l}\right)^{\prime} \boldsymbol{\beta}\right)\right] .
$$

Während für das zugrundeliegende Cox-Modell (mit zeitkonstanten Kovariablen) die Eigenschaft der proportionalen Hazards vorliegt, gilt diese Eigenschaft für das gruppierte Cox-Modell nicht mehr. Die Proportionalität der logarithmierten Überlebensfunktionen überträgt sich jedoch auf das gruppierte Cox-Modell:

$$
\frac{\ln S\left(t_{l} \mid \mathbf{x}_{i}\right)}{\ln S\left(t_{l} \mid \mathbf{x}_{j}\right)}=\exp \left[\left(\mathbf{x}_{i}-\mathbf{x}_{j}\right]^{\prime} \boldsymbol{\beta}\right]
$$

\subsection{Maximum-Likelihood-Schätzung}

Diskrete Modelle werden im Allgemeinen mit der ML-Methode geschätzt. Unter der Annahme, dass der Zensierungsmechanismus bedingt unabhängig ist, trägt analog zu den stetigen Modellen ein Individuum, das ein Ereignis erlebt hat, mit $f\left(t_{l} \mid \mathbf{x}\right)$ und ein rechts-zensiertes Individuum mit $S\left(t_{l} \mid \mathbf{x}\right)$ zur Likelihood-Funktion bei. Mit

$$
f\left(t_{l} \mid \mathbf{x}\right)=\lambda\left(t_{l} \mid \mathbf{x}\right) \prod_{k=1}^{l-1}\left[1-\lambda\left(t_{k} \mid \mathbf{x}\right)\right]=\frac{\lambda\left(t_{l} \mid \mathbf{x}\right)}{1-\lambda\left(t_{l} \mid \mathbf{x}\right)} \prod_{k=1}^{l}\left[1-\lambda\left(t_{k} \mid \mathbf{x}\right)\right]
$$

und $t_{l_{i}}$ als jenen Zeitpunkt (bzw. $\left[t_{l_{i}-1}, t_{l_{i}}\right.$ ) als jenes Zeitintervall), an dem für das $i$-te Individuum das Ereignis eingetroffen ist, erhalten wir für die Likelihoodfunktion

$$
L=\prod_{i=1}^{N}\left[\left(\frac{\lambda\left(t_{l_{i}} \mid \mathbf{x}_{i}\right)}{1-\lambda\left(t_{l_{i}} \mid \mathbf{x}_{i}\right)}\right)^{\delta_{i}} \prod_{k=1}^{l_{i}}\left[1-\lambda\left(t_{k} \mid \mathbf{x}_{i}\right)\right]\right]
$$

wobei $\delta_{i}$ der Zensierungsindikator ist. Wird mit $\mathbf{y}_{i}$ ein Vektor bezeichnet, der $l_{i}$ Indikator-Variablen enthält, die den Wert 1 annehmen, falls das $i$-te Individuum in $T=t_{l}$ eine Ereignis erlebt, und sonst 0 sind - somit sind die ersten 
$l_{i}-1$ Elemente von $\mathbf{y}_{i}$ gleich Null und das letzte Element gleich dem Wert des Zensierungsindikators $\delta_{i}$ - dann lässt sich die Likelihood-Funktion anschreiben zu:

$$
L=\prod_{i=1}^{N} \prod_{k=1}^{l_{i}}\left[\left(\frac{\lambda\left(t_{l_{i}} \mid \mathbf{x}_{i}\right)}{1-\lambda\left(t_{l_{i}} \mid \mathbf{x}_{i}\right)}\right)^{y_{i k}}\left[1-\lambda\left(t_{k} \mid \mathbf{x}_{i}\right)\right]\right]
$$

Diese Likelihood-Funktion entspricht der Likelihood-Funktion für Regressionsmodelle mit binären Responsevariablen - mit dem Unterschied, dass ein Individuum in diese Likelihood-Funktion nicht mit einer Beobachtung sondern mit $l_{i}$ Beobachtungen eingeht. Somit können diskrete Ereignisdatenmodelle mit Hilfe der gängigen Software für logistische Regressionsmodelle geschätzt werden. Für diesen Zweck wird der Datensatz des $i$-ten Individuums analog zum stückweise konstanten Hazards-Modell aus Abschnitt (5.4) in $l_{i}$ Datensätze unterteilt, die als eigenständige Beobachtungen in die Schätzung eingehen. Zeitveränderliche Variablen können wieder insofern leicht implementiert werden, indem sie zu jedem diskreten Zeitpunkt $t_{l}$ (bzw. zu Beginn jedes Zeitintervalls $\left[t_{l-1}, t_{l}\right)$ ) aktualisiert werden.

Ausführlich diskutieren Hamerle und Tutz (1989) diskrete Modelle zur Analyse von Verweildauern; unter anderem wird auch die Erweiterung auf den Mehr-Zustands- und Mehr-Episoden-Fall behandelt. Diese Erweiterung lässt sich analog zu Kapitel 7 auch für diskrete Modelle formulieren. Weiters setzen sich beispielsweise Allison (1982), Allison (1998), Vermunt (1997) und Yamaguchi (1991) mit diskreten Modellen auseinander. 
Günther Sedlacek - 978-3-631-75405-4

Downloaded from PubFactory at 01/11/2019 05:23:57AM

via free access 


\section{Teil B \\ Empirische Analyse der Studiendauer und des Studienabbruch-Risikos am Beispiel der Wirtschaftsuniversität Wien}


Dieser Teil umfasst die empirische Analyse der Studiendauer und des Studienabbruch-Risikos auf Basis

- der in der Hörerevidenz verfügbaren Stamm-, Studien- und Prüfungsdaten von mehreren inländischen Immatrikulationsjahrgängen (der Wirtschaftsuniversität Wien) sowie der

- ergänzenden Daten aus einer Befragung der inländischen Immatrikulationsjahrgänge der Wintersemester 1990 und 1993.

Im Mittelpunkt dieses Teils steht die Anwendung der im ersten Teil beschriebenen Verfahren der Ereignisanalyse zur Ermittlung von Faktoren, die lange Studiendauern und hohe Studienabbruchraten erklären. Die Ergebnisse der empirischen Analysen zeigen, dass die Methoden der Ereignisanalyse die Beantwortung der den Zielsetzungen der Arbeit entsprechenden Fragestellungen (siehe Abschnitt 1.1) zu Studiendauer und -abbruch ermöglichen.

Weiters wird herausgearbeitet, welche Ergebnisse über Studiendauer und Studienabbruch-Wahrscheinlichkeit mit Hilfe der Daten aus der Hörerevidenz routinemäßig erzeugt werden können. Einige Fragen hingegen lassen sich nur durch Einbeziehung der Daten aus einer zusätzlich durchgeführten Befragung beantworten. 


\section{Kapitel 9}

\section{Studien zu den Themen Studiendauer und Studienabbruch}

Zur Untersuchung von Gründen und Ursachen für die hohen StudienabbruchQuoten und die langen Studienzeiten gibt es international und national viele Studien. Die meisten Studien basieren auf Befragungen von Studienabbrechern, Studierenden oder Absolventen. In diesem Kapitel wird insbesondere auf eine Studie der Österreichischen Hochschülerschaft (Grossmann et al. (1999a)) und auf Untersuchungen zu diesem Thema, die in den letzten Jahren an der Wirtschaftsuniversität durchgeführt worden sind, kurz eingegangen. Es werden die unterschiedlichen Ansätze, einerseits Befragungen und andererseits Analysen der Daten aus der Hörerevidenz, die verwendeten Verfahren und die Ergebnisse diskutiert. Diese Studien sollen einen ersten Aufschluss darüber geben, welche Faktoren einen Erklärungsbeitrag zum Studienabbruch und zu den langen Studiendauern leisten. Abschließend werden zwei (internationale) Arbeiten diskutiert, in denen die Dauer bis zum erfolgreichen Studienabschluss bzw. bis zum Studienabbruch mit Hilfe von Ereignisdatenmodellen analysiert wurde.

\subsection{Befragungen}

Die Österreichische Hochschülerschaft führte in Zusammenarbeit mit dem Institut für Statistik und Decision Support Systems der Universität Wien eine "Empirische Untersuchung über Gründe und Ursachen für die langen Studienzeiten in Österreich" durch (Grossmann et al. (1999a)). Befragt wurden aktive 
Studierende aus ganz Österreich, die sich vorwiegend im zweiten Studienabschnitt befanden. Die Befragung konzentrierte sich auf

1. die soziale Situation der Studierenden, primär auf die Finanzierung des Studiums durch

- Erwerbstätigkeit

- Studienbeihilfe

- Unterstützung durch Eltern/Familie

2. den Übertritt Schule-Universität

- Schulische Ausbildung

- Gründe für die Studienwahl

- Studieneingangsphase

- Orientierungslehrveranstaltungen

3. das Studium

- Organisatorische Probleme im Studienverlauf

- Zugang zur Information über das Studium

- Betreuung und Ausstattung

- Lehrinhalte und Lehrveranstaltung

4. die subjektive Einschätzung der Studiendauer

Die Analysen wurden sowohl für den gesamten Datensatz als auch für die Studiengruppen und Fakultäten getrennt durchgeführt. Für die vorliegende Arbeit von Interesse sind die Auswertungen für die Studiengruppe "Betriebswirtschaftslehre", die die Studienrichtungen Betriebswirtschaftslehre, Handelswissenschaften und Internationale Betriebswirtschaftslehre umfasst; siehe Grossmann et al. (1999b). Die einzelnen Variablen zu den oben angeführten Themen (wie etwa Geschlecht, Alter, durchschnittliche Berufsintensität in den ersten drei Semestern, Hauptaktivität Beruf oder Studium, Schultyp etc.) wurden in Häufigkeitstabellen für diese Studiengruppe und im Vergleich mit allen sonstigen Studien dargestellt. Potentielle Erklärungsfaktoren für die Überschreitung der Mindeststudiendauer wurden mit Hilfe von Kreuztabellen der erhobenen Variablen mit der Studiendauer analysiert. Für die Studiendauer verwendeten die Autoren die Dauer des ersten Abschnitts, die in die beiden Kategorien "Überschreitung der Mindeststudienzeit plus Toleranzsemester ja oder nein" unterteilt wurde und somit als dichotomes Merkmal in die Analyse einging. 
Erwähnenswerte Ergebnisse dieser (univariaten) Auswertungen (für die Studiengruppe Betriebswirtschaftslehre) sind etwa:

- Von den männlichen Befragten überschreiten die Mindeststudiendauer des ersten Abschnitts (plus Toleranzsemester) ca. 68\% und von den weiblichen Studierenden knapp $60 \%$.

- Ca. $85 \%$ der Studierenden, die in den ersten drei Semestern voll berufstätig waren, das sind $4.3 \%$ der Befragten, überschreiten die Mindeststudiendauer (plus ein Semester); bei den Nicht-Berufstätigen, $54 \%$ der Befragten, sind es hingegen nur $59 \%$.

- Studierende, die als ihre Hauptaktivität den Beruf angeben (17\%), überschreiten zu $73 \%$ die Mindestdauer (plus eine Semester) und Studierende mit Hauptaktivität Studium zu $62 \%$.

- Von den Studierenden, die Schwierigkeiten am Studienbeginn angeben, überschreiten etwa $77 \%$ die Mindeststudiendauer (plus ein Semester); bei den Studierenden ohne Schwierigkeiten am Studienbeginn liegt der Wert bei $54 \%$.

- Auch zwischen den verschiedenen Matura-Schultypen zeigen sich deutliche Unterschiede; so studieren $57 \%$ der BHS-Maturanten länger als die Mindeststudienzeit des ersten Abschnitts (plus Toleranzsemester), bei den AHSMaturanten sind es hingegen $72 \%$.

Diese Ergebnisse sind Hinweis auf potentielle Erklärungsfaktoren für lange Studienzeiten.

Eine Analyse zum Studienabbruch an der Wirtschaftsuniversität führten Schneider und Weiländer (1990) in Zusammenarbeit mit fünf Diplomanden für die Studierenden des Immatrikulationsjahrgangs 1986/87 durch. Die Autoren nützten die Daten aus der Hörerevidenz der WU für die ersten sechs Studiensemester, um zwischen folgenden Teilgruppen zu unterscheiden:

1. "Wenigscheinige" (Studienabbrecher mit höchstens fünf inskribierten Semestern bzw. im sechsten Semester noch inskribierte Studierende mit höchstens fünf Leistungsnachweisen)

2. Durchschnittsleister (keine Studienabbrecher, 9-13 Leistungsnachweise in fünf Semestern)

3. Hochleister (keine Studienabbrecher, 19-23 Leistungsnachweise in fünf Semestern) 
Im Anschluss an die deskriptive Analyse der Prüfungsdaten aus der Hörerevidenz (Bildung der relativen und absoluten Anzahl von Scheinen in den Semestern) wurde aus jeder Gruppe eine zufällige Stichprobe gezogen und (strukturierte) Interviews geführt. Das Hauptinteresse galt der Gruppe der Wenigscheinigen.

Der in den Interviews verwendete Fragebogen umfasste ähnliche Themen wie die angeführten Punkte der ÖH-Studie. Ergänzend wurden die Studienabbrecher auch nach den Abbruchgründen und -tätigkeiten gefragt und die "Performance" (Anwesenheit, Antritte) der Befragten in den einzelnen Pflichtlehrveranstaltungen des ersten Abschnitts untersucht. Die Autoren kommen beispielsweise zu dem Ergebnis, dass Studienabbrecher zu einem sehr frühen Zeitpunkt aus dem Universitätssystem ausscheiden. Sie haben zu diesem Zeitpunkt kaum Leistungsnachweise erbracht, jedoch meist mehrmals versucht, eine Lehrveranstaltung zu absolvieren. Als wichtigste Abbruchgründe werden angeführt:

- Studium entspricht nicht den Vorstellungen und Neigungen

- Konzentration auf ein anderes Studium

- Günstiges berufliches Angebot

- Doppelbelastung durch Erwerbstätigkeit

Die vorwiegende Tätigkeit nach dem Abbruch wird (in Reihenfolge der Häufigkeit) wie folgt angegeben:

- Erwerbstätigkeit

- Konzentration auf ein anderes Studium

- Konzentration auf eine andere Ausbildung

Ergebnisse aus den fünf Diplomarbeiten, die im Zuge dieser Studie durchgeführt worden sind, werden zusammenfassend von Häfke-Schönthaler (1999) erläutert.

In Deutschland führt das Hochschulinformationssystem (HIS) zum Thema Studienablauf Längsschnittbefragungen von Hochschulabsolventen durch. Es werden Daten erhoben, die von der Prüfungsstatistik nicht bereitgestellt werden können und die zur Analyse von Studienverlaufs- und -erfolgsdaten wichtig sind. Schwerpunkte der Untersuchung sind die Bereitstellung von Information struktureller Daten zum Studienverlauf wie 
- Fach- und Hochschulwechsel

- Studienunterbrechungen

- Auslandsstudium

- Studienbedingungen

- Berufstätigkeit

\subsection{Analyse der Daten aus der Hörerevidenz}

Eine Studienverlaufsanalyse an der Wirtschaftsuniversität, die ausschließlich auf Basis der Daten aus der Hörerevidenz durchgeführt worden ist, ist die Arbeit von Hoffmann (1987). Die retrospektive Vorstudie ausgehend von den Absolventen des Studienjahres 1985/86 wurde zur Ermittlung der Verteilung der Studiendauer - ergänzend getrennt nach Geschlecht und Studienrichtung - durchgeführt. Beispielsweise wurde für diesen Absolventenjahrgang eine (retrospektive) durchschnittliche Studiendauer von 12.3 und ein Median von 12 Semester ermittelt. Die prospektive Hauptstudie beinhaltet die Untersuchung der Studienanfänger des Wintersemesters 1979/80, deren Studienverlauf bis zum Herbst 1986 beobachtet worden ist. Mit dem Stichtag wurden vier Gruppen von Studienanfängern unterschieden:

1. Studierende, die bis zum Stichtag noch keine Teildiplomprüfung abgelegt haben. Die Studienpläne der Wirtschaftsuniversität werden beispielsweise auf den Internet-Seiten der Wirtschaftsuniversität ausführlich erläutert (http://www.wu-wien.ac.at/stab); grob kann gesagt werden, dass in jedem Studienabschnitt einige große Prüfungen, die sogenannten Vor- bzw. Teildiplomprüfungen absolviert werden müssen, zu denen man nur antreten kann, wenn vorher dazugehörige Lehrveranstaltungsprüfungen (sogenannte "Scheine") abgelegt worden sind.

2. Studierende, die schon eine Teildiplomprüfung abgelegt haben, aber sich zum Stichtag noch im ersten Abschnitt befinden

3. Studierende im zweiten Abschnitt

4. Absolventen

Die prospektive Analyse umfasst deskriptive Analysen wie Häufigkeitsverteilungen der Grundgesamtheit nach Studiendauer (gesamt sowie getrennt nach 
dem ersten und zweiten Studienabschnitt), Geschlecht, Alter bei Studienbeginn, Schultyp sowie dem Ablegesemester und der Anzahl der Antritte zu den jeweiligen Vor- und Teildiplomprüfungen des ersten und zweiten Studienabschnitts. Mit Hilfe von $\chi^{2}$-Tests und Varianzanalysen wurden auch Untersuchungen zur Abhängigkeit und Prognosemöglichkeit des Studienerfolgs mit den im System vorhandenen Variablen wie Geschlecht, Nationalität, Schultyp und Alter durchgeführt. Als Indikator für den Studienerfolg wurde der Studierstatus und die Studiendauer verwendet. Der Autor kommt zu dem Schluss, dass die einbezogenen Variablen die Studiendauer zwar beeinflussen, aber nur zu einem geringen Teil erklären. Eine (Individual)Prognose der Studiendauer zu Beginn des Studiums mit den im System verfügbaren Variablen sei daher nicht sinnvoll. Ergänzend erwähnt der Autor in Anlehnung an andere zum Thema durchgeführte Projekte, dass alleine durch die Miteinbeziehung der Prüfungsaktivitäten im ersten Studienjahr (Anzahl, Noten) der Studienerfolg mit hoher Sicherheit prognostizierbar sei.

Eine aktuellere Studie auf Basis der Daten aus der Hörerevidenz ist die Arbeit von Häfke-Schönthaler (1999). Im Mittelpunkt dieser Arbeit stehen die Leistungen der Studienanfänger im ersten Jahr und deren Einfluss auf die Studiendauer und die Wahrscheinlichkeit des Studienabbruchs. Zielgruppe der Untersuchung sind alle inländischen, ordentlichen Hörer, die in den Wintersemestern 1990/91 - 1993/94 erstmals an der Wirtschaftsuniversität ein Diplomstudium der aktuellen Studienordnung inskribiert haben. Diese Jahrgänge wurden bis zum Stichtag 1. Juli 1996 beobachtet. Die Analysen wurden getrennt durchgeführt für "aktive" (zumindest einen Leistungsnachweis im ersten Studienjahr) und "passive" Studierende sowie für Erstimmatrikulierte und jene Erstinskribenten, die an einer anderen Universität oder schon früher an der Wirtschaftsuniversität immatrikuliert haben, aber erst im betrachteten Wintersemester erstmals ein ordentliches Diplomstudium (der aktuellen Studienordnung) inskribiert haben. Neben Häufigkeitstabellen für die Anzahl der (positiven) Leistungen im ersten Studienjahr und für die im System vorhandenen Variablen wie Geschlecht, Alter und Schulbildung untersucht die Autorin mit Hilfe der logistischen Regression den Einfluss dieser Faktoren auf den Studienerfolg und die Studienabbruch-Wahrscheinlichkeit. Zusammenfassend kommt die Autorin zu dem Schluss, dass die Leistungen im ersten Studienjahr einen großen Einfluss auf den Studienerfolg haben und dass nicht Misserfolge bei Prüfungen sondern Aktivität bzw. Inaktivität ein guter Prädiktor für den Studienabbruch ist.

Eine ausführliche Arbeit über retrospektive Analysen von Absolventenjahrgängen mit Hilfe der zur Verfügung stehenden Hörerevidenzdaten (für den Fachbereich Wirtschaftswissenschaften der Johann Goethe-Universität in Frankfurt 
am Main) präsentiert Würger (1992). Als Grundgesamtheit wählte der Autor die Absolventen von zehn Prüfungsterminen der drei Studienrichtungen BWL, VWL und WIP zwischen Sommersemester 1984 und Wintersemester 1988/89. Er teilt das Studium in mehrere Phasen und untersucht Beziehungen zwischen

- den Prüfungsresultaten des Studiums,

- der Studiendauer und dem Prüfungserfolg und

- den einzelnen Studienphasen zueinander.

Die Beziehungen wurden ergänzend getrennt nach Geschlecht, Nationalität, Bildungsstatus und Studienrichtung analysiert.

Zum Abschluss dieses Abschnitts wird noch auf eine aktuelle Arbeit aus England eingegangen. Die Autoren Smith und Naylor (2001) analysieren in ihrem Artikel die Studienabbruch-Wahrscheinlichkeiten jener Studienkohorte, die im Studienjahr 1990/91 in Großbritannien ein dreijähriges Studium begonnen hat. Als Motivation für ihre Arbeit nennen die Autoren

- die Einführung von Leistungskennzahlen (engl. performance indicators) für die Universitäten in Großbritannien, die die Abbruchquote neben dem Studienfortschritt als einen dieser Indikatoren enthalten. Aktuelle Details zu den Leistungskennzahlen in Großbritannien können auf den Internet-Seiten des HEFCE (Higher Education Funding Council for England) nachgelesen werden (http://www.hefce.ac.uk). Ziel der Autoren ist es, Einflussgrößen auf die Wahrscheinlichkeit des Studienabbruchs zu ermitteln und zu erklären, inwieweit die einbezogenen Variablen die Unterschiede in den Abbruchquoten verschiedener Universitäten und Studienrichtungen erklären;

- die jüngsten Kürzungen in den Förderungen von Studierenden aus ärmeren Verhältnissen; es wird vermutet, dass sich diese Kürzungen negativ auf die Anzahl der Studienanfänger und auf die Abbruchquote von Studierenden aus ärmlicheren Verhältnissen auswirken werden. Daher analysieren die Autoren auch den Einfluss des sozialen Status auf die Abbruchwahrscheinlichkeit;

- das Interesse der Universitäten und Studierenden selbst an Erklärungsfaktoren für den Studienabbruch.

Um die Studienabbruch-Wahrscheinlichkeit in Abhängigkeit von erklärenden Variablen zu schätzen, wurde ein Probit Modell (siehe zum Beispiel Aldrich 
und Nelson (1987)), getrennt nach Geschlecht, implementiert. Die Daten zum Studienabbruch und die in das Modell einbezogenen erklärenden Variablen entstammen einer Datenbank, in der die Daten aus der UK-weiten Studenten(Universities Statistical Records) und Schul-Datenbank zusammengeführt werden. Diese Datenbank enthält

- persönliche Informationen: Geburtsdatum, Geschlecht, Familienstand, Heimatadresse, Beschäftigung der Eltern;

- Informationen zur akademischen Vergangenheit: weitere akademische Ausbildungen, a-priori Qualifikationen;

- Studiendaten;

- Studienabbrecher-Informationen: erreichte Qualifikation, Abbruchgrund, Hauptaktivität nach dem Abbruch;

- Schuldaten: Schultyp, Noten.

Die Autoren kommen zu dem Ergebnis, dass die Studienabbruch-Wahrscheinlichkeiten mit den individuellen Charakteristiken variieren und niedriger für Studierende mit besseren Schulnoten, staatlicher Schulausbildung und besserem sozialen Hintergrund sind. Jedoch gibt es auch nach Einbeziehung der zahlreichen erklärenden Variablen beträchtliche Unterschiede in den Abbruchquoten zwischen den Universitäten und Studienrichtungen.

Die Studie ist vor allem deshalb von Interesse, weil sie

- mit einem umfassenden Datenmaterial durchgeführt worden ist, das auf die Studenten- und Schuldaten von Großbritannien zurückgreift, und

- aufzuzeigen versucht, dass nicht alleine die „nackten” Abbruchquoten als Leistungsindikatoren für einen Vergleich von Universitäten herangezogen werden können, sondern zumindest die unterschiedlichen Eingangsvoraussetzungen der Studierenden der verschiedenen Universitäten und Studienrichtungen berücksichtigt werden müssen.

\subsection{Studien unter Verwendung der Ereignis- datenanalyse}

In diesem Abschnitt wird auf zwei internationale Arbeiten eingegangen, in denen zur Analyse der Studiendauer und des Studienabbruch-Risikos para- 
metrische und semiparametrische Ereignisdatenmodelle implementiert worden sind.

Booth und Satchell (1995) analysieren in ihrem Artikel die Determinanten des Studienabbruchs und des erfolgreichen Studienabschlusses von britischen Doktoratsstudenten, die $1980 \mathrm{ihr}$ Diplomstudium abgeschlossen haben und bis 1986 ein Doktoratsstudium begonnen haben. Für die statistische Analyse der (aus einer Befragung) verfügbaren Daten implementierten sie ein Mehr-ZustandsModell mit den zwei End-Zuständen "Studium abgeschlossen" und "Studium abgebrochen" (siehe Kapitel 7). Studierende, die zum Stichtag im Jahr 1986 das Studium weder abgeschlossen noch beendet hatten, wurden als rechtszensierte Fälle in die Studie aufgenommen.

Als erklärende Variablen nahmen die Autoren folgende zeitkonstante Variablen in das Modell auf, gemessen am Studienbeginn: Form der finanziellen Unterstützung, Vollzeit- oder Teilzeitstudent zu Studienbeginn, StudienabschlussBeurteilung (degree class), Schulnoten (A level scores), Diplomstudienrichtung, Doktoratsstudienrichtung, Familienstand, Kinder, sozialer Status sowie Informationen über den Erwerb eines Masters oder einer anderen - nach Beendigung des Diplomstudiums - erworbenen Qualifikation. Da keine Informationen über einen Wechsel von Vollzeit- zu Teilzeitstudent oder umgekehrt verfügbar waren, konnte diese Variable nicht als zeitabhängige Variable integriert werden. Bei der Interpretation ist daher zu beachten, dass diese Variable nur etwas über den Zustand zu Studienbeginn aussagt.

Die Daten wurden getrennt nach dem Geschlecht analysiert; als parametrisches Regressionsmodell zur Analyse der Studienabschluss- und -abbruchraten wurde das verallgemeinerte Gamma-Modell implementiert und in Folge die Hypothese $\kappa=1$ zur Überprüfung der Annahme eines Weibull-Modells getestet (siehe Abschnitt 5.1). Neben dem Effekt der einbezogenen erklärenden Variablen wurden auch die (bedingte) erwartete Studiendauer bis zum Abschluss und bis zum Abbruch sowie die Abschluss- und die Abbruchwahrscheinlichkeiten für unterschiedliche Ausprägungen der Variablen geschätzt.

Die Autoren kommen zusammenfassend zu dem Ergebnis, dass die Studienabschlussraten zwischen den verschiedenen Doktoratsstudienrichtungen - unter Kontrolle der anderen einbezogenen Variablen - stark differieren, für naturwissenschaftliche Studienrichtungen besonders hoch und für Sprachen und Kunst besonders niedrig sind. Weiters hat der Status "Teilzeitstudent" zu Studienbeginn einen negativen Effekt auf die Studienabschlussrate.

Yamaguchi (1991) analysiert als Anwendung eines Cox-Modells mit zeitabhängigen Kovariablen (siehe Kapitel 6) die Abbruchraten einer Kohorte von Stu- 
dienanfängern. Als zeitunabhängige erklärende Variablen bezieht der Autor das Geschlecht, Schulnoten, den Studieneintritt als Vollzeit- oder Teilzeitstudent und den Zeitabstand zwischen Schulabschluss und Studienbeginn in das Modell ein. Der Familienstand (verheiratet oder nicht) und die Berufstätigkeit (als dichotomes Merkmal: weniger als oder mindestens 20 Stunden beschäftigt) wurden als zeitabhängige (endogene) Variablen modelliert. Yamaguchi kommt zu dem Schluss, dass verheiratete Studenten, Teilzeitstudenten zu Studienbeginn und berufstätige Studierende Abbruch-gefährdeter sind. Gute Noten in der Schule und ein geringerer Zeitabstand zwischen Schule und Studium verringern das Risiko eines Studienabbruchs. 


\section{Kapitel 10}

\section{Daten und erklärende Variablen}

Die Datenbanken von Universitäten verfügen über ein umfangreiches Datenmaterial zu ihren Studierenden. Im vorherigen Kapitel sind einige Analysen zum Thema Studiendauer und -abbruch diskutiert worden, die mit Hilfe der in diesen Datenbanken verfügbaren Daten durchgeführt worden sind. Zumeist ist die Ermittlung von Einflussfaktoren und die Prognostizierbarkeit des Studien(miss)erfolgs (der Studiendauer bzw. der Studienabbruch-Wahrscheinlichkeit) in Abhängigkeit von den aus den Daten der Hörerevidenz ermittelten Einflussfaktoren ein zentrales Ziel der Analysen. Auch in der vorliegenden Arbeit werden die in den Verwaltungssystemen der Wirtschaftsuniversität verfügbaren Hörerevidenz-Daten dazu genützt, um das eben angeführte Ziel zu verfolgen.

Von der zentralen Verwaltung wurden im Jänner 2001 für die Zwecke des (zu Beginn dieser Arbeit erwähnten) Projekts "Studienverlaufsanalyse" (siehe Hackl und Sedlacek (2002)) alle Stammdaten, Diplomstudiendaten und (bis dahin erzielten) Prüfungsergebnisse (in Diplomstudien) von Studierenden, die vor dem Wintersemester 1996/97 in Österreich immatrikuliert haben, aus der Studenten-Datenbank (STEP) herausgeladen und als ASCII-Dateien zur Verfügung gestellt. Als Stichtag (= Ende des Beobachtungszeitraums) der Studie wird der 31. Dezember 2000 definiert; alle Aussagen der Studie beziehen sich insoferne auf diesen Zeitpunkt, als die gesamte Information in den Ergebnissen berücksichtigt ist, die die Vergangenheit vor dem Stichtag beschreibt. Die Daten von Studierenden, die nach dem Wintersemester 1996/97 in Österreich immatrikuliert haben, wurden in das Projekt nicht einbezogen, weil unter diesen Studierenden bis zum Stichtag keine Absolventen zu erwarten waren und daher keine Aussagen über die Studiendauer bis zum erfolgreichen Abschluss gemacht werden können. Diese Arbeit greift insbesondere auf die Daten der Immatrikulationsjahrgänge 1989 bis 1993 zurück. 
In diesem Kapitel wird im ersten Abschnitt erläutert, welche Daten von Studierenden in der Hörerevidenz verfügbar sind und in welcher Form sie für die empirischen Analysen des Studienerfolgs verwendet werden können. Ein Schwerpunkt des ersten Abschnitts liegt in der Definition der Dauer bis zum erfolgreichen Studienabschluss bzw. bis zum Studienabbruch und den in diesem Zusammenhang auftretenden Problemen.

Analysen, die ausschließlich auf Basis von Daten aus der Hörerevidenz durchgeführt werden, ist gemeinsam, dass potentielle Erklärungsgrößen aus dem (sozialen) Umfeld der Studierenden wie die Berufstätigkeit oder eine zweite Ausbildung neben dem Studium nicht in das Modell einbezogen werden können, da sie im System nicht verfügbar sind. Daher wurden weitere Daten in die Studie einbezogen, die in einer im Rahmen der Studie durchgeführten Befragung von (ehemaligen) Studierenden erhoben wurden. Das Design der Befragung, die erhobenen Variablen und die Zusammenführung dieser Daten mit den Daten aus der Hörerevidenz sind das Thema des zweiten Abschnitts.

\subsection{Daten aus der Hörerevidenz (Systemda- ten)}

Die Daten aus der Hörerevidenz werden in Stamm-, Studien- und Prüfungsdaten unterschieden.

\section{Stammdaten}

Für jeden Studierenden der Wirtschaftsuniversität sind folgende Stammdaten in der Hörerevidenz verfügbar:

- Matrikelnummer (MaNr)

- Geburtsdatum

- Geschlecht

- Nationalität

- Heimat- und Zustelladresse; die Adressen können sich während der Studienzeit ändern, im System sind nur die aktuellen Adressen - sofern die Änderungen vom Studierenden bekannt gegeben werden - eingetragen 
- Datum der Reifeprüfung

- Schulform, in der die Reifeprüfung abgelegt worden ist; hier werden 33 Ausprägungen unterschieden; siehe Anhang A.

Mit Hilfe dieser Stammdaten wurden folgende Variablen für die in dieser Arbeit durchgeführten empirischen Analysen definiert:

- Alter bei Studienbeginn (in Jahren)

- Alter zum Zeitpunkt der Matura

- Geschlecht

- Nationalität

- Zeit zwischen Matura und Studienbeginn

- Schultyp; für diese Variable wurden aus den vorhandenen Ausprägungen (siehe Anhang A) folgende Kategorien gebildet:

- Handelsakademie (HAK); entspricht der Schulform 20

- Gymnasium (AHS); für diese Kategorie wurden die Schulformen 1 bis 10 und 12 bis 16 der im System verfügbaren Ausprägungen zusammengefasst

- Höhere Technische Lehranstalt (HTL); hierfür wurden die Kategorien 19 und 23 zusammengefasst

- Höhere Lehranstalt für wirtschaftliche Berufe (HLA); Ausprägungen 11 und 21

- Sonstige (alle anderen Ausprägungen)

Für empirische Analysen der Studiendauer und des Studienabbruch-Risikos empfiehlt es sich, die Studierenden zu unterscheiden in

- inländische Immatrikulierte, das sind Studierende, die an der Wirtschaftsuniversität immatrikuliert haben und im Immatrikulationssemester ein ordentliches Diplomstudium inskribiert haben, und in

- Zusatzstudenten, das sind Studierende, die an der Wirtschaftsuniversität ein ordentliches Diplomstudium inskribiert haben und

- ausländischer Nationalität sind, 
- inländischer Nationalität sind und an einer anderen österreichischen Universität immatrikuliert haben,

- inländischer Nationalität sind und an der WU immatrikuliert haben, aber nicht im Immatrikulationssemester ein ordentliches Diplomstudium eröffnet haben.

Die Anzahl dieser Zusatzstudierenden ist in den letzten Jahren stark gestiegen. Etwa ein Viertel aller Absolventen des Studienjahres 1999/2000 und etwa 50\% der Erstinskribenten des Wintersemesters 1996/97 zählen zu dieser Kategorie. Es empfiehlt sich, die Zusatzstudierenden separat zu behandeln, weil

- aus vorangegangenen Studien (siehe etwa Häfke-Schönthaler (1999)) bekannt ist, dass sie häufig ein anderes Studierverhalten als die inländischen Immatrikulierten zeigen (Doppelstudenten, Schnupperstudenten, Erwerb von Zusatzqualifikation zum eigentlichen Hauptstudium); dieser Punkt kommt insbesondere bei Analysen von Studieneingangs-Kohorten zum Tragen.

- ihre WU-Studiendauer in einigen Fällen aufgrund von Anrechnungen des vorhergehenden (verwandten) Studiums bzw. aus dem Ausland nach unten verzerrt ist.

Der Schwerpunkt der Analysen in dem vorliegenden Bericht liegt auf den Daten von inländischen Immatrikulierten. Einige Kennzahlen zu den Zusatzstudierenden können in Hackl und Sedlacek (2002) nachgelesen werden.

\section{Diplomstudiendaten}

Die Studierenden der WU konnten nach dem bis zum Wintersemester 2002/03 gültigen (und daher dieser Arbeit zugrunde liegenden) Studienplan unter vier ordentlichen Diplomstudien wählen, wobei sie häufig mehr als eine Studienrichtung inskribierten. Beispielsweise haben etwa $75 \%$ der Absolventen des Studienjahres 1999/2000 mehr als eine Studienrichtung (bzw. Studienordnung) inskribiert. Für jedes an der Wirtschaftsuniversität inskribierte ordentliche Diplomstudium liegen in der Studentendatenbank STEP zu jeder Matrikelnummer folgende Systemdaten vor:

- Kennzahl der Studienrichtung (Betriebswirtschaftslehre (BW), Handelswissenschaften (HW), Wirtschaftspädagogik (WIPÄD), Volkswirtschaftslehre (VW)) 
- Datum des Studienbeginns

- Ende des ersten Studienabschnitts (das ist das Datum des Bestehens der letzten Teildiplomprüfung im ersten Abschnitt)

- Ende des zweiten Studienabschnitts (das ist das Datum des erfolgreichen Studienabschlusses und gleichbedeutend mit dem Datum des Bestehens der letzten Teildiplomprüfung im zweiten Abschnitt)

- Datum, an dem das Studium geschlossen worden ist; dieses Datum entspricht

- dem Ende des zweiten Studienabschnitts bei erfolgreichem Abschluss des Studiums,

- dem Datum vom Ende des zweiten von drei nicht inskribierten Semestern in Folge (Schließung durch die Universität) oder

- dem Datum der selbst veranlassten Schließung des Studiums.

Der Eintrag wird in den beiden letzten Fällen gelöscht, falls das Studium wieder aufgenommen wird.

- Anzahl der inskribierten Semester

- eine Variable, die angibt, ob der Erfolgsnachweis aus Buchhaltung und Kostenrechnung mit Studienbeginn erbracht ist oder nicht; die meisten Studierenden erbringen diesen Erfolgsnachweis zu Studienbeginn nicht und müssen ihn in Form von Ergänzungsprüfungen an der Wirtschaftsuniversität selbst erbringen; eine Ausnahme bilden etwa Absolventen der Handelsakademie.

Die Diplomstudiendaten werden zur Ermittlung der

- Dauer bis zum erfolgreichen Studienabschluss (Studiendauer)

- Dauer bis zum Abschluss des ersten Studienabschnitts

- Dauer bis zum Studienabbruch

- Dauer bis zum Stichtag (bei noch im Gang befindlichen Studien)

benötigt. Studienverlaufsanalysen erfordern eine genaue Definition dieser Begriffe. In der vorliegenden Arbeit wird die Studiendauer bis zum erfolgreichen Abschluss definiert als: 
(a) Die Studiendauer ist der Zeitraum zwischen dem Datum des Studienbeginns an der WU und dem des (ersten) Abschlusses eines WU-Studiums. Je nach Modellierung kann diese Zeit exakt oder in Semestern angegeben werden.

Für diese Definition ergeben sich folgende Vorteile:

- Einfache Berechnungsweise mit Hilfe der Systemdaten

- Die Definition berücksichtigt, dass Studienrichtungen an der WU (insbesondere die am häufigsten gewählten Studienrichtungen Betriebswirtschaftslehre und Handelswissenschaften; ca. 90\% der WU-Absolventen haben eines dieser beiden Studien absolviert) einander sehr ähnlich sind und daher der überwiegende Teil von Lehrveranstaltungen bei einem Studienrichtungswechsel anrechenbar ist. Definiert man als Studiendauer etwa nur die Verweildauer im abgeschlossenen Diplomstudium, wird die eigentliche echte Studiendauer bei einem Studienrichtungswechsel unterschätzt.

Unter den an der WU immatrikulierten inländischen Absolventen, die im Studienjahr 1999/2000 (Absolvierung der zweiten Diplomprüfung zwischen 1.10.1999 und 30.9.2000) an der WU ihr erstes Diplomstudium abgeschlossen haben, finden sich beispielsweise ca. 15\%, bei denen der Studienbeginn des abgeschlossenen Studiums mindestens ein Semester (und im Durchschnitt 4 Semester) nach dem Studienbeginn an der WU liegt.

Ein Nachteil dieser Definition ist:

- Jede Studienunterbrechung - ob offiziell (keine Inskription), inoffiziell (Inskription, aber keine Zeitinvestition in das Studium) oder Exmatrikulation und Wiederaufnahme des WU-Studiums - wird als Studienzeit mitgerechnet. In Analysen von mehreren Absolventenjahrgängen konnte gezeigt werden, dass nur etwa $4 \%$ aller Absolventen während ihrer Studienzeit mindestens ein und durchschnittlich 2.5 Semester nicht inskribiert haben; hiervon zumeist Studierende, die sehr lange für ihr Studium gebraucht haben.

Dieselbe Definition kann analog auch für die Dauer des ersten Studienabschnitts verwendet werden. Alternative Definitionen für die Studiendauer, ihre Vor- und Nachteile sowie empirische Vergleiche sind in Hackl und Sedlacek (2002) nachzulesen.

Als Dauer bis zum Studienabbruch wird sinngemäß die Definition

(a) Zeit zwischen Immatrikulation und Studienabbruch eingeführt.

Hierfür wird eine exakte Definition des Ereignisses Studienabbruch benötigt. Wir diskutieren im folgenden zwei alternative Definitionen. 
(A) Unter System-Abbruch (zu einem Zeitpunkt) wird die (nicht abschlussbedingte) Schließung aller an der Wirtschaftsuniversität inskribierten ordentlichen Diplomstudien verstanden.

System-Abbrecher sind somit Studierende, die vor dem Stichtag alle ihre an der Wirtschaftsuniversität begonnenen Studien abgebrochen haben. Studierende, die etwa das Studium der Handelswissenschaften "abbrechen" und das der Betriebswirtschaftslehre an der Wirtschaftsuniversität fortsetzen und abschließen, werden nicht als Abbrecher des WU-Studiums behandelt, sondern als Absolventen (des BW-Studiums). Hingegen werden Studierende, die ihr Studium an der Wirtschaftsuniversität abgebrochen haben und an einer anderen Universität ein anderes (eventuell auch wirtschaftswissenschaftliches) Studium fortgesetzt (und möglicherweise abgeschlossen) haben, als Studienabbrecher behandelt. Ihr erfolgreicher Studienabschluss an einer anderen Universität wird nicht berücksichtigt. Es ist zu beachten, dass System-Abbrecher (zum Stichtag) ihr WU-Studium zu einem späteren Zeitpunkt wieder aufnehmen können und dann nicht mehr zu den System-Abbrechern zählen.

Grundsätzlich gelten für die Ermittlung der Dauer bis zum Studienabbruch nach Definition (a) die bereits im Zusammenhang mit dem Studienabschluss diskutierten Vor- und Nachteile, allerdings müssen noch weitere Probleme beachtet werden.

Da Studienabbrecher häufiger als Absolventen ihr Studium "offiziell" unterbrechen (mindestens ein Semester nicht inskribieren), wird die Dauer bis zum Abbruch mehr als bei den Absolventen überschätzt. Wird das Datum des Studienabbruchs, zu dem das Studium (die Studien) geschlossen wurde(n), unter Verwendung der im System vermerkten Daten ermittelt, wird die tatsächliche Dauer bis zum Abbruch meist deutlich überschätzt (und die Anzahl der Studienabbrecher eines Studienanfänger-Jahrgangs zum Stichtag unterschätzt): Viele Studierende brechen ihr Studium ab, ohne dies der Evidenzstelle bekannt zu geben; das Studium wird jedoch - wie erwähnt - von offizieller Seite erst zu Beginn des dritten nicht inskribierten Semesters in Folge geschlossen. Studierende, die zum Stichtag schon abgebrochen haben aber noch nicht drei Semester in Folge nicht inskribiert haben, werden daher im System nicht als Studienabbrecher geführt. Zur Korrektur diees Fehlers wurde im Zuge der Studie eine alternative Definition der Begriffe Studienabbruch und Abbruchdatum überlegt.

(B) Studienabbruch liegt zusätzlich vor, wenn ein Studierender zum Stichtag vier Semester lang keine (positive oder negative) Leistung mehr erbracht und insgesamt weniger als zehn positive Vor- bzw. Teildiplomprüfungen abgelegt hat - unabhängig von der Zahl der inskribierten Semester in dieser Zeit. 
Diese Definition basiert auf der Überlegung, dass die Nicht-Aktivität im Studium meist ein Indikator für den Studienabbruch ist. Solche Studierenden werden in der Folge als nicht-aktive Studierende bezeichnet. Alle anderen Studenten werden als aktive Studierende geführt. Von Studierenden, die zumindest vier Semester lang inaktiv waren aber mindestens zehn positive Vor- bzw. Teildiplomprüfungen absolviert haben, kann vermutet werden, dass sie noch vorhaben, ihr Studium abzuschließen und beispielsweise an ihrer Diplomarbeit arbeiten.

Als Studienabbrecher werden in der Folge sowohl die System-Abbrecher (A) als auch die nicht-aktiven Studierenden (B) untersucht. Als Dauer bis zum Studienabbruch wird in dieser Arbeit zumeist die folgende Definition (b) verwendet:

(b) Die Dauer bis zum Studienabbruch beträgt von Studienabbrechern, die an der Wirtschaftsuniversität keine einzige Leistung erbracht haben, ein Semester; bei anderen Studienabbrechern entspricht sie der Zeit zwischen Studienbeginn und dem "Datum der letzten Leistung plus ein Semester".

Die entsprechenden Werte können aus den Daten der Hörerevidenz ermittelt werden.

Die eben angesprochenen Punkte zeigen, wie schwierig der Begriff Studienabbruch zu handhaben sind. Bei WU-Studienabbrechern kann es sich um erfolgreiche Universitätsabsolventen handeln, die ihr an der Wirtschaftsuniversität begonnenes Studium an einer anderen Universität fortgesetzt und abgeschlossen haben. Da der Wirtschaftsuniversität die weiteren Karrierewege der Studienabbrecher nicht bekannt sind, konnten diese bei der Modellierung des Studienabbruchs nicht berücksichtigt werden. Eine (derzeit nicht bestehende) Datenbank, die die Studenten-Daten aller österreichischen Universitäten enthält, würde eine detailliertere Analyse der Studienverläufe österreichischer Studenten ermöglichen; vgl. Smith und Naylor (2001).

\section{Prüfungsdaten}

Vor- und Diplomprüfungsdaten informieren zu jeder an der WU absolvierten bzw. angerechneten Vor- bzw. Diplomprüfung über

- die Studienrichtung, in der sie abgelegt worden ist

- das Datum der Prüfung

- die Note 
- die Zahl der Prüfungswiederholung (in der jeweiligen Studienrichtung)

- die Prüfer

- die Anrechnung (ja/nein); falls ja, sind bei Anrechnungen von Prüfungen, die nicht an der Wirtschaftsuniversität absolviert worden sind, die obigen Informationen meist nicht verfügbar.

Lehrveranstaltungsdaten informieren zu jeder an der WU absolvierten bzw. angerechneten Lehrveranstaltungsprüfung (sogenannte Scheine) über

- die Studienrichtung

- das Datum der Prüfung

- die Note

- die Prüfer

- die Anrechnung (ja/nein)

Die Prüfungsdaten wurden verwendet, um insbesondere folgende (für die durchgeführten empirischen Analysen bedeutenden) Variablen zu bilden:

- Anzahl aller an der WU abgelegten (positiven) Lehrveranstaltungsprüfungen im ersten Studienjahr

- Notendurchschnitt dieser Prüfungen; hierfür zählt nur die letzt gültige Note jeder Lehrveranstaltung

- Anzahl der an der WU abgelegten (positiven) Lehrveranstaltungsprüfungen zum Stichtag

- Anzahl der an der WU abgelegten (positiven) Vor- bzw. Diplomprüfungen im ersten Studienjahr

- Anzahl der an der WU abgelegten (positiven) Vor- bzw. Diplomprüfungen zum Stichtag

Lehrveranstaltungen, die nicht mit einer Beurteilung abgeschlossen worden sind (wie das Orientierungstutorium/OTU), wurden genauso wenig wie nicht an der Wirtschaftsuniversität abgelegte Prüfungen berücksichtigt. An der Wirtschaftsuniversität abgelegte Ergänzungsprüfungen, das sind Prüfungen aus Buchhaltung und Rechnungswesen, die jene Immatrikulierten ablegen müssen, 
die zu Studienbeginn keinen Nachweis dafür erbringen konnten, wurden grundsätzlich zu den abgelegten Lehrveranstaltungsprüfungen dazu gezählt. Mit Ausnahme der HAK-Absolventen müssen diese Ergänzungsprüfungen fast alle anderen Studienanfänger ablegen. Näheres zu den Studienplänen und zur Prüfungsordnung an der WU kann auf der Internet-Startseite der WU nachgelesen werden (http://www.wu-wien.ac.at).

\subsection{Ergänzende Daten aus einer Befragung}

Als vorrangiges Ziel der vorliegenden Arbeit wurde im ersten Kapitel die Ermittlung von Einflussfaktoren auf die Studiendauer und das StudienabbruchRisiko genannt. In der Hörerevidenz sind nur einige wenige Merkmale vorhanden, die potentielle Erklärungsfaktoren der beiden Größen sind. Variablen, von denen vermutet werden kann, dass sie die Studiendauer oder das Studienabbruch-Risiko beeinflussen, müssen ergänzend erhoben werden. Im ersten Teil dieser Arbeit ist diskutiert worden, dass das Weglassen potentieller Einflussgrößen zu Verzerrungen der Parameterschätzer der einbezogenen Variablen führen kann.

Beispielsweise besteht ein hohes Interesse der Universitätsleitung, aktuelle und detaillierte Aussagen zum Studienverhalten und -erfolg von berufstätigen und nicht-berufstätigen Studierenden der Wirtschaftsuniversität zu erhalten. Da die Berufstätigkeit zusätzlich zu den in der Hörerevidenz zur Verfügung stehenden Variablen als potentieller Einflussfaktor auf die Studiendauer und das Studienabbruch-Risiko vermutet wird, wurde eine Befragung zweier WU-Immatrikulationsjahrgänge zu Berufstätigkeit und anderen Faktoren des studentischen Umfeldes durchgeführt.

\section{Design der Befragung}

Es ist eine Stichprobe inländischer Immatrikulierter der beiden Wintersemester 1990/91 und 1993/94 gezogen worden. Zwei Jahrgänge wurden gewählt, um Vergleiche zwischen den beiden Kohorten ziehen zu können und um bei einer schriftlichen Befragung eine genügend große Stichprobe zu erhalten. Die Auswahl der beiden Jahrgänge begründet sich in der Möglichkeit, die Studierenden sehr lange verfolgen zu können. Zusatzstudenten blieben aus den in Abschnitt 10.1 genannten Gründen unberücksichtigt.

Die beiden Jahrgänge wurden (mit Stichtag August 2000) in die drei Schichten System-Abbrecher, Absolventen und Studierende unterteilt. Unter den 
(zufällig aus der Grundgesamtheit ausgewählten) System-Abbrechern und Absolventen wurde die Befragung telefonisch durchgeführt, da das in der Hörerevidenz gespeicherte Adressenmaterial für diese Gruppen nicht aktuell ist und deshalb bei einer postalischen Erhebung eine sehr geringe Rücklaufquote und stark verzerrte Ergebnisse erwartet wurden. Diese Vermutung ist durch eine postalische Pilot-Erhebung verstärkt worden. Da das Adressenmaterial der Gruppe der (zum Stichtag noch) Studierenden relativ aktuell ist, wurde entschieden, die Studierenden aus Kosten- und Zeitgründen postalisch zu befragen. Die Erhebung erfolgte (auch bei den Studierenden) nicht anonym.

Zur Frage des Stichprobenumfangs für Ereignisanalysen erwähnt Harrell (1997) Simulationsstudien, die gezeigt haben, dass der erwartete Fehler von geschätzten Überlebenswahrscheinlichkeiten für $p<m / 20$ kleiner als 0.05 und für $p<$ $m / 10$ kleiner als 0.1 war, wobei $m$ die Anzahl der Ereignisse und $p$ die Anzahl der zu schätzenden Parameter ist. Da beim vorliegenden Datensatz bei der Implementierung von (semi)parametrischen Ereignisdatenmodellen im Grundmodell $p \sim 15$ angenommen werden kann, erfordern die oben angegeben Fehler zwischen 150 und 300 Ereignisse (=Studienabschlüsse). Wählt man $m=$ $15 p=225$, so sind das knapp $20 \%$ aller Absolventen der beiden untersuchten Jahrgänge bis zum Stichtag. Gemäß dem Anteil an der Grundgesamtheit betrug demnach die erwünschte Stichprobengröße für System-Abbrecher etwa 270 und für Studierende 190.

Vor allem aufgrund von

- nicht mehr aktuellen Heimat- bzw. Elternadressen,

- nicht im Telefonbuch eingetragenen Telefonnummern (Wertkartenhandy, Geheimnummern), und

- nicht aktuellen Telefonnummern im amtlichen Telefonbuch

waren viele Studienabbrecher und Absolventen nicht oder nur sehr schwer erreichbar. Weniger häufige Gründe für die Nicht-Erreichbarkeit waren bei der Hauptstudie, dass

- die Eltern nicht bereit waren, die Telefonnummer ihres Sohnes oder Tochter weiterzugeben,

- die Eltern keinen Kontakt mehr zu ihrem Sohn oder ihrer Tochter hatten und ihnen die Telefonnummer nicht bekannt war,

- die betreffende Studiennabbrecherin oder Absolventin bereits verstorben ist. 
Daher wurden aus den beiden Schichten (Absolventen und System-Abbrecher) gemäß den Erfahrungen aus der Pilotphase doppelt so viele Individuen wie benötigt zufällig ausgewählt und versucht - innerhalb eines vorgegebenen Zeitrahmens $-\mathrm{zu}$ interviewen. Die erhaltene Stichprobe wurde dann in einigen wesentlichen Merkmalen mit der Grundgesamtheit verglichen, um eventuelle Verzerrungen (bezüglich dieser Merkmale) der Stichprobe gegenüber der Grundgesamtheit aufgrund von Nicht-Erreichbarkeit festzustellen und durch Nacherhebungen korrigieren zu können. Bezüglich der zusätzlich erhobenen Variablen ist natürlich kein Vergleich mit der Grundgesamtheit möglich.

Bei der postalischen Befragung der Studierenden wurde entsprechend den Erfahrungen nach der ersten Aussendung nur mit einer geringen Rücklaufquote von $25-30 \%$ gerechnet, und somit zur Erreichung der erwünschten Stichprobengröße etwa $70 \%$ der Immatrikulierten der beiden Jahrgänge zufällig ausgewählt und befragt.

Tatsächlich betrug der Anteil der verwertbaren (nicht anonym zurückgeschickten) Fragebögen $26 \%$. Die so erhaltene Stichprobe wurde mit der Grundgesamtheit in einigen Merkmalen verglichen. Aufgrund von überzufälligen Abweichungen in einigen Merkmalen wurde eine telefonische Nacherhebung durchgeführt, um die Abweichungen zu verringern. Abschließende Vergleiche zwischen der Grundgesamtheit und der Stichprobe werden in den folgenden Kapiteln beschrieben.

\section{Inhalt der Befragung}

Die Befragung betraf folgende Themen:

- Abschluss-Schulnoten aus Mathematik und Englisch

- Abschlussabsicht zu Studienbeginn

- zweite Ausbildung (zweites Studium außerhalb der Wirtschaftsuniversität, Fachhochschule, College, Lehrgang, etc.) neben dem WU-Studium; die Erhebung erfolgte je Semester und fragte nach Ausbildungen, die durchschnittlich mindestens fünf Wochenstunden im Semester Zeitaufwand bedeuteten. Zusätzlich wurde nach der vorwiegenden Art der zweiten Ausbildung und ihrem Bezug zum WU-Studium gefragt.

- (studienbezogene) Auslandssemester

- Berufsintensität während der Studienzeit an der Wirtschaftsuniversität (keine Ferientätigkeit); die Erhebung erfolgte je Semester im durchschnittlichen 
Tabelle 10.1: Vergleich der Definitionen der Studiendauer entsprechend den Definitionen (a), (b) und (c) für die befragten Studierenden

\begin{tabular}{|l|c|c|c|c|}
\hline Studierstatus & Anzahl & Studiendauer & Mittelwert & Std.abw. \\
\hline Studienabbrecher & \multirow{2}{*}{66} & entsprechend (a) & 7.1 & 5.2 \\
& & entsprechend (b) & 3.8 & 3.4 \\
& & entsprechend (c) & 3.6 & 3.5 \\
\hline Absolvent & 250 & entsprechend (a) & 12.9 & 2.7 \\
& & entsprechend (c) & 12.8 & 2.7 \\
\hline
\end{tabular}

Wochenstundenausmaß. Ergänzend wurde nach dem vorwiegenden Grund für die Erwerbstätigkeiten gefragt.

- Betreuung von Kindern während des WU-Studiums (je Semester)

Auch zwei offene Fragen nach

- selektiven Prüfungen im ersten und zweiten Studienabschnitt, und

- Maßnahmen, die nach Ansicht der Befragten die Universitätsleitung treffen müsste, um die Studiendauer zu verkürzen,

enthält der Fragebogen. Studienabbrecher wurden zusätzlich nach der vorwiegenden Tätigkeit nach dem Abbruch und ihrem Bezug zum abgebrochenen WU-Studium und zum Zeitpunkt der Befragung noch Studierende nach ihrer Abschlussabsicht befragt. Mehrfachnennungen waren bei den Fragen nach der vorwiegenden Art der zweiten Ausbildung, der Hauptursache für die Erwerbstätigkeit und der vorwiegenden Tätigkeit nach dem Studienabbruch nicht möglich.

Die Studiendaten betreffend wurde weiters nach dem Studienstatus (abgeschlossen, abgebrochen, im Gange) zum Befragungszeitpunkt und gegebenenfalls nach dem Abbruch- bzw. Abschlussdatum gefragt. Für die Befragungsdaten wurde dann auf Basis dieser Informationen eine weitere Definition (c) für die Studiendauer und für die Dauer bis zum Studienabbruch eingeführt.

(c) Die Studiendauer bzw. die Dauer bis zum Studienabbruch ist die Zeit zwischen Studienbeginn und dem im Fragebogen angegeben Datum des Studienabschlusses bzw. Studienabbruchs.

Wie erwartet zeigt Tabelle 10.1, dass bei den Absolventen zwischen der im Fragebogen angegebenen Studiendauer und der im System vermerkten Zeit 
zwischen Studienbeginn und Studienende kaum Unterschiede auftreten. Bei den Studienabbrechern überschätzt die Definition (a) die Studiendauer bis zum Studienabbruch wie erwartet (um etwa drei Semester) deutlich. Die entsprechend den Definitionen (b) und (c) ermittelten Werte unterscheiden sich nur minimal. Dies zeigt, dass die Definition (b) die eigentliche Dauer bis zum Studienabbruch von Immatrikulierten gut schätzt.

Der für jeden Studierenden der inländischen Immatrikulierten der Wintersemester 1990/91 und 1993/94 erzeugte Datensatz aus der Hörerevidenz, der

- für Absolventen die Studiendauer als Zeitraum zwischen dem Datum des Studienbeginns an der WU und dem des (ersten) Abschlusses eines WUStudiums (entsprechend Definition (a))

- für Studienabbrecher die Dauer bis zum Studienabbruch entsprechend den Definitionen (a) und (b)

- für Studierende die Studiendauer als Zeitraum zwischen dem Datum des Studienbeginns an der WU und dem Stichtag (rechts-zensierte Zeitdauer)

- die Studienstatus-Indikatorvariable (abgeschlossen, abgebrochen, noch im Gange), wobei für die beiden Studienabbruch-Definitionen (A) und (B) jeweils zwei Indikatorvariablen erzeugt wurden, sowie

- die aus den Stammdaten und Prüfungsdaten gebildeten Variablen

enthält, wurde mit den entsprechenden Daten aus der Befragung anhand der (abgefragten) Matrikelnummer zusammengeführt. Bei den semesterweise erhobenen zeitveränderlichen Variablen wie der Berufsintensität (in durchschnittlichen Wochenstunden), dem parallelen Betreiben einer zweiten Ausbildung und der Kinderbetreuung geht jedes Semester von Studienbeginn bis zum Ende des Beobachtungszeitraums als eigenes Merkmal ein. Der Fragebogen findet sich im Anhang B. 


\section{Kapitel 11}

\section{Deskriptive Analysen}

Wie im letzten Kapitel erwähnt, wird in der vorliegenden Arbeit einerseits auf die Systemdaten von (inländischen) Immatrikulationsjahrgängen der Wintersemester 1989/90 bis 1993/94 und andererseits auf die Befragungsdaten der beiden Immatrikulationjahrgänge der Wintersemester 1990/91 (im weiteren "Jahrgang 1990") und 1993/94 ("Jahrgang 1993") eingegangen. Diese beiden Studieneingangs-Kohorten werden in diesem Abschnitt hinsichtlich des Studienfortschritts (zum Stichtag) analysiert. Ziel dieser Analysen ist neben einer Beschreibung des Datensatzes ein erstes Aufzeigen von Zusammenhängen zwischen Studienerfolg (-dauer und -abbruch) und den verfügbaren potentiell erklärenden Variablen. Diese univariaten Analysen dienen den multivariaten Analysen der Kapitel 12 und 13 als Vorstudie.

Ergebnisse von Analysen von Absolventenjahrgängen (und StudienabbrecherJahrgängen) werden in dieser Arbeit nicht explizit präsentiert, können jedoch im Forschungsbericht Hackl und Sedlacek (2002) nachgelesen werden.

\subsection{Beschreibung des Studienfortschritts}

\section{Analyse der gesamten Population}

In Tabelle 11.1 wird der Studierstatus bzw. -fortschritt der Jahrgänge 1990 und 1993 zum Stichtag (31.12.00) beschrieben. Unter dem Begriff SystemAbbrecher werden Studienabbrecher gemäß Definition (A) aus Abschnitt 10.1 verstanden; System-Abbrecher sind somit Studierende, die vor dem Stichtag ihre an der Wirtschaftsuniversität begonnenen Studien abgebrochen haben. 
Tabelle 11.1: Studienfortschritt und Studierstatus der Jahrgänge 1990 und 1993 per 31.12 .2000

\begin{tabular}{|l|cc|cc|}
\hline & \multicolumn{2}{|c|}{ Jahrgang 1990 } & \multicolumn{2}{c|}{ Jahrgang 1993 } \\
\hline Inländische Immatrikulierte & 2015 & $100 \%$ & 1557 & $100 \%$ \\
\hline nach Studienfortschritt & & & & \\
mind. eine Leistung & 1672 & $83 \%$ & 1374 & $88 \%$ \\
mind. eine pos. Leistung & 1572 & $78 \%$ & 1277 & $82 \%$ \\
mind. eine pos. VP/DP & 1219 & $60 \%$ & 974 & $63 \%$ \\
im zweiten Abschnitt & 976 & $48 \%$ & 736 & $47 \%$ \\
\hline nach Studierstatus & & & & \\
Absolventen & 755 & $37 \%$ & 407 & $26 \%$ \\
System-Abbrecher & 873 & $43 \%$ & 535 & $34 \%$ \\
Studierende & 387 & $20 \%$ & 615 & $40 \%$ \\
\hline
\end{tabular}

Tabelle 11.2 beschreibt den Studierstatus der System-Abbrecher und der zum Stichtag noch (aktiven und nicht-aktiven) Studierenden (entsprechend den Definitionen aus Abschnitt 10.1) näher.

Tabelle 11.2: Studienfortschritt der System-Abbrecher und der Studierenden per 31.12 .2000

\begin{tabular}{|l|cc|cc|}
\hline & \multicolumn{2}{|c|}{ Jahrgang 1990 } & \multicolumn{2}{|c|}{ Jahrgang 1993 } \\
\hline System-Abbrecher & 873 & $100 \%$ & 535 & $100 \%$ \\
\hline nach Studienfortschritt & & & & \\
mind. eine Leistung & 538 & $62 \%$ & 363 & $68 \%$ \\
mind. eine pos. Leistung & 441 & $51 \%$ & 276 & $52 \%$ \\
mind. eine pos. VP/DP & 132 & $15 \%$ & 48 & $9 \%$ \\
im zweiten Abschnitt & 18 & $2 \%$ & 4 & $1 \%$ \\
\hline Studierende & 387 & $100 \%$ & 615 & $100 \%$ \\
\hline nach Studienfortschritt & & & & \\
mind. eine Leistung & 379 & $98 \%$ & 604 & $98 \%$ \\
mind. eine pos. Leistung & 376 & $97 \%$ & 594 & $97 \%$ \\
mind. eine pos. VP/DP & 332 & $86 \%$ & 519 & $84 \%$ \\
im zweiten Abschnitt & 203 & $52 \%$ & 325 & $53 \%$ \\
\hline
\end{tabular}

Die Tabellen 11.1 und 11.2 geben einen ersten groben Eindruck über Abschlussund Abbruchquoten sowie über die Studiendauer von inländischen WU-Imma- 
trikulierten. Etwa 20\% der Immatrikulierten der beiden Jahrgänge haben bis zum Stichtag keine einzige Lehrveranstaltung an der Wirtschaftsuniversität positiv absolviert. An diesen Zahlen wird sich kaum mehr etwas ändern, weil fast alle derzeit noch Studierenden bisher schon mindestens eine positive Leistung erbracht haben. Zum Stichtag macht diese Gruppe etwa $50 \%$ der SystemAbbrecher aus. Dieser Anteilswert wird zwar noch kleiner werden, da in den nächsten Jahren fast ausschließlich Studierende mit mindestens einer positiven Leistung abbrechen werden, jedoch liefern die vorliegenden Tabellen einen ersten Eindruck, wie die Studienabbrecher an der Wirtschaftsuniversität nach Studierstatus verteilt ist.

Je mehr Leistungen gebracht werden - etwa das positive Ablegen einer Vorbzw. Teildiplomprüfung oder die Absolvierung des ersten Studienabschnitts -, umso geringer ist die Wahrscheinlichkeit eines WU-Studienabbruchs. Analysen von Studienabbrechern eines bestimmten Zeitraums, etwa aller SystemAbbrecher des Studienjahres 1999/2000, können Aufschlüsse über die Verteilung der Studiendauer und des Studienfortschritts von Studienabbrechern liefern; siehe Hackl und Sedlacek (2002).

$\mathrm{Zu}$ beachten ist weiters, dass der Anteil der Immatrikulierten mit wenig Leistungen für den Jahrgang 1993 geringer als für den Jahrgang 1990 ist. Trendaussagen sind jedoch nur durch Vergleiche von mehreren Jahrgängen möglich.

Tabelle 11.3 beschreibt den Studienfortschritt der Studienabbrecher entsprechend der im Abschnitt 10.1 angeführten Definition (B) für den Studienabbruch, das sind System-Abbrecher und nicht-aktive Studierende.

Tabelle 11.3: Leistungen der System-Abbrecher und nicht-aktiven Studierenden

\begin{tabular}{|l|cc|cc|}
\hline & \multicolumn{2}{|c|}{ Jhg. 1990 } & \multicolumn{2}{c|}{ Jhg. 1993 } \\
\hline System-Abbrecher und n.a. Studierende & 1018 & $100 \%$ & 697 & $100 \%$ \\
\hline nach Studienfortschritt & & & & \\
mind. einer Leistung & 675 & $66 \%$ & 514 & $74 \%$ \\
mind. einer pos. Leistung & 575 & $56 \%$ & 417 & $60 \%$ \\
mind. einer pos. VP/DP & 236 & $23 \%$ & 132 & $19 \%$ \\
erstem Abschnitt & 50 & $5 \%$ & 9 & $1 \%$ \\
\hline
\end{tabular}

Mit der Definition (B) für den Studienabbruch erhöht sich die Zahl der Studienabbrecher zum Stichtag (gegenüber der Zahl der System-Abbrecher) für den Jahrgang 1990 von $43 \%$ auf $51 \%$ und für den Jahrgang 1993 von $34 \%$ auf $45 \%$ der Immatrikulierten. 
Tabelle 11.4 zeigt die Verteilung der positiven Lehrveranstaltungsprüfungen und Vor-bzw. Teildiplomprüfungen für die System-Abbrecher, die nicht-aktiven und aktiven Studierenden sowie für Absolventen der beiden Jahrgänge.

Tabelle 11.4: Leistungsprofil der Jahrgänge 1990 und 1993 nach Studierstatus

\begin{tabular}{|l|cc|cc|cc|cc|}
\hline & \multicolumn{2}{|c|}{ System-Abbr. } & \multicolumn{2}{|c|}{ n.a. Stud. } & \multicolumn{2}{c|}{ akt. Stud. } & \multicolumn{2}{c|}{ Absolv. } \\
& 1990 & 1993 & 1990 & 1993 & 1990 & 1993 & 1990 & 1993 \\
\hline pos. Leistungen & & & & & & & & \\
Mittelwert & 4.5 & 3.3 & 18.4 & 12.3 & 30.3 & 30.1 & 33.5 & 35 \\
Standardabw. & 6.9 & 5.2 & 10.4 & 8.7 & 8.1 & 8.4 & 5.3 & 5.4 \\
Median & 1 & 1 & 17 & 13 & 32 & 32 & 32 & 34 \\
\hline pos. VP/DP & & & & & & & & \\
Mittelwert & 0.4 & 0.2 & 2.8 & 1.3 & 7.9 & 7.8 & 11.8 & 11.8 \\
Standardabw. & 1.4 & 0.8 & 2.8 & 1.7 & 3.7 & 3.5 & 0.8 & 0.7 \\
Median & 0 & 0 & 2 & 2 & 9 & 9 & 12 & 12 \\
\hline
\end{tabular}

An Tabelle 11.4 ist zu erkennen, dass die nicht-aktiven Studierenden ein besseres durchschnittliches Leistungsprofil als die System-Abbrecher aufweisen bedingt durch die vielen System-Abbrecher ohne Leistungsnachweise - aber auch ein weitaus schlechteres als die aktiven Studierenden. Dies ist ein Hinweis dafür, dass die Definition (B) für den Studienabbruch geeignet ist. Das Leistungsprofil der aktiven Studierenden, die sich zu 70\% im zweiten Studienabschnitt befinden, im Vergleich mit dem der Absolventen deutet daraufhin, dass vermutlich die Mehrheit diese Kategorie noch ihr Studium abschließen wird.

\section{Analyse der Stichprobe}

Ergänzend wurde auch der Studienfortschritt der unter den inländischen Immatrikulierten der Wintersemester 1990 und 1993 gezogenen Stichprobe (siehe Abschnitt 10.2) untersucht. Die Ergebnisse geben Aufschluss darüber, inwiefern sich die Stichprobe von der Grundgesamtheit betreffend Studienfortschritt unterscheidet.

Tabelle 11.5 zeigt die Häufigkeitsverteilung der Stichprobe nach den am Stichtag gebildeten Schichten System-Abbrecher, Studierende und Absolventen und nach Jahrgang. Die Stichprobenanteile der drei Schichten können mit den Anteilswerten der Grundgesamtheit (siehe Tabelle 11.1) verglichen werden. Die Nullhypothese, dass die Stichprobenanteile gleich den entsprechenden Anteilen 
der Grundgesamtheit sind, kann unter Verwendung eines $\chi^{2}$-Anpassungstests nicht verworfen werden $(p=0.2)$.

Tabelle 11.5: Verteilung der Stichprobe nach Studierstatus und Jahrgang

\begin{tabular}{|l|cc|cc|cc|}
\hline & \multicolumn{2}{|c|}{ gesamt } & \multicolumn{2}{c|}{ Jhg. 1990 } & \multicolumn{2}{c|}{ Jhg. 1993} \\
Stichprobe & 703 & $100 \%$ & 389 & $100 \%$ & 314 & $100 \%$ \\
\hline Absolventen & 250 & $36 \%$ & 155 & $40 \%$ & 95 & $30 \%$ \\
System-Abbrecher & 260 & $37 \%$ & 160 & $41 \%$ & 100 & $32 \%$ \\
Studierende & 193 & $27 \%$ & 74 & $19 \%$ & 119 & $38 \%$ \\
\hline
\end{tabular}

Ergänzend zu den Auswertungen aus Tabelle 11.5 wurden für die Stichprobe Analysen zum Studienfortschritt entsprechend den Tabellen 11.1 und 11.2 durchgeführt und die Ergebnisse mit der Grundgesamtheit verglichen - mit dem Schluss, dass die betrachtete Stichprobe den Studienfortschritt betreffend keine überzufälligen Abweichungen von der Grundgesamtheit zeigt. Ein Vergleich der Verteilungen der Stichprobe und der gesamten Population bezüglich der demographischen Merkmale Geschlecht, Schultyp und Alter zeigte ebenfalls keine überzufälligen Unterschiede (siehe auch Abschnitt 11.2).

Zählt man zu den System-Abbrechern auch die nicht-aktiven Studierenden dazu, dann erhöht sich die Zahl der Studienabbrecher für den Jahrgang 1990 (um 21) auf 181 (47\%) und für den Jahrgang 1993 (um 16) auf 116 (37\%). Von diesen 37 nicht-aktiven Studierenden stufen sich selbst sechs bei der Befragung als Studienabbrecher ein (mit Angabe des Abbruchdatums). Drei Studierende sehen sich zwar als Studierende, geben aber an, das Studium nicht abschließen zu wollen, und weitere zehn sind sich nicht sicher, ob sie ihr WU-Studium noch abschließen wollen. Die restlichen 18 (49\%) nicht-aktiven Studierenden geben zwar an, dass sie das Studium noch abschließen wollen, aber ihr Leistungsprofil (durchschnittlich 1.5 positive Vor- bzw. Teildiplomprüfungen) lässt eher darauf schließen, dass sie potentielle Studienabbrecher sind. Von den restlichen 154 (aktiven) Studierenden stuft sich selbst niemand als Studienabbrecher ein. Drei (aktive) Studierende geben an, dass sie ihr Studium nicht abschließen möchten, und fünf $(3 \%)$ sind sich nicht sicher. Dies ist eine weitere Bestätigung dafür, dass die Definition (B) für den Studienabbruch passend ist und daher eine plausiblere Schätzung für die Abbruchquote zum Stichtag erlaubt.

Für die in der vorliegenden Arbeit durchgeführten Analysen der Stichprobe wurden zusätzlich zu den 260 System-Abbrechern auch jene sechs Studierenden, die sich im Fragebogen selbst als Abbrecher einstuften, zu den Studienabbrechern addiert. 
Tabelle 11.2 hat gezeigt, dass mit Stichtag etwa ein Drittel der System-Abbrecher (ca. $15 \%$ aller Immatrikulierten) keine einzige Leistung und knapp die Hälfte der System-Abbrecher (etwa 20\% aller Immatrikulierten) keine positive Leistung in ihrem WU-Studium erbracht haben. Bei diesen Personen ist zu hinterfragen, ob sie überhaupt als wirkliche WU-Studienanfänger definiert werden können. Daher wurde in der Befragung auch die Frage gestellt, ob zu Studienbeginn tatsächlich die Absicht bestanden hat, das Studium zu beenden oder nicht; vgl. Schneider und Weiländer (1990). Für die 266 Studienabbrecher in der Stichprobe zeigt Tabelle 11.6 die in der Befragung geäußerte Abschlussabsicht.

Tabelle 11.6: Abschlussabsicht der Studienabbrecher zu Studienbeginn

\begin{tabular}{|l|c|c|c|}
\hline \multirow{2}{*}{} & \multicolumn{3}{|c|}{ Abschlussabsicht zu Studienbeginn } \\
\cline { 2 - 4 } & ja & weiß nicht & nein \\
\hline Studienabbrecher (266) & $67.7 \%$ & $30.4 \%$ & $1.9 \%$ \\
\hline Studienabbrecher ohne Leistung (80) & $50.0 \%$ & $46.3 \%$ & $3.7 \%$ \\
\hline
\end{tabular}

Die Tabelle zeigt, dass der Anteil der Personen, die sich zu Studienbeginn nicht sicher waren, ob sie das begonnene WU-Studium auch abschließen wollen, unter den Studienabbrechern ohne Leistung deutlich höher ist als unter allen Studienabbrechern. Insgesamt war sich nur jeder zweite befragte Studienabbrecher ohne Leistung zu Studienbeginn sicher, das Studium auch abschließen $\mathrm{zu}$ wollen. Allerdings ist darauf hinzuweisen, dass die Ergebnisse aufgrund des retrospektiven Erhebungsdesigns verzerrt sein können.

Für die folgenden Kreuztabellen gilt, dass sich die Anteilswerte in der zweiten Zeile jeder Zelle auf die gesamte Stichprobe, die Werte in der dritten Zeile auf die Zeilensumme (Spalte "Total") und die Anteile in der vierten Zeile auf die Spaltensumme (Zeile "Total") beziehen.

Die Studienabbrecher wurden auch nach ihrer Hauptaktivität im ersten Jahr nach dem WU-Studium und deren Bezug zur Wirtschaftsuniversität gefragt. Tabelle 11.7 zeigt, dass knapp 60\% der Studienabbrecher angeben, dass sie im ersten Jahr nach ihrem Studienabbruch hauptsächlich einer Erwerbstätigkeit (Aktivität 3) nachgegangen sind - davon mehr als $50 \%$ mit WU-Bezug. Etwa ein Viertel der Studienabbrecher hat zu einem Studium an einer anderen Universität gewechselt (Aktivität 1) - großteils (71\%) zu einer Studienrichtung ohne Bezug zum WU-Studium. Aktivität 2 in der Tabelle bezeichnet eine andere Ausbildung als ein Universitätsstudium (z.B. Lehrgang, Fachhochschule). In der Aktivität 4 wurde meist Karenz, Mutterschaft oder ähnliches angegeben. 
Tabelle 11.7: Verteilung der Studienabbrecher nach Hauptaktivität (1: Universitätsstudium; 2: Lehrgang, FH; 3: Erwerbstätigkeit; 4: sonstige Aktivität) nach dem Studienabbruch und ihr Bezug zum WU-Studium

\begin{tabular}{|c|c|c|c|c|c|}
\hline WU-Bezug & & Akti & ität & & \\
\hline & 1 & 2 & 3 & 4 & Total \\
\hline mit Bezug & 20 & 13 & 88 & 1 & 122 \\
\hline & 7.55 & 4.91 & 33.21 & 0.38 & 46.04 \\
\hline & 16.39 & 10.66 & 72.13 & 0.82 & \\
\hline & 28.57 & 44.83 & 56.05 & 11.11 & \\
\hline ohne Bezug & 50 & 16 & 62 & 3 & 131 \\
\hline & 18.87 & 6.04 & 23.40 & 1.13 & 49.43 \\
\hline & 38.17 & 12.21 & 47.33 & 2.29 & \\
\hline & 71.43 & 55.17 & 39.49 & 33.33 & \\
\hline Bezug missing & 0 & 0 & 7 & 5 & 12 \\
\hline & 0.00 & 0.00 & 2.64 & 1.89 & 4.53 \\
\hline & 0.00 & 0.00 & 58.33 & 41.67 & \\
\hline & 0.00 & 0.00 & 4.46 & 55.56 & \\
\hline Total & 70 & 29 & 157 & 9 & 265 \\
\hline & 26.42 & 10.94 & 59.25 & 3.40 & 100.00 \\
\hline
\end{tabular}

Unterscheidet man zwischen Studienabbrechern, die nach eigenen Angaben in den ersten beiden Studiensemestern an der WU abgebrochen haben ("Frühabbrecher") oder später, so erhält man die Tabelle 11.8. Wie erwartet ist unter den "Frühabbrechern" der Anteil jener, die das Studium wechseln, fast doppelt so hoch (35\%) wie unter den Studienabbrechern, die nach dem zweiten Semester abgebrochen haben (18\%). An der Randverteilung der binären Variablen "Frühabbruch" (Spalte "Total") ist weiters zu erkennen, dass etwa die Hälfte der befragten Studienabbrecher angibt, in den ersten beiden Semester abgebrochen zu haben. Dieser Anteilswert wird sich noch etwas nach unten verändern, bestätigt aber die bisherigen Analysen, dass der Studienabbruch an der WU zu einem hohen Prozentsatz sehr früh im Studium erfolgt.

\subsection{Analyse des Studienfortschritts in Abhän- gigkeit von demographischen Variablen}

In diesem Abschnitt werden die beiden Immatrikulationsjahrgänge 1990 und 1993 anhand der im System verfügbaren Variablen Geschlecht, Alter (zu Stu- 
Tabelle 11.8: Verteilung der Studienabbrecher nach Hauptaktivität (1: Universitätsstudium; 2: Lehrgang, FH; 3: Erwerbstätigkeit; 4: sonstige Aktivität) im Jahr nach dem Studienabbruch und dem Zeitpunkt des Studienabbruchs

\begin{tabular}{|l|l|l|l|l|l|}
\hline & \multicolumn{4}{|c|}{ Aktiviät } & \\
\hline & 1 & 2 & 3 & 4 & Total \\
\hline Kein Frühabbruch & 24 & 14 & 92 & 5 & 135 \\
& 9.06 & 5.28 & 34.72 & 1.89 & 50.94 \\
& 17.78 & 10.37 & 68.15 & 3.70 & \\
& 34.29 & 48.28 & 58.60 & 55.56 & \\
\hline Frühabbruch & 46 & 15 & 65 & 4 & 130 \\
& 17.36 & 5.66 & 24.53 & 1.51 & 49.06 \\
& 35.38 & 11.54 & 50.00 & 3.08 & \\
\hline Total & 65.71 & 51.72 & 41.40 & 44.44 & \\
& 70 & 29 & 157 & 9 & 265 \\
& 26.42 & 10.94 & 59.25 & 3.40 & 100.00 \\
\hline
\end{tabular}

dienbeginn) und Schultyp näher beschrieben. Die Tabelle 11.9 beschreibt die Verteilung der inländischen Immatrikulierten der beiden betrachteten Jahrgänge und der Stichproben-Population nach diesen drei Variablen. Ein Vergleich der beiden Tabellen zeigt keine überzufälligen Unterschiede in den Verteilungen der betrachteten Variablen.

Um den Zusammenhang zwischen demographischen Variablen und dem Studienerfolg bzw. -fortschritt zu analysieren, werden in diesem Abschnitt Kreuztabellen der demographischen Variablen mit dem Studierstatus verwendet. Diese univariaten Analysen können erste Hinweise über die Bedeutung einzelner Variablen für detaillierte (multivariate) Analysen der Studiendauer und des Studienabbruch-Risikos geben.

In Tabelle 11.10 wird für den (inländischen) Immatrikulationsjahrgang des Wintersemesters 1993 der Zusammenhang zwischen Schultyp und Studierstatus (zum Stichtag) gezeigt.

Die Tabelle zeigt, dass es zwischen den Immatrikulierten je nach absolvierter Schulausbildung Unterschiede in der Verteilung nach dem Studierstatus gibt. Beim Vergleich von AHS- und HAK-Maturanten ist zum Stichtag eine annähernd gleiche Abbruchquote (ca. 30\%), jedoch eine um fast $10 \%$ höhere Abschlussquote von HAK-Absolventen abzulesen. Dies lässt einen ersten (groben) Schluss zu, dass HAK-Absolventen (bei etwa gleicher Abbruchquote) das Studium schneller abschließen. Ein Vergleich von HTL- und HAK-Absolventen 
Tabelle 11.9: Verteilungen der untersuchten Population und der StichprobenPopulation nach den Variablen Geschlecht, Alter und Vorbildung

\begin{tabular}{|l|c|c|c|}
\hline gesamte Population & gesamt & Jhg. 1990 & Jhg. 1993 \\
\hline Geschlecht & & & \\
weiblich & $47.4 \%$ & $47.4 \%$ & $47.5 \%$ \\
männlich & $52.6 \%$ & $52.6 \%$ & $52.5 \%$ \\
\hline Vorbildung & & & \\
AHS & $45.2 \%$ & $48.0 \%$ & $41.6 \%$ \\
HAK & $28.1 \%$ & $27.2 \%$ & $29.4 \%$ \\
HTL & $18.9 \%$ & $17.4 \%$ & $20.9 \%$ \\
HLA & $4.7 \%$ & $4.4 \%$ & $5.0 \%$ \\
Sonstige & $3.1 \%$ & $3.0 \%$ & $3.1 \%$ \\
\hline Alter (zu Studienbeginn) & & & \\
Mittelwert & 20.3 & 20.2 & 20.5 \\
Standardabweichung & 2.7 & 2.5 & 3.1 \\
Median & 19.6 & 19.5 & 19.6 \\
\hline \hline Stichproben-Population & gesamt & Jhg. 1990 & Jhg. 1993 \\
\hline Geschlecht & & & \\
weiblich & $47.0 \%$ & $47.0 \%$ & $46.8 \%$ \\
männlich & $53.0 \%$ & $53.0 \%$ & $53.2 \%$ \\
\hline Vorbildung & & & \\
AHS & $44.2 \%$ & $46.5 \%$ & $41.4 \%$ \\
HAK & $29.2 \%$ & $28.0 \%$ & $30.6 \%$ \\
HTL & $19.1 \%$ & $18.5 \%$ & $19.8 \%$ \\
HLA & $5.3 \%$ & $5.4 \%$ & $5.1 \%$ \\
Sonstige & $2.3 \%$ & $1.5 \%$ & $3.2 \%$ \\
\hline Alter (zu Studienbeginn) & & & \\
Mittelwert & 20.1 & 19.9 & 20.4 \\
Standardabweichung & 2.4 & 1.8 & 3.0 \\
Median & 19.6 & 19.5 & 19.7 \\
\hline
\end{tabular}

lässt darauf schließen, dass HTL-Absolventen nicht nur eine höhere Abbruchquote haben, sondern auch länger für den Studienabschluss brauchen. Geht man etwa von der Annahme aus, dass ein Großteil jener Studierenden, die zum Stichtag bereits im zweiten Abschnitt sind ("Student1"), das Studium noch abschließen wird, während jene, die bis zum Stichtag den ersten Abschnitt noch nicht absolviert haben ("Student0"), das Studium größtenteils abbrechen werden, dann kann man aus der Tabelle ablesen, dass von den 
Tabelle 11.10: Verteilung des Jahrgangs 1993 nach Vorbildung und Studierstatus (Student0: im ersten Abschnitt, Student1: im zweiten Abschnitt) per 31.12.2000

\begin{tabular}{|l|l|l|l|l|l|}
\hline \multirow{2}{*}{ Schultyp } & \multicolumn{4}{|c|}{ Studierstatus } & \multirow{2}{*}{ Total } \\
\cline { 2 - 5 } & System-Abbrecher & Student0 & Student1 & Absolvent & THS \\
\hline AH2 & 124 & 171 & 171 & 648 \\
& 11.69 & 7.96 & 10.98 & 10.98 & 41.62 \\
& 28.09 & 19.14 & 26.39 & 26.39 & \\
& 34.02 & 42.76 & 52.62 & 42.01 & \\
\hline HAK & 141 & 73 & 84 & 159 & 457 \\
& 9.06 & 4.69 & 5.39 & 10.21 & 29.35 \\
& 30.85 & 15.97 & 18.38 & 34.79 & \\
& 26.36 & 25.17 & 25.85 & 39.07 & \\
\hline HLA & 38 & 9 & 14 & 16 & 77 \\
& 2.44 & 0.58 & 0.90 & 1.03 & 4.95 \\
& 49.35 & 11.69 & 18.18 & 20.78 & \\
& 7.10 & 3.10 & 4.31 & 3.93 & \\
\hline HTL & 149 & 74 & 51 & 52 & 326 \\
& 9.57 & 4.75 & 3.28 & 3.34 & 20.94 \\
& 45.71 & 22.70 & 15.64 & 15.95 & \\
& 27.85 & 25.52 & 15.69 & 12.78 & \\
\hline Sonstige & 25 & 10 & 5 & 9 & 49 \\
& 1.61 & 0.64 & 0.32 & 0.58 & 3.15 \\
& 51.02 & 20.41 & 10.20 & 18.37 & \\
& 4.67 & 3.45 & 1.54 & 2.21 & \\
\hline Total & 535 & 290 & 325 & 407 & 1557 \\
& 34.36 & 18.63 & 20.87 & 26.14 & 100.00 \\
\hline
\end{tabular}

HAK-Maturanten bis zum Stichtag (nach ca. 14 Semester) etwa 2/3 der zu erwartenden Absolventen fertig geworden sind, während dieser Anteilswert bei den HTL-Absolventen (ähnlich wie bei den AHS-Absolventen) nur etwa bei $50 \%$ liegt. Analysen von Absolventenjahrgängen liefern ähnliche Aussagen betreffend Studiendauer; siehe Hackl und Sedlacek (2002).

(Deskriptive) Analysen der Studiendauer und des Studienabbruchs mit Hilfe von Kreuztabellen können nur erste Hinweise für potentielle Einflussgrößen geben. Folgende Punkte müssen bei der Interpretation der Kreuztabellen beachtet werden: 
- Einflussgrößen werden nur separat (univariat) und nicht simultan (multivariat) betrachtet, dadurch können Abhängigkeiten zwischen Variablen nicht berücksichtigt werden.

- Es wird nicht die (verfügbare) Information der exakten Studiendauer der Absolventen (und auch nicht der Dauer bis zum Abbruch) genützt, sondern nur unterschieden, in welchem Studierstatus sich der Studierende zum Stichtag befindet; die Zustände könnten natürlich noch beliebig verfeinert werden, die Interpretation wird dadurch jedoch komplexer.

- Aussagen über den Zusammenhang der Variablen mit der Studiendauer und der Studienabbruchquote setzen Annahmen über den weiteren Verlauf der Gruppe der zum Stichtag noch Studierenden voraus.

Neben den Zusammenhängen zwischen Schultyp und Studierstatus ist aus Tabelle 11.10 auch die Verteilung des Jahrgangs 1993 nach dem Matura-Schultyp (Randverteilung) in der Spalte "Total" und die Verteilung nach dem Studierstatus in der Zeile "Total" abzulesen. So haben knapp $42 \%$ der (inländischen) Immatrikulierten des Wintersemesters 1993 an einer AHS maturiert und knapp $30 \%$ an einer HAK; vgl. auch Tabelle 11.9. Zum Stichtag (nach etwa 14 Semester) zählen mehr als ein Viertel der Immatrikulierten zu den Absolventen und mehr als ein Drittel zu System-Abbrechern. Fast jeder fünfte Immatrikulierte befindet sich (offiziell) noch im ersten Abschnitt; vgl. auch Tabelle 11.1. Die für den Jahrgang 1993 getroffenen Aussagen konnten auch für die Jahrgänge 1990 bis 1992 bestätigt werden.

Neben den im System verfügbaren demographischen Variablen wie dem Geschlecht, dem Alter zu Studienbeginn und dem Schultyp wurden weitere Variablen erhoben, die nur für die Stichprobe verfügbar sind; siehe Abschnitt 10.2 .

Die Häufigkeitsverteilung der in der Stichprobe erhobenen Population nach den Schul-Abschlussnoten aus Mathematik und dem Studierstatus (zum Stichtag) ist in Tabelle 11.11 (über die beiden betrachteten Immatrikulationjahrgänge aggregiert) dargestellt. Die Tabelle lässt einen Zusammenhang zwischen der Schul-Abschlussnote aus Mathematik und dem Studienerfolg vermuten. So haben etwa zwei Drittel der Absolventen zum Stichtag in Mathematik mit den Noten Eins oder Zwei abgeschlossen, während dies bei den Studienabbrechern und den Studierenden nur etwa die Hälfte sind. Vier Befragte konnten sich nicht mehr an ihre Abschlussnoten aus Mathematik und Englisch erinnern.

Beinahe analoge Ergebnisse (und Interpretationen) erhält man für die SchulAbschlussnote aus Englisch (siehe Tabelle 11.12). Dies lässt den Schluss zu, 
Tabelle 11.11: Verteilung der Stichproben-Population nach der SchulAbschlussnote in Mathematik und dem Studierstatus per 31.12.2000

\begin{tabular}{|l|l|l|l|l|l|}
\hline \multirow{2}{*}{$\begin{array}{l}\text { Nathe- } \\
\text { Noten }\end{array}$} & \multicolumn{4}{|c|}{ Studierstatus } & \multirow{2}{*}{ Total } \\
\cline { 2 - 5 } 1 & Studienabbrecher & Student0 & Student1 & Absolvent & 149 \\
& 39 & 17 & 16 & 77 & 21.32 \\
& 26.58 & 2.43 & 2.29 & 11.02 & \\
& 14.83 & 11.41 & 10.74 & 51.68 & \\
\hline 2 & 86 & 20.48 & 15.53 & 30.80 & \\
& 12.30 & 25 & 29 & 90 & 230 \\
& 37.39 & 3.58 & 4.15 & 12.88 & 32.90 \\
& 32.70 & 10.87 & 12.61 & 10.87 & \\
\hline 3 & 105 & 30.12 & 28.16 & 36.00 & \\
& 15.02 & 27 & 43 & 60 & 235 \\
& 44.68 & 3.86 & 6.15 & 8.58 & 33.62 \\
& 39.92 & 11.49 & 18.30 & 25.53 & \\
\hline 4 & 33 & 32.53 & 41.75 & 24.00 & \\
& 4.72 & 14 & 15 & 23 & 85 \\
& 38.82 & 2.00 & 2.15 & 3.29 & 12.16 \\
& 12.55 & 16.47 & 17.65 & 27.06 & \\
\hline Total & 263 & 16.87 & 14.56 & 9.20 & \\
& 37.63 & 83 & 103 & 250 & 699 \\
& & 11.87 & 14.74 & 35.77 & 100.00 \\
\hline
\end{tabular}

dass es zwischen dem Studienerfolg und den Schulleistungen einen Zusammenhang gibt.

Die Variablen "zweite Ausbildung", "Auslandssemester", "Berufstätigkeit" und "Kinderbetreuung" wurden als zeitabhängige Variablen erhoben; siehe Abschnitt 10.2. Der dynamische Aspekt dieser Variablen wird in den Kapiteln 12 und 13 berücksichtigt. Bei der deskriptiven Analyse dieser Variablen ist zu beachten, dass die Werte dieser Variablen für den Stichtag gelten. Mit Fortschreiten der Zeit können sich sowohl der Studierstatus als auch die Werte der Variablen der (zum Zeitpunkt der Erhebung noch) Studierenden verändern.

Tabelle 11.13 zeigt, wieviele der Befragten (bis zum Befragungszeitpunkt) während ihrer WU-Studienzeit zumindest ein Semester lang (mit einem durchschnittlichen Zeitaufwand von mindestens fünf Wochenstunden) eine weitere Ausbildung neben ihrem WU-Studium besuchten.

Etwas weniger als ein Viertel der Befragten gibt an, parallel zum WU-Studium (bis zum Befragungszeitpunkt) eine weitere Ausbildung besucht zu haben. Da 
Tabelle 11.12: Verteilung der Stichproben-Population nach der SchulAbschlussnote in Englisch und dem Studierstatus per 31.12.2000

\begin{tabular}{|l|l|l|l|l|l|}
\hline \multirow{2}{*}{$\begin{array}{l}\text { Englisch- } \\
\text { Noten }\end{array}$} & \multicolumn{4}{|c|}{ Studierstatus } & \multirow{2}{*}{ Total } \\
\cline { 2 - 5 } & Studienabbrecher & Student0 & Student1 & Absolvent & 158 \\
& 50 & 15 & 19 & 74 & 158 \\
& 7.15 & 2.15 & 2.72 & 10.59 & 22.60 \\
& 31.65 & 9.49 & 12.03 & 46.84 & \\
\hline 2 & 19.01 & 18.07 & 18.45 & 29.60 & \\
\hline 3 & 89 & 20 & 33 & 92 & 234 \\
& 12.73 & 2.86 & 4.72 & 13.16 & 33.48 \\
& 38.03 & 8.55 & 14.10 & 39.32 & \\
& 33.84 & 24.10 & 32.04 & 36.80 & \\
\hline 3 & 94 & 28 & 33 & 66 & 221 \\
& 13.45 & 4.01 & 4.72 & 9.44 & 31.62 \\
& 42.53 & 12.67 & 14.93 & 29.86 & \\
& 35.74 & 33.73 & 32.04 & 26.40 & \\
\hline 4 & 30 & 20 & 18 & 18 & 86 \\
& 4.29 & 2.86 & 2.58 & 2.58 & 12.30 \\
& 34.88 & 23.26 & 20.93 & 20.93 & \\
\hline Total & 11.41 & 24.10 & 17.48 & 7.20 & \\
& 263 & 83 & 103 & 250 & 699 \\
& 37.63 & 11.87 & 14.74 & 35.77 & 100.00 \\
\hline
\end{tabular}

viele Studienabbrecher nur sehr kurz an der Wirtschaftsuniversität waren, ist es nicht überraschend, dass nur etwa $10 \%$ der Studienabbrecher während ihrer WU-Studienzeit zumindest ein Semester lang auch eine weitere Ausbildung besuchten. Interessanter ist der Unterschied zwischen Absolventen (18\%) und Studierenden des ersten und zweiten Studienabschnitts (51\% bzw. 37\%), der zeigt, dass "Langzeitstudierende" häufiger neben ihrem WU-Studium auch eine zweite Ausbildung besucht bzw. zumindest probiert haben. Bei der Interpretation muss allerdings beachtet werden, dass anhand dieser Tabelle allein nicht der kausale Schluss möglich ist, dass sich der Besuch einer zweiten Ausbildung verlängernd auf die Studiendauer auswirkt. Dazu ist die Berücksichtigung der Variablen "zweite Ausbildung" als zeitabhängige Variable notwendig, wie dies in einem Ereignisdatenmodell möglich ist; siehe Abschnitt 6.3.

Tabelle 11.14 gibt einen Überblick über die Art der neben dem WU-Studium parallel besuchten Ausbildung und die Einschätzung der Befragten, ob diese Ausbildung einen Bezug zu ihrem WU-Studium hat oder nicht. Die Bildungs- 
Tabelle 11.13: Verteilung der Stichproben-Population nach dem Besuch einer zweiten Ausbildung und dem Studierstatus per 31.12.2000

\begin{tabular}{|l|l|l|l|l|l|}
\hline \multirow{2}{*}{ Ausbildung } & \multicolumn{4}{|c|}{ Studierstatus } & \multirow{2}{*}{ Total } \\
\cline { 2 - 5 } & Studienabbrecher & Student0 & Student1 & Absolvent & 157 \\
\hline ja & 30 & 43 & 38 & 46 & 22.33 \\
& 4.27 & 6.12 & 5.41 & 6.54 & \\
& 19.11 & 27.39 & 24.20 & 29.30 & \\
\hline nein & 11.28 & 51.19 & 36.89 & 18.40 & \\
& 236 & 41 & 65 & 204 & 546 \\
& 33.57 & 5.83 & 9.25 & 29.02 & 77.67 \\
& 43.22 & 7.51 & 11.90 & 37.36 & \\
\hline Total & 88.72 & 48.81 & 63.11 & 81.60 & \\
& 266 & 84 & 103 & 250 & 703 \\
& 37.84 & 11.95 & 14.65 & 35.56 & 100.00 \\
\hline
\end{tabular}

angebote "Fachhochschule" und "College" wurden in Tabelle 11.14 wegen geringer Fallzahlen mit der Kategorie "Sonstige" zusammengefasst. Somit wird bei der Bildungsart nur zwischen einem zweiten Universitätsstudium (außerhalb der Wirtschaftsuniversität) und "Sonstige" unterschieden.

Tabelle 11.14: Verteilung der Studierenden mit paralleler Ausbildung nach der Art der zweiten Ausbildung und dem Bezug zum WU-Studium per 31.12.2000

\begin{tabular}{|l|l|l|l|l|}
\hline \multirow{2}{*}{ Bildungsart } & \multicolumn{3}{|c|}{ Bezug zum WU-Studium } & \multirow{2}{*}{ Total } \\
\cline { 2 - 4 } & mit Bezug & ohne Bezug & Bezug missing & 89 \\
\hline Univ.- & 22 & 61 & 6 & 57.05 \\
Studium & 14.10 & 39.10 & 3.85 & \\
& 24.72 & 68.54 & 6.74 & \\
\hline Sonstige & 36.67 & 73.49 & 46.15 & 67 \\
& 38 & 22 & 7 & 42.95 \\
& 24.36 & 14.10 & 4.49 & \\
& 56.72 & 32.84 & 10.45 & 156 \\
\hline Total & 63.33 & 26.51 & 53.85 & 100.00 \\
\hline
\end{tabular}

Bis zum Befragungszeitpunkt besuchten (während ihrer WU-Studienzeit) mehr als die Hälfte der Befragten mit einem weiteren Bildungsangebot zumindest 
Tabelle 11.15: Häufigkeitsverteilung der Studierenden mit paralleler Ausbildung nach der Anzahl der für diese Ausbildung beanspruchten Semester

\begin{tabular}{|l|l|l|l|l|}
\hline Semester & Anzahl & Anteil & $\begin{array}{l}\text { kumulierte } \\
\text { Anzahl }\end{array}$ & $\begin{array}{l}\text { kumulierte } \\
\text { Anteile }\end{array}$ \\
\hline 1 & 43 & 27.39 & 43 & 27.39 \\
2 & 33 & 21.02 & 76 & 48.41 \\
3 & 19 & 12.10 & 95 & 60.51 \\
4 & 23 & 14.65 & 118 & 75.16 \\
$>=5$ & 39 & 24.84 & 157 & 100.00 \\
\hline
\end{tabular}

ein Semester lang noch ein weiteres Universitätsstudium (zumeist ohne Bezug zu ihrem WU-Studium). Sonstige Bildungsformen - häufig sind hier Lehrgänge an der Wirtschaftsuniversität angegeben worden - wurden zu mehr als $50 \%$ mit Bezug zum WU-Studium gewählt. 13 Studierende (8\%) gaben nicht an, ob das besuchte Bildungsangebot mit Bezug zum WU-Studium war oder nicht, und ein weiterer gab die Bildungsart nicht an.

Tabelle 11.15 gibt einen Überblick, wie intensiv die weiteren Ausbildungen betrieben worden sind. Etwa ein Viertel der Studierenden mit zweitem Bildungsangebot, das sind etwa $6 \%$ aller Befragten, hat diese Ausbildung (bis zum Befragungszeitpunkt) nur ein Semester parallel zum WU-Studium besucht. Ein ähnlicher Anteil von Studierenden hat mehr als vier Semester in eine weitere Ausbildung parallel zum WU-Studium investiert.

Zusammenfassend lassen die Tabellen 11.13 und 11.15 den Schluss zu, dass die Studierenden der beiden Immatrikulationsjahrgänge eher wenig Zeit für eine zweite Ausbildung neben dem WU-Studium investiert haben. Dieses Ergebnis ist vergleichbar mit den Auswertungen innerhalb der ÖH-Studie (Grossmann et al. (1999b)), in der $83 \%$ der befragten Studierenden wirtschaftswissenschaftlicher Studienrichtungen angeben, in den letzten beiden Semestern nicht ernsthaft eine weitere Studienrichtung betrieben zu haben.

Tabelle 11.16 zeigt, wieviele Befragte bis zum Befragungszeitpunkt im Zuge ihres WU-Studiums zumindest ein Semester lang im Ausland waren (Auslandssemster). An der Tabelle ist vor allem hervorzuheben, dass etwa zwei Drittel der Befragten, die ein Auslandssemester angeben, bereits Absolventen sind. Unter den Absolventen (zum Stichtag) selbst hat mehr als ein Drittel (zumindest) ein Semester im Ausland verbracht. Diese Ergebnisse entsprechen den Ergebnissen einer aktuellen Studie des Vizerektorats für internationale Beziehungen, in der zwar $50 \%$ der Absolventen einen Auslandsaufenthalt im 
Rahmen des Studiums angeben, aber nur 35\% zumindest ein ganzes Semester im Ausland verbracht haben; siehe Mautner und Brandenburg (2001).

Tabelle 11.16: Verteilung der Stichproben-Population nach dem Absolvieren eines Auslandssemesters und dem Studierstatus per 31.12.2000

\begin{tabular}{|l|l|l|l|l|l|}
\hline Auslands- & \multicolumn{4}{|c|}{ Studierstatus } & \\
\cline { 2 - 5 } semester & Studienabbrecher & Student0 & Student1 & Absolvent & Total \\
\hline ja & 10 & 9 & 21 & 89 & 129 \\
& 1.42 & 1.28 & 2.99 & 12.66 & 18.35 \\
& 7.75 & 6.98 & 16.28 & 68.99 & \\
& 3.76 & 10.71 & 20.39 & 35.60 & \\
\hline nein & 256 & 75 & 82 & 161 & 574 \\
& 36.42 & 10.67 & 11.66 & 22.90 & 81.65 \\
& 44.60 & 13.07 & 14.29 & 28.05 & \\
& 96.24 & 89.29 & 79.61 & 64.40 & \\
\hline Total & 266 & 84 & 103 & 250 & 703 \\
& 37.84 & 11.95 & 14.65 & 35.56 & 100.00 \\
\hline
\end{tabular}

Da die Universitätsleitung großes Interesse an aktuellen Daten zur Berufstätigkeit der WU-Studierenden hat, wird diese Variable etwas ausführlicher beschrieben. Tabelle 11.17 zeigt, wieviele Befragte während ihres WU-Studiums bis zum Befragungszeitpunkt zumindest ein Semester lang einer Berufstätigkeit mit einem durchschnittlichen Zeitaufwand von mindestens fünf Wochenstunden nachgegangen sind.

Mehr als zwei Drittel der Befragten geben demnach an, während ihrer WUStudienzeit zumindest ein Semester lang einer Berufstätigkeit mit einem durchschnittlichen Zeitaufwand von mindestens fünf Wochenstunden nachgegangen zu sein, besonders hoch ist der Anteil dieser Gruppe unter den aktuell Studierenden (ca. 95\%). An diesen Anteilswerten wird sich kaum mehr etwas ändern, da schon jetzt - wie erwähnt - fast alle (zum Stichtag der Erhebung noch) Studierenden während ihres Studiums zumindest ein Semester berufstätig waren. Diese Zahlen stärken zwar die Vermutung, dass Erwerbstätigkeit neben dem Studium die Studiendauer verlängert. Eine genauere Analyse des Effekts der Berufstätigkeit auf die Studiendauer muss die Variable Berufstätigkeit zeitabhängig betrachten und die Berufsintensität berücksichtigen.

In Booth und Satchell (1995) und Yamaguchi (1991) (siehe Abschnitt 9.3) sind für die Analyse der Studiendauer und des -abbruchs Ereignisdatenmodelle implementiert worden. In beiden Studien wurde abgefragt, ob der Studieneintritt 
Tabelle 11.17: Verteilung der Stichproben-Population nach dem Nachgehen einer Erwerbstätigkeit und dem Studierstatus per 31.12.2000

\begin{tabular}{|l|l|l|l|l|l|}
\hline \multirow{2}{*}{$\begin{array}{l}\text { Erwerbs- } \\
\text { tätigkeit }\end{array}$} & \multicolumn{4}{|c|}{ Studierstatus } & \multirow{2}{*}{ Total } \\
\cline { 2 - 5 } ja & Studienabbrecher & Student0 & Student1 & Absolvent & Ta \\
& 160 & 80 & 98 & 158 & 496 \\
& 22.76 & 11.38 & 13.94 & 22.48 & 70.55 \\
& 32.26 & 16.13 & 19.76 & 31.85 & \\
& 60.15 & 95.24 & 95.15 & 63.20 & \\
\hline nein & 106 & 4 & 5 & 92 & 207 \\
& 15.08 & 0.57 & 0.71 & 13.09 & 29.45 \\
& 51.21 & 1.93 & 2.42 & 44.44 & \\
& 39.85 & 4.76 & 4.85 & 36.80 & \\
\hline Total & 266 & 84 & 103 & 250 & 703 \\
& 37.84 & 11.95 & 14.65 & 35.56 & 100.00 \\
\hline
\end{tabular}

als Teilzeit- oder Vollzeitstudent erfolgt ist, und diese Variable als zeitkonstantes Merkmal in das Modell aufgenommen. Auch in der ÖH-Studie (Grossmann et al. (1999a)) ist dieses Merkmal erhoben worden; siehe Abschnitt 9.1. In der vorliegenden Arbeit wurde zwar nicht nach dem Status Teilzeit- oder Vollzeitstudent zu Studienbeginn gefragt; allerdings können die Befragten nach ihrem Erwerbstätigkeitsstatus im ersten Semester unterschieden werden.

In Tabelle 11.18 wird die Erwerbstätigkeit in die folgenden vier Kategorien unterteilt:

1. nicht berufstätig bei weniger als 5 Wochenstunden

2. geringfügig beschäftigt bei mindestens 5 und weniger als durchschnittlich 15 Wochenstunden

3. teilzeit beschäftigt bei mindestens 15 und weniger als 30 Wochenstunden

4. vollzeit beschäftigt bei mindestens 30 Wochenstunden

Ein knappes Drittel (31\%) der Befragten ist im ersten Semester zumindest 15 Wochenstunden berufstätig gewesen (teilzeit oder vollzeit beschäftigt), unter den Studienabbrechern (zum Stichtag) und den Studierenden im ersten Abschnitt ist dieser Anteil jedoch mit $43 \%$ bzw. 38\% deutlich höher als bei den Absolventen und Studierenden im zweiten Abschnitt mit 22\% bzw. 20\%. Zwei Drittel der im ersten Semester Vollbeschäftigten zählen zum Stichtag zu den 
Tabelle 11.18: Verteilung der Stichproben-Population nach dem Erwerbstätigkeitsstatus im ersten Semester und dem Studierstatus per 31.12.2000

\begin{tabular}{|l|l|l|l|l|l|}
\hline \multirow{2}{*}{$\begin{array}{l}\text { Erwerbs- } \\
\text { status }\end{array}$} & \multicolumn{4}{|c|}{ Studierstatus } & \multirow{2}{*}{ Total } \\
\cline { 2 - 5 } geringfügig & Studienabbrecher & Student0 & Student1 & Absolvent & 66 \\
beschäftigt & 2.99 & 4 & 8 & 33 & 6.14 \\
& 31.82 & 0.57 & 1.14 & 4.70 & 9.40 \\
& 7.89 & 6.06 & 12.12 & 50.00 & \\
\hline nicht & 132 & 4.76 & 7.84 & 13.20 & \\
beschäftigt & 18.80 & 48 & 74 & 163 & 417 \\
& 31.65 & 6.84 & 10.54 & 23.22 & 59.40 \\
& 49.62 & 11.51 & 17.75 & 39.09 & \\
\hline teilzeit & 45 & 57.14 & 72.55 & 65.20 & \\
beschäftigt & 6.41 & 17 & 6 & 45 & 113 \\
& 39.82 & 2.42 & 0.85 & 6.41 & 16.10 \\
& 16.92 & 15.04 & 5.31 & 39.82 & \\
\hline vollzeit & 68 & 20.24 & 5.88 & 18.00 & \\
beschäftigt & 9.69 & 15 & 14 & 9 & 106 \\
& 64.15 & 2.14 & 1.99 & 1.28 & 15.10 \\
& 25.56 & 14.15 & 13.21 & 8.49 & \\
\hline Total & 266 & 17.86 & 13.73 & 3.60 & \\
& 37.89 & 84 & 102 & 250 & 702 \\
& & 11.97 & 14.53 & 35.61 & 100.00 \\
\hline
\end{tabular}

Tabelle 11.19: Durchschnittliche Berufsintensität (in Wochenstunden) während der WU-Studienzeit nach Studierstatus

\begin{tabular}{|l|l|l|l|l|}
\hline Studierstatus & Anzahl & Mittelwert & Std.abw. & Median \\
\hline Studienabbrecher & 266 & 16.08 & 15.94 & 12.92 \\
Student0 & 84 & 23.5 & 12.0 & 24.0 \\
Student1 & 103 & 15.7 & 10.9 & 15.0 \\
Absolvent & 250 & 9.17 & 9.93 & 7.56 \\
\hline
\end{tabular}

Studienabbrechern. Aus dieser Tabelle lässt sich somit ein erster grober Schluss ziehen, dass der Erwerbstätigkeitsstatus im ersten Semester vor allem auf die Studienabbruch-Wahrscheinlichkeit einen Einfluss hat.

Das Berufsausmaß während der WU-Studienzeit - getrennt nach Studierstatus zum Stichtag - beschreibt Tabelle 11.19. Die Tabelle macht deutlich, dass die Studierenden und die Studienabbrecher (zum Stichtag) während ihrer Studi- 
enzeit durchschnittlich deutlich mehr Zeit in eine Erwerbstätigkeit investierten als die Absolventen - unter den Studierenden gibt es auch deutliche Unterschiede je nach Studienfortschritt. Das durchschnittliche Berufsausmaß während der WU-Studienzeit selbst ist mit etwa 15 Wochenstunden ziemlich hoch.

Tabelle 11.20 zeigt die Verteilung der Antworten auf die Frage nach dem vorwiegenden Grund für die Berufstätigkeit, getrennt nach Studierstatus. Von den (berufstätigen) Studienabbrechern und den Studierenden des ersten Studienabschnitts geben $61 \%$ bzw. $66 \%$ Existenzsicherung als Hauptgrund an, von den Studierenden im zweiten Abschnitt ist ebenfalls Existenzsicherung der häufigste Grund (47\%), jedoch weniger häufig genannt als bei den Studierenden des ersten Abschnitts; die Absolventen hingegen führen das Zusatzeinkommen und auch die Berufschancen noch vor der Existenzsicherung an.

Tabelle 11.20: Verteilung der Berufstätigen nach dem (vorwiegenden) Grund für die Erwerbstätigkeit und dem Studierstatus per 31.12.2000

\begin{tabular}{|l|l|l|l|l|l|}
\hline \multirow{2}{*}{$\begin{array}{l}\text { Erwerbstätig- } \\
\text { keits-Grund }\end{array}$} & Studienabbrecher & Student0 & Student1 & Absolvent & \multirow{2}{*}{ Total } \\
\hline Existenz- & 95 & 53 & 46 & 34 & 228 \\
sicherung & 19.35 & 10.79 & 9.37 & 6.92 & 46.44 \\
& 41.67 & 23.25 & 20.18 & 14.91 & \\
& 60.90 & 66.25 & 46.94 & 21.66 & \\
\hline Zusatz- & 42 & 10 & 27 & 66 & 145 \\
einkommen & 8.55 & 2.04 & 5.50 & 13.44 & 29.53 \\
& 28.97 & 6.90 & 18.62 & 45.52 & \\
& 26.92 & 12.50 & 27.55 & 42.04 & \\
\hline Berufschancen & 9 & 16 & 18 & 47 & 90 \\
& 1.83 & 3.26 & 3.67 & 9.57 & 18.33 \\
& 10.00 & 17.78 & 20.00 & 52.22 & \\
& 5.77 & 20.00 & 18.37 & 29.94 & \\
\hline Sonstige & 10 & 1 & 7 & 10 & 28 \\
& 2.04 & 0.20 & 1.43 & 2.04 & 5.70 \\
& 35.71 & 3.57 & 25.00 & 35.71 & \\
& 6.41 & 1.25 & 7.14 & 6.37 & \\
\hline Total & 156 & 80 & 98 & 157 & 491 \\
& 31.77 & 16.29 & 19.96 & 31.98 & 100.00 \\
\hline
\end{tabular}

Weiters wurde noch erhoben, ob und wann die Befragten während ihrer WUStudienzeit mit Kindern im gemeinsamen Haushalt lebten. Die Fragestellung 
begründet sich in der Vermutung, dass die Betreuung von Kindern viel Zeitaufwand bedeutet und eine Verzögerung des Studiums zur Folge hat bzw. das Risiko des Studienabbruchs erhöht. Tabelle 11.21 zeigt, dass (zum Stichtag) nur wenig Befragte angeben, während ihrer WU-Studienzeit Kinder betreut zu haben. Zwar ist der Anteil von den (zum Stichtag noch) Studierenden mit Kinderbetreuung erwartungsgemäß deutlich höher (ca. $14 \%$ bzw. $10 \%$ je nach Studienfortschritt) als bei den Absolventen, doch die geringen Fallzahlen relativieren die Bedeutung dieser Variablen.

Tabelle 11.21: Verteilung der Stichproben-Population nach der Betreuung von Kindern und dem Studierstatus per 31.12.2000

\begin{tabular}{|l|l|l|l|l|l|}
\hline \multirow{2}{*}{$\begin{array}{l}\text { Kinder- } \\
\text { betreuung }\end{array}$} & \multicolumn{4}{|c|}{ Studierstatus } & \\
\cline { 2 - 5 } ja & Studienabbrecher & Student0 & Student1 & Absolvent & Total \\
\hline & 1.14 & 12 & 10 & 7 & 37 \\
& 21.62 & 1.71 & 1.42 & 1.00 & 5.26 \\
& 3.01 & 32.43 & 27.03 & 18.92 & \\
\hline nein & 258 & 14.29 & 9.71 & 2.80 & \\
& 36.70 & 72 & 93 & 243 & 666 \\
& 38.74 & 10.24 & 13.23 & 34.57 & 94.74 \\
& 96.99 & 10.81 & 13.96 & 36.49 & \\
\hline Total & 266 & 85.71 & 90.29 & 97.20 & \\
& 37.84 & 84 & 103 & 250 & 703 \\
& & 11.95 & 14.65 & 35.56 & 100.00 \\
\hline
\end{tabular}

In den beiden folgenden Kapiteln 12 und 13 wird mit Hilfe der im ersten Teil dieser Arbeit präsentierten Methoden der Ereignisanalyse der Einfluss von demographischen Variablen auf die Studiendauer und das StudienabbruchRisiko detailliert behandelt. 


\section{Kapitel 12}

\section{Analyse der Studiendauer und der Studienabschlussrate mit Hilfe von Ereignisdatenmodellen}

Im Mittelpunkt der beiden folgenden Kapiteln steht die Ermittlung von Einflussgrößen auf die Studiendauer, den Studienerfolg und das StudienabbruchRisiko und ihre Interpretation. Als Datenbasis stehen die in Kapitel 10 definierten System- und Befragungsdaten zur Verfügung.

\subsection{Einleitung}

In diesem Kapitel werden log-lineare parametrische Ereignisdatenmodelle (vgl. Kapitel 5) auf die vorhandenen Daten der Hörerevidenz (vgl. Abschnitt 10.1) angewandt, um die (Verteilung der) Studiendauer für verschiedene Gruppen von Studierenden zu schätzen.

In einem ersten Schritt werden die zu Studienbeginn für jeden Studierenden zur Verfügung stehenden Stammdaten herangezogen. Es wird untersucht, ob und in welchem Ausmaß diese Variablen Einfluss auf die Studiendauer haben. Danach werden die im System verfügbaren Prüfungsdaten des ersten Studienjahres hinzugezogen, um zu analysieren, inwiefern die Einbeziehung dieser Daten verlässliche Schätzungen der Studiendauer erlauben. Da diese Variablen im System verfügbar sind, lassen sich derartige Analysen routinemäßig durchführen.

Im Mittelpunkt der Analysen mit Hilfe von log-linearen parametrischen Modellen steht somit das Ziel, die Studiendauer für verschiedene Teilpopulationen 
von Immatrikulationsjahrgängen zu schätzen und den Einfluss bestimmter Variablen zu ermitteln.

Während parametrische Modelle eine Verteilungsannahme für die Studiendauer voraussetzen und die Ergebnisse von der angenommenen Verteilung abhängen, benötigen semiparametrische Modelle diese Annahme nicht. Bei der Analyse mit semiparametrischen Cox-Modellen interessiert nicht die Verteilung der Studiendauer, sondern es wird der Frage nachgegangen, welche Variablen besonderen Einfluss auf die Hazardrate für den Studienabschluss ausüben. So weit möglich werden die Ergebnisse der beiden Modellansätze verglichen. Zusätzlich steht insbesondere die Implementierung von zeitabhängigen Variablen im Mittelpunkt.

Unabhängig von der Modellierung - parametrisch oder semiparametrisch wird in diesem (und im folgenden) Kapitel der im Abschnitt 7.1 behandelte competing risks-Ansatz für kontinuierliche Modelle implementiert. Dabei werden die beiden Ereignisarten Studienabschluss $(m=1)$ und Studienabbruch $(m=2)$ unterschieden und jene Studierenden, die bis zum Stichtag weder abgebrochen noch abgeschlossen haben, gehen als Typ I rechts-zensierte Fälle in die Schätzung ein. Die beiden Ereignisarten können nicht als unabhängig vorausgesetzt werden, jedoch ist anzunehmen, dass ein Großteil der Abhängigkeit durch das Einbeziehen von geeigneten Kovariablen erklärt werden kann.

\subsection{Analyse der Studiendauer - log-lineare parametrische Modelle}

$\mathrm{Zu}$ Beginn dieses Abschnitts wird anhand des inländischen Immatrikulationjahrgangs des Wintersemesters 1990/91 (=Jahrgang 1990) detailliert auf die Modellentwicklung eingegangen. Ergebnisse werden für diesen Jahrgang und ergänzend auch für weitere Immatrikulationsjahrgänge gezeigt.

\section{Vorstudie: Parametrische Modelle ohne Kovariablen}

In diesem Unterabschnitt wird die Gestalt der durchschnittlichen (übergangsspezifischen) Hazardrate für den Studienabschluss, d.h. ohne Einbeziehung von Kovariablen, untersucht. Das Modell, das die beste Anpassung an die Daten liefert, wird dann als Basis für die darauffolgenden Analysen verwendet.

Bei der Modellierung der Dauer bis zum Studienabschluss ist zu beachten, dass die Mindeststudiendauer an der Wirtschaftsuniversität acht Semester beträgt. 
Daher werden die ersten acht Semester als Periode definiert, in der die Hazardrate für den Studienabschluss gleich Null ist. In der Schätzung der Modelle für die Studiendauer werden dann nur jene Studierenden berücksichtigt, die länger als acht Semester an der Wirtschaftsuniversität studieren; dadurch geht für den Jahrgang 1990 die Information von neun Studienabschlüssen (ca. 1\% der bis zum Stichtag erfolgten Abschlüsse) verloren, die bereits vor Ende des achten Semesters erfolgt sind. Diese Periode von acht Semestern wird als Garantiezeit (engl. guarantee time) bezeichnet und wird bei der Schätzung der Verteilung der Studiendauer berücksichtigt, indem der Beginn der Beobachtungszeit auf den Beginn des neunten Semesters gesetzt wird. Die Modellierung erfolgt somit in Abhängigkeit der (in Monaten modellierten) Reststudiendauer $t_{1}=t-48$. Es sei noch erwähnt, dass bei alternativer Implementierung einer Garantiezeit von sieben Semestern bei den folgenden Analysen praktisch keine Unterschiede in den Ergebnissen aufgetreten sind. Für die folgenden Analysen wurde die Studiendauer bis zum Abschluss analog der Definition (a) und die Zeitdauer bis zum Studienabbruch analog Definition (b) aus Abschnitt 10.1 verwendet.

Aufgrund von Vorstudien und Erfahrungswerten ist anzunehmen, dass die durchschnittliche (übergangsspezifische) Hazardrate für den Studienabschluss ab dem neunten Semester einige Semester steigt und dann leicht zu fallen beginnt. Diese Form der Hazardrate kann etwa mit einem log-logistischen- oder einem Gamma-Modell erreicht werden.

Diese Annahme kann über einen Vergleich der logarithmierten Werte der Likelihoodfunktion für alternative Verteilungsannahmen überprüft werden; Tabelle 12.1 zeigt die Log-Likelihood- und $A I C$-Werte ohne Einbeziehung von Kovariablen.

Tabelle 12.1: Log-Likelihood- und $A I C$-Werte für verschiedene Verteilungsannahmen für die Reststudiendauer

\begin{tabular}{|l|c|c|}
\hline Modell & Log-Likelihood & AIC-Wert \\
\hline Exponential & -1407.68 & 2817.36 \\
Weibull & -1328.25 & 2660.50 \\
Log-normal & -1333.36 & 2670.72 \\
Gamma & -1318.75 & 2643.50 \\
LLogistic & -1312.07 & 2628.14 \\
\hline
\end{tabular}

Das Exponential-, Weibull- und log-normale Modell sind Spezialfälle des verallgemeinerten Gamma-Modells. Alle Tests (mit Hilfe der Likelihood-QuotientenStatistik), dass die Nullhypothesen der entsprechenden Restriktionen zutreffen, 
führen mit $p$-Werten $<0.002$ zur Ablehnung. Unter den geschachtelten Modellen ist somit das Gamma-Modell das beste. Für das log-logistische Modell kann kein formaler Vergleich mit den anderen Modellen durchgeführt werden, allerdings liefert das log logistische Modell den kleinsten $A I C$-Wert. Das $A I C$-Kriterium einerseits und die einfachere Gestalt (und Interpretierbarkeit) sowie geringere Rechenintensität des log-logistischen Modells gegenüber dem Gamma-Modell andererseits führen zu dem Schluss, dass das log-logistische das geeignetste Modell zur Beschreibung der Daten ist.

Abbildung 12.1 zeigt die in Abschnitt 5.3 beschriebene graphische Überprüfung für die log-logistische Verteilungsannahme. Der Plot von $\ln \left\{\left[1-\hat{S}_{1}\left(t_{1}\right)\right] /\left[\hat{S}_{1}\left(t_{1}\right)\right]\right\}$ gegen $\ln \left(t_{1}\right)$ ist annähernd linear, wobei $\hat{S}_{1}\left(t_{1}\right)$ der Kaplan-Meier-Schätzer der in Abschnitt 7.1 definierten übergangsspezifischen Überlebensfunktion und $t_{1}$ die (über der Mindeststudiendauer liegende) Reststudiendauer an der WU ist. Ergänzend wurde für die Beziehung zwischen $\ln \left\{\left[1-\hat{S}_{1}\left(t_{1}\right)\right] /\left[\hat{S}_{1}\left(t_{1}\right)\right]\right\}$ und $\ln \left(t_{1}\right)$ auch ein lineares Regressionsmodell geschätzt $\left(R^{2}=0.99\right)$ und die Regressionsgerade in die Graphik eingetragen.

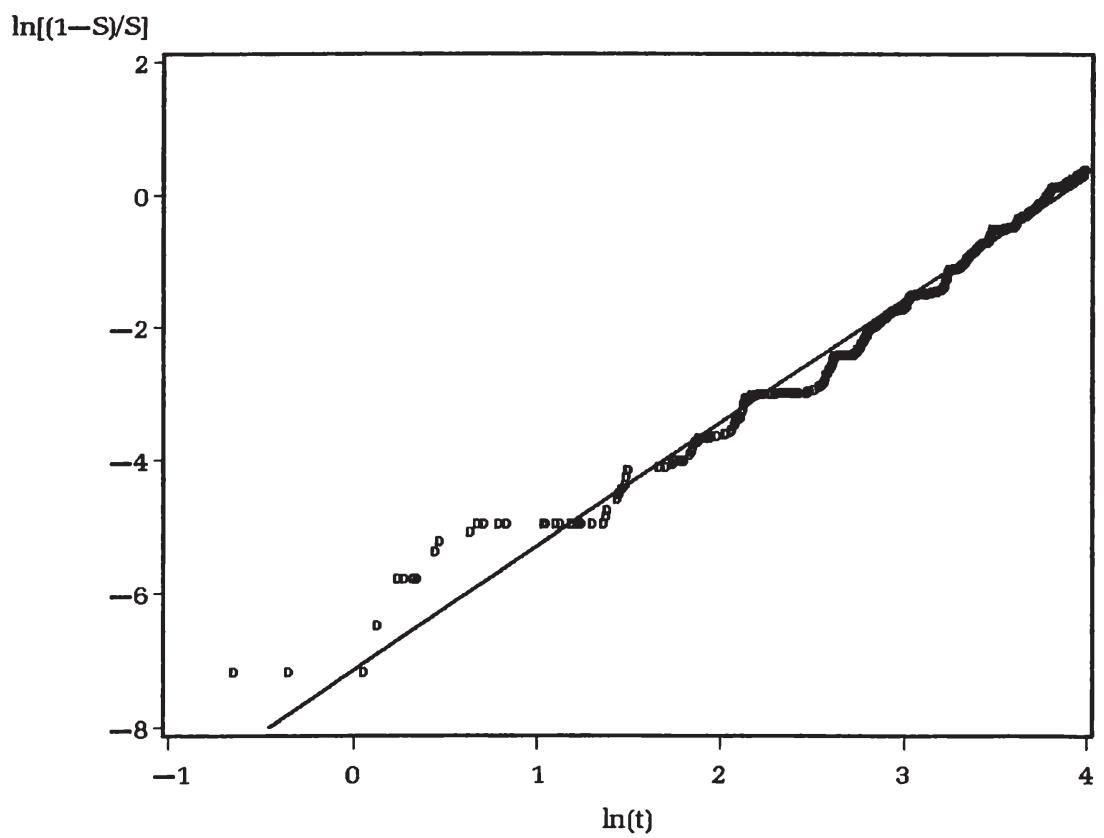

Abbildung 12.1: Graphische Überprüfung der log-logistischen Verteilungsannahme für die Reststudiendauer $t_{1}$ 
Das Weibull-Modell zeigt bei diesem graphischen Verfahren eine ähnlich gute Anpassung wie das log-logistische Modell, das log-normale und das ExponentialModell eine etwas schlechtere Anpassung an die Daten. Die Modellüberprüfungen sind Hinweis dafür, dass die getroffene Annahme einer log-logistischen Verteilung der Reststudiendauer (ohne Einbeziehung von erklärenden Variablen) passend ist.

Die ML-Schätzung führt zu folgenden Parameter-Schätzern im log-logistischen Modell: $\hat{\lambda}=\exp \left[-\hat{\beta}_{0}\right]=\exp [-3.82]=0.02 ; \hat{\sigma}=0.51$. Die geschätzte (übergangs spezifische) Hazardrate für den Studienabschluss lässt sich somit wie folgt anschreiben:

$$
h_{1}\left(t_{1}\right)=\frac{0,02 * 1,96\left(0,02 t_{1}\right)^{0,96}}{1+\left(0,02 t_{1}\right)^{1,96}}
$$

Die Reststudiendauer von Studienabbrechern (nach dem achten Semester) und von (zum Stichtag noch) Studierenden geht als rechts-zensierte Zeit in die ML-Schätzung ein. Abbildung 12.2 zeigt die geschätzte Hazardrate für den Studienabschluss bei Annahme eines log-logistischen Modells. Bei der Graphik ist $\mathrm{zu}$ beachten, dass der Ursprung der Beginn des neunten Semesters ist, da für die ersten acht Semester die übergangsspezifische Hazardrate mit Null angenommen wird.

An der Abbildung ist zu erkennen, dass die Hazardrate etwa bis zum sechzehnten Semester steigt und dann zu fallen beginnt. Es sei noch erwähnt, dass die Gestalt der Hazardfunktion bei Annahme eines verallgemeinerten GammaModells bis zum sechzehnten Semester ähnlich steigt, jedoch danach weniger steil fällt als beim log-logistischen Modell.

\section{Analyse der Reststudienzeit auf Basis der Stammdaten aus der Hörerevidenz - Basis-Modell}

Im darauffolgenden Schritt der Modellentwicklung wurde die Reststudiendauer als Funktion der folgenden (im Abschnitt 10.1 definierten und im Kapitel 11 für den Jahrgang 1990 deskriptiv analysierten) zeitkonstanten Variablen aus der Hörerevidenz spezifiziert:

- Geschlecht ("sex") mit den weiblichen Studierenden als Referenzkategorie

- Alter bei der Matura (in Jahren; "matalt"); das Alter bei der Matura wird als Hilfsvariable verstanden, die annähernd angibt, ob die Schule ohne Zeitverzögerung abgeschlossen worden ist. Über diese Variable kann etwa die 


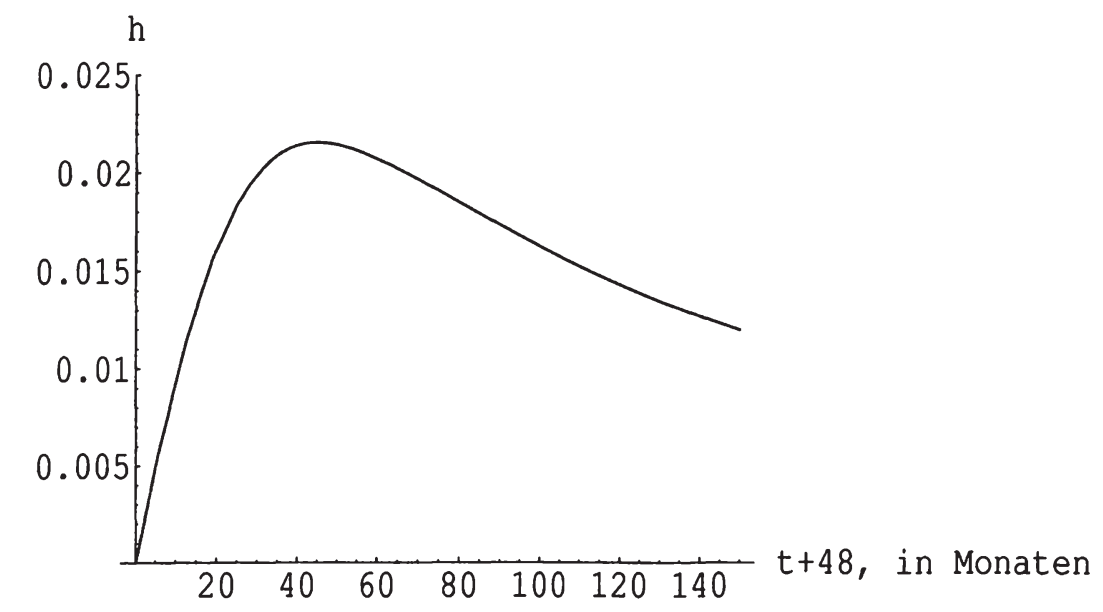

Abbildung 12.2: Geschätzte Hazardrate für den Studienabschluss bei loglogistischer Verteilungsannahme (ohne Einbeziehung von Kovariablen)

Hypothese überprüft werden, dass ein verzögerter Schulabschluss auch auf eine längere Studiendauer hinweist.

- Zeit zwischen Matura und Studienbeginn (in Monaten; "lag")

- Schultyp (AHS als Referenzkategorie); über diese Variable kann überprüft werden, ob der Abschluss eines bestimmten Schultyps Vor- bzw. Nachteile an der Wirtschaftsuniversität bringt.

- Zeitpunkt der Immatrikulation als kategorielle Variable mit den Ausprägungen "woche1" für Studierende, die in der ersten Woche der Immatrikulationszeit immatrikuliert haben (Referenzkategorie; $64 \%$ der einbezogenen Immatrikulierten des Jahrgangs 1990); "woche2"(19\%), "woche3"(8\%), "woche4" (4\%) und "woche5" (5\%) für Studierende, die in der zweiten, dritten, vierten, fünften Woche oder später immatrikuliert haben. Die Variable wurde in das Modell aufgenommen, um die Hypothese zu überprüfen, dass sich spätere Immatrikulationen negativ auf die Studiendauer auswirken. Das Immatrikulationsdatum kann als Hilfsvariable für die Zielstrebigkeit oder 
Entschlossenheit zu Studienbeginn gesehen werden. Studierende, die spät immatrikulieren, sind sich oft nicht sicher, ob bzw. welche Studienrichtung sie studieren sollen, und entscheiden sich spät.

Die Studienrichtungen, die - wie erwähnt - an der Wirtschaftsuniversität sehr ähnliche Studienpläne haben, wurden nicht in das Modell einbezogen, da die Analyse der Dauer an der Wirtschaftsuniversität (und nicht die einer Studienrichtung) im Vordergrund steht.

Für die folgenden Analysen der Reststudienzeit $t_{1}$ in Abhängigkeit der oben angeführten Variablen wird in Anlehnung an die Vorstudie in einem ersten Schritt das log-logistische Modell

$$
h\left(t_{1} \mid \mathbf{x}\right)=\frac{\lambda(\mathbf{x}) \delta\left(\lambda(\mathbf{x}) t_{1}\right)^{\delta-1}}{1+\left(\lambda(\mathbf{x}) t_{1}\right)^{\delta}}
$$

herangezogen.

Das Ausgangsmodell beinhaltet mit dem Alter bei der Matura ("matalt") und dem Zeitabstand zwischen Matura und Studienbeginn ("lag") zwei kontinuierlich skalierte Variablen. Es gilt zu überprüfen, ob die Modellierung der beiden Merkmale als kontinuierliche Variablen korrekt ist. Zur Überprüfung, ob eine kontinuierliche Variable linear auf die abhängige Variable wirkt, können Martingal-Residuen verwendet werden; siehe Abschnitt 6.5. Abbildung 12.3 zeigt die gegen die Variable "lag" aufgetragenen, (mit der LOWESS-Methode) geglätteten Martingal-Residuen, die auf Basis eines (übergangsspezifischen) log-logistischen Regressionsmodells unter Einbeziehung aller Kovariablen bis auf "lag" und "matalt" ermittelt worden sind.

Die Abbildung zeigt, dass für Werte kleiner als 18 Monate die Annahme einer linearen funktionalen Form für die Variable "lag" nicht geeignet ist, da die geglätteten Martingal-Residuen nicht die Gestalt einer Geraden haben. Die funktionelle Form der geglätteten Residuen lässt den Schluss zu, dass dieses Merkmal besser als kategorielle Variable zu modellieren ist. Eine natürliche Trennung ergibt sich, indem man in den ersten 18 Monaten zwischen Studierenden unterscheidet, die (dem "Normalfall" entsprechend) im Mai oder Juni des Immatrikulationsjahres oder des Vorjahres maturiert und jenen, die an einem anderen ("aussernatürlichen") Termin die Schule abgeschlossen haben, und eine weitere Gruppe für die wenigen Studierenden bildet, die mehr als 18 Monate zwischen Matura und Studienbeginn verstreichen haben lassen. Die Variable "lag" wird dann in folgende fünf Kategorien unterschieden:

- "lag0" als Referenzkategorie für diejenigen Studierenden, die im Mai oder 


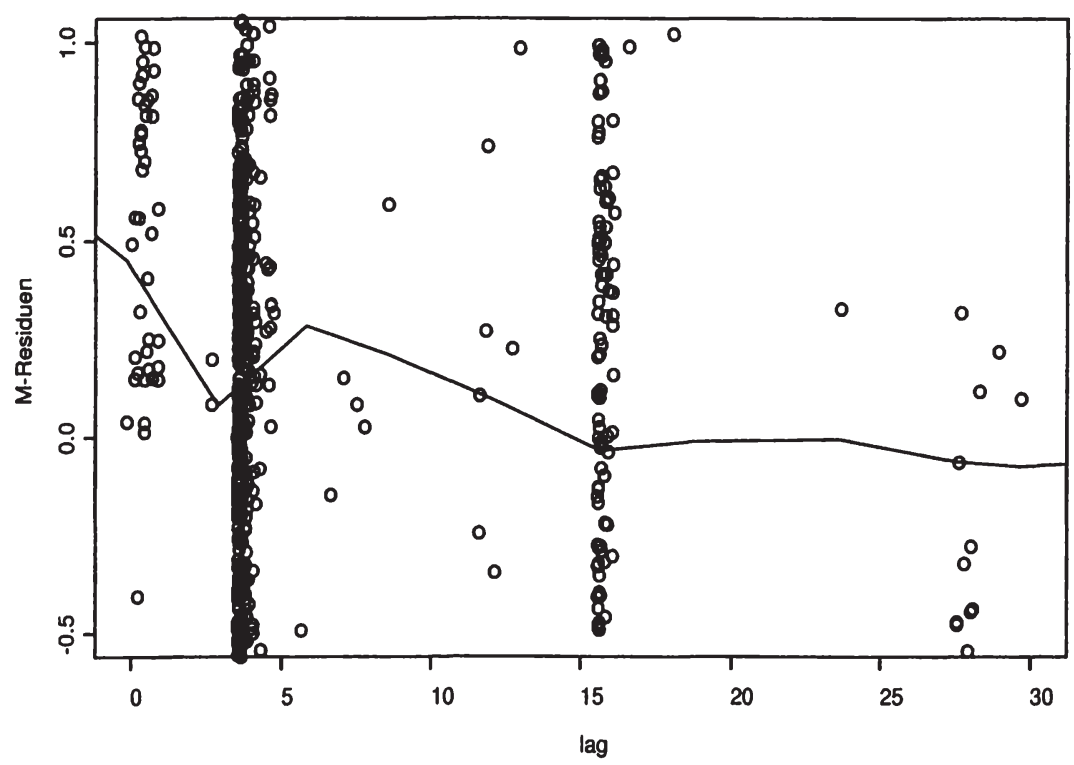

Abbildung 12.3: Plot der (geglätteten) Martingal-Residuen gegen die Variable "lag" (in Monaten; log-logistisches Modell)

Juni des Immatrikulationsjahres maturiert haben; das sind $72 \%$ der in das Modell einbezogenen Immatrikulierten des Wintersemesters 1990/91

- "lag1" für Studierende, die im September oder Oktober des Immatrikulationsjahres maturiert haben (5\%)

- "lag2" für Studierende, die vor dem Mai im Immatrikulationsjahr maturiert haben $(2 \%)$

- "lag3" für Studierende, die im Mai oder Juni des Jahres vor dem Immatrikulationsjahr maturiert haben (16\%); in diese Kategorie fallen etwa auch (männliche) Studierende, die zwischen Schulabschluss und Studienbeginn das Bundesheer absolviert haben

- "lag4" für Studierende, die mehr als 18 Monate zwischen Matura und Studienbeginn verstreichen haben lassen $(5 \%)$; in diese Kategorie fallen Studierende, die sich nach der Schule nicht sofort für ein Studium entschieden haben 
Die in der Abbildung 12.3 gezeigte funktionale Form der Variablen "lag" konnte in ergänzenden Modellüberprüfungen auch für die Immatrikulationsjahrgänge 1991 bis 1993 als typisch nachgewiesen werden.

Für die Variable "matalt" wurde ebenfalls mit Hilfe der Martingal-Residuen die funktionale Form überprüft; siehe Abbildung 12.4.

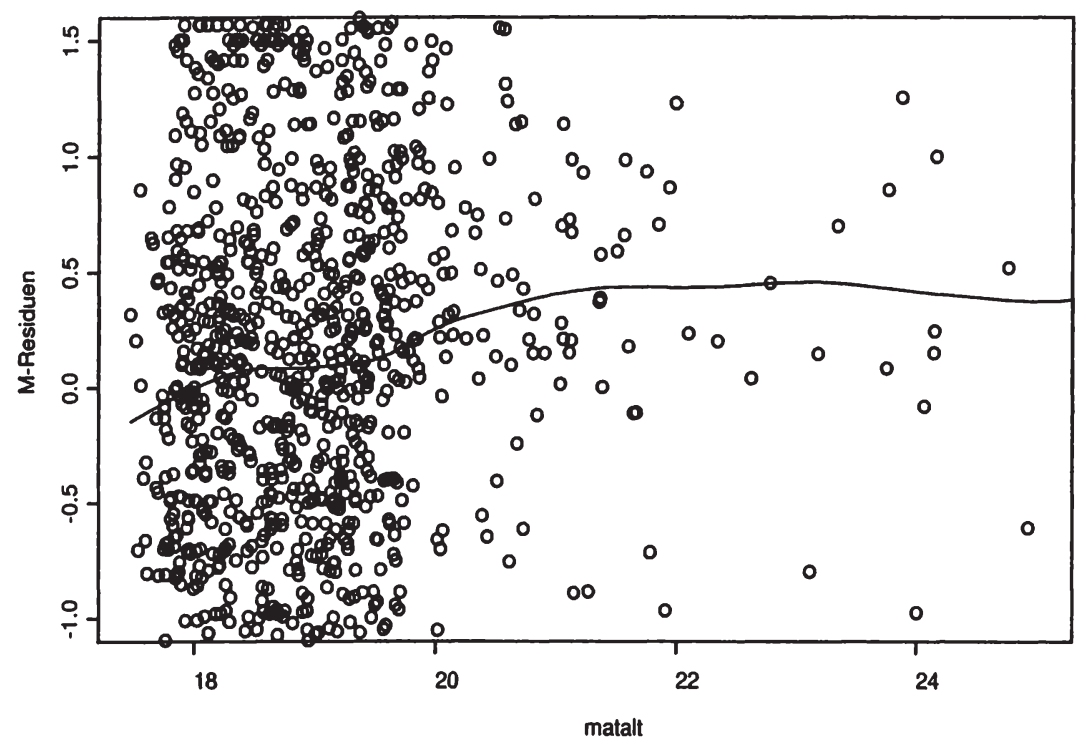

Abbildung 12.4: Plot der (geglätteten) Martingal-Residuen gegen die Variable "matalt" (log-logistisches Modell)

An dieser Abbildung ist zu erkennen, dass die Variable Alter nicht linear auf $\ln t_{1}$ einwirkt und eine Modellierung mit Hilfe einer binären Variablen geeigneter scheint. Der Abbildung entsprechend wird das Alter für die folgenden Analysen in die beiden Altersgruppen $(0 ; 20),[20, \infty)$ unterteilt ("matalt1", wobei die Altersgruppe $(0 ; 20)$ die Referenzkategorie bildet; $90 \%$ der einbezogenen Studierenden fallen in diese Kategorie). Eine ähnliche funktionale Form wurde auch für die Immatrikulationsjahrgänge 1991 bis 1993 gefunden.

Tabelle 12.2 zeigt (neben einigen Informationen zum Modell wie beispielsweise der Anzahl der in das Modell einbezogenen Studierenden) die geschätzten Parameter bei Implementierung eines log-logistischen Modells zur Analyse der Reststudiendauer unter Einbeziehung der eingangs definierten Variablen, wobei die beiden kontinuierlichen Variablen "matalt" und "lag" als kategorielle 
Merkmale implementiert worden sind. Als Referenzgruppe gelten damit weibliche Studierende mit AHS-Matura, die im Mai oder Juni des Immatrikulationsjahres maturiert haben und bei der Matura jünger als 20 Jahre alt waren.

Tabelle 12.2: Parameterschätzer des log-logistischen Modells für die Reststudiendauer auf Basis der Stammdaten (Jahrgang 1990)

Model Information

Number of Observations

Noncensored Values

Right Censored Values

Name of Distribution

Log Likelihood

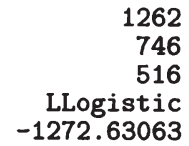

746

516

$-1272.63063$

Analysis of Parameter Estimates

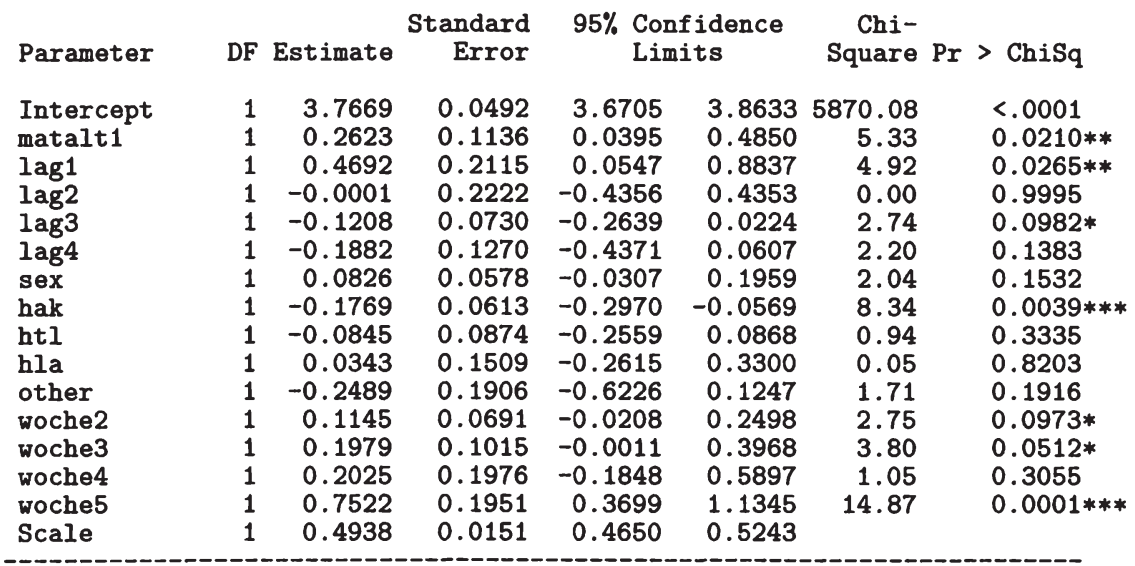

$* \mathrm{p}<0.1 ; * * \mathrm{p}<0.05 ; * * * \mathrm{p}<0.01$

Zur Überprüfung, ob das in Tabelle 12.2 implementierte log-logistische Modell eine gute Anpassung an die Daten liefert, wurde einerseits ein graphischer Test mit Hilfe der Cox-Snell-Residuen (siehe Abschnitt 5.3) durchgeführt, und andererseits der logarithmierte Wert der Likelihood-Funktion über das $A I C$ Kriterium mit den entsprechenden Werten anderer Verteilungsannahmen verglichen. Abbildung 12.5 zeigt für das log-logistische Modell eine gute Anpassung an eine Gerade, während für andere Modelle wie das Exponential-Modell eine deutliche Abweichung zu erkennen ist; siehe Abbildung 12.6. Der AICWert des log-logistischen Modells ist unter den betrachteten log-linearen Modellen am geringsten. Der Test (mit Hilfe der Likelihood-Quotienten-Statistik), dass keine der einbezogenen Variablen einen Erklärungsbeitrag liefert, führt mit einem $\mathrm{p}$-Wert $<0.0001$ zur Ablehnung. 
In einem nächsten Schritt wurden Wechseleffekte unter den Variablen - insbesondere mit dem Geschlecht - überprüft.

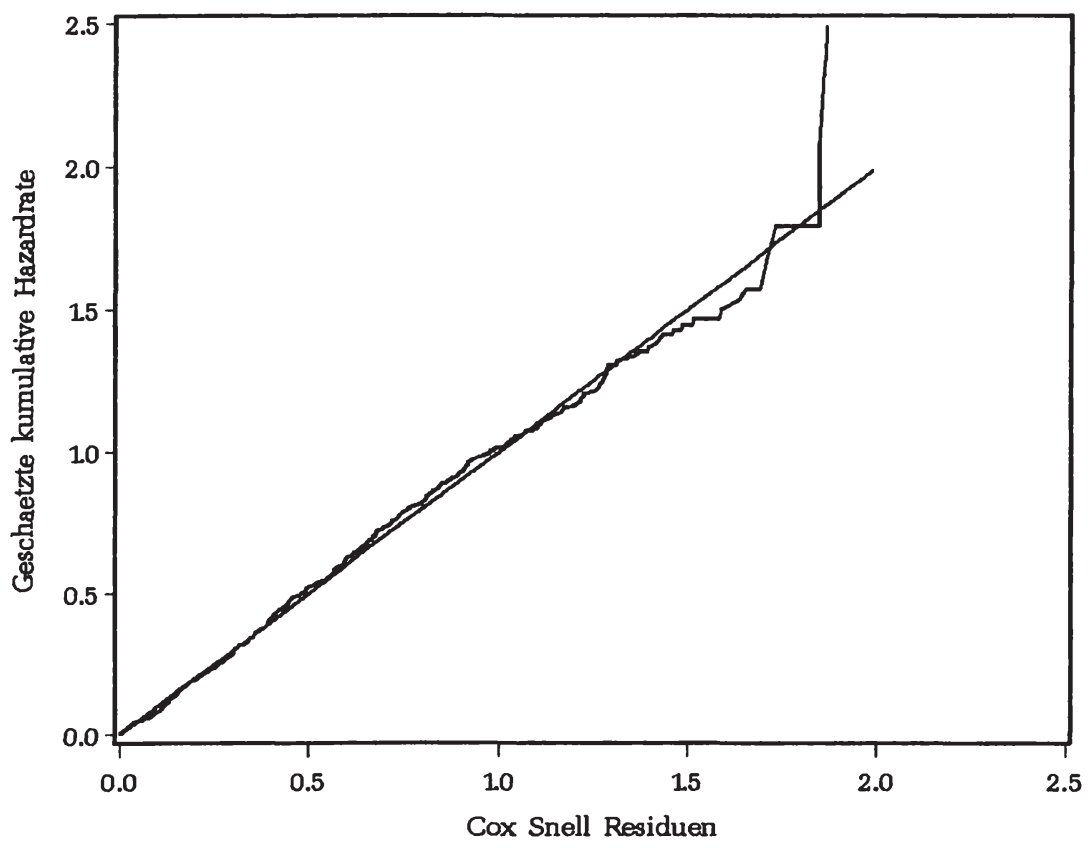

Abbildung 12.5: Plot der Cox-Snell Residuen bei Implementierung eines loglogistischen Modells für den Studienabschluss

In Tabelle 12.2 zeigt das Geschlecht - bei gleicher Ausprägung aller anderen einbezogenen Variablen - keinen Einfluss auf die Studiendauer $(p=0.15)$. Häufig ist jedoch zu beobachten, dass die Effekte der einbezogenen Kovariablen zwischen den Geschlechtern differieren. Daher können entweder Modelle für Studentinnen und Studenten getrennt geschätzt (vgl. Booth und Satchell (1995) oder Smith und Naylor (2001)) oder in das gemeinsame Modell ergänzend Interaktionsterme zwischen dem Geschlecht und den einbezogenen Variablen aufgenommen werden.

Bei der Implementierung solcher Interaktionsvariablen zeigen die Wechselwirkungen des Geschlechts mit dem Schultyp AHS und mit der zweiten Immatrikulationswoche einen Effekt auf die Studiendauer ( $p$-Werte kleiner als 0.1). Durch das Einbeziehen dieser Variablen ändern sich die Parameterschätzer der Haupteffekte, also der Schultypen, der zweiten Immatrikulationswoche und des Geschlechts; die Regressionskoeffizienten der anderen Variablen bleiben prak- 


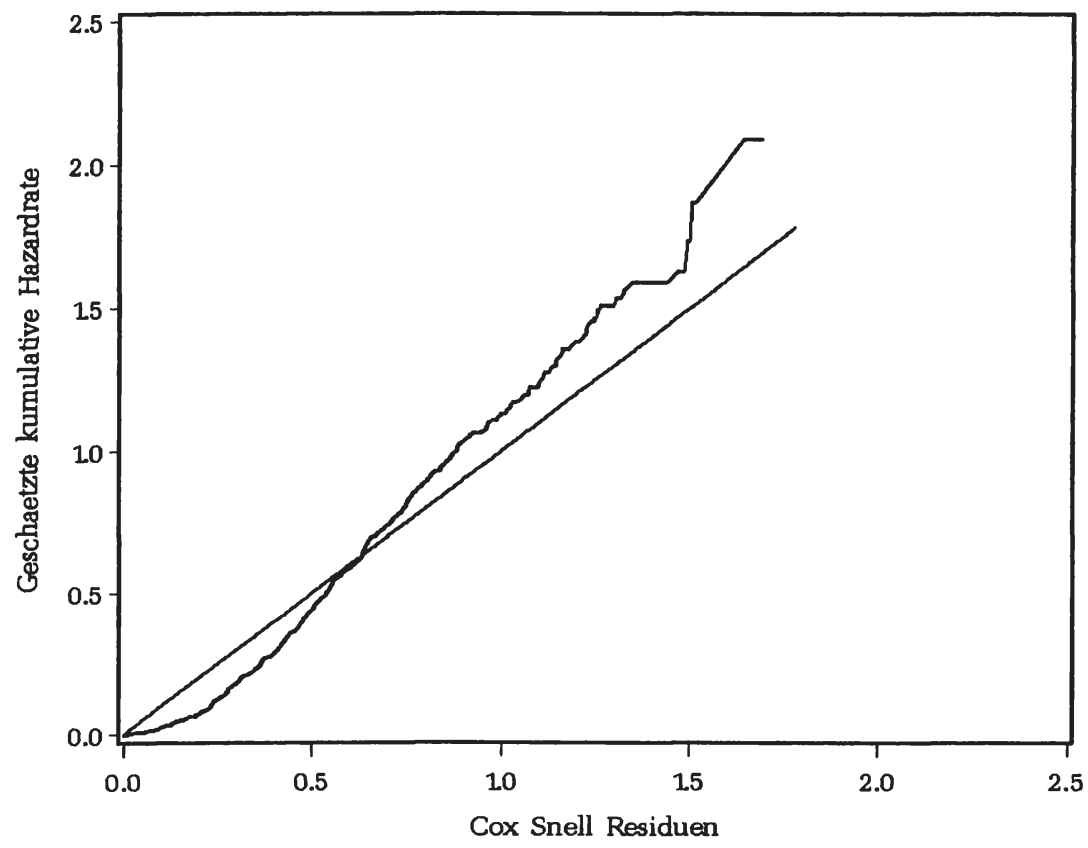

Abbildung 12.6: Plot der Cox-Snell Residuen bei Implementierung eines Exponential-Modells für den Studienabschluss

tisch unverändert. Weitere bedeutende Wechselwirkungen konnten nicht beobachtet werden. Tabelle 12.3 zeigt die Parameterschätzer des Modells nach Einbeziehung der beiden Interaktionsvariablen mit dem Geschlecht. Es sei erwähnt, dass die separate Schätzung des Modells je Geschlecht ähnliche Ergebnisse liefert.

In einem letzten Schritt der Modellentwicklung kann noch die Anzahl der Variablen durch eine schrittweise Auswahl bzw. durch Zusammenfassen von Variablen, bei denen ein Test auf Gleichheit der Regressionskoeffizienten nicht abgelehnt worden ist, verringert werden; "woche34" fasst die Variablen "woche3" und "woche4" zusammen und "lag34" die Variablen "lag3" und "lag4". Das daraus resultierende Modell (siehe Tabelle 12.4) hat keinen geringeren Erklärungsgrad als das Modell, das alle einbezogenen Variablen berücksichtigt $(p=0.85)$; die entsprechende Likelihood-Quotienten-Statistik ist $\chi^{2}(7)$ verteilt. 
Tabelle 12.3: Parameterschätzer unter Berücksichtigung von Interaktionvariablen mit dem Geschlecht (Jahrgang 1990; log-logistisches Modell)

Log Likelihood

$-1268.410831$

Analysis of Parameter Estimates

\begin{tabular}{|c|c|c|c|c|c|c|c|}
\hline Parameter & $\mathrm{DF}$ & Estimate & $\begin{array}{r}\text { Standard } \\
\text { Error }\end{array}$ & \multicolumn{2}{|c|}{$\begin{array}{l}\text { 95\% Confidence } \\
\text { Limits }\end{array}$} & $\begin{array}{r}\text { Chi- } \\
\text { Square }\end{array}$ & - > ChiSq \\
\hline Intercept & 1 & 3.6910 & 0.0556 & 3.5820 & 3.8001 & 4399.88 & $<.0001$ \\
\hline $\begin{array}{l}\text { matal } \\
\text { lag1 }\end{array}$ & $\begin{array}{l}1 \\
1\end{array}$ & $\begin{array}{l}0.2730 \\
0.4535\end{array}$ & $\begin{array}{l}0.1131 \\
0.2101\end{array}$ & 0.0416 & $\begin{array}{l}0.4958 \\
0.8653\end{array}$ & $\begin{array}{l}5.77 \\
4.66\end{array}$ & $\begin{array}{l}0.0163 * * \\
0.0309 * *\end{array}$ \\
\hline $\operatorname{lag} 2$ & 1 & 0.0210 & 0.2204 & -0.4110 & 0.4530 & 0.01 & 0.9240 \\
\hline $\operatorname{lag} 3$ & 1 & -0.1122 & 0.0729 & -0.2552 & 0.0307 & 2.37 & 0.1239 \\
\hline $\operatorname{lag} 4$ & 1 & -0.1757 & 0.1274 & -0.4254 & 0.0739 & 1.90 & 0.1678 \\
\hline $\operatorname{sex}$ & 1 & -0.0143 & 0.0912 & -0.1930 & 0.1644 & 0.02 & 0.8754 \\
\hline sex*ahs & 1 & 0.2452 & 0.1124 & 0.0250 & 0.4655 & 4.76 & $0.0291 * *$ \\
\hline hak & 1 & -0.0640 & 0.0803 & -0.2214 & 0.0933 & 0.64 & 0.4251 \\
\hline htl & 1 & 0.0739 & 0.1186 & -0.1586 & 0.3063 & 0.39 & 0.5334 \\
\hline hla & 1 & 0.0892 & 0.1520 & -0.2086 & 0.3871 & 0.34 & 0.5570 \\
\hline other & 1 & -0.1043 & 0.2014 & -0.4991 & 0.2904 & 0.27 & 0.6044 \\
\hline sex*woc & 1 & -0.2623 & 0.1403 & -0.5372 & 0.0126 & 3.50 & $0.0614 *$ \\
\hline woche2 & 1 & 0.2722 & 0.1109 & 0.0547 & 0.4896 & 6.02 & $0.0142 * *$ \\
\hline woche3 & 1 & 0.1970 & 0.1010 & -0.0009 & 0.3949 & 3.81 & $0.0511 *$ \\
\hline oche4 & 1 & 0.2147 & 0.1962 & -0.1699 & 0.5994 & 1.20 & 0.2739 \\
\hline woche5 & 1 & 0.7448 & 0.1937 & 0.3653 & 1.1244 & 14.79 & $0.0001 * * *$ \\
\hline Scale & 1 & 0.4920 & 0.0151 & 0.4633 & 0.5224 & & \\
\hline
\end{tabular}

$* \mathrm{p}<0.1 ; * * \mathrm{p}<0.05 ; * * * \mathrm{p}<0.01$

\section{Interpretation und Diskussion der Ergebnisse des Basis- Modells (Jahrgang 1990)}

Die Parameterschätzer des finalen Basis-Modells für den Jahrgang 1990 (siehe Tabelle 12.4), das die Reststudiendauer in Abhängigkeit der zu Studienbeginn in der Hörerevidenz gespeicherten Stammdaten analysiert, können wie folgt interpretiert werden:

- "matalt1": Studienanfänger, die bei der Matura älter als 20 Jahre sind, erwarten eine um etwa $32 \%(=\exp (0.28)-1)$ höhere (mediane) Reststudiendauer (= Studienzeit über der Mindeststudiendauer) als Studierende der Referenzkategorie (bei der Matura jünger als 20 Jahre und sonst gleiche Ausprägungen der anderen Variablen); $p=0.013$.

Das Alter bei der Matura kann - wie erwähnt - als eine Variable interpretiert werden, die die Studierenden darin unterscheidet, ob sie die Schule ohne Zeitverzögerung absolviert haben oder nicht. Studierende, die die Schule 
Tabelle 12.4: Parameterschätzer des finalen log-logistischen Modells für die Reststudiendauer auf Basis der Stammdaten (Jahrgang 1990)

$-1270.137408$

Analysis of Parameter Estimates

\begin{tabular}{|c|c|c|c|c|c|c|c|}
\hline \multirow{4}{*}{$\begin{array}{l}\text { Parameter } \\
\text { Intercept } \\
\text { matalt1 } \\
\text { lag1 } \\
\text { lag34 }\end{array}$} & \multirow{2}{*}{$\begin{array}{c}\text { DF } \\
1\end{array}$} & Estimate & $\begin{array}{r}\text { Standard } \\
\text { Error }\end{array}$ & \multicolumn{2}{|c|}{$95 \%$ Confidence } & Chi- & $>$ ChiSq \\
\hline & & $\begin{array}{l}3.6761 \\
0.2795\end{array}$ & $\begin{array}{l}0.0432 \\
0.1122\end{array}$ & $\begin{array}{l}3.5914 \\
0.0596\end{array}$ & & $\begin{array}{r}7228.17 \\
6.21\end{array}$ & $\begin{array}{l}<.0001 \\
0.0127 * *\end{array}$ \\
\hline & 1 & 0.4637 & 0.1688 & 0.1328 & 0.7946 & 7.54 & $0.0060 * * *$ \\
\hline & 1 & -0.1270 & 0.0658 & -0.2559 & 0.0019 & 3.73 & $0.0534 *$ \\
\hline sex & 1 & -0.0029 & 0.0712 & -0.1424 & 0.1366 & 0.00 & 0.9677 \\
\hline sex*ahs & 1 & 0.2566 & 0.0741 & 0.1113 & 0.4019 & 11.98 & $0.0005 * * *$ \\
\hline sex*woche2 & 1 & -0.2733 & 0.1398 & -0.5473 & 0.0006 & 3.82 & $0.0505 * *$ \\
\hline woche2 & 1 & 0.2693 & 0.1112 & 0.0514 & 0.4872 & 5.87 & $0.0154 * *$ \\
\hline woche34 & 1 & 0.1965 & 0.0918 & 0.0167 & 0.3764 & 4.59 & $0.0322 * *$ \\
\hline woche5 & 1 & 0.7235 & 0.1850 & 0.3609 & 1.0862 & 15.29 & $<.0001 * * *$ \\
\hline Scale & 1 & 0.4930 & 0.0151 & 0.4643 & 0.5235 & & \\
\hline
\end{tabular}

$* \mathrm{p}<0.1 ; * * \mathrm{p}<0.05 ; * * * \mathrm{p}<0.01$

ohne (oder nur mit geringem) Zeitverlust absolvieren, haben somit eine geringere zu erwartende Studiendauer.

- "lag1, lag34": Immatrikulierte, die zum ersten Matura-Nachtermin im September bzw. Oktober angetreten sind ("lag1"), erwarten eine um etwa $58 \%$ $(=\exp (0.46)-1)$ höhere Reststudiendauer als die Referenzkategorie (unter gleicher Ausprägung aller anderen Variablen); entsprechend ist auch die zusammengefasste Kategorie "lag34" zu interpretieren.

90\% der Studierenden der Kategorie "lag1" immatrikulierten (aufgrund ihres Matura-Termins) erst in der vierten oder fünften Woche der Inskriptionszeit und haben damit - wie bei der Interpretation der Variablen "woche2" bis "woche5" erläutert wird - einen ersten Startnachteil. Die Variable "lag1" korreliert somit mit den Ausprägungen "woche4" und "woche5" hoch. Lässt man etwa die Variable "lag1" in Tabelle 12.3 weg, so verringert sich der $p$ Wert der Variablen "woche4" bei einem Koeffizienten von 0.51 auf 0.0005 und der Regressionskoeffizient von "woche5" steigt von 0.75 auf 0.88 .

- "woche2, woche34, woche5": Eine spätere Immatrikulation lässt eine längere Studiendauer erwarten. Für Studienanfänger, die in der fünften Immatrikualtionswoche oder später immatrikuliert haben, liegt die erwartete Reststudiendauer $105 \%(=\exp (0.72)-1)$ über der Reststudiendauer von Studierenden, die in der ersten Woche immatrikuliert haben. 
Eine Erklärung für die positiven Regressionskoeffizienten der Immatrikulationswochen, die - wie erwähnt - bei Weglassen der Variablen "lag1" alle einen $p$-Wert kleiner als 0.1 haben, kann in dem aus einer späteren Immatrikulation entstehenden Startnachteil vermutet werden. Umso später Studierende immatrikulieren, desto weniger Zeit bleibt etwa, sich

- beraten und eine "Stundenplan" für das erste Semester erstellen zu lassen,

- auf der Universität zurecht zu finden und

- für Lehrveranstaltungen anzumelden; in manchen Lehrveranstaltungen gibt es überhaupt keine freien Plätze mehr.

An dem Projekt "Studieren in Teams", das die Eingangsphase auf der Wirtschaftsuniversität erleichtern soll, können etwa auch nur Studierende, die in den ersten drei Immatrikulationswochen immatrikuliert haben, teilnehmen. Es ist zu vermuten, dass sich eine späte Immatrikulation negativ auf die Leistungen im ersten Studienjahr auswirkt und dadurch eine längere Studiendauer zur Folge hat. Deskriptive Analysen bestätigen, dass der Immatrikultionszeitpunkt mit den Leistungen im ersten Studienjahr hoch korreliert - die Anzahl der (positiven) Leistungen im ersten Studienjahr nimmt mit der Immatrikulationswoche linear ab. Im nächsten Unterabschnitt wird dann gezeigt, dass die Leistungen im ersten Studienjahr einen hohen Erklärungsbeitrag für die Gesamtstudiendauer liefern.

- "sex*ahs": Die Interaktionsvariable gibt an, dass (männliche) AHS-Maturanten eine um $30 \%(=\exp (0.257-0.003)-1)$ längere geschätzte Reststudiendauer als AHS-Maturantinnen aufweisen. Der Schultyp AHS wirkt sich somit je nach Geschlecht unterschiedlich auf die Studiendauer aus.

Die Ausprägung "hak", die vor der Einbeziehung der Interaktionsvariablen "sex*ahs" mit einem $p$-Wert kleiner als 0.01 eine verringernde Wirkung auf die Studiendauer gezeigt hat (siehe Tabelle 12.2), weist danach mit einem $p$ Wert von 0.43 keinen Einfluss mehr auf die Studiendauer auf (siehe Tabelle 12.3). Sowohl HAK- als auch HTL-, HLA- und sonstige Absolventen unterscheiden sich nicht mehr von der Referenzkategorie, den weiblichen AHSAbsolventen ( $p$-Werte größer als 0.4). Männliche AHS-Absolventen hingegen brauchen (bei gleicher Ausprägung der anderen Variablen) länger als AHS-Absolventinnen und Absolventen der anderen Schultypen.

- "sex*woche2": Die Interaktionsvariable zeigt, dass der (die Studiendauer verlängernde) Einfluss einer Immatrikulation in der zweiten Woche für (männliche) Studenten schwächer ausgeprägt ist als für Studentinnen. 
Zusammenfassend kann geschlossen werden, dass (die Stammdaten aus der Hörerevidenz betreffend) ein zügiger Schul-Abschluss und frühes Immatrikulieren einen positiven Effekt auf die Studiendauer haben und männliche AHSAbsolventen eher länger für das Studium brauchen.

\section{Ergebnisse für die Analyse der Reststudiendauer von Im- matrikulationsjahrgängen 1989 bis 1993}

Die in den vorherigen Unterabschnitten durchgeführten Schritte der Modellentwicklung und Analyse für den Jahrgang 1990 wurden auch ergänzend für die Immatrikulationsjahrgänge der Wintersemester 1989 bis 1993 gemeinsam durchgeführt. Ziel dieser Analysen ist die Beantwortung der Frage, ob die für den Jahrgang 1990 erzielten Ergebnisse auch für andere Jahrgänge gültig sind. Tabelle 12.5 zeigt - ohne Rücksichtnahme auf eventuelle Jahrgangsspezifika die Ergebnisse für das Modell bei Annahme einer log-logistischen Verteilung.

Beim Vergleich der Tabellen 12.5 und 12.3 zeigt sich, dass fast alle Parameterschätzer im Vorzeichen und auch in der Größenordnung annähernd gleich sind - insbesondere die Variablen mit einem bedeutenden Einfluss. Die Parameterschätzer aus Tabelle 12.5 fallen alle mit Ausnahme von "woche5" in das 95\%-Konfidenzintervall der Parameterschätzer aus Tabelle 12.3. Der Effekt der Kategorie "woche5" zeigt sich etwas abgeschwächt. Weiters ist zu erwähnen, dass der Koeffizient der Variablen Geschlecht bei Betrachtung mehrerer Jahrgänge positiv (mit $p<0.01$ ) ist und somit mit einer längeren Studiendauer für männliche Immatrikulierte zu interpretieren ist. Die Interpretation der restlichen Variablen ist wie für den Jahrgang $1990 \mathrm{zu}$ führen. In einem weiteren Schritt ist es wieder möglich, (Kategorien von) Variablen zusammenzufassen bzw. wegzulassen.

Über binäre Variablen für jeden Jahrgang ("jhg89" bis "jhg93", mit dem Jahrgang 1990 als Referenzkategorie) kann ergänzend der Frage nachgegangen werden, ob sich die einzelnen Jahrgänge in der Studiendauer unterscheiden. Tabelle 12.6 zeigt die Regressionskoeffizienten dieser Dummy-Variablen bei Erweiterung des Modells aus Tabelle 12.5. Die Parameterschätzer der ursprünglich einbezogenen Variablen bleiben praktisch unverändert und sind daher nicht aufgelistet.

Der Likelihood-Quotiententest der Nullhypothese, dass die vier neu einbezogenen Variablen keinen Erklärungsbeitag liefern, führt mit $p=0.042$ zur Ablehnung. Betrachtet man in Tabelle 12.6 die vier Jahrgangs-Variablen einzeln, so zeigt sich, dass sich bei gleicher Ausprägung aller anderen Variablen lediglich 
Tabelle 12.5: Parameterschätzer des log-logistischen Modells der Reststudiendauer für die Jahrgänge 1989 bis 1993 auf Basis der Stammdaten

\section{Model Information}

Number of Observations

Noncensored Values

Right Censored Values

Name of Distribution

Log Likelihood
5694

3103

2591

LLogistic

$-5531.961201$

Analysis of Parameter Estimates

\begin{tabular}{|c|c|c|c|c|c|c|c|}
\hline Parameter & $\mathrm{DF}$ & Estimate & $\begin{array}{r}\text { Standard } \\
\text { Error }\end{array}$ & \multicolumn{2}{|c|}{$\begin{array}{l}\text { 95\% Confidence } \\
\text { Limits }\end{array}$} & \multicolumn{2}{|c|}{$\begin{array}{l}\text { Chi- } \\
\text { Square Pr > ChiSq }\end{array}$} \\
\hline Intercept & 1 & 3.6392 & 0.0274 & 3.5855 & 3.6929 & 17641.8 & $<.0001$ \\
\hline matalt1 & 1 & 0.2916 & 0.0484 & 0.1967 & 0.3865 & 36.26 & $<.0001 * * *$ \\
\hline $\operatorname{lag} 1$ & 1 & 0.3308 & 0.0941 & 0.1465 & 0.5151 & 12.37 & $0.0004 * * *$ \\
\hline lag2 & 1 & 0.2208 & 0.0963 & 0.0322 & 0.4095 & 5.26 & $0.0218 * *$ \\
\hline $\operatorname{lag} 3$ & 1 & -0.1103 & 0.0351 & -0.1790 & -0.0416 & 9.90 & $0.0017 * * *$ \\
\hline lag4 & 1 & -0.0993 & 0.0559 & -0.2089 & 0.0103 & 3.15 & $0.0759 *$ \\
\hline sex & 1 & 0.1461 & 0.0406 & 0.0666 & 0.2256 & 12.97 & $0.0003 * * *$ \\
\hline sex*ahs & 1 & 0.0925 & 0.0518 & -0.0090 & 0.1940 & 3.19 & $0.0739 *$ \\
\hline hak & 1 & -0.0971 & 0.0379 & -0.1714 & -0.0229 & 6.57 & $0.0104 * *$ \\
\hline htl & 1 & 0.0438 & 0.0526 & -0.0593 & 0.1469 & 0.69 & 0.4048 \\
\hline hla & 1 & 0.0392 & 0.0663 & -0.0908 & 0.1692 & 0.35 & 0.5544 \\
\hline other & 1 & 0.0503 & 0.0885 & -0.1232 & 0.2238 & 0.32 & 0.5698 \\
\hline sex*woche2 & 1 & -0.1526 & 0.0620 & -0.2742 & -0.0310 & 6.05 & $0.0139 * *$ \\
\hline woche2 & 1 & 0.2528 & 0.0467 & 0.1611 & 0.3444 & 29.23 & $<.0001 * * *$ \\
\hline woche3 & 1 & 0.2021 & 0.0497 & 0.1047 & 0.2995 & 16.54 & $<.0001 * * *$ \\
\hline woche4 & 1 & 0.2537 & 0.0868 & 0.0836 & 0.4238 & 8.54 & $0.0035 * * *$ \\
\hline พoche5 & 1 & 0.3372 & 0.0883 & 0.1642 & 0.5102 & 14.59 & $0.0001 * * *$ \\
\hline Scale & 1 & 0.4887 & 0.0074 & 0.4745 & 0.5034 & & \\
\hline
\end{tabular}

$* \mathrm{p}<0.1 ; * * \mathrm{p}<0.05 ; * * * \mathrm{p}<0.01$

der Jahrgang 1993 in der Studiendauer vom Jahrgang 1990 geringfügig unterscheidet. Die erwartete Reststudiendauer wird für den Jahrgang 1993 um 9\% geringer als für den Jahrgang 1990 geschätzt. Über Interaktionsvariablen bzw. eine getrennte Analyse der Jahrgänge kann zusätzlich untersucht werden, ob der Einfluss der einbezogenen Variablen aus der Hörerevidenz zwischen den Jahrgängen variiert. Es konnte nichts Auffälliges beobachtet werden.

\section{Modelle der Reststudienzeit auf Basis der Stamm- und Prüfungsdaten}

Prüfungsdaten - etwa Ablegungszeitpunkte, Noten oder wiederholte Antritte - widerspiegeln den Studienfortschritt und sind daher hoch prädiktiv für die Studiendauer (und die Abbruchrate). Um die Hypothese zu überprüfen, dass 
Tabelle 12.6: Parameterschätzer der binären Jahrgangs-Variablen (Jahrgänge 1989 bis 1993)

Log Likelihood

$-5527.020509$

Analysis of Parameter Estimates

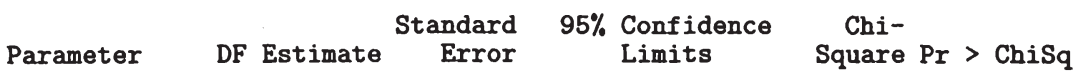

$\begin{array}{lrrrrrrr}\text { jhg89 } & 1 & -0.0136 & 0.0368 & -0.0857 & 0.0585 & 0.14 & 0.7109 \\ \text { jhg91 } & 1 & 0.0300 & 0.0375 & -0.0435 & 0.1034 & 0.64 & 0.4239 \\ \text { jhg92 } & 1 & -0.0343 & 0.0382 & -0.1093 & 0.0406 & 0.81 & 0.3691 \\ \text { jhg93 } & 1 & -0.0943 & 0.0406 & -0.1738 & -0.0148 & 5.41 & 0.0201 * *\end{array}$

$* \mathrm{p}<0.1 ; * * \mathrm{p}<0.05 ; * * * \mathrm{p}<0.01$

das Leistungsprofil des ersten Studienjahrs einen hohen prädiktiven Charakter für die Studiendauer an der Wirtschaftsuniversität hat, wurden in einem weiteren Schritt Prüfungsdaten aus dem ersten Studienjahr in das log-logistische Regressionsmodell integriert:

- Anzahl der positiven Lehrveranstaltungsprüfungen ("gesposj1") exklusive Ergänzungsprüfungen; diese Prüfungen wurden hier nicht berücksichtigt, da sie Voraussetzung aber nicht Bestandteil der Studienpläne sind. Der Mittelwert der Variablen "gesposj1" liegt für die in das Modell einbezogenen Immatrikulierten bei etwa acht positiven Prüfungen.

- Ablegen mindestens einer positiven Vor- bzw. Teildiplomprüfung als binäre Variable ("vpj1"); $9 \%$ der einbezogenen Studierenden haben mindestens eine Vor- bzw. Teildiplomprüfung im ersten Studienjahr abgelegt.

- Notendurchschnitt aller im ersten Studienjahr abgelegten Lehrveranstaltungsprüfungen ("average") exklusive Ergänzungsprüfungen; der Mittelwert der Variablen liegt bei 3.5.

Mit der Einbeziehung von Prüfungsdaten soll untersucht werden, inwieweit unter der Berücksichtigung der in der Hörerevidenz vorhandenen Information nach dem ersten Studienjahr Individualprognosen über die Studiendauer (und die Studienabbruch-Wahrscheinlichkeit) möglich sind. Diese Fragestellung gewinnt im Lichte der an der Wirtschaftsuniversität im Wintersemester 2002/2003 eingeführten Studieneingangsphase von einem Studienjahr umso mehr an Bedeutung. 
Als Datenbasis für die Modelle dieses Unterabschnitts wurden jene inländischen Immatrikulierten der Wintersemester 1989/90 bis 1993/94 herangezogen, die mindestens acht Semester an der Wirtschaftsuniversität studiert und mindestens eine Lehrveranstaltungsprüfung im ersten Studienjahr abgelegt haben. Durch die zweite Einschränkung, die sich in der Aufnahme der Variablen Notendurchschnitt begründet, geht die Information von weiteren $2 \%$ der bis zum Stichtag erfolgten Studienabschlüsse verloren. Eine alternative Modellierung, die auch die Studierenden berücksichtigt, die keine Lehrveranstaltungsprüfung im ersten Studienjahr abgelegt haben, und die Variable Notendurchschnitt nicht in das Modell einbezieht, führt zu keinen offensichtlichen Abweichungen gegenüber der gleichen Modellierung mit eingeschränkter Population.

Die Anzahl der positiven Lehrveranstaltungsprüfungen und der Notendurchschnitt sind kontinuierlich skalierte Variablen. Die Überprüfung, ob die Modellierung als kontinuierliche Variablen passend ist, erfolgte mittels MartingalResiduen.

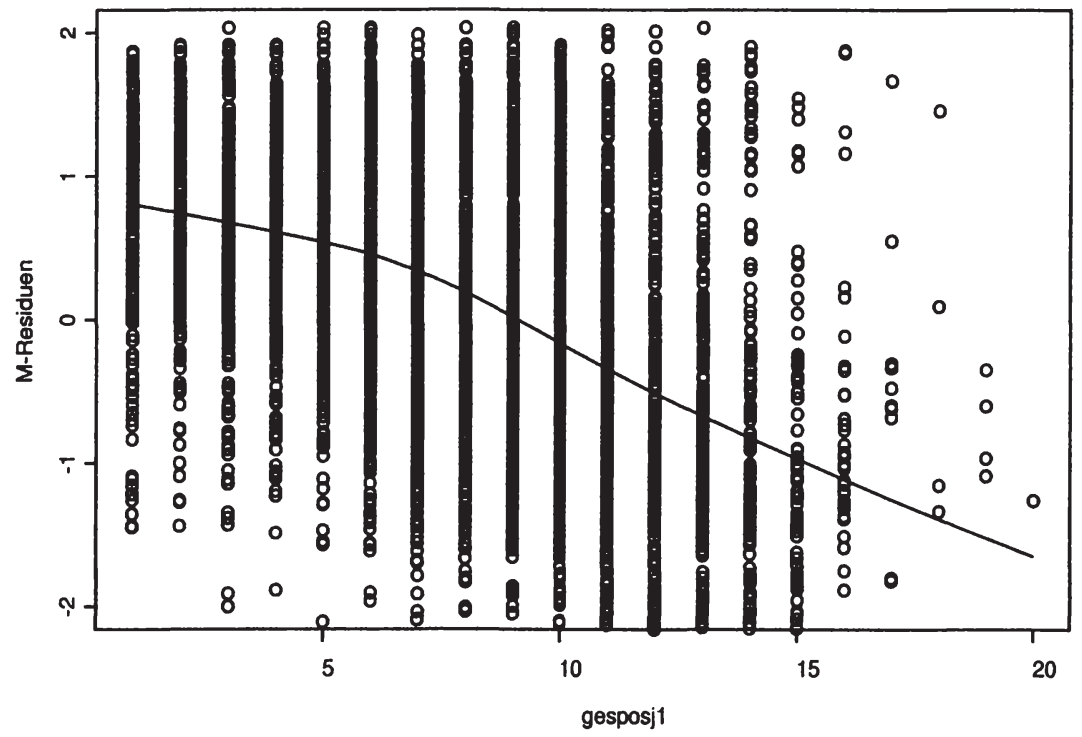

Abbildung 12.7: Plot der (geglätteten) Martingal-Residuen gegen die Variable "gesposj1" (log-logistisches Modell)

Beide Abbildungen 12.7 und 12.8 lassen erkennen, dass die Einbeziehung als kontinuierliche Variablen in Ordnung ist. Dabei muss allerdings erwähnt werden, dass die abgebildeten Martingal-Residuen auf Basis eines (übergangsspe- 


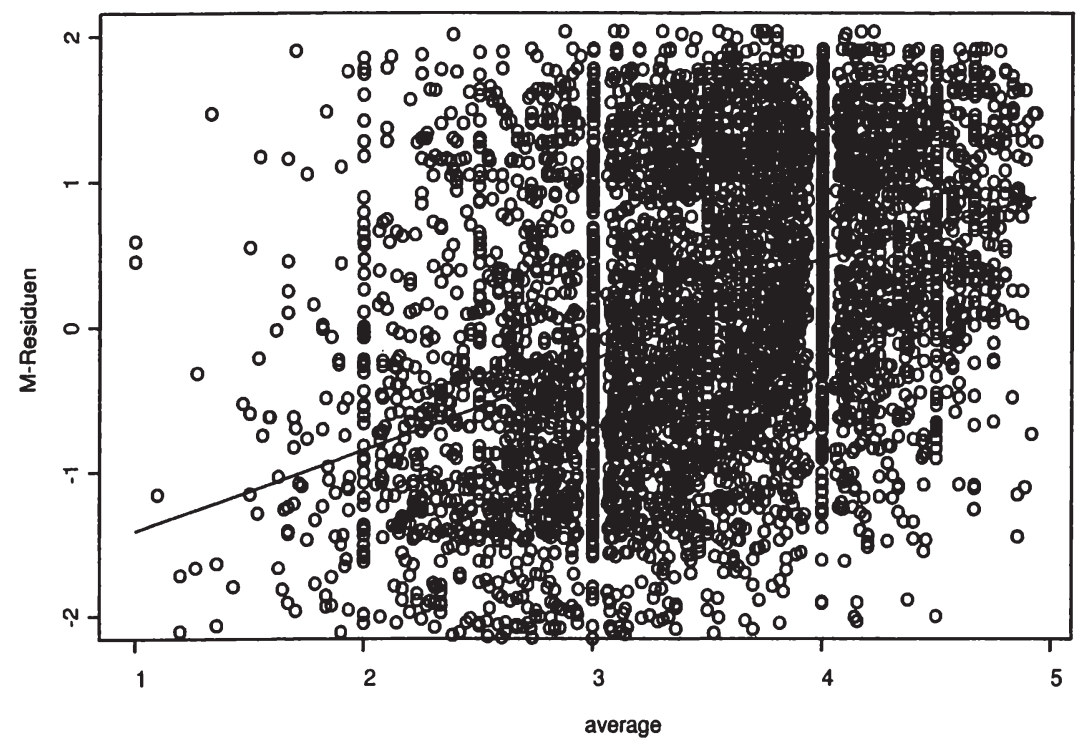

Abbildung 12.8: Plot der (geglätteten) Martingal-Residuen gegen die Variable "average" (log-logistisches Modell)

zifischen) log-logistischen Regressionsmodells unter Einbeziehung der Stammdaten und "vpj1" ermittelt worden sind. Bezieht man zusätzlich die jeweils andere Prüfungsvariable in das Modell ein, so ist etwa die lineare Form der Variablen "average" ab dem Notendurchschnitt Vier etwas gebrochen. Das bedeutet, dass unter Einbeziehung von "gesposj1" ab einem Notendurchschnitt von Vier keine wesentlichen Unterschiede in der Studiendauer zu erwarten sind.

Die folgenden Interpretationen beziehen sich auf eine Referenzgruppe von weiblichen Studierenden mit AHS-Matura, die im Mai oder Juni des Immatrikulationsjahres maturiert haben und bei der Matura jünger als 20 Jahre alt waren; sie haben im ersten Studienjahr acht Lehrveranstaltungen positiv absolviert ("gesposj1" =8), dabei eine durchschnittliche Benotung von Drei erreicht ("average"=3) und keine Vor- bzw. Teildiplomprüfung absolviert ("vpj1"=0).

Die mediane Reststudiendauer der Referenzkategorie kann (unter der Annahme eines bedingt unabhängigen Zensierungsmechanismus) über

$$
S_{1}\left(t_{1} \mid \mathbf{x}\right)=\exp \left(-\int_{0}^{t_{1}} h_{1}(u \mid \mathbf{x}) d u\right)
$$


wobei für $\mathbf{x}$ die Werte der Referenzgruppe einzusetzen sind, zu 14 Semester (sechs Semester über der Mindeststudiendauer) geschätzt werden. Tabelle 12.7 zeigt die Parameterschätzer des finalen Modells (unter Einbeziehung von Stamm- und Prüfungsdaten) für die Multiplikatoren der medianen Reststudiendauer der Referenzgruppe. Zu erwähnen ist, dass die Variablen "woche2" bis "woche5" nach Einbeziehen der Prüfungsdaten keinen Erklärungsbeitag mehr liefern und daher nicht mehr in das Modell einbezogen wurden.

Tabelle 12.7: Analyse der medianen Reststudiendauer der Jahrgänge 1989 bis 1993 auf Basis der Stamm- und Prüfungsdaten (log-logistisches Modell

\section{Model Information}

\author{
Number of Observations \\ Noncensored Values \\ Right Censored Values \\ Name of Distribution \\ Log Likelihood
}

5466

3034

2432

LLogistic $-4549.075241$

\section{Analysis of Parameter Estimates}

\begin{tabular}{|c|c|c|c|c|c|c|c|}
\hline \multirow{3}{*}{ Parameter } & DF & Estimate & $\begin{array}{r}\text { Standard } \\
\text { Error }\end{array}$ & \multicolumn{2}{|c|}{$95 \%$ Confidence } & $\begin{array}{r}\text { Chi- } \\
\text { Square }\end{array}$ & $>\mathrm{ChiSq}$ \\
\hline & 1 & 3.6892 & 0.0985 & 3.4961 & 3.8823 & 1402.07 & $<.0001$ \\
\hline & 1 & 0.1289 & 0.0412 & 0.0482 & 0.2096 & 9.80 & $0.0017 * * *$ \\
\hline $\operatorname{lag} 1$ & 1 & 0.1349 & 0.0653 & 0.0069 & 0.2628 & 4.27 & $0.0389 * *$ \\
\hline $\operatorname{lag} 4$ & 1 & -0.0908 & 0.0479 & -0.1848 & 0.0031 & 3.59 & $0.0582 *$ \\
\hline sex & 1 & 0.0846 & 0.0317 & 0.0223 & 0.1468 & 7.10 & $0.0077 * * *$ \\
\hline sex*ahs & 1 & 0.0764 & 0.0411 & -0.0042 & 0.1569 & 3.45 & $0.0632 *$ \\
\hline hak & 1 & 0.1290 & 0.0301 & 0.0700 & 0.1880 & 18.35 & $<.0001 * * *$ \\
\hline htl & 1 & 0.0926 & 0.0426 & 0.0091 & 0.1760 & 4.73 & $0.0297 * *$ \\
\hline gesposj1 & 1 & -0.0882 & 0.0042 & -0.0965 & -0.0799 & 433.05 & $<.0001 * * *$ \\
\hline vpj1 & 1 & -0.4043 & 0.0376 & -0.4781 & -0.3306 & 115.48 & $<.0001 * * *$ \\
\hline average & 1 & 0.1962 & 0.0210 & 0.1551 & 0.2373 & 87.56 & $<.0001 * * *$ \\
\hline Scale & 1 & 0.3989 & 0.0061 & 0.3872 & 0.4110 & & \\
\hline
\end{tabular}

$* \mathrm{p}<0.1 ; * * \mathrm{p}<0.05 ; * * * \mathrm{p}<0.01$

Die Parameterschätzer können wie folgt interpretiert werden:

- "matalt1": Für Studierende, die bei der Matura älter als 20 Jahre sind, und sonst die gleichen Ausprägungen der restlichen Variablen haben, ist eine um etwa $14 \%$ höhere (mediane) Reststudiendauer zu erwarten als für die Referenzgruppe, somit eine Gesamtstudiendauer von ca. 14.8 Semestern; auch nach Einbeziehen der Prüfungsdaten behält die Variable "matalt1" somit ihren Erklärungsbeitrag (wenn auch etwas abgeschwächt).

- "hak, htl": Der Parameterschätzer der Variablen "hak" weist auf eine längere 
Reststudiendauer für Studierende mit HAK-Matura hin; ohne Einbeziehung der Leistungsvariablen wurde für Studierende mit HAK-Matura eine etwas geringere Studiendauer geschätzt; vgl. Tabelle 12.5. Eine nähere Untersuchung hierzu hat gezeigt, dass Studierende mit HAK-Matura im ersten Studienjahr durchschnittlich zwei bis drei Lehrveranstaltungen (exklusive Ergänzungsprüfungen) mehr ablegen als Studierende von anderen Schultypen, und dass sie daher ohne Berücksichtigung der Prüfungsdaten eine kürzere Studiendauer zu erwarten haben. Die Ausprägung "hak" korreliert somit mit den Leistungen des ersten Studienjahres.

Ein HTL-Abschluss lässt (bei gleicher Ausprägung aller anderen Variablen) eine etwas längere Studiendauer erwarten.

- "sex, sex*ahs": Die Interpretationen dieser Variablen sind auch nach Einbeziehung der Leistungsvariablen fast gleich geblieben.

Die einbezogenen Prüfungsvariablen zeigen den erwartet hohen Einfluss auf die Studiendauer:

- "gesposj1": Eine zusätzlich positiv absolvierte Lehrveranstaltungsprüfung verringert die gesamte Studiendauer im Vergleich zur Referenzgruppe auf etwa 13.5 Semester; verringert sich die Zahl der absolvierten Lehrveranstaltungsprüfungen um eine, so erhöht sich die Studiendauer auf 14.5 Semester.

- "average": Ein um ein Grad schlechterer (besserer) Notendurchschnitt als Drei erhöht (verringert) die geschätzte Studiendauer auf über 15 (bzw. unter 13 Semester).

- "vpj1": Das Absolvieren mindestens einer Diplomprüfung im ersten Studienjahr verringert die erwartete Studiendauer.

\subsection{Analyse der Studienabschlussrate - semi- parametrische Modellierung}

Mit parametrischen und semiparametrischen Ereignismodellen werden meist ähnliche Analyseziele verfolgt: die Ermittlung von Einflussfaktoren auf die betrachtete Zeitdauer bzw. auf die Hazardrate für das Eintreffen des betrachteten Ereignisses. Während sich jedoch die Analysen mit parametrischen Regressionsmodellen häufig auf die Verteilung der betrachteten Zeitdauer konzentrieren, steht bei den flexibleren semiparametrischen Modellen die Analyse von Einflussfaktoren auf die Hazardrate im Vordergrund. 
Bei den folgenden Analysen ist zu beachten, dass das semiparametrische CoxModell linear in $\ln h(t)$ formuliert ist und die in den vorangegangenen Abschnitten verwendeten parametrischen Modelle linear in $\ln t$ sind. Die Regressionskoeffizienten von semiparametrischen Modellen sind nicht direkt vergleichbar mit den Koeffizienten von log-logistischen Modellen, da das log-logistische Modell kein proportionales Hazards-Modell ist. Vergleichbar sind die $p$-Werte und die Vorzeichen der geschätzten Parameter, die im Cox-Modell genau in die entgegengesetzte Richtung sein müssten. Die Koeffizienten des in $\ln t$ formulierten Exponential- und Weibull-Modells hingegen sind durch Wechsel des Vorzeichens und (im Weibull-Modell) Dividieren der Regressionskoeffizienten durch $\sigma$ direkt vergleichbar mit dem Cox-Modell, da beide Modelle sowohl proportionale Hazards- als auch log-lineare Modelle sind; vgl. Kapitel 5.

Da das semiparametrische Modell von Cox nur die geordneten Ereigniszeiten berücksichtigt und keine Verteilungsannahme voraussetzt, ist für die folgenden Modellierungen keine Implementierung einer Garantiezeit notwendig. Alle Studienabbrüche, die vor dem ersten Studienabschluss erfolgt sind, werden entsprechend der Konstruktion der partiellen Likelihood-Funktion bei der Schätzung der Parameter nicht berücksichtigt; siehe Abschnitt 6.2. Im Gegensatz zu den parametrischen Modellen mit der Garantiezeit von acht Semester werden jedoch jene 1\% der Studienabschlüsse berücksichtigt, die vor Ende des achten Semesters erfolgt sind.

\section{Studienabschlussrate: Effekte in Abhängigkeit der Stamm- daten aus der Hörerevidenz}

In einem ersten Schritt wurde das semiparametrische Cox-Modell für die Daten aus der Hörerevidenz der Jahrgänge 1989 bis 1993 implementiert. Die geschätzten Regressionskoeffizienten unter Berücksichtigung von Interaktionvariablen (sowie die Anzahl der Ereignisse und der zensierten Fälle und die durchgeführten Tests zur globalen Hypothese, dass alle Parameter gleich Null sind) zeigt Tabelle 12.8. Aufgrund von Modellüberprüfungen wurden die Variablen "matalt" und "lag" wieder als kategorielle Variablen entsprechend dem vorhergehenden Abschnitt implementiert.

Die Regressionskoeffizienten können (auszugsweise) wie folgt interpretiert werden:

- "matalt1": Studierende, die bei der Matura älter als 20 Jahre sind, haben zu jedem Zeitpunkt $t(\geq 42)$ bei gleichen Ausprägungen der sonstigen Variablen eine um $36 \%$ geringere Hazardrate für den Studienabschluss als die Refe- 
Tabelle 12.8: Parameterschätzer eines semiparametrischen Cox-Modells für den Studienabschluss auf Basis der Stammdaten der Jahrgänge 1989 bis 1993

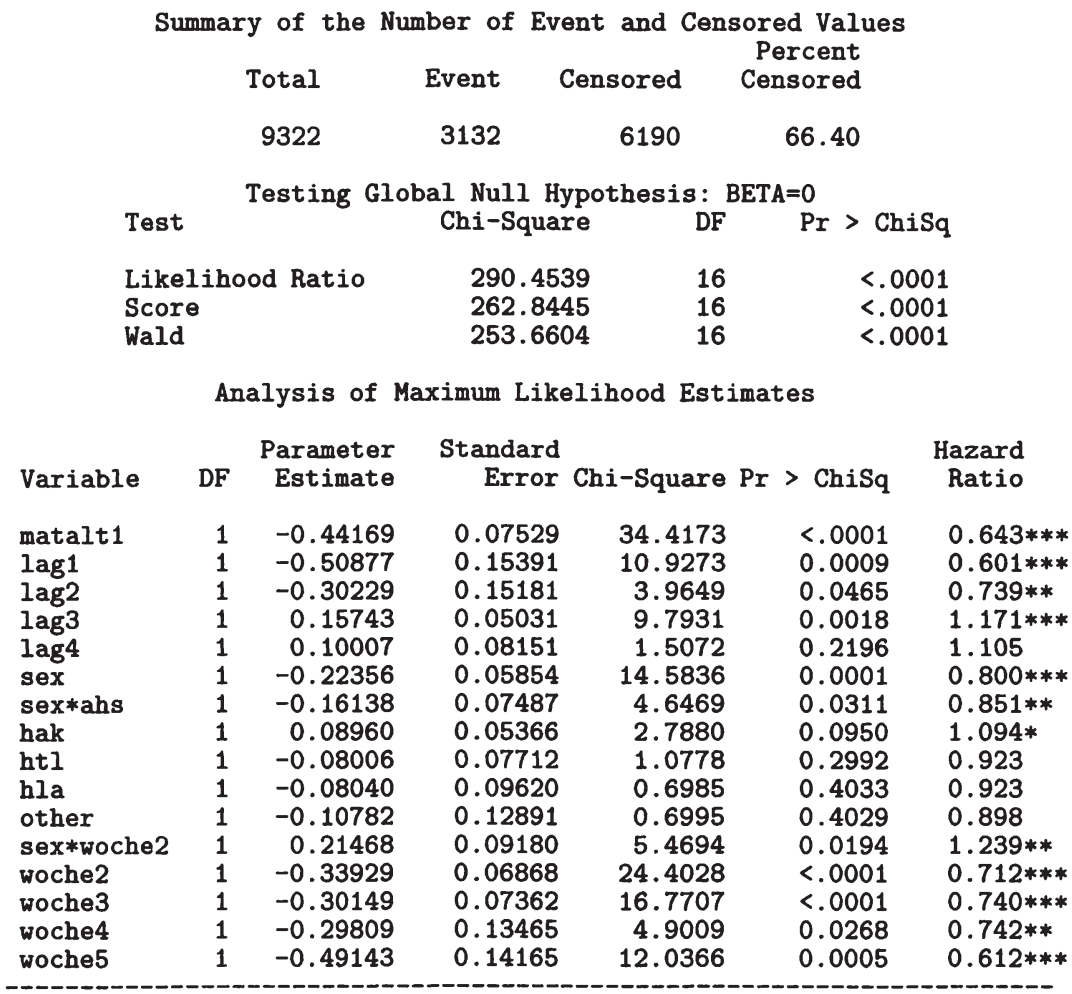

$* p<0.1 ; * * p<0.05 ; * * * p<0.01$

renzkategorie. Dieser Wert ist aus der Spalte Hazard Ratio herauszulesen $(1-0.643=0.357)$. Der $p$-Wert ist kleiner als 0.0001 .

- "lag1" bis "lag4": Immatrikulierte, die zum ersten Matura-Nachtermin im September bzw. Oktober angetreten sind ("lag1"), haben zu jedem Zeitpunkt $t(\geq 42)$ eine um $40 \%$ geringere Chance auf einen erfolgreichen Studienabschluss (unter gleicher Ausprägung aller anderen Variablen; $p=0.0009$ ); entsprechend sind auch die Variablen "lag2" bis "lag4" zu interpretieren.

- "sex": Die Studienabschlussrate von Studenten ist um $20 \%$ geringer als die von Studentinnen. 
- "hak": HAK-AbsolventInnen haben eine um $9 \%$ höhere Hazardrate für den Studienabschluss.

- "sex*ahs": Der Effekt des Schultyps AHS ist je Geschlecht unterschiedlich. Männliche AHS-Absolventen haben eine um 32\% (1- $\exp (-0.22-0.16))$ geringere Abschlussrate als AHS-Absolventinnen.

- "woche2" bis "woche5": Spätere Immatrikulationen als in der ersten Woche verringern die Hazardrate auf den Studienabschluss.

Alle Variablen liefern somit eine ähnliche Interpretation - soweit vergleichbar - wie im log-logistischen Modell.

Auf Überprüfungen der Proportionalitätsannahme im Cox-Modell wird im nächsten Unterabschnitt eingegangen.

\section{Studienabschlussrate: Effekte in Abhängigkeit von Stamm- und Prüfungsdaten}

Im nächsten Schritt wurde das semiparametrische Cox-Modell aus Tabelle 12.8 um die Prüfungsdaten des ersten Studienjahrs ("gesposj1", "vpj1", "average") erweitert. Dieses Modell berücksichtigt wieder nur Studierende, die im ersten Studienjahr mindestens eine Leistung erbracht haben. Mit Hilfe von MartingalResiduen, die auf Basis aller Stammdaten und "vpj1" ermittelt worden sind, wurde für das Cox-Modell überprüft, ob die kontinuierlich skalierten Variablen "gesposj1" und "average" linear auf $\ln h(t)$ wirken.

Abbildung 12.9 zeigt, dass der Effekt der Anzahl der Prüfungen annähernd linear ist. Die Überprüfung der linearen funktionalen Form der Variablen Notendurchschnitt ergab ebenfalls, dass die lineare funktionale Form passend ist.

Im Kapitel 6 ist erläutert worden, dass das Cox-Modell proportionale Hazardraten für unterschiedliche Ausprägungen von Kovariablen voraussetzt. In Abschnitt 6.5 wurden einige Methoden zur Überprüfung dieser Modellannahme erläutert. Zur graphischen Überprüfung der Proportionalitätsannahme einer Variablen etwa wird das Modell nach diesem Merkmal geschichtet und in Abhängigkeit der anderen Kovariablen geschätzt. Der Plot der geschichteten logarithmierten Überlebensfunktionen sollte dann annähernd parallel sein. Abbildung 12.10 zeigt diese graphische Überprüfung für die Variable "vpj1".

An der Abbildung ist zu erkennen, dass die beiden Kurven eine ähnliche Form haben, jedoch vor allem in den ersten Semestern, in denen Abschlüsse auftreten können, etwas mehr voneinander abweichen als in späteren Semestern. 


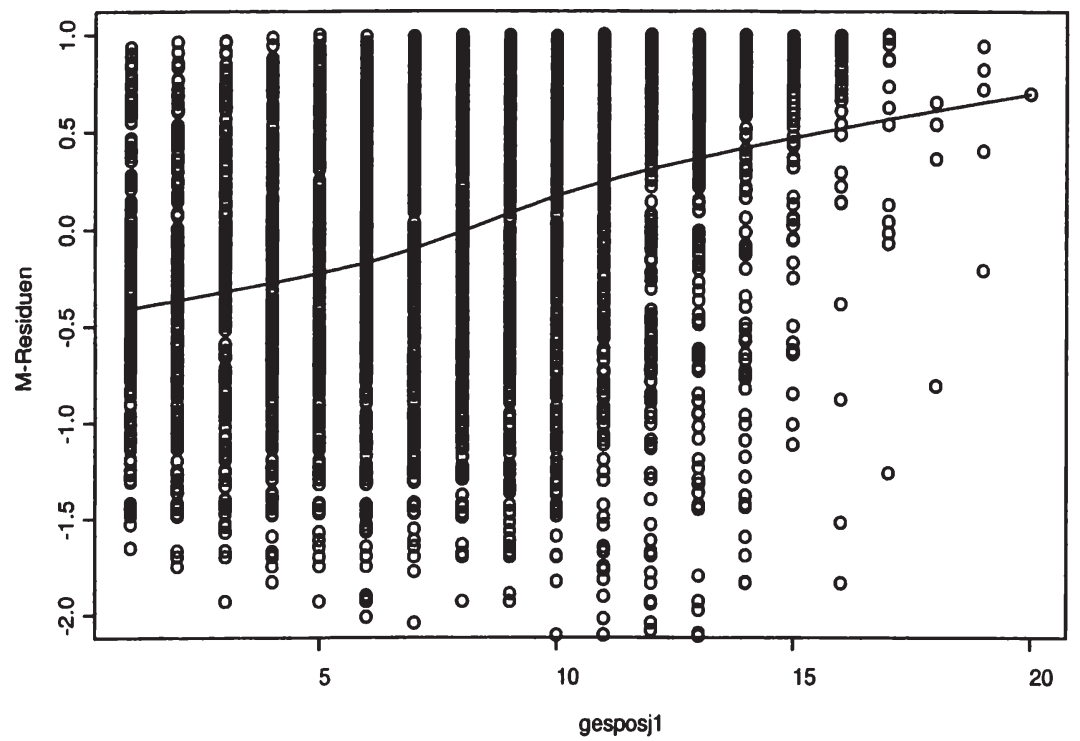

Abbildung 12.9: Plot der (geglätteten) Martingal-Residuen gegen die Variable "gesposj1" (Cox-Modell)

Dies deutet daraufhin, dass das Ablegen mindestens einer Vor- bzw. Teildiplomprüfung im ersten Studienjahr die Chance auf den Studienabschluss insbesondere zwischen dem achten und zwölften Semester erhöht, und sich dieser Unterschied danach verringert.

Will man den gleichen graphischen Test für die Variable "gesposj1" durchführen, so muss dieses (kontinuierlich skalierte) Merkmal kategorisiert werden. Eine Gruppierung der Variablen in die Kategorien 0-3 ("posj1=1"), 4-7 ("posj1=2"), 8-10 ("posj1=3") und mehr als 10 positive Leistungen im ersten Studienjahr ("posj1=4") führt zur Abbildung 12.11, wobei "posj1" die gruppierte Variable "Anzahl der positiven Leistungen im ersten Studienjahr" bezeichnet. Diese Abbildung liefert eine ähnliche Interpretation wie Abbildung 12.10.

Der gleiche Test für den Notendurchschnitt führt zur Abbildung 12.12; diese zeigt, dass die Kurven für die vier Notenkategorien $[1,2)$ ("av=1"), [2,3) ( "av=2"), $[3,4)$ ("av=3"), [4,5] ("av=4") ähnliche Form haben, jedoch in den ersten Semestern, in denen Abschlüsse auftreten können, wieder etwas weiter voneinander entfernt sind.

Ergänzend kann die Proportionalitätsannahme etwa durch Einbeziehen von Interaktionstermen der einzelnen Variablen mit der Zeit überprüft werden; siehe 


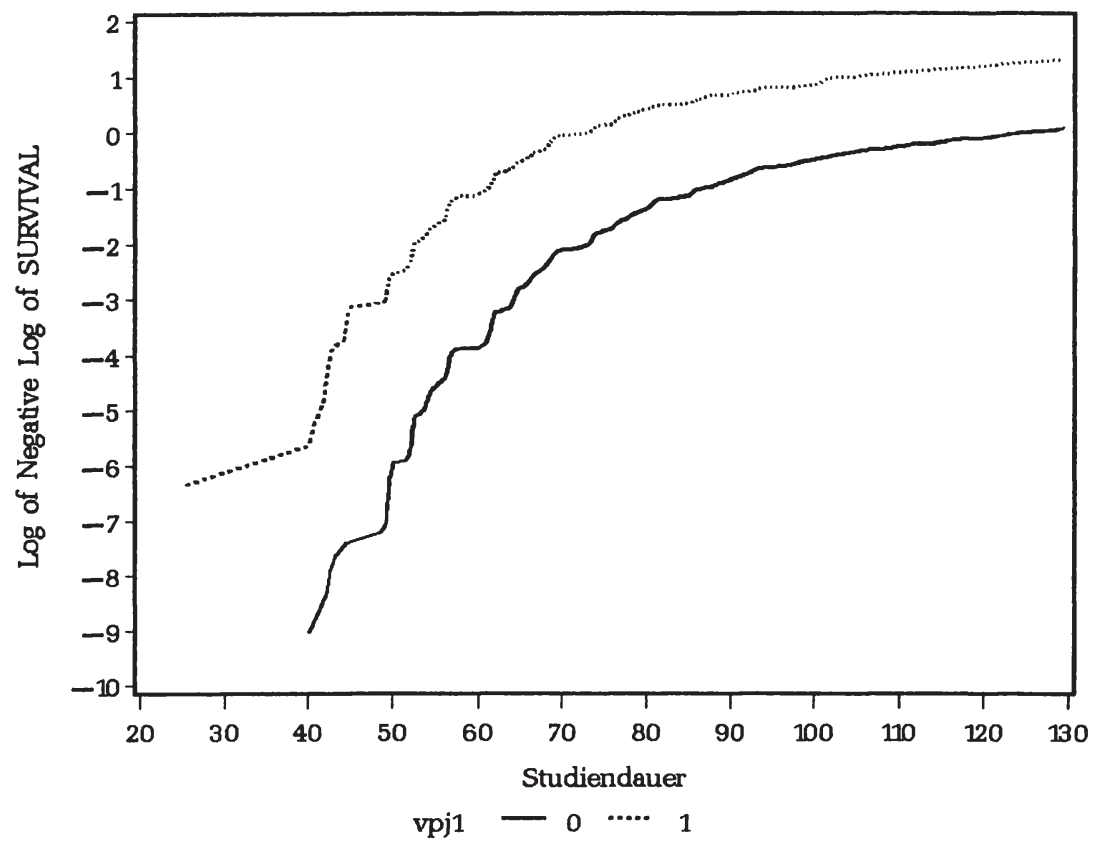

Abbildung 12.10: Plot der geschichteten (Kaplan-Meier-) Überlebensfunktionen für die binäre Variable "vpj1" (Studiendauer in Monaten)

Abschnitt 6.5. Für den vorliegenden Datensatz wurden in Anlehnung an die Abbildungen 12.10, 12.11 und 12.12 die Interaktionvariablen "vpj1time=vpj1 $\times I_{t \leq 72}$ ", "posj1time=gesposj $1 \times I_{t \leq 72}$ " und "avtime=average $\times I_{t \leq 72}$ ", sowie ergänzend Interaktionen der Variablen aus Tabelle 12.8 mit $\ln t, t \geq 42$, gebildet. Während die Interaktionsterme mit den Prüfungsvariablen $p$-Werte kleiner als 0.0001 liefern, zeigen alle anderen gebildeten Interaktionsvariablen mit der Zeit keinen Einfluss auf die (logarithmierte) Hazardrate $(p>0.1)$; auch graphische Überprüfungen zeigen für diese Variablen keine offensichtlichen Verletzungen der Proportionalitätsannahme. Daher wird angenommen, dass abgesehen von den Leistungsvariablen die einbezogenen Kovariablen die Proportionalitätsannahme erfüllen. Tabelle 12.9 zeigt die Parameterschätzer des finalen Modells nach Implementierung der Leistungsvariablen und der Interaktionsterme "vpj1time", "posj1time" und "avtime".

Die Regressionskoeffizienten des (finalen) Modells aus Tabelle 12.9 sind (auszugsweise) wie folgt zu interpretieren: 


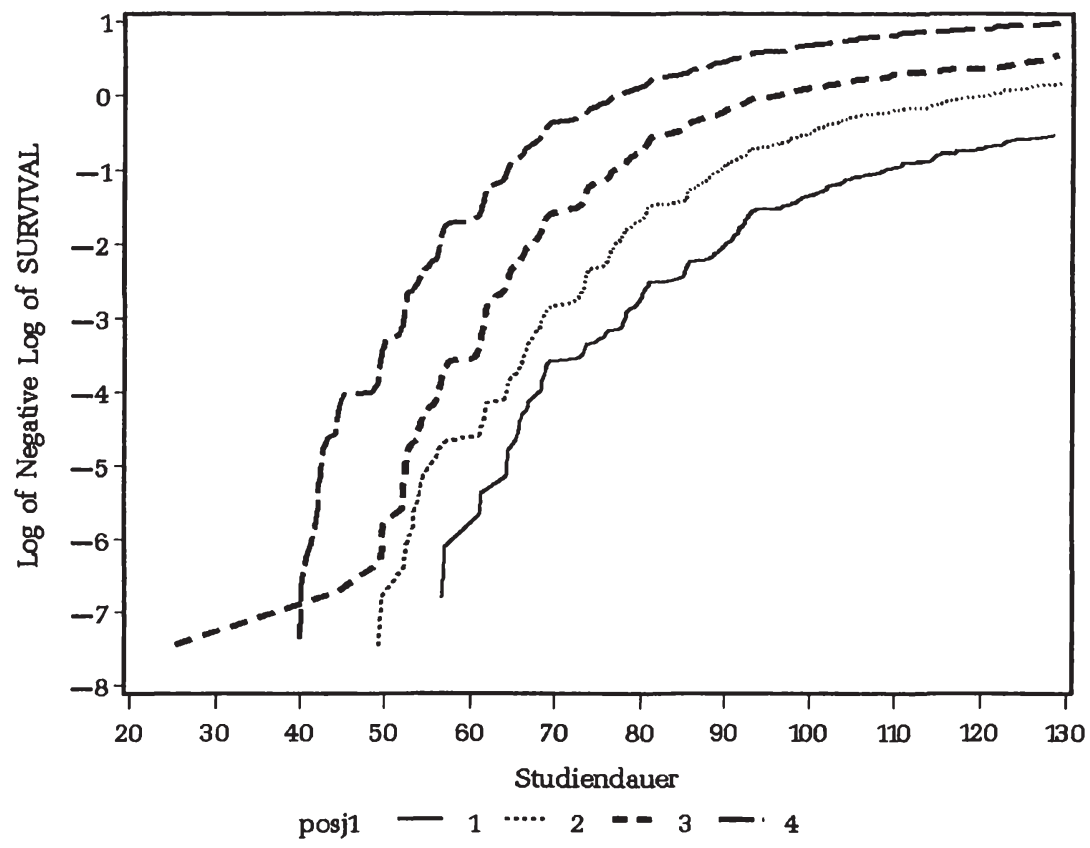

Abbildung 12.11: Plot der geschichteten (Kaplan-Meier-) Überlebensfunktionen für die gruppierte Variable "posj1"

- "matalt1": Studierende, die bei der Matura älter als 20 Jahre sind, haben zu jedem Zeitpunkt $t(\geq 42)$ bei gleichen Ausprägungen der sonstigen Variablen eine um $18 \%$ geringere Hazardrate für den Studienabschluss als die Referenzkategorie; $p=0.0096$. Die Variable "matalt1" liefert somit auch nach der Einbeziehung der Leistungsvariablen eine beinahe unveränderte Interpretation.

- "hak, htl": Analog zur Analyse der Reststudiendauer mit Hilfe des loglogistischen Modells verändert die Variable "hak" nach Einbeziehung der Prüfungsdaten ihre Richtung; ein HAK-Abschluss führt bei gleichen erbrachten Leistungen im ersten Studienjahr zu einer geringeren Hazardrate gegenüber AHS-AbsolventInnen.

Ein HTL-Abschluss verringert (bei gleicher Ausprägung aller anderen einbezogenen Variablen) die Studienabschlussrate.

- "gesposj1" und "posj1time": Je höher die Anzahl der positiven Leistungen im ersten Studienjahr, desto höher ist zu jedem Zeitpunkt die Chance auf den Studienabschluss. Dieser Effekt ist besonders ausgeprägt für die Zeit bis 


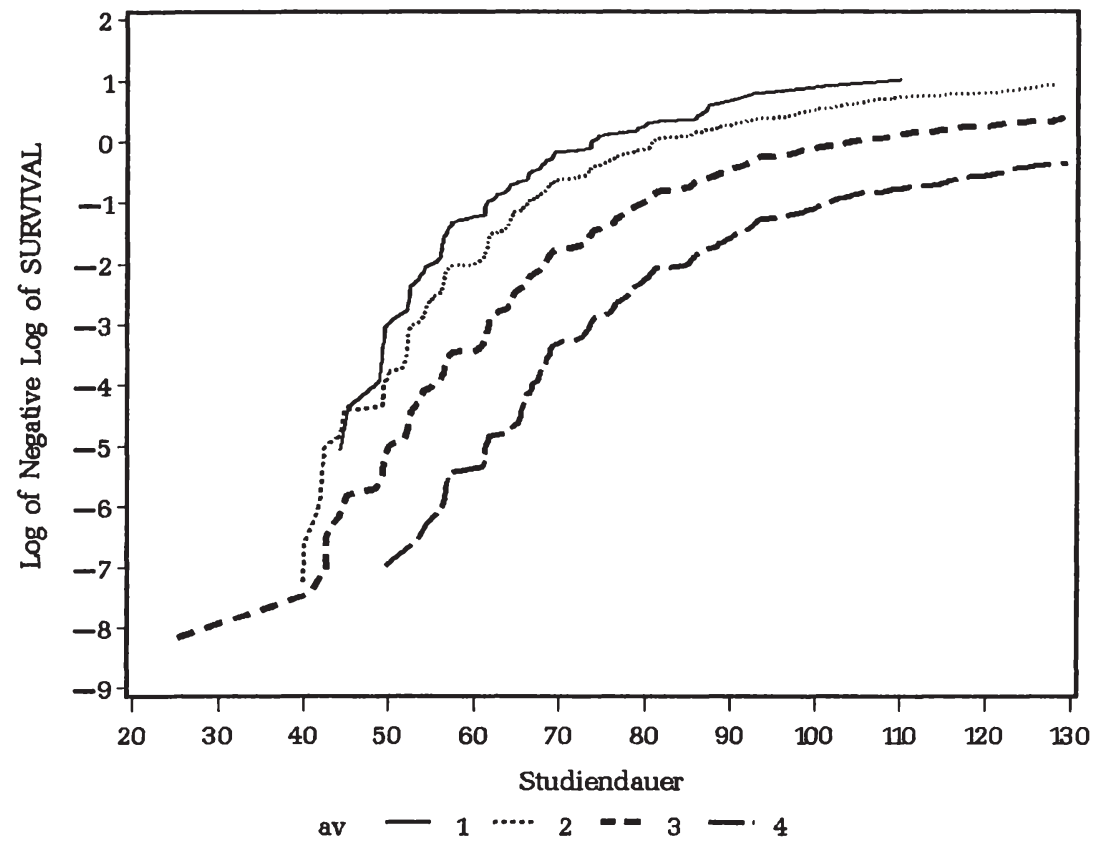

Abbildung 12.12: Plot der geschichteten (Kaplan-Meier-) Überlebensfunktionen für die gruppierte Variable "av"

zum Ende des zwölften Semesters (exp $(0.1+0.1)-1=22 \%$ pro Prüfung). Für die Zeit danach lässt der Effekt der positiven Leistungen des ersten Studienjahrs nach (11\%).

- "vpj1" und "vpj1time": Das Ablegen mindestens einer positiven Vor- bzw. Teildiplomprüfung (im ersten Studienjahr) erhöht die Chance auf den Studienabschluss. Dieser Effekt ist besonders ausgeprägt für die Zeit bis zum Ende des zwölften Semesters $(\exp (0.21+0.50)-1=103 \%)$. Für die Zeit danach lässt der Effekt nach (24\%).

- "average" und "avtime": Ein um ein Grad schlechterer (besserer) Notendurchschnitt erhöht (verringert) die Studienabschlussrate - um knapp $40 \%$ für Studienabschlüsse bis zum zwölften Semester und um knapp $10 \%$ für spätere Abschlüsse.

Werden bei vorhandenen zeitvariierenden Effekten von Variablen nicht die entsprechenden Interaktionsvariablen in das Modell einbezogen, so können die Parameterschätzer dieser Variablen als durchschnittlicher Effekt (über die Zeit) 
Tabelle 12.9: Parameterschätzer eines semiparametrischen Cox-Modells für den Studienabbschluss auf Basis der Stamm- und Prüfungsdaten (Jahrgänge 1989 bis 1993)

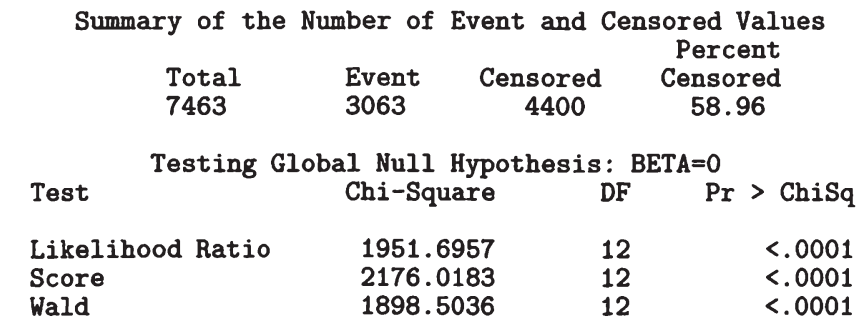

Analysis of Maximum Likelihood Estimates

$\begin{array}{lrrrrrr}\text { Variable } & \text { DF } & \begin{array}{r}\text { Parameter } \\ \text { Estimate }\end{array} & \begin{array}{r}\text { Standard } \\ \text { Error }\end{array} & \text { Chi-Square } & \text { Pr }>\text { ChiSq } & \begin{array}{r}\text { Hazard } \\ \text { Ratio }\end{array} \\ & & & & & & \\ \text { matalt1 } & 1 & -0.19758 & 0.07632 & 6.7022 & 0.0096 & 0.821 * * * \\ \text { lag1 } & 1 & -0.36983 & 0.12768 & 8.3905 & 0.0038 & 0.691 * * * \\ \text { sex } & 1 & -0.16187 & 0.05440 & 8.8530 & 0.0029 & 0.851 * * * \\ \text { sex*ahs } & 1 & -0.17570 & 0.07100 & 6.1244 & 0.0133 & 0.839 * * \\ \text { hak } & 1 & -0.28302 & 0.05162 & 30.0578 & <.0001 & 0.754 * * * \\ \text { ht1 } & 1 & -0.22269 & 0.07435 & 8.9708 & 0.0027 & 0.800 * * * \\ \text { gesposj1 } & 1 & 0.10370 & 0.00882 & 138.2860 & <.0001 & 1.109 * * * \\ \text { posj1time } & 1 & 0.10325 & 0.01448 & 50.8710 & <.0001 & 1.109 * * * \\ \text { vpj1 } & 1 & 0.21307 & 0.09713 & 4.8119 & 0.0283 & 1.237 * * * \\ \text { vpj1time } & 1 & 0.49884 & 0.12202 & 16.7142 & <.0001 & 1.647 * * * \\ \text { average } & 1 & -0.22396 & 0.04502 & 24.7467 & <.0001 & 0.799 * * * \\ \text { avtime } & 1 & -0.29010 & 0.07274 & 15.9046 & <.0001 & 0.748 * * * \\ \text { - } & -1 & & & & \end{array}$

auf die Studienabschlussrate gesehen werden. Das Hazard-Verhältnis der Variablen "gesposj1" etwa kann ohne Berücksichtigung der Interaktionsvariablen auf $14 \%$ geschätzt werden. Die anderen einbezogenen Variablen bleiben praktisch unverändert.

\section{Studienabschlussrate: Effekte in Abhängigkeit von Daten der Stichproben-Population}

In diesem Unterabschnitt werden die aus einer Befragung erhaltenen Daten für die (inländischen) Immatrikulierten der Wintersemester 1990 und 1993 (siehe Abschnitt 10.2) ergänzend zu den Daten aus der Hörerevidenz in das semiparametrische Cox-Modell für den Studienabschluss aufgenommen. Es gilt 
zu überprüfen, ob die Variablen

- Schul-Abschlussnoten aus Mathematik und Englisch

- Berufstätigkeit neben dem Studium

- zweite Ausbildung neben dem Studium

- Kinderbetreuung während des Studiums

die Hazardrate für den Studienabschluss beeinflussen. Der Schwerpunkt liegt in der Modellierung der Merkmale Berufstätigkeit, zweite Ausbildung und Kinderbetreuung als zeitabhängige Variablen.

Im Abschnitt 11.1 wurden die Merkmal-Verteilungen der Stichproben-Population mit den entsprechenden Verteilungen der gesamten Population verglichen. Es konnten keine überzufälligen Abweichungen der Stichprobe festgestellt werden. In einem zusätzlichen Vergleichsschritt werden nun die Ergebnisse der Implementierung eines semiparametrischen Cox-Modells für die Stammdaten aus der Hörerevidenz von (i) allen Immatrikulierten der Jahrgänge 1990 und 1993 (siehe Tabelle 12.10 für das entsprechende Modell nach Reduzierung von Variablen-Kategorien) und von (ii) den Befragten (siehe Tabelle 12.11) gegenübergestellt.

Die Parameterschätzer in den beiden Tabellen sind sehr ähnlich. Bildet man etwa in Tabelle 12.11 90\%-Konfidenzintervalle für die wichtigsten Parameterschätzer $(p<0.1)$, so fallen die entsprechenden Parameterschätzer aus Tabelle 12.10 in dieses Konfidenzintervall. Die Hypothese, dass alle Parameterschätzer gleich Null sind, wird in Tabelle 12.11 für alle drei Tests (LikelihoodQuotienten-, Score und Wald-Test) mit $p$-Werten kleiner als 0.001 abgelehnt; allerdings haben (im Gegensatz zu Tabelle 12.10) nur drei Variablen ("sex*ahs", "matalt1" und "woche5") einen $p$-Wert kleiner als 0.1. Dies ist auf das weitaus kleinere $n=703$ (gegenüber $n=3572$ ) zurückzuführen. Es sei noch angemerkt, dass die Analyse aus Tabelle 12.10 ähnliche Ergebnisse liefert wie die entsprechende Analyse für die Jahrgänge 1989 bis 1993 (vgl. Tabelle 12.8).

Im nächsten Schritt wurden zusätzlich zu den Variablen aus der Hörerevidenz die aus der Befragung gewonnene Information über die Schul-Abschlussnoten aus Mathematik und Englisch sowie über die Berufstätigkeit im ersten Semester (gemessen in durchschnittlichen Wochenstunden) in das Modell einbezogen. Dieses Modell setzt sich somit mit der Frage auseinander, welche der einbezogenen, zu Beginn des Studiums (bzw. nach einem Semester) bekannten demographischen Merkmale einen Einfluss auf die Studiendauer haben. 
Tabelle 12.10: Parameterschätzer eines semiparametrischen Cox-Modells für den Studienabschluss für die Jahrgänge 1990 und 1993

\begin{tabular}{|c|c|c|c|c|c|c|}
\hline \multicolumn{7}{|c|}{$\begin{array}{c}\text { Summary of the Number of Event and Censored Values } \\
\text { Percent }\end{array}$} \\
\hline \multicolumn{3}{|r|}{3572} & 1162 & 2410 & \multicolumn{2}{|l|}{67.47} \\
\hline \multicolumn{7}{|c|}{ Testing Global Null Hypothesis: BETA=0 } \\
\hline \multicolumn{3}{|l|}{ Test } & Chi-Square & $\mathrm{DF}$ & \multicolumn{2}{|c|}{$\operatorname{Pr}>\mathrm{ChiSq}$} \\
\hline \multicolumn{3}{|c|}{$\begin{array}{l}\text { Likelihood Ratio } \\
\text { Score } \\
\text { Wald }\end{array}$} & $\begin{array}{l}131.3220 \\
113.8252 \\
107.9484\end{array}$ & $\begin{array}{l}11 \\
11 \\
11\end{array}$ & \multicolumn{2}{|c|}{$\begin{array}{l}<.0001 \\
<.0001 \\
<.0001\end{array}$} \\
\hline \multicolumn{7}{|c|}{ Analysis of Maximum Likelihood Estimates } \\
\hline Variable & DF & $\begin{array}{l}\text { Parameter } \\
\text { Estimate }\end{array}$ & $\begin{array}{l}\text { Standard } \\
\text { Error }\end{array}$ & Chi-Square Pr & $>$ ChiSq & $\begin{array}{r}\text { Hazard } \\
\text { Ratio }\end{array}$ \\
\hline $\begin{array}{l}\text { matalt1 } \\
\text { lag1 } \\
\text { lag34 } \\
\text { sex } \\
\text { sex*ahs } \\
\text { hak } \\
\text { sex*woche2 } \\
\text { woche2 } \\
\text { woche3 } \\
\text { woche4 } \\
\text { woche5 }\end{array}$ & $\begin{array}{l}1 \\
1 \\
1 \\
1 \\
1 \\
1 \\
1 \\
1 \\
1 \\
1 \\
1\end{array}$ & $\begin{array}{r}-0.54104 \\
-0.58358 \\
0.12173 \\
-0.15328 \\
-0.22112 \\
0.21002 \\
0.22059 \\
-0.27386 \\
-0.19230 \\
-0.26548 \\
-0.88519\end{array}$ & $\begin{array}{l}0.13311 \\
0.26158 \\
0.07321 \\
0.07986 \\
0.09304 \\
0.06878 \\
0.15301 \\
0.11653 \\
0.11649 \\
0.22354 \\
0.24480\end{array}$ & $\begin{array}{r}16.5212 \\
4.9773 \\
2.7646 \\
3.6839 \\
5.6482 \\
9.3238 \\
2.0784 \\
5.5231 \\
2.7249 \\
1.4104 \\
13.0750\end{array}$ & $\begin{array}{l}<.0001 \\
0.0257 \\
0.0964 \\
0.0549 \\
0.0175 \\
0.0023 \\
0.1494 \\
0.0188 \\
0.0988 \\
0.2350 \\
0.0003\end{array}$ & $\begin{array}{l}0.582 * * * \\
0.558 * * \\
1.129 * \\
0.858 * \\
0.802 * * \\
1.234 * * * \\
1.247 \\
0.760 * * \\
0.825 * \\
0.767 \\
0.413 * * *\end{array}$ \\
\hline
\end{tabular}

Beide die Noten betreffenden kategoriellen Variablen wurden (aufgrund von vorangegangenen Modellüberprüfungen) als binäre Variablen ("mathe34" bzw. "englisch34") in das Modell aufgenommen, die die Noten Eins und Zwei in die Referenzkategorie zusammenfassen. Die graphische Überprüfung lässt für beide Variablen leichte Verletzungen der Proportionalitätsannahme erkennen. Abbildung 12.13 zeigt für "englisch34", dass etwa bis zum Ende des dreizehnten Semester die Kurven (zumeist) etwas weiter auseinander liegen als danach. Dies lässt vermuten, dass in späteren Semestern der Einfluss der Schulnoten auf die Studienabschlussrate nachlässt. Neben den Haupteffekten wurden daher die Interaktionsvariablen "englischtime $=$ englisch $34 \times I_{t \leq 78}$ " und "mathetime $=$ mathe $34 \times I_{t \leq 78}$ " in das Cox-Modell implementiert.

Die kontinuierlich skalierte Variable Berufstätigkeit im ersten Semester wurde analog zu Abschnitt 11.1 in die vier Kategorien "nicht berufstätig" (Re- 
Tabelle 12.11: Parameterschätzer eines semiparametrischen Cox-Modells für den Studienabschluss auf Basis einer Stichprobe der Jahrgänge 1990 und 1993

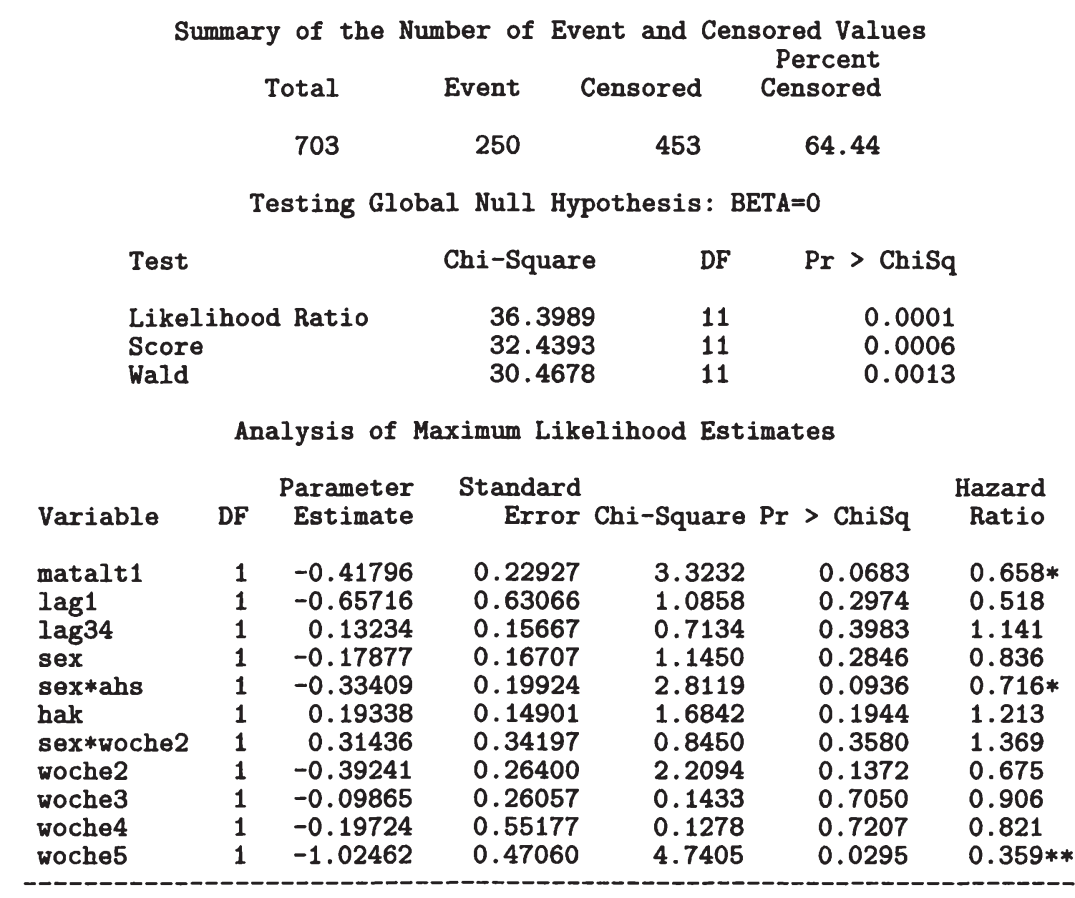

$* \mathrm{p}<0.1 ; * * \mathrm{p}<0.05 ; * * * \mathrm{p}<0.01$

ferenzkategorie), "geringfügig - (=job1)", "teilzeit - (=job2)" und "vollzeit beschäftigt (=job3)" unterteilt. Bei Implementierung dieses Modells erhalten wir die Parameterschätzer in Tabelle 12.12, wobei die Tabelle nur noch jene Variablen aus der Hörerevidenz mit $p \leq 0.3$ (und die entsprechenden Haupteffekte) zeigt.

Die Parameterschätzer der Variablen aus der Hörerevidenz zeigen sich nach Einbeziehung der ergänzenden Variablen aus der Befragung leicht verändert gegenüber Tabelle 12.11. Die Richtung der Variablen blieb gleich, jedoch änderte sich bei einigen Variablen der $p$-Wert. Die Parameterschätzer der aus der Befragung gewonnenen Variablen zeigen größtenteils einen hohen Erklärungsbeitrag (geringe $p$-Werte) und sind wie folgt zu interpretieren:

- "mathe34, mathetime, englisch34, englischtime": Die Haupteffekte "ma- 


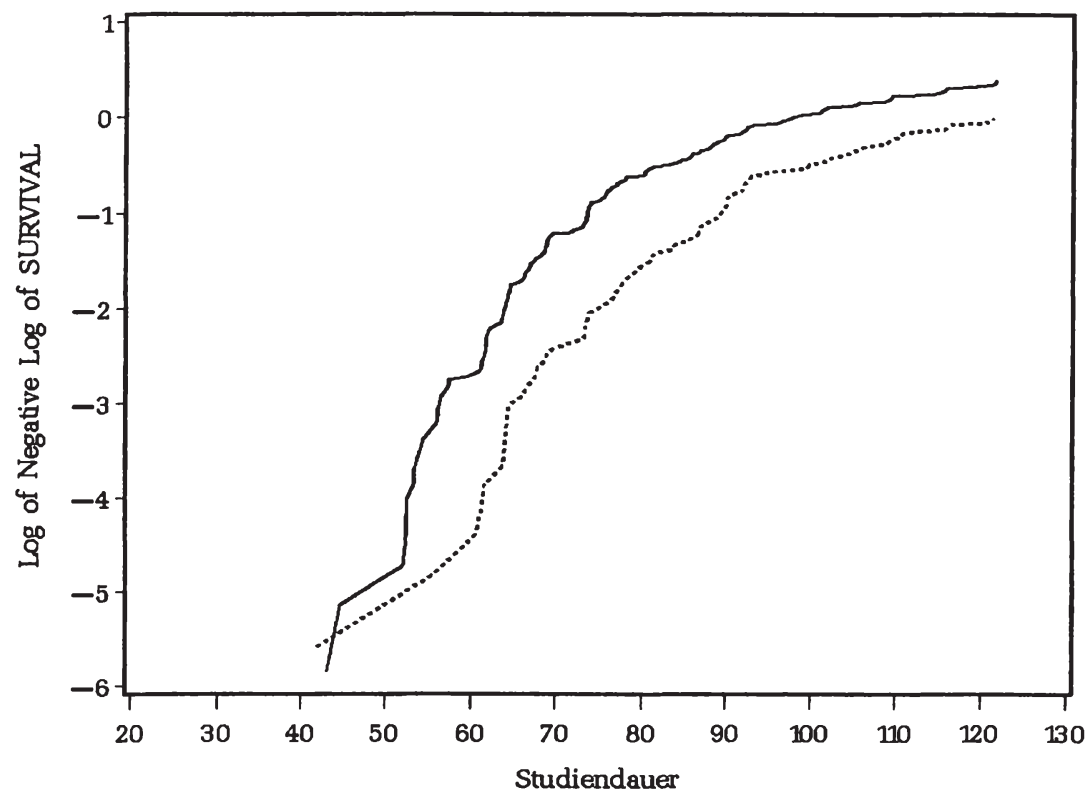

englisch34 $-0 \quad \cdots \cdot 1$

Abbildung 12.13: Plot der geschichteten (Kaplan-Meier-)Überlebensfunktionen für die binäre Variable "englisch34"

the34" und "englisch34", die sich auf Studienabschlüsse nach dem dreizehnten Semester beziehen, zeigen keinen (überzufälligen) Einfluss auf die Studienabschlussrate. Bei Studienabschlüssen vor dem dreizehnten Semester hingegen zeigen die Schulnoten den erwarteten negativen Effekt auf die Studienabschlussrate. Die Hazardrate für den Studienabschluss ist bis zum dreizehnten Semester für Studienanfänger, die die Schule in Mathematik bzw. in Englisch mit den Noten Drei oder Vier abgeschlossen haben, unter gleicher Ausprägung der restlichen einbezogenen Variablen (durchschnittlich) um $\exp (-0.95-0.18)=68 \%$ bzw. $51 \%$ geringer als von Immatrikulierten mit den Noten Eins oder Zwei in diesen Fächern. Bezieht man keine Interaktionsvariablen mit der Zeit ein, so können die entsprechenden Regressionskoeffizienten als durchschnittliche Effekte über die Zeit interpretiert werden.

- Beschäftigung im ersten Semester: Etwas überraschend ist der positive Koeffizient $(p=0.02)$ der Variablen "geringfügig beschäftigt" ("job1"), der besagt, dass die (im ersten Semester) geringfügig Beschäftigten eine (um 
Tabelle 12.12: Parameterschätzer eines semiparametrischen Cox-Modells für den Studienabschluss unter ergänzender Berücksichtigung von zeitunabhängigen Variablen aus der Befragung

Testing Global Null Hypothesis: BETA=0

$\begin{array}{lrrr}\text { Test } & \text { Chi-Square } & \text { DF } & \text { Pr }>\text { ChiSq } \\ \text { Likelihood Ratio } & 115.4712 & 13 & <.0001 \\ \text { Score } & 104.8895 & 13 & <.0001 \\ \text { Wald } & 94.0880 & 13 & <.0001\end{array}$

Analysis of Maximum Likelihood Estimates

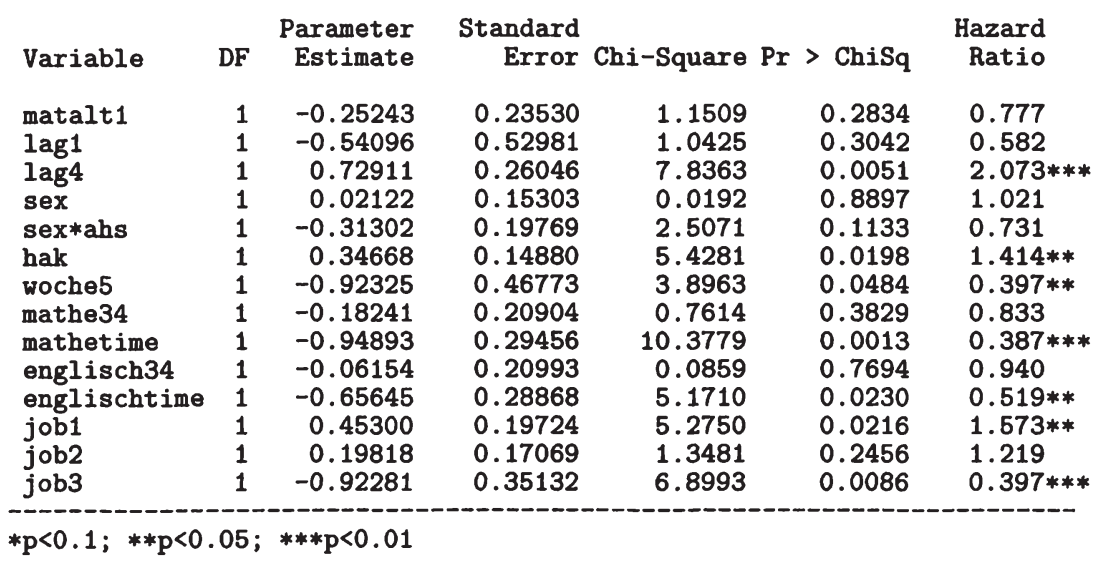

$57 \%$ ) höhere Hazardrate für den Studienabschluss als Nicht-Beschäftigte haben. Vollzeit Berufstätige weisen dagegen - wie erwartet - eine deutlich niedrigere Studienabschlussrate auf. Mehr Aufschluss über den Einfluss der Berufstätigkeit gibt die Modellierung der Variablen als zeitabhängige Größe.

Als Alternative wurden die letzten beiden Modelle auf Basis der in Abschnitt 10.2 angeführten Definition (c) - die Studiendauer ist die Zeit zwischen Studienbeginn und dem auf Befragung angegebenen Datum des Studienabschlusses bzw. Studienabbruchs - für die Studiendauer implementiert; es konnten keine überzufälligen Unterschiede in den Ergebnissen beobachtet werden.

Im letzten Schritt wurden dann die erhobenen zeitabhängigen Variablen in das Cox-Modell einbezogen. Mit diesen Variablen wird der Frage nachgegangen, ob

- der Beginn einer Erwerbstätigkeit bzw. eine Steigerung der Berufsintensität, oder 
- der Beginn bzw. das (ernsthafte) Betreiben eines zweiten Universitätsstudiums, oder

- die Geburt und folglich die Betreuung eines Kindes

den Studienverlauf verzögern.

Wie im Abschnitt 6.3 diskutiert, können zeitveränderliche Variablen je nach Fragestellung unterschiedlich in ein Modell aufgenommen werden. In einem ersten Schritt muss das unterschiedliche Messniveau der abhängigen Variablen (Studiendauer in Monaten) und der zeitveränderlichen Kovariablen (Berufsintensität je Semester) berücksichtigt werden. Dazu werden die Werte der zeitabhängigen Kovariablen zum Zeitpunkt (Monat) eines Ereignisses durch die Werte der Kovariablen in jenem Semester, in dem das Ereignis eingetroffen ist, approximiert. Danach wurde die Berufstätigkeit in verschiedenen Varianten in das Modell aufgenommen:

- als Berufsintensität im Vorsemester eines Studienabschlusses; in diesem Fall wird zu jedem Zeitpunkt eines Studienabschlusses für alle Studierenden aus der Risikomenge die Berufsintensität des Vorsemesters ermittelt und verglichen.

- als durchschnittliches Berufsausmaß in den letzten drei Semestern vor einem Studienabschluss; für jeden Studierenden aus der Risikomenge wird zu jedem Zeitpunkt eines Ereignisses die durchschnittliche Berufsintensität in den drei Semestern zuvor ermittelt.

- als durchschnittliches Berufsausmaß seit Studienbeginn; für jeden Studierenden aus der Risikomenge wird zu jedem Zeitpunkt eines Ereignisses die durchschnittliche Berufsintensität seit Studienbeginn ermittelt.

Bei allen drei Varianten wird das durchschnittliche Berufsausmaß im Semester des Studienabschlusses nicht berücksichtigt, da die Möglichkeit besteht, dass Studierende zu Beginn eines Semesters abgeschlossen haben und in Folge die Berufsintensität (in diesem Semester) erhöht haben (wechselseitige Beeinflussung); siehe Abschnitt 6.3. Bei der getrennten Implementierung dieser Variablen zeigt sich, dass jede Variable für sich einen massiven Einfluss auf die Studienabschlussrate hat. Werden die Variablen gemeinsam in das Modell aufgenommen, so ist zu erkennen, dass die drei Variablen - wie vermutet - stark korrelieren. In das finale Modell wurde die Berufstätigkeit im Vorsemester ("emp") und die durchschnittliche Berufsintensität seit Studienbeginn ("empav") als kontinuierlich skalierte Variablen aufgenommen. 
Auch die zeitabhängige Variable, die die zweite Ausbildung parallel zum WUStudium betrifft, kann in verschiedenen Varianten in das Modell aufgenommen werden:

- als binäre Variable, die zu jedem Zeitpunkt eines Studienabschlusses für alle Studierenden aus der Risikomenge angibt, ob im laufenden Semester (oder im Vorsemester, keine Unterschiede im Ergebnis) eine zweite Ausbildung besucht wird oder nicht;

- als kontinuierliche Variable, die zu jedem Zeitpunkt eines Studienabschlusses für alle Studierenden aus der Risikomenge angibt, wieviele Semester sie bisher in eine zweite Ausbildung neben dem WU-Studium investiert haben.

Bei getrennter Implementierung dieser beiden Variablen zeigt die Anzahl der Semester in einer zweiten Ausbildung einen kleineren $p$-Wert und wird daher in das finale Modell aufgenommen ("cumbildg").

Die Variable Kinderbetreuung kann analog zur zweiten Ausbildung implementiert werden. Auch in diesem Fall wird die Anzahl der Semester mit Kinderbetreuung als zeitveränderliche Variable in das Modell aufgenommen ("cumkind").

Durch Aufnahme der eben erläuterten zeitveränderlichen Variablen in das Modell erhalten wir die Tabelle 12.13. Die Berücksichtigung der zeitabhängigen Variablen erfordert die Implementierung der Studiendauer (bis zum Studienabschluss bzw. bis zum Studienabbruch) entsprechend Definition (c), da diese Variablen auch nur bis zum selbst genannten Semester des Studienabschlusses bzw. Studienabbruchs beobachtet werden konnten.

Alle zeitveränderlichen Variablen zeigen einen deutlichen Effekt auf die Studienabschlussrate. Die Richtungen der Parameterschätzer der zeitveränderlichen Variablen sind alle wie erwartet. Je mehr

- Wochenstunden im Semester zuvor (und auch durchschnittlich) in einen Job,

- Semester für eine zweite Ausbildung und für Kinderbetreuung

investiert wurden, desto geringer ist (zu jedem Zeitpunkt $t(\geq 42)$ ) die Studienabschlussrate. Am Hazard-Verhältnis kann wieder die Größenordnung dieser Unterschiede abgelesen werden. Jedes Semester mehr, das in eine zweite Ausbildung seit Studienbeginn investiert wurde, verringert die Studienabschlussrate um knapp 10\%. Ergänzend wurde noch getestet, ob die Variable "cumkind" 
Tabelle 12.13: Parameterschätzer eines semiparametrischen Cox-Modells für den Studienabschluss unter Berücksichtigung der erhobenen zeitabhängigen Variablen

Testing Global Null Hypothesis: BETA=0

$\begin{array}{lrrr}\text { Test } & \text { Chi-Square } & \text { DF } & \text { Pr }>\text { ChiSq } \\ \text { Likelihood Ratio } & 147.0680 & 13 & <.0001 \\ \text { Score } & 132.0959 & 13 & <.0001 \\ \text { Wald } & 128.1594 & 13 & <.0001\end{array}$

Analysis of Maximum Likelihood Estimates

$\begin{array}{lrrrrrl}\text { Variable } & \text { DF } & \begin{array}{r}\text { Parameter } \\ \text { Estimate }\end{array} & \begin{array}{r}\text { Standard } \\ \text { Error }\end{array} & \text { Chi-Square } & \text { Pr }>\text { ChiSq } & \begin{array}{c}\text { Hazard } \\ \text { Ratio }\end{array} \\ \text { matalt1 } & 1 & -0.39383 & 0.23095 & 2.9080 & 0.0881 & 0.674 * \\ \text { lag1 } & 1 & -0.78372 & 0.52435 & 2.2340 & 0.1350 & 0.457 \\ \text { lag4 } & 1 & 0.62806 & 0.26554 & 5.5944 & 0.0180 & 1.874 * * \\ \text { sex } & 1 & 0.30303 & 0.16994 & 3.1797 & 0.0746 & 1.354 * \\ \text { sex*ahs } & 1 & -0.68226 & 0.20017 & 11.6174 & 0.0007 & 0.505 * * * \\ \text { ht1 } & 1 & -0.38383 & 0.21727 & 3.1209 & 0.0773 & 0.681 * \\ \text { woche5 } & 1 & -0.58984 & 0.47186 & 1.5626 & 0.2113 & 0.554 \\ \text { mathe34 } & 1 & -0.56918 & 0.14262 & 15.9281 & <.0001 & 0.566 * * * \\ \text { englisch34 } & 1 & -0.36884 & 0.14400 & 6.5610 & 0.0104 & 0.692 * * \\ \text { empav } & 1 & -0.02067 & 0.00975 & 4.4892 & 0.0341 & 0.980 * * \\ \text { emp } & 1 & -0.02327 & 0.00693 & 11.2645 & 0.0008 & 0.977 * * \\ \text { cumbildg } & 1 & -0.10440 & 0.03440 & 9.2114 & 0.0024 & 0.901 * * * \\ \text { cumkind } & 1 & -0.11768 & 0.05282 & 4.9639 & 0.0259 & 0.889 * *\end{array}$

$* \mathrm{p}<0.1 ; * * \mathrm{p}<0.05 ; * * * \mathrm{p}<0.01$

Wechselwirkungen mit dem Geschlecht zeigt. Die Ergebnisse dieses Modells zeigen (wie erwartet) eine Verstärkung des negativen Effekts auf die Studienabschlussrate für weibliche und eine Abschwächung für männliche Studierende. Aufgrund der geringen Anzahl von Studierenden (siehe Tabelle 11.21), die angeben, mindestens ein Semester ein Kind betreut zu haben, und der noch geringeren $\mathrm{Zahl}$ von Absolventen unter diesen Studierenden sind die Ergebnisse allerdings mit Vorsicht $\mathrm{zu}$ interpretieren.

Die Variablen der Hörerevidenz behalten mehrheitlich auch nach Einbeziehen der zeitabhängigen Variablen ihre Interpretation - etwa die verringernde Wirkung der Variablen "matalt1" und "woche5" auf die Studienabschlussrate.

Es sei noch abschließend bemerkt, dass auch nach Einbeziehen der prädiktiven Prüfungsdaten aus dem ersten Studienjahr die Variablen "emp" (bzw. "empav" bei getrennter Implementierung) und "cumkind" unverändert einen verringernden Einfluss $(p<0.01)$ auf die Studienabschlussrate haben. 


\section{Kapitel 13}

\section{Analyse des Studienabbruchs}

\subsection{Einleitung}

Ziel der Analysen diese Kapitels ist die Ermittlung von Variablen, die das Risiko eines Studienabbruchs erhöhen, wobei vermutet wird, dass die Erklärungsfaktoren je nach Zeitpunkt des Studienabbruchs unterschiedlich sein können. Insbesondere der Studienabbruch in der Anfangsphase eines Studiums kann häufig ganz andere Gründe haben als der Studienabbruch in einer späteren Phase des Studiums. So ist zum Beispiel zu erwarten, dass der Zeitpunkt der Immatrikulation vor allem zu Studienbeginn einen starken Einfluss auf die Studienabbruchrate hat, der dann mit der Zeit abnimmt.

Aufgrund der deskriptiven Analysen aus Abschnitt 11.1 ist bekannt, dass ein hoher Anteil der Immatrikulierten das Studium abbrechen ohne eine einzige (positive) Leistung erbracht zu haben (siehe Tabelle 11.1). Da zu vermuten ist, dass

- gerade diese Studienabbrecher andere Abbruchgründe als Spätabbrecher (siehe auch Tabelle 11.8 in Abschnitt 11.1) haben bzw.

- ein Großteil dieser Gruppe nicht wirklich zu den Studierenden der Wirtschaftsuniversität gezählt werden kann ("Schnupperstudierende"), und

- da insbesondere für diese Studienabbrecher auch keine passende Studiendauer angegeben werden kann,

werden in diesem Kapitel 
- Studienabbrüche ohne eine einzige positive Leistung (kann als Proxy für Studienabbrüche im ersten Semester gesehen werden), bzw.

- Studienabbrüche, die nach erfolgreichem Ablegen von Prüfungen erfolgen,

in getrennten Modellen analysiert.

Zeitvariierende Effekte bei Studienabbrüchen, die nach erfolgreichem Ablegen von Prüfungen erfolgen, können dann über die Einbeziehung einer entsprechenden Interaktionsvariablen zwischen dem betrachteten Merkmal und der Zeit in einem semiparametrischen Cox-Modell berücksichtigt werden.

Die Analyse der Wahrscheinlichkeit eines Studienabbruchs ohne positive Leistung erfolgte mit Hilfe eines logistischen Regressionsmodells; für die Analyse des Studienabbruch-Risikos mit positiven Prüfungsergebnissen ist das übergangsspezifische semiparametrische Modell von Cox implementiert worden. Da die Analyse der Verteilung der Studiendauer bis zum Studienabbruch nicht im Mittelpunkt des Interesses steht, wird in dieser Arbeit nicht auf die Implementierung von log-linearen Regressionsmodellen für den Studienabbruch eingegangen.

Als Datenbasis wurden einerseits wieder die Daten der inländischen Immatrikulierten der Wintersemester 1989/90 bis 1993/94 (gesamte Population) und andererseits die Daten aus der ergänzenden Befragung (Stichproben-Population) herangezogen. Der Studienabbruch wurde für die Analyse der gesamten Population entsprechend Definition (B) aus Abschnitt 10.1 verstanden.

\subsection{Analyse der Studienabbruch-Wahrschein- lichkeit ohne positive Leistung}

\section{Modelle auf Basis der Stammdaten aus der Hörerevidenz}

Zur Analyse der Wahrscheinlichkeit des Studienabbruchs ohne positive Leistung wurde das logistische Regressionsmodell

$$
\pi(\mathbf{x})=\frac{\exp \left(\beta_{0}+\mathbf{x}^{\prime} \boldsymbol{\beta}\right)}{1+\exp \left(\beta_{0}+\mathbf{x}^{\prime} \boldsymbol{\beta}\right)}
$$

implementiert. Dabei ist $\pi(\mathbf{x})$ die Wahrscheinlichkeitsfunktion, dass das binäre Merkmal "Abbruch ohne positive Leistung" für ein Individuum als zutreffend 
bewertet wird. Das Modell wird häufig auch als

$$
\ln \frac{\pi(\mathbf{x})}{1-\pi(\mathbf{x})}=\beta_{0}+\mathbf{x}^{\prime} \boldsymbol{\beta}
$$

angeschrieben. Das Verhältnis $\pi /(1-\pi)$ wird odds genannt; siehe auch Kapitel 8. Für dieses Modell erhält man für die Jahrgänge 1989 bis 1993 (ohne Berücksichtigung von Jahrgangsspezifika) unter Einbeziehung der Stammdaten aus der Hörerevidenz die Tabelle 13.1.

Tabelle 13.1: Parameterschätzer des logistischen Regressionsmodells für den Studienabbruch ohne positives Prüfungsergebnis (Jahrgänge 1989 bis 1993)

Response Profile

$\begin{array}{rrr}\begin{array}{r}\text { Ordered } \\ \text { Value }\end{array} & \text { pi } & \begin{array}{r}\text { Total } \\ \text { Frequency }\end{array} \\ 1 & 0 & 2034 \\ 2 & 1 & 7288\end{array}$

Probability modeled is $\mathrm{p} i=0$.

Testing Global Null Hypothesis: BETA=0

$\begin{array}{lrrr}\text { Test } & \text { Chi-Square } & \text { DF } & \text { Pr }>\text { ChiSq } \\ \text { Likelihood Ratio } & 1765.4482 & 14 & <.0001 \\ \text { Score } & 1912.8409 & 14 & <.0001 \\ \text { Wald } & 1408.3359 & 14 & <.0001\end{array}$

Analysis of Maximum Likelihood Estimates

\begin{tabular}{|c|c|c|c|c|c|}
\hline Parameter & DF & Estimate & $\begin{array}{r}\text { Standard } \\
\text { Error }\end{array}$ & $\begin{array}{r}\text { Wald } \\
\text { Chi-Square }\end{array}$ & $\mathrm{Pr}>\mathrm{ChiSq}$ \\
\hline Intercept & 1 & -2.0628 & 0.0625 & 1090.4237 & $<.0001$ \\
\hline matalt 1 & 1 & 0.8748 & 0.0750 & 136.1105 & $<.0001 * * *$ \\
\hline $\operatorname{lag} 1$ & 1 & -1.3370 & 0.1331 & 100.9497 & $<.0001 * * *$ \\
\hline $\operatorname{lag} 2$ & 1 & 0.4078 & 0.1612 & 6.3960 & $0.0114 * *$ \\
\hline lag3 & 1 & -0.0673 & 0.0893 & 0.5671 & 0.4514 \\
\hline $\operatorname{lag} 4$ & 1 & 0.8533 & 0.0807 & 111.7417 & $<.0001 * * *$ \\
\hline sex & 1 & -0.5657 & 0.0623 & 82.3562 & $<.0001 * * *$ \\
\hline hak & 1 & -0.2548 & 0.0722 & 12.4468 & $0.0004 * * *$ \\
\hline htl & 1 & 0.3682 & 0.0810 & 20.6786 & $<.0001 * * *$ \\
\hline hla & 1 & 0.2163 & 0.1258 & 2.9553 & $0.0856 *$ \\
\hline other & 1 & -0.0174 & 0.1525 & 0.0130 & 0.9093 \\
\hline woche2 & 1 & 0.7982 & 0.0730 & 119.5306 & $<.0001 * * *$ \\
\hline woche 3 & 1 & 1.2845 & 0.0875 & 215.4019 & $<.0001 * * *$ \\
\hline woche4 & 1 & 1.9569 & 0.1117 & 307.1054 & $<.0001 * * *$ \\
\hline woche 5 & 1 & 2.7318 & 0.1021 & 715.4547 & $<.0001 * * *$ \\
\hline
\end{tabular}

$* p<0.1 ; * * p<0.05 ; * * * p<0.01$ 
Die kontinuierlichen Variablen Alter bei der Matura ("matalt") und Zeitabstand zwischen Matura und Studienbeginn ("lag") wurden wie im vorangegangenen Kapitel als kategorielle Variablen implementiert. Die Parameterschätzer der Tabelle können wie folgt interpretiert werden:

- "matalt1": Der Koeffizient der Variablen ist stark positiv mit einem p-Wert kleiner als 0.0001. Die odds für einen Abbruch ohne positive Leistung ist (unter gleicher Ausprägung aller anderen einbezogenen Variablen) für Immatrikulierte, die bei der Matura älter als 20 Jahre sind, um $139 \%$ höher als für Studierende der Referenzkategorie.

- "lag1" bis "lag4": Zu beachten ist der negative Koeffizient von "lag1", der (unter gleicher Ausprägung der anderen einbezogenen Kovariablen) besagt, dass Studierende, die im Herbst des Studienbeginns maturiert haben, eine geringere Abbruchwahrscheinlichkeit ohne Leistung haben als Immatrikulierte, die zum Standard-Termin die Matura absolviert haben. Allerdings haben - wie erwähnt - fast alle Studierenden dieser Gruppe in der vierten oder fünften Woche immatrikuliert, und die Regressionskoeffizienten dieser beiden Variablen sind positiv und vom Betrag deutlich größer als der Koeffizient von "lag1". Ohne Einbeziehung der Immatrikulationswochen ist der Koeffizient von "lag1" positiv (mit $p<0.0001$ ).

- "sex": Erwähnenswert ist weiters der negative Koeffizient der Variablen Geschlecht $(p<0.0001)$, der besagt, dass Studentinnen eher ohne eine einzige Leistung abbrechen als Studenten (unter gleicher Ausprägung aller anderen einbezogenen Kovariablen).

- "hak, htl, hla, other": Der HTL-Abschluss zeigt einen (positiven) Koeffizienten mit p-Wert kleiner als 0.0001; HTL-Absolventen haben somit zu Studienbeginn eine höhere Abbruchwahrscheinlichkeit als AHS-Maturanten. HAK-Absolventen hingegen zeigen eine geringere Abbruchwahrscheinlichkeit ohne positives Prüfungsergebnis.

- "woche2" bis "woche5" Die Koeffizienten der Immatrikulationswochen sind alle - wie erwartet - stark positiv. Umso später immatrikuliert wird, desto höher ist die Wahrscheinlichkeit, ohne eine einzige positive Leistung das Studium an der Wirtschaftsuniversität abzubrechen.

Zur Überprüfung von Unterschieden zwischen den Geschlechtern wurden wieder Interaktionsvariablen des Geschlechts mit den restlichen Variablen implementiert bzw. getrennte Analysen durchgeführt. Dabei konnten keine bedeutenden Differenzen zwischen den Geschlechtern festgestellt werden. Auch an- 
dere erwähnenswerte Wechselwirkungen zwischen den Variablen konnten nicht beobachtet werden.

Bei Einbeziehen von binären Variablen für die Jahrgänge zeigt der Jahrgang 1993 eine geringere Wahrscheinlichkeit für einen Studienabbruch ohne positives Prüfungsergebnis im Vergleich mit den anderen Jahrgängen $(p<0.0001)$. Die restlichen Variablen zeigen sich nach Einbeziehen der binären Variablen praktisch unverändert. Ein Vergleich zwischen den Jahrgängen zeigt für den Großteil der Variablen (wie "matalt1", "lag1" und "woche2-woche5") in der Interpretation kaum Unterschiede.

Als Alternative zur Modellierung des frühen Studienabbruchs auf Basis der Stammdaten aus der Hörerevidenz können Studienabbrüche, die

- mit maximal zwei erbrachten positiven Leistungen, oder

- in den ersten drei Semestern (entsprechend Definition (b) aus Abschnitt 10.1) erfolgt sind,

analysiert werden. Entsprechende Analysen zeigen kaum Unterschiede in der Interpretation der einbezogenen Variablen.

\section{Modelle auf Basis von Daten der Stichproben-Population}

Im ersten Schritt wurde für Vergleichszwecke auf Basis der Variablen aus der Hörerevidenz das gleiche logistische Modell auf die Stichprobe und auf die gesamte Population der Jahrgänge 1990 und 1993 angewandt. Die Ergebnisse stimmen in Richtung und Größenordnung (und damit in der Interpretation) recht gut mit den Parameterschätzern aus Tabelle 13.1 überein. Tabelle 13.2 zeigt die Ergebnisse für die Stichproben-Population.

Danach wurden zur Analyse der Wahrscheinlichkeit des Studienabbruchs ohne positive Leistung ergänzend die in der Befragung erhobenen Variablen Schulnoten aus Mathematik und Englisch sowie die Berufstätigkeit im ersten Semester in das logistische Regressionsmodell aufgenommen.

Nach schrittweiser Reduktion der Variablen aus der Hörerevidenz und nach Einbeziehen der ergänzenden Variablen aus der Befragung analog zum Abschnitt 12.3 erhält man die Tabelle 13.3.

Die Koeffizienten der Variablen aus der Hörerevidenz zeigen sich nach Einbeziehen der ergänzenden Variablen ziemlich unverändert. Die Schul-Abschlussnoten beeinflussen den Studienabbruch ohne positive Leistung nicht $(p>0.25$, wenn 
Tabelle 13.2: Parameterschätzer eines logistischen Regressionsmodells für den Studienabbruch ohne positive Leistung auf Basis der Stichproben-Population

Response Profile

$\begin{array}{rrr}\begin{array}{r}\text { Ordered } \\ \text { Value }\end{array} & \text { pi } & \begin{array}{r}\text { Total } \\ \text { Frequency }\end{array} \\ 1 & 0 & 115 \\ 2 & 1 & 588\end{array}$

Testing Global Null Hypothesis: BETA=0

$\begin{array}{lrrr}\text { Test } & \text { Chi-Square } & \text { DF } & \text { Pr }>\text { ChiSq } \\ \text { Likelihood Ratio } & 83.3701 & 13 & <.0001 \\ \text { Score } & 91.0970 & 13 & <.0001 \\ \text { Wald } & 73.5043 & 13 & <.0001\end{array}$

Analysis of Maximum Likelihood Estimates

$\begin{array}{lrrrrr}\text { Parameter } & \text { DF } & \text { Estimate } & \begin{array}{r}\text { Standard } \\ \text { Error }\end{array} & \begin{array}{r}\text { Wald } \\ \text { Chi-Square }\end{array} & \text { Pr > ChiSq } \\ \text { Intercept } & 1 & -2.5014 & 0.2540 & 96.9591 & <.0001 \\ \text { matalt1 } & 1 & 1.0354 & 0.2555 & 16.4231 & <.0001 * * * \\ \text { lag1 } & 1 & -0.9589 & 0.5307 & 3.2650 & 0.0708 * \\ \text { lag2 } & 1 & -0.5842 & 0.8262 & 0.4999 & 0.4795 \\ \text { lag4 } & 1 & 0.9270 & 0.3131 & 8.7658 & 0.0031 * * \\ \text { sex } & 1 & -0.3648 & 0.2458 & 2.2024 & 0.1378 \\ \text { hak } & 1 & -0.1235 & 0.2821 & 0.1917 & 0.6615 \\ \text { ht1 } & 1 & 0.2185 & 0.3011 & 0.5265 & 0.4681 \\ \text { hla } & 1 & 0.7280 & 0.4370 & 2.7759 & 0.0957 * \\ \text { other } & 1 & 0.2997 & 0.6440 & 0.2165 & 0.6417 \\ \text { woche2 } & 1 & 0.9207 & 0.2789 & 10.8992 & 0.0010 * * * \\ \text { woche3 } & 1 & 1.1903 & 0.3381 & 12.3912 & 0.0004 * * * \\ \text { woche4 } & 1 & 1.9058 & 0.4242 & 20.1793 & <.0001 * * * \\ \text { woche5 } & 1 & 1.5321 & 0.4256 & 12.9583 & 0.0003 * * * \\ \text {------- - - - - } & 1 & & & \end{array}$

auch die Richtung wie erwartet ist). Etwas überraschend sind die Richtungen der Kategorien "geringfügig und teilweise beschäftigt im ersten Semester", die angeben, dass Studierende aus diesen Kategorien eine geringere Wahrscheinlichkeit für den Studienabbruch ohne Leistung haben als Studierende, die im ersten Semester nicht berufstätig waren; hierbei ist jedoch zu beachten, dass die $p$-Werte beider Kategorien größer als 0.15 sind. Die Berufstätigkeit mit einem Wochenstundenausmaß von mindestens 30 Stunden erhöht hingegen die Wahrscheinlichkeit eines frühen Studienabbruchs $(p<0.01)$.

Der Studienabbruch ohne Leistung hängt meist mit Unschlüssigkeit, mangelnder Zielstrebigkeit, enttäuschten Erwartungen, dem Beginn des Studiums als 
Tabelle 13.3: Parameterschätzer eines logistischen Regressionsmodells für den Studienabbruch ohne positive Leistung unter Berücksichtigung von ergänzenden Variablen aus der Befragung

Testing Global Null Hypothesis: BETA=0

$\begin{array}{lrrr}\text { Test } & \text { Chi-Square } & \text { DF } & \text { Pr }>\text { ChiSq } \\ \text { Likelihood Ratio } & 98.4333 & 15 & <.0001 \\ \text { Score } & 105.0342 & 15 & <.0001 \\ \text { Wald } & 82.1300 & 15 & <.0001\end{array}$

Analysis of Maximum Likelihood Estimates

\begin{tabular}{|c|c|c|c|c|c|}
\hline Parameter & DF & Estimate & $\begin{array}{r}\text { Standard } \\
\text { Error }\end{array}$ & $\begin{array}{r}\text { Wald } \\
\text { Chi-Square }\end{array}$ & $\mathrm{Pr}>\mathrm{ChiSq}$ \\
\hline Intercept & 1 & -2.5546 & 0.2880 & 78.6942 & $<.0001$ \\
\hline matalt 1 & 1 & 0.9460 & 0.2582 & 13.4236 & $0.0002 * * *$ \\
\hline $\operatorname{lag} 12$ & 1 & -0.7352 & 0.4703 & 2.4436 & 0.1180 \\
\hline lag4 & 1 & 0.7736 & 0.3257 & 5.6414 & $0.0175 * *$ \\
\hline sex & 1 & -0.4135 & 0.2459 & 2.8276 & $0.0927 *$ \\
\hline hak & 1 & -0.3121 & 0.2711 & 1.3247 & 0.2498 \\
\hline hla & 1 & 0.8517 & 0.4333 & 3.8637 & $0.0493 * *$ \\
\hline woche 2 & 1 & 0.8265 & 0.2839 & 8.4783 & $0.0036 * * *$ \\
\hline woche3 & 1 & 1.1913 & 0.3411 & 12.2000 & $0.0005 * * *$ \\
\hline woche4 & 1 & 1.6332 & 0.4343 & 14.1441 & $0.0002 * * *$ \\
\hline woche5 & 1 & 1.2555 & 0.4401 & 8.1375 & $0.0043 * * *$ \\
\hline mathe34 & 1 & 0.2507 & 0.2342 & 1.1462 & 0.2843 \\
\hline englisch34 & 1 & 0.1574 & 0.2350 & 0.4487 & 0.5030 \\
\hline job1 & 1 & -0.3657 & 0.4696 & 0.6066 & 0.4361 \\
\hline job2 & 1 & -0.5240 & 0.3690 & 2.0173 & 0.1555 \\
\hline job3 & 1 & 0.8321 & 0.2858 & 8.4789 & $0.0036 * * *$ \\
\hline
\end{tabular}

$* \mathrm{p}<0.1 ; * * \mathrm{p}<0.05 ; * * * \mathrm{p}<0.01$

Überbrückung etc. zusammen - Variablen, die schwierig zu messen sind. Tabelle 11.6 aus Abschnitt 11.1 zeigt, dass etwa die Hälfte der Studienabbrecher ohne Leistung zu Studienbeginn nicht sicher waren, ob sie das Studium auch abschließen wollen. Der Immatrikulationsbeginn kann als Hilfsvariable für Unschlüssigkeit bzw. mangelnde Zielstrebigkeit interpretiert werden. Die negativen Koeffizienten der beiden Kategorien "geringfügig und teilweise beschäftigt im ersten Semester"(mit $p$-Werten größer als 0.15) lassen vermuten, dass die Belastung einer beruflichen Tätigkeit unter 30 Stunden zumeist nicht der Grund für den frühen Studienabbruch ist, eher finden sich Frühabbrecher unter den (noch unentschlossenen) Nicht-Berufstätigen oder den Vollzeit-Berufstätigen. 


\subsection{Analyse der Studienabbruchrate}

\section{Studienabbruchrate: Effekte in Abhängigkeit von Stamm- und Prüfungsdaten aus der Hörerevidenz}

Die Analyse des Studienabbruch-Risikos von Studierenden, die mindestens eine positive Leistung an der Wirtschaftsuniversität erbracht haben, erfolgte mit Hilfe des übergangsspezifischen semiparametrischen Modells von Cox. Als Studiendauer bis zum Studienabbruch wurde zur Analyse der in der Hörerevidenz verfügbaren Stamm- und Prüfungsdaten die Definition (b) - die Dauer bis zum Studienabbruch beträgt von Immatrikulierten, die an der Wirtschaftsuniversität keine einzige Leistung erbracht haben, ein Semester; bei anderen Studienabbrechern entspricht sie der Zeit (in Monaten) zwischen Studienbeginn und dem Datum der letzten Leistung plus ein Semester - herangezogen (vgl. Abschnitt 10.1). Studierende, die zum Stichtag das Studium bereits abgeschlossen haben bzw. noch studieren, gehen als rechts-zensierte Fälle in das Modell ein.

Tabelle 13.4 zeigt die Ergebnisse der Analyse der (inländischen) Immatrikulationsjahrgänge der Wintersemester 1989 bis 1993 unter Verwendung der Stammdaten aus der Hörerevidenz und Implementierung der Variablen analog zu den vorangegangenen Abschnitten. Die Überprüfung der funktionalen Form der kontinuierlichen Variablen ("matalt", "lag") zeigte, dass die gewählten Kategorien auch in dem übergangsspezifischen Modell für den Studienabbruch passend sind. Das Modell könnte wieder durch Zusammenfassung bzw. Reduzierung von Variablen kompakter angeschrieben werden. Bedeutende Interaktionseffekte konnten nicht beobachtet werden.

Die Ergebnisse liefern ein ähnliches Bild wie für den Studienabbruch ohne positive Leistung; siehe Tabelle 13.1. Beispielsweise weisen Immatrikulierte, die bei der Matura älter als 20 Jahre waren, (zu jedem Zeitpunkt $t \geq 6$ ) eine um $67 \%$ höhere Studienabbruchrate als die Referenzkategorie auf. Die größten Unterschiede ergeben sich bei den Schultypen; nur HTL-Maturanten zeigen in Tabelle 13.4 eine höhere Studienabbruchrate als AHS-Maturanten (Referenzkategorie; $p<0.0001$ ). Mit Hilfe von Interaktionsvariablen einzelner Variablen mit der Zeit wurde ergänzend untersucht, ob der Einfluss der einbezogenen Variablen mit der Zeit variiert. Es traten jedoch keine signifikanten Effekte auf.

Als Alternative wurde das Studienabbruch-Risiko von Immatrikulierten, die nach Definition (b) mindestens drei Semester an der Wirtschaftsuniversität studiert haben, analysiert. Dieses Modell liefert unter Einbeziehung der glei- 
Tabelle 13.4: Analyse der Studienabbruchrate auf Basis der Stammdaten aus der Hörerevidenz (Jahrgänge 1989 bis 1993)

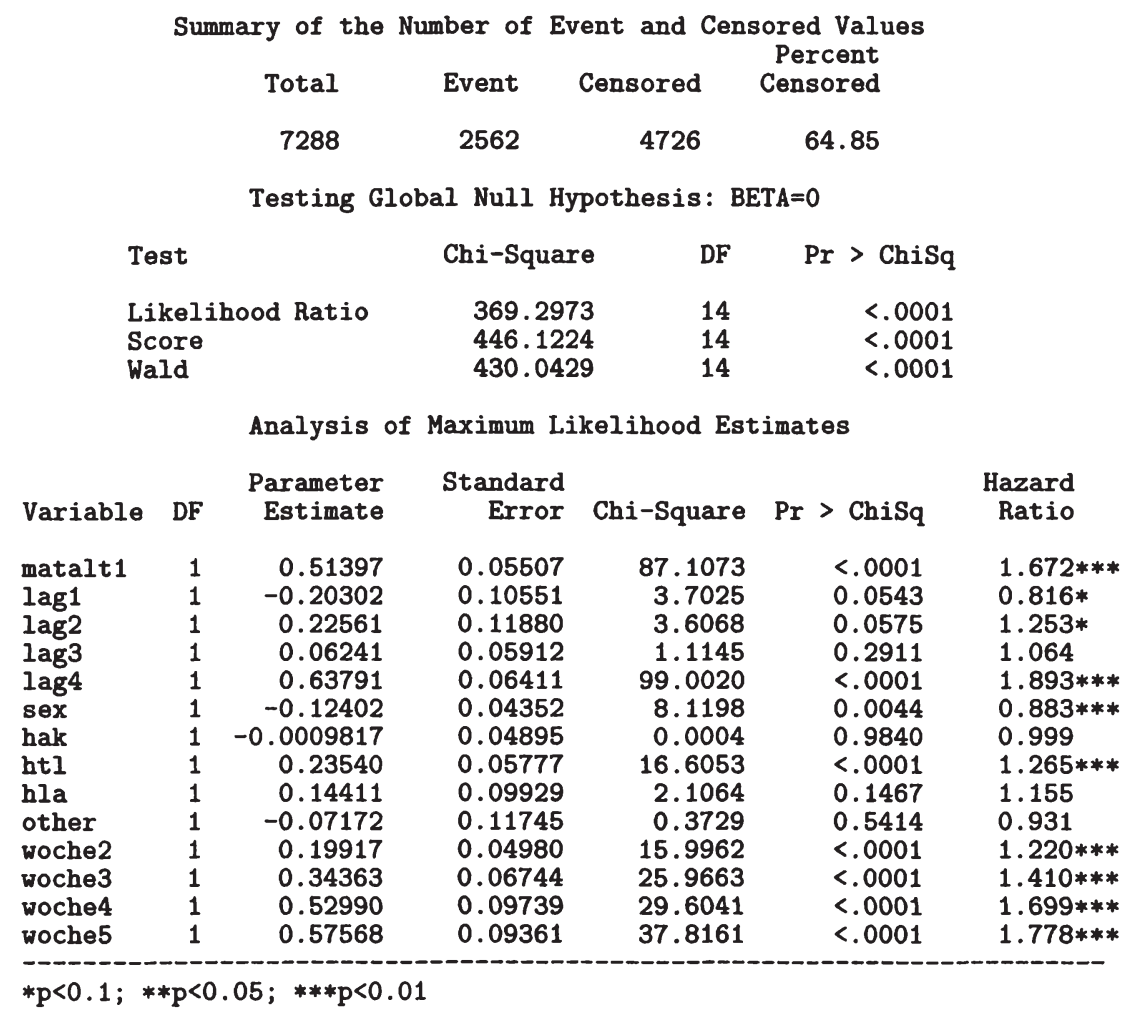

chen Variablen ähnliche Ergebnisse. Bezüglich der einbezogenen Variablen aus der Hörerevidenz unterscheiden sich somit sehr frühe Studienabbrecher nicht wesentlich von Studienabbrechern, die schon einige Leistungen an der Wirtschaftsuniversität erbracht haben.

In einem weiteren Schritt wurde untersucht, inwiefern sich die Leistungen des ersten Studienjahrs auf das Studienabbruch-Risiko auswirken. Analog zum vorangegangenen Kapitel wurden die Variablen "gesposj1" und "vpj1" in das semiparametrische Modell von Cox aufgenommen. Der Datensatz ist gegenüber dem vorangegangenen Modell um jene $(6 \%)$ Studierenden verringert worden, die zwar bis zum Stichtag mindestens eine positive Leistung abgelegt aber nach Definition (b) bis zum Studienabbruch weniger als zwei Semester an der Wirt- 
schaftsuniversität verbracht haben. Die Überprüfung der funktionalen Form von "gesposj1" auf Basis der Stammdaten und der Variablen "vpj1" zeigt, dass die lineare Funktion bis zur zehnten Prüfung passend ist. Ab der zehnten Prüfung ist der Abwärtstrend wie erwartet nicht mehr zu beobachten (siehe Abbildung 13.1).

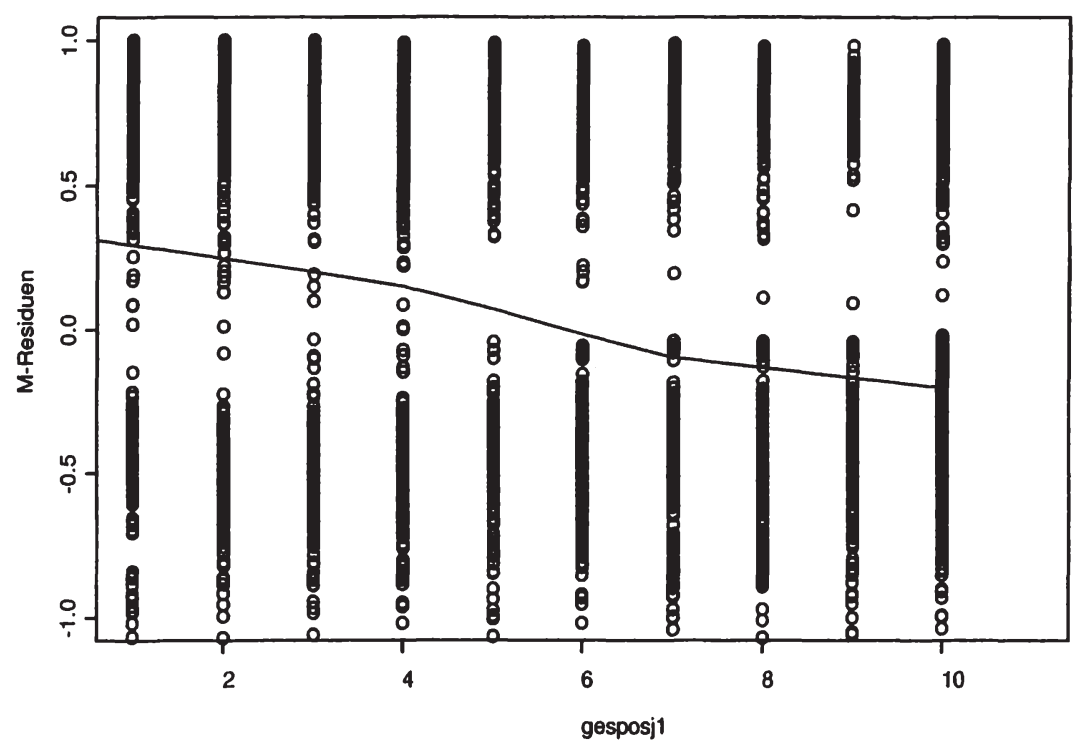

Abbildung 13.1: Plot der (geglätteten) Martingal-Residuen gegen die Variable "gesposj1" im übergangsspezifischen Cox-Modell für den Studienabbruch

Tabelle 13.5 zeigt das entsprechende Modell nach Einbeziehung der beiden Prüfungsvariablen und Reduzierung bzw. Zusammenfassung von Kategorien von Variablen.

Nach Einbeziehen der Prüfungsdaten verlieren wie erwartet die Immatrikulationswochen an Einfluss. Die Variable "spaet" fasst die Kategorien "woche4" und "woche5" zusammen. Die restlichen Variablen der Hörerevidenz wie das Alter bei der Matura, das Geschlecht und der HTL-Abschluss zeigen einen ähnlichen Einfluss wie vor der Einbeziehung der Prüfungsdaten. Ähnlich wie bei der Analyse der Studienabschlussrate verändert die Variable "hak" ihre Interpretation nach Einbeziehung der Prüfungsvariablen - ein HAK-Abschluss erhöht unter Berücksichtigung der Prüfungsvariablen die Studienabbruchrate. Die Erklärung hierfür ist wieder in der (für HAK-Absolventen) höheren Anzahl 
Tabelle 13.5: Analyse der Studienabbruchrate auf Basis der Stamm- und Prüfungsdaten aus der Hörerevidenz

Summary of the Number of Event and Censored Values

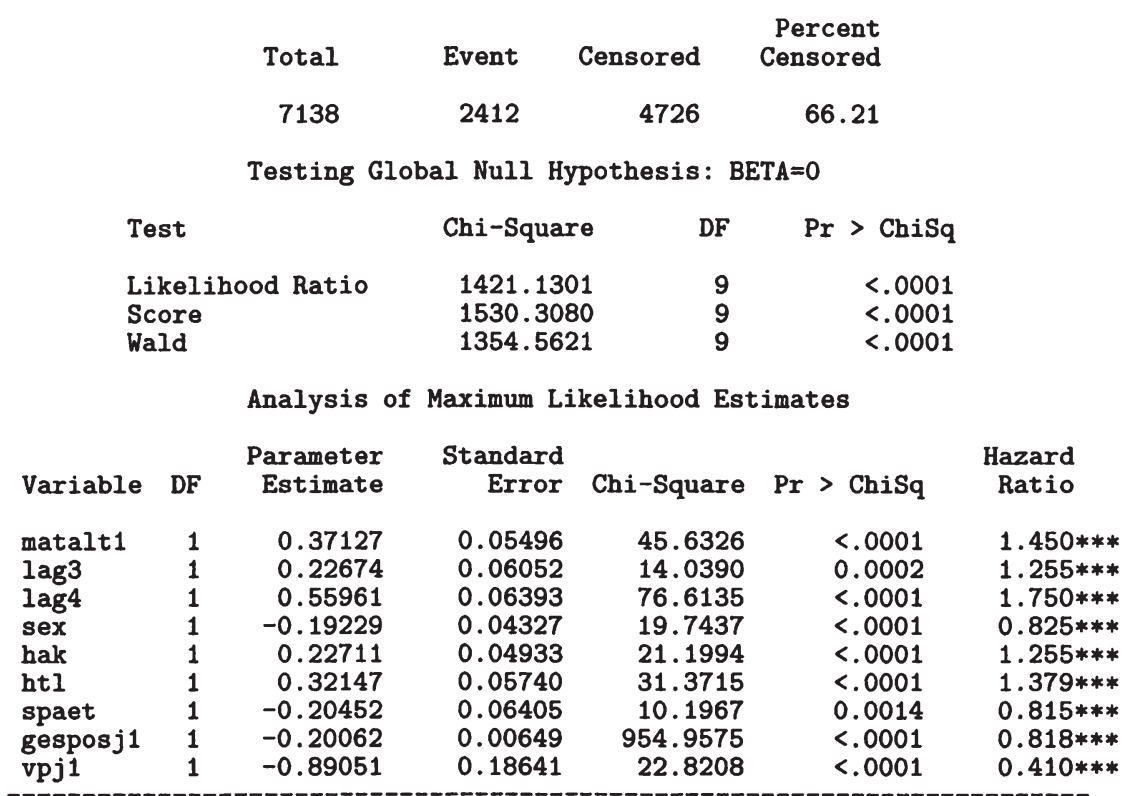

$* \mathrm{p}<0.1 ; * * \mathrm{p}<0.05 ; * * * \mathrm{p}<0.01$

der Prüfungserfolge (exklusive Ergänzungsprüfungen) im ersten Studienjahr zu suchen.

Die Leistungen des ersten Studienjahrs zeigen den erwarteten Effekt auf das Studienabbruch-Risiko:

- "gesposj1": Jede (bis zur zehnten) zusätzlich positiv abgelegte Prüfung im ersten Studienjahr verringert (zu jedem Zeitpunkt $t \geq 12$ ) das Studienabbruch-Risiko um 18\% (bei gleicher Ausprägung aller anderen Variablen).

- "vpj1": das Ablegen mindestens einer Vor- bzw. Teildiplomprüfung im ersten Studienjahr verringert das Studienabbruch-Risiko massiv (um etwa $59 \%)$.

Der Notendurchschnitt zeigt keinen zusätzlichen Effekt. 


\section{Studienabbruchrate: Effekte in Abhängigkeit von Daten der Stichproben-Population}

Ergänzend wurden wieder die aus der Befragung erhaltenen Daten der Jahrgänge 1990 und 1993 für die Analyse der Studienabbruchrate von Studierenden, die mindestens eine positive Leistung an der Wirtschaftsuniversität erbracht haben, herangezogen.

Zur Analyse der Befragungsdaten wurde die Definition (c) - die Studiendauer ist die Zeit zwischen Studienbeginn und dem auf Befragung angegebenen Datum des Studienabschlusses bzw. Studienabbruchs - verwendet. Es wurde auch überprüft, dass die Verwendung der Definition (b) für den Studienabbruch sehr ähnliche Ergebnisse liefert.

Nach Einbeziehung der Schul-Abschlussnoten und der Berufstätigkeit im ersten Semester (analog zu den vorangegangenen Abschnitten) sowie Reduzierung von Variablen aus der Hörerevidenz erhält man die Tabelle 13.6.

Bei der Interpretation der Tabelle 13.6 ist zu beachten, dass die Immatrikulationswochen bei der Reduzierung der Variablen aufgrund des geringen Erklärungsbeitrages nicht in das (finale) Modell aufgenommen worden sind. Die Variablen aus der Hörerevidenz zeigen nach Einbeziehung der (zeitunabhängigen) Variablen aus der Befragung die erwartete Richtung. Die Variablen aus der Befragung können wie folgt interpretiert werden:

- "mathe34", "englisch34": Schlechtere Abschlussnoten im Mathematik erhöhen das Studienabbruch-Risiko (bei mindestens einer erbrachten positiven Prüfungsleistung), während die Abschlussnoten aus Englisch keinen Effekt zeigen.

- "job12, job3“: Studierende, die im ersten Semester mindestens fünf und weniger als 30 Wochenstunden berufstätig waren ("job12" ist die Zusammenfassung der Kategorien "geringfügig" und "teilweise beschäftigt"), haben zu jedem Zeitpunkt $(t>6)$ eine um $50 \%$ höherer Studienabbruchrate als NichtBerufstätige. Vollzeit-Berufstätige haben noch ein höheres StudienabbruchRisiko. Diese Interpretation entspricht somit den deskriptiven Analyse aus Abschnitt 11.2.

Bezieht man in einem weiteren Schritt analog zu Abschnitt 12.3 die zeitabhängigen Variablen durchschnittliche Berufstätigkeit seit Studienbeginn ("empav") sowie die Anzahl der für eine zweite Ausbildung ("cumbildg") bzw. für Kinderbetreuung ("cumkind") investierten Semester (zu jedem Ereigniszeitpunkt) in das semiparametrische Modell ein, erhält man die Tabelle 13.7. 
Tabelle 13.6: Parameterschätzer des semiparametrischen Cox-Modells für den Studienabbruch unter Berücksichtigung von ergänzenden Variablen aus der Befragung

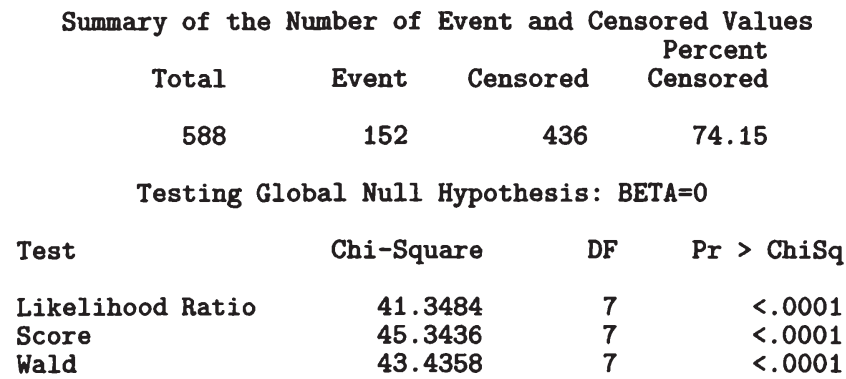

Analysis of Maximum Likelihood Estimates

$\begin{array}{lrrrrrr}\text { Variable } & \text { DF } & \begin{array}{r}\text { Parameter } \\ \text { Estimate }\end{array} & \begin{array}{r}\text { Standard } \\ \text { Error }\end{array} & \text { Chi-Square } & \text { Pr }>\text { ChiSq } & \begin{array}{c}\text { Hazard } \\ \text { Ratio }\end{array} \\ \text { matalt1 } & 1 & 0.28477 & 0.20565 & 1.9176 & 0.1661 & 1.329 \\ \text { sex } & 1 & -0.55842 & 0.18066 & 9.5537 & 0.0020 & 0.572 * * * \\ \text { ht1 } & 1 & 0.70494 & 0.20805 & 11.4810 & 0.0007 & 2.024 * * * \\ \text { mathe34 } & 1 & 0.33991 & 0.16800 & 4.0934 & 0.0431 & 1.405 * \\ \text { englisch34 } & 1 & -0.02090 & 0.17059 & 0.0150 & 0.9025 & 0.979 \\ \text { job12 } & 1 & 0.40359 & 0.18571 & 4.7230 & 0.0298 & 1.497 * * \\ \text { job3 } & 1 & 0.85096 & 0.22230 & 14.6531 & 0.0001 & 2.342 * * *\end{array}$

$* \mathrm{p}<0.1 ; * * \mathrm{p}<0.05 ; * * * \mathrm{p}<0.01$

Die Parameterschätzer der zeitkonstanten Variablen bleiben nach Einbeziehen der zeitabhängigen Variablen beinahe unverändert. Von den zeitveränderlichen Variablen ist es vor allem die durchschnittliche Berufsintensität, die einen massiven Einfluss auf die Studienabbruchrate zeigt. Jede Stunde, die durchschnittlich mehr seit Studienbeginn gearbeitet worden ist, bedeutet ein um etwa 3\% höheres Studienabbruch-Risiko. Mit der Anzahl der Semester, die in eine zweite Ausbildung investiert werden, nimmt die Studienabbruchrate ab. Die Variable "cumkind" zeigt keinen Einfluss auf die Hazardrate für den Studienabbruch. Allerdings zeigt Tabelle 11.21, dass nur sehr wenige Studienabbrecher in der Befragung angegeben haben, während ihrer WU-Studienzeit ein Kind betreut zu haben. 
Tabelle 13.7: Parameterschätzer des semiparametrischen Cox-Modells für den Studienabbruch unter Berücksichtigung von zeitabhängigen Variablen (Stichproben-Population)

Testing Global Null Hypothesis: BETA=0

$\begin{array}{lrrr}\text { Test } & \text { Chi-Square } & \text { DF } & \text { Pr }>\text { ChiSq } \\ \text { Likelihood Ratio } & 65.5162 & 9 & <.0001 \\ \text { Score } & 69.1857 & 9 & <.0001 \\ \text { Wald } & 65.1699 & 9 & <.0001\end{array}$

Analysis of Maximum Likelihood Estimates

\begin{tabular}{lrrrrrr} 
Variable & DF & $\begin{array}{r}\text { Parameter } \\
\text { Estimate }\end{array}$ & $\begin{array}{r}\text { Standard } \\
\text { Error }\end{array}$ & Chi-Square & Pr $>$ ChiSq & $\begin{array}{c}\text { Hazard } \\
\text { Ratio }\end{array}$ \\
matalt1 & 1 & 0.35981 & 0.20906 & 2.9622 & 0.0852 & $1.433 *$ \\
sex & 1 & -0.68803 & 0.19931 & 11.9173 & 0.0006 & $0.503 * * *$ \\
hak & 1 & -0.38979 & 0.23306 & 2.7972 & 0.0944 & $0.677 *$ \\
htl & 1 & 0.65263 & 0.23946 & 7.4277 & 0.0064 & $1.921 * * *$ \\
hlaother & 1 & 0.37468 & 0.31623 & 1.4038 & 0.2361 & 1.455 \\
mathe34 & 1 & 0.39870 & 0.17403 & 5.2485 & 0.0220 & $1.490 *$ \\
empav & 1 & 0.03147 & 0.00624 & 25.4656 & $<.0001$ & $1.032 * * *$ \\
cumbildg & 1 & -0.20767 & 0.12073 & 2.9585 & 0.0854 & $0.812 *$ \\
cumkind & 1 & 0.04160 & 0.10838 & 0.1473 & 0.7011 & 1.042 \\
\hline
\end{tabular}

$* \mathrm{p}<0.1 ; * * \mathrm{p}<0.05 ; * * * \mathrm{p}<0.01$ 


\section{Kapitel 14}

\section{Erweiterung der Analysen}

Die letzten beiden Kapitel zeigten ein breites Spektrum der Anwendungsmöglichkeiten der Ereignisanalyse für die Analyse der Studiendauer sowie des Studienabbruchs. In diesem Kapitel wird nach einer Sensitivitätsanalyse betreffend der Modellannahme eines bedingt unabhängigen Zensierungsmechanismus auf weitere Analysemöglichkeiten der vorliegenden Daten eingegangen. So können etwa je nach Fragestellung einzelne Studienphasen (Studieneingangsphase, erster und zweiter Studienabschnitt) getrennt voneinander (bzw. in Abhängigkeit zueinander) analysiert werden oder auch (insbesondere im Falle von Studienabbruchzeiten, die zumeist nicht exakt gemessen werden können) diskrete Modelle zur Anwendung kommen.

\subsection{Sensitivitätsanalyse}

Die Analysen der vorangegangenen Kapiteln sind unter der Annahme eines bedingt unabhängigen Zensierungsmechanismus erfolgt, das heißt, es wurde vorausgesetzt, dass die Dauer bis zum Studienabschluss (unter den einbezogenen Variablen) bedingt unabhängig von der Dauer bis zum Studienabbruch ist. Diese Annahme ist ausführlich in den Abschnitten 3.2 und 5.2 diskutiert worden.

Im Abschnitt 7.1 ist eine Möglichkeit vorgestellt worden, welche die Robustheit der Regressionskoeffizienten gegenüber Änderungen des Zensierungsmusters analysieren hilft. In Anlehnung an die dort beschriebene Methode wird zur Analyse der Robustheit der Parameterschätzer im parametrischen loglogistischen Modell für den Studienabschluss angenommen, dass alle Studienabbrecher zum Ende der Studie noch studieren; ihre Zensierungszeit wird 
somit gleich der Zeitdauer der Studie gesetzt. Tabelle 14.1 zeigt das Ergebnis basierend auf den Variablen aus Tabelle 12.7.

Tabelle 14.1: Sensitivitätsanalyse des log-logistischen Modells für die Reststudiendauer mit modifizierten Studienabbruch-Zeiten (Jahrgänge 1989 bis 1993)

Model Information

\author{
Number of Observations \\ Noncensored Values \\ Right Censored Values \\ Name of Distribution \\ Log Likelihood
}

7435

3034

4401

LLogistic

$-5671.55874$

Analysis of Parameter Estimates

\begin{tabular}{lrrrrrrr} 
Parameter & DF Estimate & $\begin{array}{c}\text { Standard } \\
\text { Error }\end{array}$ & \multicolumn{2}{c}{$\begin{array}{c}95 \% \text { Confidence } \\
\text { Limits }\end{array}$} & $\begin{array}{c}\text { Chi- } \\
\text { Square Pr }>\text { ChiSq }\end{array}$ \\
Intercept & 1 & 4.5935 & 0.1013 & 4.3949 & 4.7921 & 2054.31 & $<.0001$ \\
matalt1 & 1 & 0.2380 & 0.0443 & 0.1511 & 0.3248 & 28.84 & $<.0001 * * *$ \\
lag1 & 1 & 0.0270 & 0.0722 & -0.1144 & 0.1684 & 0.14 & 0.7083 \\
sex & 1 & 0.0820 & 0.0350 & 0.0134 & 0.1506 & 5.48 & $0.0192 * *$ \\
sex*ahs & 1 & 0.0598 & 0.0454 & -0.0292 & 0.1487 & 1.74 & 0.1877 \\
hak & 1 & 0.2270 & 0.0328 & 0.1626 & 0.2914 & 47.75 & $<.0001 * * *$ \\
ht1 & 1 & 0.2079 & 0.0466 & 0.1165 & 0.2993 & 19.87 & $<.0001 * * *$ \\
gesposj1 & 1 & -0.1518 & 0.0044 & -0.1604 & -0.1431 & 1182.01 & $<.0001 * * *$ \\
vpj1 & 1 & -0.4038 & 0.0423 & -0.4866 & -0.3210 & 91.30 & $<.0001 * * *$ \\
average & 1 & 0.1423 & 0.0219 & 0.0994 & 0.1851 & 42.33 & $<.0001 * * *$ \\
Scale & 1 & 0.4658 & 0.0072 & 0.4520 & 0.4801 & & \\
- & 1 & 0.460 &
\end{tabular}

$* \mathrm{p}<0.1 ; * * \mathrm{p}<0.05 ; * * * \mathrm{p}<0.01$

Mit dieser Modellierung werden nun alle Studierenden, die in den ersten acht Semestern des Studiums abgebrochen und daher zuvor nicht in das Modell einbezogen worden sind, berücksichtigt. Daher erhöht sich auch die Zahl der rechts-zensierten Fälle von 2432 auf 4401.

Die Konfidenzintervalle der Regressionskoeffizienten der beiden Modelle überlappen für alle einbezogenen Kovariablen. Zumeist wurden die Effekte der Variablen etwas stärker, wie etwa der Einfluss des Alters bei der Matura ("matalt") und der Anzahl der positiven Leistungen im ersten Studienjahr ("gesposj1"). Beide Variablen sind auch Einflussgrößen, die das StudienabbruchRisiko erhöhen, und daher konnte erwartet werden, dass in dem in Tabelle 14.1 angeführten Extremfall der Einfluss etwas verstärkt wird. Im Allgemeinen kann jedoch geschlossen werden, dass die Regressionskoeffizienten der einbezogenen Variablen ziemlich robust gegenüber Änderungen des Zensierungsmechanismus sind. Semiparametrische Modellierungen liefern vergleichbare Aussagen. 
Auffallend ist jeweils der bedeutende Unterschied der Parameter "Intercept" $\left(\beta_{0}\right)$ und "Scale" $(\sigma)$ zwischen den beiden Modellen. Diese beiden Parameterschätzer, die die Gestalt der Verteilung bestimmen, hängen weitaus mehr von den Zensierungszeiten ab. Obwohl sich die Regressionskoeffizienten der einzelnen Kovariablen nur leicht verändert zeigen, hat sich der geschätzte Median der (übergangsspezifischen) Reststudiendauer der einzelnen Studentengruppen durch die veränderte Modellierung der Abbruchzeiten wie erwartet stark erhöht.

\subsection{Mehr-Episoden-Modelle}

In Erweiterung der Analysen des Kapitels 12 kann das Studium in mehrere Studienphasen geteilt werden. Dabei kann beispielsweise

- die Gesamtstudiendauer in Abhängigkeit der Dauer bis zur ersten Vor- bzw. Teildiplomprüfung (und anderer Kovariablen),

- die Gesamtstudiendauer in Abhängigkeit der Dauer des ersten Studienabschnitts,

- die Dauer des zweiten Studienabschnitts in Abhängigkeit der Dauer des ersten Studienabschnitts, oder auch

- die Dauer des ersten Studienabschnitts in Abhängigkeit der vorhandenen Daten aus der Hörerevidenz

modelliert werden. In den ersten beiden Fällen wird die Gesamtstudiendauer in Abhängigkeit des Eintreffens eines davor liegenden Ereignisses (der ersten Vor- bzw. Teildiplomprüfung, bzw. des ersten Studienabschnitts) analysiert. Es werden daher nur jene Studierenden in das Modell einbezogen, die dieses Ereignis bereits gehabt haben, das heißt, es handelt es um ein Modell mit links abgeschnittenen Daten (siehe Abschnitt 3.2). Von den einbezogenen Studierenden ist bekannt, dass sie bis zum Eintreffen dieses Zwischenereignisses nicht dem "Risiko" des Studienabschlusses ausgesetzt waren. Tabelle 14.2 zeigt als Beispiel die Analyse der Gesamtstudiendauer in Abhängigkeit der Dauer des ersten Studienabschnitts und der zeitabhängigen Variablen "Berufstätigkeit", "zweite Ausbildung" und "Kinderbetreuung" im zweiten Studienabschnitt (analog $\mathrm{zu}$ den beiden vorangegangenen Kapiteln). Mit diesem Modell wird einerseits eine Bestätigung gesucht, dass die Dauer des ersten Studienabschnitts einen starken Einfluss auf die Studienabschlussrate hat, da 
zu erwarten ist, dass die beiden Zeitdauern stark positiv korrelieren. Andererseits wird der Frage nachgegangen, inwiefern die einbezogenen zeitabhängigen Variablen die Dauer des zweiten Studienabschnitts (und damit die Gesamtstudiendauer) beeinflussen.

Tabelle 14.2: Analyse des zweiten Studienabschnitts anhand von links abgeschnittenen Daten (Stichproben-Population)

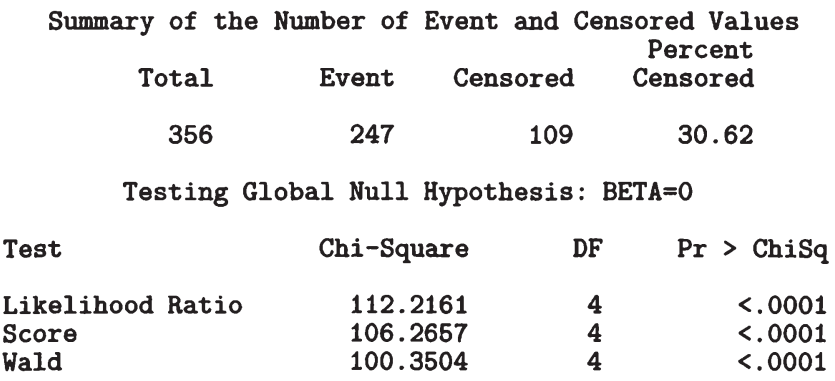

\begin{tabular}{|c|c|c|c|c|c|c|}
\hline \multirow[b]{2}{*}{ Variable } & \multicolumn{5}{|c|}{ Analysis of Maximum Likelihood Estimates } & \multirow[b]{2}{*}{$\begin{array}{r}\text { Hazard } \\
\text { Ratio }\end{array}$} \\
\hline & DF & $\begin{array}{r}\text { Parameter } \\
\text { Estimate }\end{array}$ & $\begin{array}{r}\text { Standard } \\
\text { Error }\end{array}$ & Chi-Square & $\operatorname{Pr}>\mathrm{ChiSq}$ & \\
\hline $\begin{array}{l}\text { dur1b } \\
\text { empav } \\
\text { cumbildg } \\
\text { cumkind }\end{array}$ & $\begin{array}{l}1 \\
1 \\
1 \\
1\end{array}$ & $\begin{array}{l}-0.05222 \\
-0.01877 \\
-0.03271 \\
-0.15236\end{array}$ & $\begin{array}{l}0.00584 \\
0.00644 \\
0.06320 \\
0.06201\end{array}$ & $\begin{array}{r}79.8697 \\
8.5005 \\
0.2679 \\
6.0367\end{array}$ & $\begin{array}{l}<.0001 \\
0.0036 \\
0.6047 \\
0.0140\end{array}$ & $\begin{array}{l}0.949 * * * * \\
0.981 * * * \\
0.968 \\
0.859 * *\end{array}$ \\
\hline
\end{tabular}

Die Ergebnisse zeigen, dass die Dauer des ersten Studienabschnitts einen massiven Einfluss (in erwarteter Richtung) auf die Gesamtstudiendauer hat. Unter Konstanthaltung der Studiendauer des ersten Studienabschnitts zeigen die durchschnittliche Berufstätigkeit und die Anzahl der Semester, in denen auch Kinder betreut wurden, einen deutlichen Einfluss (in erwarteter Richtung) auf die Studienabschlussrate.

Auch für die Analyse des Studienabbruchs sind ähnliche Mehr-Episoden-Modelle denkbar - etwa das Risiko des Studienabbruchs im zweiten Studienabschnitt in Abhängigkeit von erklärenden Variablen.

\subsection{Diskrete Modelle}

Die Dauer bis zum Studienabbruch kann über Definition (b) - die Dauer bis zum Studienabbruch beträgt von Immatrikulierten, die an der Wirtschaftsuni- 
versität keine einzige Leistung erbracht haben, ein Semester; bei anderen Studienabbrechern entspricht sie der Zeit (in Monaten) zwischen Studienbeginn und dem Datum der letzten Leistung plus ein Semester - nur approximiert werden. Als Alternative zu den kontinuierlichen Modellen aus Kapitel 13 können diskrete Modelle verwendet werden, in den die Zeitdauer bis zum Studienabbruch (bzw. Studienabschluss) in Semestern angegeben wird. Tabelle 14.3 zeigt die Analyse des Studienabbruchs mit Hilfe eines gruppierten Cox-Modells (siehe Abschnitt 8) auf Basis der Stamm- und Prüfungsdaten der Hörerevidenz.

Tabelle 14.3: Analyse der Studienabbruch-Wahrscheinlichkeit auf Basis des gruppierten Cox-Modells

Analysis of Parameter Estimates

\begin{tabular}{lrrrrrrr} 
Parameter & DF Estimate & $\begin{array}{c}\text { Standard } \\
\text { Error }\end{array}$ & $\begin{array}{r}95 \% \text { Confidence } \\
\text { Limits }\end{array}$ & $\begin{array}{c}\text { Chi- } \\
\text { Square Pr > ChiSq }\end{array}$ \\
Intercept & 1 & -2.7137 & 0.0459 & -2.8037 & -2.6238 & 3498.00 & $<.0001$ \\
matalt1 & 1 & 0.3703 & 0.0550 & 0.2626 & 0.4780 & 45.38 & $<.0001 * * *$ \\
lag3 & 1 & 0.2091 & 0.0605 & 0.0906 & 0.3276 & 11.96 & $0.0005 * * *$ \\
lag4 & 1 & 0.5329 & 0.0639 & 0.4077 & 0.6581 & 69.61 & $<.0001 * * *$ \\
gesch & 1 & -0.1758 & 0.0432 & -0.2605 & -0.0910 & 16.52 & $<.0001 * * *$ \\
hak & 1 & 0.2249 & 0.0493 & 0.1282 & 0.3215 & 20.80 & $<.0001 * * *$ \\
ht1 & 1 & 0.3134 & 0.0574 & 0.2008 & 0.4259 & 29.77 & $<.0001 * * *$ \\
spaet & 1 & -0.1926 & 0.0640 & -0.3182 & -0.0671 & 9.05 & $0.0026 * * *$ \\
gesposj1 & 1 & -0.1968 & 0.0065 & -0.2095 & -0.1842 & 925.88 & $<.0001 * * *$ \\
vpj1 & 1 & -0.9039 & 0.1864 & -1.2691 & -0.5386 & 23.52 & $<.0001 * * *$ \\
\hline
\end{tabular}

$* \mathrm{p}<0.1 ; * * \mathrm{p}<0.05 ; * * * \mathrm{p}<0.01$

Die Ergebnisse sind praktisch ident zu den Ergebnissen aus Tabelle 13.5. Die Ergebnisse erweisen sich auch als robust gegenüber weiteren Alternativen für die Dauer bis zum Studienabbruch (bzw. Studienabschluss). Nimmt man etwa die Anzahl der insgesamt an der Wirtschaftsuniversität inskribierten Semester als Studiendauer und definiert man den Studienabbruch entsprechend der Eintragung in der Hörerevidenz (siehe Abschnitt 10.1, Definition (A) für den Studienabbruch), so verändern sich die Parameterschätzer kaum.

Werden nur zeitunabhängige Kovariablen in das Modell einbezogen, so sind die unterschiedlichen Rechenzeiten zwischen dem kontinuierlichen und dem diskreten Modell (auch bei größeren Datenmengen wie in Tabelle 14.3) kaum von Bedeutung. Zieht man allerdings auch zeitabhängige Kovariablen in das Modell ein, so ist die Rechenzeit für diskrete Modelle (vor allem bei größeren Datenmengen und/oder mehreren zeitabhängigen Variablen) geringer. In diesem Fall muss der Nachteil des Informationsverlustes durch Diskretisierung von kontinuierlichen Daten und der Vorteil einer geringeren Rechenzeit gegeneinander abgewogen werden. 


\section{Kapitel 15}

\section{Zusammenfassung und Diskussion der Ergebnisse}

Die empirischen Analysen der vorliegenden Arbeit setzen sich insbesondere mit der Bestimmung von Merkmalen, die einen Effekt auf die Studiendauer und das Risiko eines Studienabbruchs ausüben, und weniger mit Kennzahlen des Studiums zusammen. Dieses Kapitel fasst die wichtigsten Ergebnisse dieser empirischen Analysen zusammen und gibt im letzten Abschnitt Ideen, wie die erzielten Ergebnisse bzw. die in der Hörerevidenz vorhandene Daten zu Studierenden zukünftig in Entscheidungsprozessen eine Hilfestellung bieten können.

\subsection{Analyse der Studiendauer und der Ha- zardrate für den Studienabschluss}

- Eingangsvoraussetzungen in Bezug auf den schulischen Werdegang:

- HAK-Absolventen haben eine kürzere Studiendauer als die Absolventen von anderen Schultypen (insbesondere als HTL- und männliche AHS-Absolventen) zu erwarten. Die Variable Schultyp korreliert mit den Leistungen des ersten Studienjahres. HAK-Absolventen absolvieren im ersten Studienjahr durchschnittlich zwei bis drei positive Lehrveranstaltungsprüfungen mehr als die Absolventen anderer Schultypen. Dies lässt sich auch auf das Wegfallen der Ergänzungsprüfungen zurückführen.

- Studierende, die bei Schulabschluss älter als 20 Jahre waren, haben 
eine längere Studiendauer als andere. Dies lässt den Schluss zu, dass eine Verzögerung beim Schulabschluss auch auf eine Verzögerung im Studium hinweist. Angesichts der geringen Anzahl von Studierenden aus dieser Gruppe kommt dieser Variablen nicht so große Bedeutung $\mathrm{zu}$.

- Studierende, die die Matura zu einem Nachtermin absolviert haben, weisen eine längere Studiendauer auf als andere. Auch dies deutet daraufhin, dass sich schwächere Leistungen in der Schule (bzw. im Jahr der Matura) negativ auf die Studienleistungen auswirken. Weiters ist zu erwähnen, dass Studierende, die die Matura zum Nachtermin Ende September bzw. Anfang Oktober absolvieren, erst Ende der Immatrikulationsfrist immatrikulieren können. Die späte Immatrikulation wiederum kann sich negativ auf die Studieneingangsphase auswirken.

- Studierende, die die Schule in Mathematik und Englisch mit Eins oder Zwei abgeschlossen haben, zeigen eine geringere Studiendauer als andere.

Insgesamt zeigen die einbezogenen Variablen einen erkennbaren $\mathrm{Zu}$ sammenhang zwischen Schul- und Studienleistungen.

- Zum Engagement für das Studium:

- Je später innerhalb der Immatrikulationsfrist immatrikuliert wird, desto länger ist die zu erwartende Studiendauer. Der massive Einfluss dieser Variablen lässt sich dadurch erklären, dass

- es meistens die Unentschlossenen (bzw. auf eine andere Chance Wartenden) sind, die am Ende der Immatrikulationszeit immatrikulieren, und dass

- eine späte Immatrikulation (schon während der Studienzeit) einen Startnachteil mit sich bringt. Studierende, die spät immatrikulieren, erhalten manchmal keinen Lehrveranstaltungsplatz mehr, haben die ersten Lehrveranstaltungseinheiten schon versäumt und finden sich dann langsamer auf der Universität zurecht.

Diesen Erklärungen entsprechend korreliert diese Variable stark mit den erbrachten Leistungen im ersten Studienjahr.

- Je größer die Anzahl der Prüfungserfolge und je besser der Notendurchschnitt im ersten Studienjahr umso geringer ist die Studiendauer. Dies zeigt, wie wichtig das erste Studienjahr (bzw. eine Studieneingangsphase) für den weiteren Studienerfolg ist. Die einbezogenen Prüfungsvariablen zeigen den erwartet hohen prädiktiven Charakter für die Studiendauer, daher eignen sie sich auch gut für frühe Prognosemodelle für die Studiendauer. 
- Zum sozialen Umfeld:

- Die Berufstätigkeit im ersten Semester zeigt, dass Studierende, die schon im ersten Semester mindestens 30 Stunden berufstätig sind, eine weitaus geringere Studienabschlussrate aufweisen. Weit aussagekräftiger ist die zeitabhängige Variable Berufstätigkeit. Je höher die durchschnittliche (und auch die laufende) Berufsintensität seit Studienbeginn umso länger ist die Studiendauer. Da das Berufsausmaß an der Wirtschaftsuniversität (wie in Abschnitt 11.2 gezeigt) relativ hoch ist, kommt dieser Variablen eine große Bedeutung zu.

Berufstätige Studierende brauchen zwar je nach Berufsintensität länger für das Studium. In den meisten Fällen macht sich jedoch diese Studienverzögerung bezahlt, weil nach Information des Zentrums für Berufsplanung (an der Wirtschaftsuniversität Wien) Absolventen mit Berufserfahrung größere Chancen auf einen Job haben als Studierende, die in Mindestzeit studiert und keine (oder kaum) Berufserfahrung haben.

- Je mehr Semester für alternative Ausbildungen (wie Doppelstudium, Lehrgang, etc.) und für Kinderbetreuung investiert wurden, desto höher ist die zu erwartende Studiendauer.

Insgesamt kann zusammengefasst werden, dass die demographischen Variablen aus der Hörerevidenz allein keinen großen Einfluss auf die Studiendauer zeigen. Prüfungsdaten des ersten Studienjahrs und die zusätzlich einbezogenen Variablen hingegen zeigen einen massiven Effekt auf die Studiendauer.

\subsection{Analyse der Studienabbruchrate}

Die deskriptiven Analysen aus Abschnitt 11.1 zeigen, dass ein Großteil der Studienabbrecher das Studium an der Wirtschaftsuniversität in einem sehr frühen Stadium abbrechen, viele davon überhaupt, ohne eine einzige (positive) Leistung erbracht zu haben; daher ist die Frage zu stellen, ob solche Studienanfänger nicht eher als "Schnupperstudierende" denn als Studierende der Wirtschaftsuniversität zu klassifizieren sind. Im Lichte dieser Fakten sind auch Kennzahlen über Abbruchquoten an der Wirtschaftsuniversität zu beurteilen.

In der vorliegenden Arbeit war es auch ein Ziel, zwischen frühen und späten Studienabbrüchen zu unterscheiden und unterschiedliche Einflussgrößen zu bestimmen. Es hat sich gezeigt, dass diese Fragestellung mit dem vorhandenen Datenmaterial nur schwer zu beantworten ist. Zumeist zeigen die einbezogenen Variablen keinen großen Unterschied zwischen Studienabbrüchen ohne positive 
Leistung (bzw. in den ersten Semestern) und späteren Studienabbrüchen. Am ehesten könnte noch die Berufstätigkeit erwähnt werden, die mit zunehmender Studiendauer eine größere Rolle spielt, wobei eine Vollzeit-Beschäftigung zu Studienbeginn auch einen massiven Einfluss auf einen frühen Studienabbruch zeigt. Auch die Schulnote aus Mathematik zeigt zu Beginn keinen Einfluss, bei späteren Studienabschlüssen hingegen wirken sich schlechtere Noten erhöhend auf das Studienabbruch-Risiko aus. Weiters zeigt Tabelle 11.8, dass die vorwiegende Beschäftigung nach dem Studienabbruch zwischen frühen und späteren Studienabbrechern variiert.

Ganz allgemein konnten die folgenden Einflussgrößen auf das StudienabbruchRisiko identifiziert werden:

- HTL-Absolventen weisen ein höheres Studienabbruch-Risiko als die Absolventen der anderen Schultypen auf, HAK-Absolventen ein etwas geringeres.

- Studierende, die bei Schulabschluss älter als 20 Jahre waren, haben eine höhere Abbruchwahrscheinlichkeit als andere.

- Je später innerhalb der Immatrikulationsfrist immatrikuliert wird, desto höher wird das Studienabbruch-Risiko geschätzt. Diese Variable korreliert stark mit den erbrachten Leistungen im ersten Studienjahr.

- Je größer die Anzahl der Prüfungserfolge im ersten Studienjahr, umso geringer ist in den darauffolgenden Studienjahren das Studienabbruch-Risiko. Die beiden einbezogenen Variablen zeigen den erwartet starken Effekt auf die Wahrscheinlichkeit eines Studienabbruchs.

- Je höher die durchschnittliche Berufsintensität umso größer ist das Studienabbruch-Risiko. Auch die als zeitabhängige Variable modellierte Berufstätigkeit zeigt den erwartet hohen Effekt.

- Ebenfalls erhöhend auf das Studienabbruch-Risiko wirkt sich die Betreuung von Kindern neben dem Studium aus.

\subsection{Ausblick}

In der vorliegenden Arbeit bildet die Anwendung der Ereignisanalyse auf Studienverlaufsdaten von Immatrikulationjahrgängen den Schwerpunkt. Die Verfahren der Ereignisanalysen eignen sich, die Verteilung der Studiendauer und ihre Charakteristika wie die mediane Studiendauer zu schätzen. Durch das Einbeziehen von erklärenden Variablen kann darüber hinaus der Effekt von 
bestimmten Merkmalen auf die Studiendauer und das Studienabbruch-Risiko quantifiziert werden.

Die Modellierung von Studiendauer und Studienabbruch-Risiko unter Verwendung von Erklärungsfaktoren erlaubt es, schon in den ersten Semestern fundierte Aussagen über die Entwicklung eines Jahrgangs zu machen; die Modelle liefern Prognosen über die Studiendauer und Abbruchwahrscheinlichkeit von Teilpopulationen der Studierenden bis zu Individualprognosen. Solche Prognosen sind unter anderem auch ein wichtiges Instrument zur frühen Beurteilung von Maßnahmen, die helfen sollen, die Studiendauer bzw. die Abbruchquote zu reduzieren. Manche Erklärungsfaktoren sind in den universitären Datenbeständen verfügbar und können daher für routinemäßige Analysen genützt werden, andere müssen aus anderen Quellen erhoben werden.

Die in der vorliegenden Arbeit präsentierten Erklärungsmodelle können Teil eines umfassenden Systems zur Beschreibung des Studierverhaltens an der Wirtschaftsuniversität sein. Hackl und Sedlacek (2002) schlagen in ihrem Forschungsbericht vor, "einerseits ein Kennzahlensystem "Studierende und Studienfortschritte" zu führen, andererseits von Zeit zu Zeit die Erklärungsmodelle an neuere Daten anzupassen".

Das vorgeschlagene Kennzahlensystem umfasst Kennzahlen zu den Erstinskribenten, den Studierenden, den Studienabbrechern und den Absolventen eines Berichtsjahres. Alle Statistiken können auf Basis der universitären Datenbeständen routinemäßig ermittelt werden. Derartige Kennzahlen sind ein Instrument für die Bewertung der bestehenden Situation und die Wahl und Einschätzung von zu treffenden Maßnahmen. Sie sollen helfen, den Lehr- und Lernbetrieb an der Wirtschaftsuniversität zu verbessern. Sie sind für unterschiedliche Personengruppen von Interesse: für die Studierenden, für die Lehrenden, für die für den Lehrbetrieb Verantwortlichen, für die Geldgeber. 


\section{Anhang A}

\section{Schulformen}

01 Gymnasium

02 Humanistisches Gymnasium

03 Neusprachliches Gymnasium

04 Realistisches Gymnasium

05 Realgymnasium

06 Naturwissenschaftliches Realgymnasium

07 Mathematisches Realgymnasium

08 Oberstufenrealgymnasium

09 Wirtschaftskundliches Realgymnasium

10 Realschule

11 Frauenoberschule

12 Aufbaugymnasium

13 Aufbaurealgymnasium

14 Aufbaumittelschule

15 Gymnasium für Berufstätige

16 Realgymnasium für Berufstätige

17 Arbeitermittelschule

18 Berufsreifeprüfung

19 Höhere technische und gewerbliche Lehranstalt

20 Handelsakademie

21 Höhere Lehranstalt für wirtschaftliche Berufe

22 Lehrerbildungsanstalt

23 Höhere land- und forstwirtschaftliche Lehranstalt

24 Studienberechtigungsprüfung

25 Ausländische Reifeprüfung

26 BA für Sozialpädagogik

27 Externistenreifeprüfung 
28 Bundesanstalt für Kindergartenpädagogik

29 Akademie

30 Inländische postsekundäre Bildungseinrichtung

31 Ausländische postsekundäre Bildungseinrichtung

98 Reifeprüfung nicht relevant

99 Keine Reifeprüfung 


\section{Anhang B}

\section{Fragebogen}

Liebe Kolleginnen und Kollegen!

Im Rahmen der Diskussion um die Ursachen von langen Studienzeiten unserer Absolventen und hohen Studienabbruchraten hat die Universitätsleitung der Wirtschaftsuniversität Wien ein Projekt in Auftrag gegeben, in dessen Rahmen Studienverläufe an der WU analysiert werden. Die empirische Analyse wird im Rahmen einer Dissertation durchgeführt, die vom Institut für Statistik betreut wird.

Der Studienverlauf jedes und jeder einzelnen Studierenden wird sehr von auBeruniversitären Faktoren - wie etwa Erwerbstätigkeit neben dem Studium beeinflusst. Daher sind entsprechende (Längsschnitt-)Daten für die Analyse von Studienverläufen immens wichtig. Wir treten an Sie als Angehörige(r) der Immatrikulationsjahrgänge 1990 und 1993 heran und ersuchen Sie um Ihre Mitarbeit, indem Sie den beiliegenden Fragebogen ausfüllen und zurückschicken.

Wir benötigen für unsere Analyse Ihre persönlichen Daten und wollen diese auch mit Ihren Studiendaten zusammenführen, die uns die Universitätsverwaltung zur Verfügung gestellt hat. Uns ist klar, dass Ihre Mitarbeit ein großes Vertrauen in unser Verantwortungsbewusstsein beim Behandeln Ihrer persönlichen Daten erfordert. Wir ersuchen Sie, uns dieses Vertrauen entgegenzubringen und damit eine Arbeit zu ermöglichen, von der wir hoffen, dass sie zum besseren Funktionieren der WU beitragen wird.

Die Ergebnisse der Analysen beziehen sich nicht auf Einzelpersonen, sondern stets auf aggregierte Datensätze. Daher ist aus den veröffentlichten Ergebnissen die Identifikation von Personen unmöglich.

Gerne schicken wir Ihnen nach Abschluss der Untersuchung eine Zusammen- 
fassung der Ergebnisse. Bitte, nehmen Sie sich 10 Minuten Zeit und senden Sie den ausgefüllten Fragebogen möglichst bis 17.November an uns zurück. Für Ihre Mitarbeit danken wir Ihnen und grüßen sehr freundlich

\author{
Univ. Prof. Dr. Peter Hackl Dipl.-Ing. Günther Sedlacek
}




\section{Fragebogen}

Wir versichern Ihnen, dass sensible Daten, die hier abgefragt werden, anonym behandelt und entsprechend dem Datenschutzgesetz nicht weitergegeben werden.

1. Matrikelnummer:

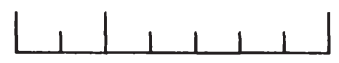

2. Geschlecht:

Weiblich

Männlich

3. Noten im Maturazeugnis:

Mathematik:

Englisch:

4. Welche höchste abgeschlossene Schulbildung hat/hatte Ihr Vater/Ihre Mutter?

Pflichtschulabschluss

Vater

Mutter

Lehre

Matura

Hochschulabschluss

Trifft nicht zu

5. a) Haben Sie Kinder?

$\square \mathrm{Ja} \quad \square$ Nein

b) (Wenn ja:) In welchem Zeitraum lebten Sie mit Ihren Kindern in Ihrem eignen Haushalt?

gar nicht
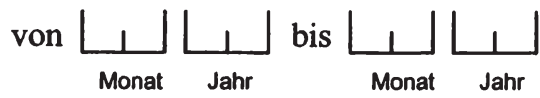

6. Hatten Sie zu Beginn des WU-Studiums die Absicht, es auch abzuschließen?

$\square$ Ja $\quad \square$ Nein $\square$ War nicht sicher

7. a) Ist Ihr WU-Studium
$\square$ abgeschlossen
abgebrochen
$\square$ im Gange ? 
Wenn abgebrochen, beantworten Sie bitte b) und c)

b) Zeitpunkt des Studienabbruchs

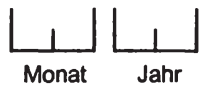

c) Welcher Hauptaktivität sind Sie im Jahr nach Ihrem WU-Studienabbruch nachgegangen? Geben Sie auch bitte an, ob diese Tätigkeit mit Bezug zu ihrem vorangegangenen $W U$-Studium war oder nicht.

mit Bezug ohne Bezug

$\square \quad$ Universitätsstudium außerhalb der WU:

alternative Ausbildung (FH, College, etc.):

Erwerbstätigkeit

Wenn noch im Gange:

d) Haben Sie noch vor, Ihr WU-Studium abzuschließen?

$\square$ Ja $\square$ Nein $\square$ Weiß nicht

8. Besuchen oder besuchten Sie, abgesehen von der WU, während Ihrer WUStudienzeit (mindestens zwei Semester lang) weitere Bildungsangebote?

Ja $\square$ Nein

Wenn ja, füllen Sie bitte zum zeitaufwendigsten Bildungsangebot, die folgenden Felder aus:

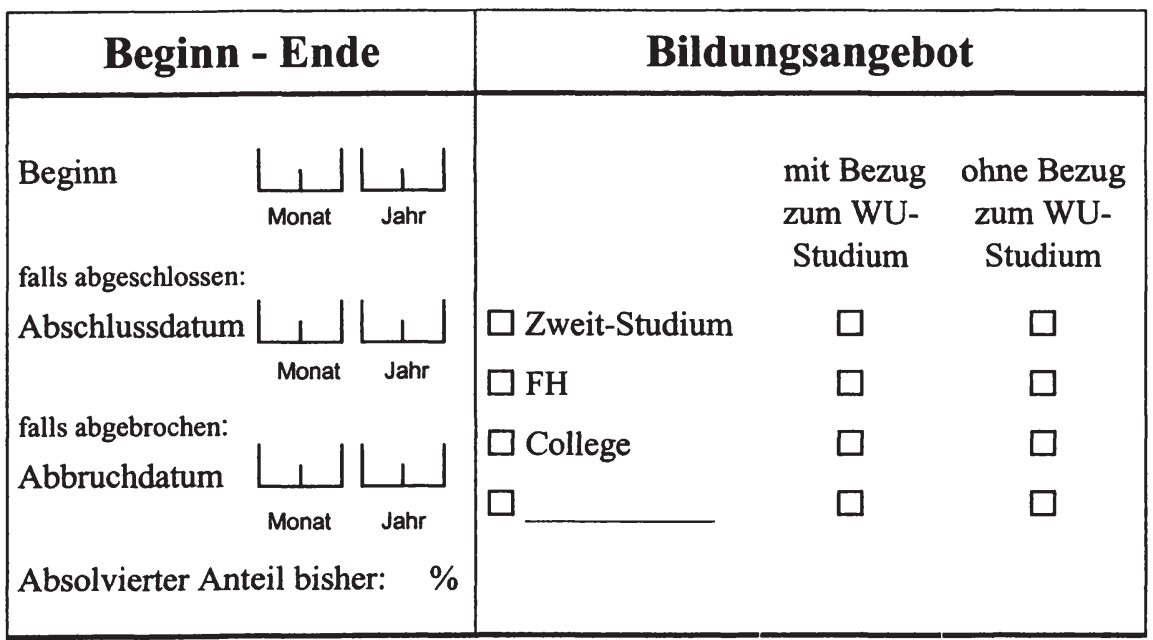


9. a) Haben Sie - als Bestandteil Ihres WU-Studiums - Zeit im Ausland verbracht, um zu arbeiten oder zu studieren?

\section{$\mathrm{Ja} \square$ Nein}

b) Wenn ja, geben Sie bitte

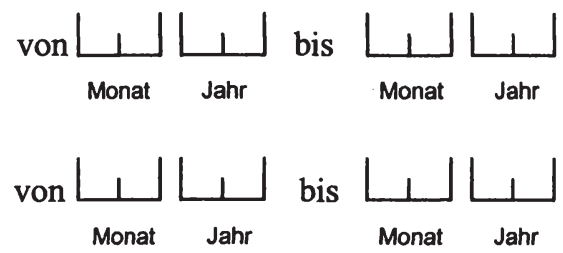

10. In welchem durchschnittlichen Ausmaß waren Sie im Jahr vor Beginn Ihres WU-Studiums erwerbstätig?

gar nicht

$\square$ weniger als 20 Wochenstunden

$\square$ weniger als 30 Wochenstunden

$\square$ mehr als 30 Wochenstunden

11. a) In welchen Jahren Ihres WU-Studiums sind Sie während des Studienjahres einer Erwerbstätigkeit nachgegangen (exklusie Erwerbstätigkeiten, die ausschließlich in der lehrveranstaltungsfreien Zeit durchgeführt worden sind)? (Durchschnittliches Wochenstundenausma $B$ bitte in Zahlen angeben)

Beispiel:

\begin{tabular}{|l|c|c|c|c|c|c|c|c|c|}
\hline Studienjahr & 1 & 2 & 3 & 4 & 5 & 6 & 7 & 8 &. \\
\hline Wochenstunden & 1 & 1 & 10 & 10 & 20 & 20 & 15 & 1 & $\ldots$ \\
\hline
\end{tabular}

\begin{tabular}{|l|l|l|l|l|l|l|l|l|l|l|}
\hline Studienjahr & 1 & 2 & 3 & 4 & 5 & 6 & 7 & 8 & 9 & 10 \\
\hline Wochenstunden & & & & & & & & & & \\
\hline
\end{tabular}

b) Falls Sie im Laufe Ihres Studiums erwerbstätig waren, was war bzw. ist der Hauptgrund für Ihre Erwerbstätigkeit(en)? (bitte nur eine Nennung)

Existenzsicherung

Zusatzeinkommen

Verbesserung der Berufschancen 
12. Im Zuge einer Befragung der ÖH (1998) gab der Großteil der befragten WUStudierenden an, daß sich einige selektive Prüfungen verlangsamend auf die Studienzeit auswirken. Beantworten Sie bitte die folgenden Fragen aufgrund Ihrer eigenen Erfahrung.

(1) Welche Lehrveranstaltungen des ersten Abschnittes wirken sich Ihrer Meinung nach verlangsamend auf die Studiendauer aus?

(2) Welche Diplom- bzw. Vorprüfungen des ersten Abschnittes wirken sich verlangsamend auf die Studiendauer aus?

(3) Welche Lehrveranstaltungen des zweiten Abschnittes wirken sich verlangsamend auf die Studiendauer aus?

(4) Welche Diplom-bzw. Vorprüfungen des zweiten Abschnittes wirken sich verlangsamend auf die Studiendauer aus?

13. Welche Maßnahmen würden Sie - Ihren Erfahrungen an der WU entsprechend - der Universitätsleitung vorschlagen, um die durchschnittliche Studiendauer und die Abbruchquote zu verringern?

\section{Danke für Ihre Mitarbeit!}




\section{Abbildungsverzeichnis}

3.1 Beispiel von Typ I zensierten $\left(C_{1}\right.$ und $\left.C_{3}\right)$, zufällig zensierten Zeiten $\left(C_{2}\right.$ und $\left.C_{4}\right)$ und Ereigniszeiten $T_{i} \ldots \ldots \ldots$

5.1 Dichte- (f), Überlebens- (S) und Hazardfunktion (h) des ExponentialModells für $\lambda=0.2 \ldots \ldots \ldots \ldots \ldots$

5.2 Hazardraten des Weibull-Modells für $\lambda=0.2$ und $\sigma=0.25\left(h_{1}\right)$, $\sigma=0.5\left(h_{2}\right), \sigma=0.8\left(h_{3}\right), \sigma=1.5\left(h_{4}\right) \ldots \ldots \ldots \ldots$

5.3 Hazardraten des log-normalen Modells für $\mu=0$ und $\sigma=0.5\left(h_{1}\right)$, $\sigma=1\left(h_{2}\right), \sigma=1.5\left(h_{3}\right) \ldots \ldots \ldots \ldots \ldots \ldots$

5.4 Hazardraten des log-logistischen Modells für $\lambda=0.2$ und $\sigma=$ $0.5\left(h_{1}\right), \sigma=1\left(h_{2}\right), \sigma=2\left(h_{3}\right) \ldots \ldots \ldots \ldots . \ldots . \ldots 48$

5.5 Hazardraten des verallgemeinerten Gamma-Modells für $\mu=0$, $\sigma=1$ und $\kappa=0.5\left(h_{1}\right), \kappa=0.9\left(h_{2}\right), \kappa=1.5\left(h_{3}\right), \kappa=10\left(h_{4}\right) \ldots .50$

5.6 Hazardraten des Gompertz-Modells für $\exp \left(\theta_{0}\right)=1$ und $\gamma=$ $0.2\left(h_{1}\right), \gamma=0.05\left(h_{2}\right), \gamma=-0.5\left(h_{3}\right) \ldots \ldots \ldots \ldots 1$

5.7 Proportionale Hazardraten der Weibull-Verteilung . . . . . . . . 52

12.1 Graphische Überprüfung der log-logistischen Verteilungsannahme für die Reststudiendauer ("ldur" bezeichnet die logarithmierte Reststudiendauer $t_{1}$ )

12.2 Geschätzte Hazardrate für den Studienabschluss bei log-logistischer Verteilungsannahme (ohne Einbeziehung von Kovariablen) . . . . 152

12.3 Plot der (geglätteten) Martingal-Residuen gegen die Variable "lag" (in Monaten; log-logistisches Modell) . . . . . . . . . . . . . 154

12.4 Plot der (geglätteten) Martingal-Residuen gegen die Variable "matalt" (log-logistisches Modell) . . . . . . . . . . . 155 
12.5 Plot der Cox-Snell Residuen bei Implementierung eines loglogistischen Modells für den Studienabschluss . . . . . . . . . 157

12.6 Plot der Cox-Snell Residuen bei Implementierung eines ExponentialModells für den Studienabschluss . . . . . . . . . . . . . 158

12.7 Plot der (geglätteten) Martingal-Residuen gegen die Variable "gesposj1" (log-logistisches Modell) . . . . . . . . . . . 165

12.8 Plot der (geglätteten) Martingal-Residuen gegen die Variable "average" (log-logistisches Modell) . . . . . . . . . . . . 166

12.9 Plot der (geglätteten) Martingal-Residuen gegen die Variable "gesposj1" (Cox-Modell) . . . . . . . . . . . . . . . . . . . 172

12.10 Plot der geschichteten (Kaplan-Meier-) Überlebensfunktionen für die binäre Variable "vpj1" (Studiendauer in Monaten) . . . . . 173

12.11 Plot der geschichteten (Kaplan-Meier-) Überlebensfunktionen für die gruppierte Variable "posj1" . . . . . . . . . . . . . . 174

12.12 Plot der geschichteten (Kaplan-Meier-) Überlebensfunktionen für die gruppierte Variable "av" . . . . . . . . . . . . . . . 175

12.13 Plot der geschichteten (Kaplan-Meier-) Überlebensfunktionen für die binäre Variable "englisch34" . . . . . . . . . . . . 180

13.1 Plot der (geglätteten) Martingal-Residuen gegen die Variable "gesposj1" im übergangsspezifischen Cox-Modell für den Studienabbruch . . . . . . . . . . . . . . . . 194 


\section{Tabellenverzeichnis}

10.1 Vergleich der Definitionen der Studiendauer entsprechend den Definitionen (a), (b) und (c) für die befragten Studierenden . . . . 125

11.1 Studienfortschritt und Studierstatus der Jahrgänge 1990 und 1993

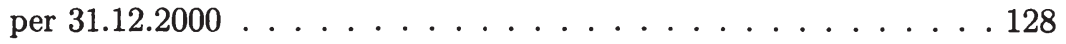

11.2 Studienfortschritt der System-Abbrecher und der Studierenden per

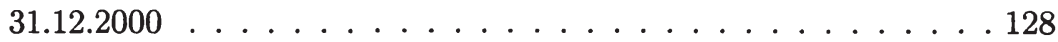

11.3 Leistungen der System-Abbrecher und nicht-aktiven Studierenden . . . . . . . . . . . . . . . . . 129

11.4 Leistungsprofil der Jahrgänge 1990 und 1993 nach Studierstatus . 130

11.5 Verteilung der Stichprobe nach Studierstatus und Jahrgang . . . . 131

11.6 Abschlussabsicht der Studienabbrecher zu Studienbeginn . . . . . 132

11.7 Verteilung der Studienabbrecher nach Hauptaktivität nach dem Studienabbruch und ihr Bezug zum WU-Studium . . . . . . 133

11.8 Verteilung der Studienabbrecher nach Hauptaktivität im Jahr nach dem Studienabbruch und dem Zeitpunkt des Abbruchs . . . . . . . . . . . . . 134

11.9 Verteilungen der untersuchten Population und der StichprobenPopulation nach den Variablen Geschlecht, Alter und Vorbildung . 135

11.10 Verteilung des Jahrgangs 1993 nach Vorbildung und Studierstatus

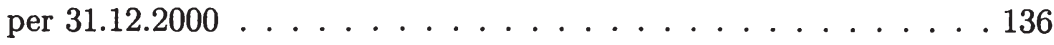

11.11 Verteilung der Stichproben-Population nach der Schul-Abschlussnote in Mathematik und dem Studierstatus per 31.12.2000 . . . . . . 138

11.12 Verteilung der Stichproben-Population nach der Schul-Abschlussnote in Englisch und dem Studierstatus per 31.12.2000 . . . . . . . . 139 
11.13 Verteilung der Stichproben-Population nach dem Besuch einer zweiten Ausbildung und dem Studierstatus per 31.12.2000 . . . . 140

11.14 Verteilung der Studierenden mit paralleler Ausbildung nach der Art der zweiten Ausbildung und dem Bezug zum WU-Studium . . 140

11.15 Häufigkeitsverteilung der Studierenden mit paralleler Ausbildung nach der Anzahl der für diese Ausbildung beanspruchten Semester . . . . . . . . . . . . . . . . . . 141

11.16 Verteilung der Stichproben-Population nach dem Absolvieren eines Auslandssemesters und dem Studierstatus per 31.12.2000 . . . . 142

11.17 Verteilung der Stichproben-Population nach dem Nachgehen einer Erwerbstätigkeit und dem Studierstatus per 31.12.2000 . . . . . 143

11.18 Verteilung der Stichproben-Population nach dem Erwerbstätigkeitsstatus im ersten Semester und dem Studierstatus per 31.12.2000 . 144

11.19 Durchschnittliche Berufsintensität (in Wochenstunden) während der WU-Studienzeit nach Studierstatus . . . . . . . . . . . . 144

11.20 Verteilung der Berufstätigen nach dem (vorwiegenden) Grund für die Erwerbstätigkeit und dem Studierstatus per 31.12.2000 . . . 145

11.21 Verteilung der Stichproben-Population nach der Betreuung von Kindern und dem Studierstatus per 31.12.2000 . . . . . . . . . 146

12.1 Log-Likelihood- und $A I C$-Werte für verschiedene Verteilungsannahmen für die Reststudiendauer . . . . . . . . . . . . . 149

12.2 Parameterschätzer des log-logistischen Modells für die Reststudiendauer auf Basis der Stammdaten (Jahrgang 1990) . . . . . . 156

12.3 Parameterschätzer unter Berücksichtigung von Interaktionvariablen mit dem Geschlecht (Jahrgang 1990; log-logistisches Modell) . 159

12.4 Parameterschätzer des finalen log-logistischen Modells für die Reststudiendauer auf Basis der Stammdaten (Jahrgang 1990) . . . . 160

12.5 Parameterschätzer des log-logistischen Modells der Reststudiendauer für die Jahrgänge 1989 bis 1993 auf Basis der Stammdaten . 163

12.6 Parameterschätzer der binären Jahrgangs-Variablen (Jahrgänge 1989 bis 1993) . . . . . . . . . . . . . . . . . . . 164 
12.7 Analyse der medianen Reststudiendauer der Jahrgänge 1989 bis 1993 auf Basis der Stamm- und Prüfungsdaten (log-logistisches Modell) . . . . . . . . . . . . . . . . 167

12.8 Parameterschätzer eines semiparametrischen Cox-Modells für den Studienabschluss auf Basis der Stammdaten der Jahrgänge 1989 bis 1993

12.9 Parameterschätzer eines semiparametrischen Cox-Modells für den Studienabbschluss auf Basis der Stamm- und Prüfungsdaten (Jahrgänge 1989 bis 1993) . . . . . . . . . . . . . . . . 176

12.10 Parameterschätzer eines semiparametrischen Cox-Modells für den Studienabschluss für die Jahrgänge 1990 und $1993 \ldots$. . . . . 178

12.11 Parameterschätzer eines semiparametrischen Cox-Modells für den Studienabschluss auf Basis einer Stichprobe der Jahrgänge 1990

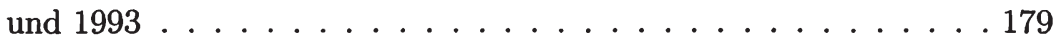

12.12 Parameterschätzer eines semiparametrischen Cox-Modells für den Studienabschluss unter ergänzender Berücksichtigung von zeitun abhängigen Variablen aus der Befragung . . . . . . . . . . 181

12.13 Parameterschätzer eines semiparametrischen Cox-Modells für den Studienabschluss unter Berücksichtigung der erhobenen zeitabhängigen Variablen . . . . . . . . . . . . . . . 184

13.1 Parameterschätzer des logistischen Regressionsmodells für den Studienabbruch ohne positives Prüfungsergebnis (Jahrgänge 1989 bis

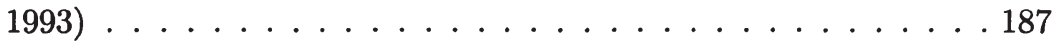

13.2 Parameterschätzer eines logistischen Regressionsmodells für den Studienabbruch ohne positive Leistung auf Basis der StichprobenPopulation . . . . . . . . . . . . . . . . . 190

13.3 Parameterschätzer eines logistischen Regressionsmodells für den Studienabbruch ohne positive Leistung unter Berücksichtigung von ergänzenden Variablen aus der Befragung . . . . . . . . . . 191

13.4 Analyse der Studienabbruchrate auf Basis der Stammdaten aus der Hörerevidenz (Jahrgänge 1989 bis 1993) . . . . . . . . . . 193

13.5 Analyse der Studienabbruchrate auf Basis der Stamm- und Prüfungsdaten aus der Hörerevidenz . . . . . . . . . . . . . . . 195 
13.6 Parameterschätzer des semiparametrischen Cox-Modells für den Studienabbruch unter Berücksichtigung von ergänzenden Variablen aus der Befragung . . . . . . . . . . . . . . . 197

13.7 Parameterschätzer des semiparametrischen Cox-Modells für den Studienabbruch unter Berücksichtigung von zeitabhängigen Variablen (Stichproben-Population) . . . . . . . . . . . . 198

14.1 Sensitivitätsanalyse des log-logistischen Modells für die Reststudiendauer mit modifizierten Studienabbruch-Zeiten (Jahrgänge 1989 bis 1993) . . . . . . . . . . . . . . . . . . 200

14.2 Analyse des zweiten Studienabschnitts anhand von links abgeschnittenen Daten (Stichproben-Population) . . . . . . . 202

14.3 Analyse der Studienabbruch-Wahrscheinlichkeit auf Basis des gruppierten Cox-Modells . . . . . . . . . . . . . . . 203 


\section{Literaturverzeichnis}

O.O. Aalen. Nonparametric inference for a family of counting processes. Annals of Statistics, 6:701-726, 1978.

H. Akaikie. A new look at statistical model identification. IEEE Transactions on Automatic Control, 19:716-723, 1974.

J.H. Aldrich und F.D. Nelson. Linear Probability, Logit, and Probit Models. Sage Publications, London, 1987.

P.D. Allison. Discrete-time methods for the analysis of event histories. In S. Leinhardt, editor, Sociological Methodology, 1982.

P.D. Allison. Event History Analysis. Sage Publications, London, 1984.

P.D. Allison. Survival Analysis using the SAS System. SAS, North Carolina, 1998.

P.K. Andersen, O. Borgan, R.D Gill, und N. Keiding. Statistical Models based on Counting Processes. Springer, New York, 1993.

W.B. Bilker und M. Wang. A semiparametric extension of the Mann-Whitney test for randomly truncated data. Biometrics, 52:10-20, 1996.

H.P. Blossfeld, A. Hamerle, und K.U. Mayer. Ereignisanalyse - Statistische Theorie und Anwendungen in den Wirtschafts- und Sozialwissenschaften. Campus Studium, Frankfurt/Main, New York, 1986.

H.P. Blossfeld und G. Rohwer. Techniques of Event History Modeling. Lawrence Erlbaum Associates, New Jersey, 1995.

A.L. Booth und S. Satchell. The hazards of doing a $\mathrm{PhD}$ : an analysis of completition and withdrawal rates of British $\mathrm{PhD}$ students in the 1980s. Journal of the Royal Statistical Society, Ser. A, 158:297-318, 1995. 
N.E. Breslow. Covariance analysis of censored survival data. Biometrics, 30: 89-100, 1974.

W. S. Cleveland. Robust locally weighted regression and smoothing scatter plots. Journal of the American Statistical Association, 74:829-836, 1979.

D. Collett. Modelling Survival Data in Medical Research. Chapman and Hall, London, 1994.

D.R. Cox. Regression models and life tables. Journal of the Royal Statistical Society, Ser. B, 34:187-220, 1972.

D.R. Cox und D. Oakes. Analysis of Survival Data. Chapman and Hall, London, 1984.

N.R. Draper und H. Smith. Applied Regression Analysis. Wiley \& Sons, New York, 1981.

B. Efron. The efficiency of Cox's likelihood function for censored data. Journal of American Statistical Association, 72:557-565, 1977.

T.R. Fleming und D.P. Harrington. Counting Processes and Survival Analysis. Wiley, New York, 1991.

P.M. Grambsch und T.M. Therneau. Proportional hazards tests in diagnostics based on weighted residuals. Biometrika, 81:515-526, 1994.

P.M. Grambsch, T.M. Therneau, und T.R. Fleming. Diagnostic plots to reveal functional form for covariates in multiplicative intensity models. Biometrics, 51:1469-1483, 1995.

M. Greenwood. The natural duration of cancer. In Reports on public health and medical subjects, 1926.

W. Grossmann, M. Hudec, und R. Kurzawa. Ergebnisse der empirischen Studie „,Gründe und Ursachen für die langen Studienzeiten in Österreich”. Institut für Statistik, Operations Research und Computerverfahren, Wien, 1999a.

W. Grossmann, M. Hudec, und R. Kurzawa. Tabellenband für die Studiengruppen Betriebswirtschaftslehre, Handelswissenschaften und BWZ. Institut für Statistik, Operations Research und Computerverfahren, Wien, 1999b.

D.S. Hachen. The competing risks model. Sociological Methods $\&$ Research, 17:21-54, 1988. 
P. Hackl und G. Sedlacek. Forschungsprojeht Studienverlaufsanalyse. Projektbericht, Wirtschaftsuniversität Wien, 2002.

A. Hamerle. Multiple-spell regression models for duration data. Applied Statistics, 38:127-138, 1989.

A. Hamerle und G. Tutz. Diskrete Modelle zur Analyse von Verweildauer und Lebenszeiten. Campus Forschung, Frankfurt/Main, New York, 1989.

F.E. Harrell. Predicting Outcomes: Applied Survival Analysis and Logistic Regression. University of Virginia, Charlottesville VA, 1997.

F.E. Harrell und R. Goldstein. A survey of microcomputer survival analysis software: The need for an integrated framework. The American Statistican, 51:360-373, 1997.

J. Häfke-Schönthaler. Zur Problematik der universitären Studieneingangsphase. Dissertation, Wirtschaftsuniversität Wien, 1999.

W. Hoffmann. Studienverlaufsanalyse für die Wirtschaftsuniversität Wien. Diplomarbeit, Wirtschaftsuniversität Wien, 1987.

D.W. Hosmer und S. Lemeshow. Applied Survival Analysis. Wiley, New York, 1999.

P. Hougaard. Fundamentals of survival data. Biometrics, 55:13-22, 1999a.

P. Hougaard. Multi-state models: A review. Lifetime Data Analysis, 5:239-264, 1999b.

J.D. Kalbfleisch und R.L. Prentice. Marginal likelihoods based on Cox's regression and life model. Biometrika, 60:267-278, 1973.

J.D. Kalbfleisch und R.L. Prentice. The statistical Analysis of Failure Time Data. John Wiley ans Sons, New York, 1980.

E.L. Kaplan und P. Meier. Nonparametric estimation from incomplete observations. Journal of the American Statistical Association, 53:457-481, 1958.

J.P. Klein und P.K. Goel. Survival Analysis: State of the Art. Kluwer Academic Publishers, Dordrecht, 1992.

J.P. Klein und M.L. Moeschberger. Survival Analysis, Techniques for Censored and Truncated Data. Springer, New York, 1997. 
D.G. Kleinbaum. Survival Analysis: A Self-Learning Text. Springer, New York, 1996.

S.W. Lagakos. General right censoring and its impact on the analysis of survival data. Biometrics, 35:139-156, 1979.

T. Lancaster. The Econometric Analysis of Transition Data. Econometric Society Monographs, Cambridge, 1990.

J.F. Lawless. Inference in the generailzed gamma and log gamma distributions. Technometrics, 22:409-419, 1980.

J.F. Lawless. Statistical Models and Methods for Lifetime Data. John Wiley and Sons, New York, 1982.

C.T. Le. Applied Survival Analysis. John Wiley and Sons, New York, 1997.

E.T. Lee. Statistical Methods for Survival Data Analysis. John Wiley and Sons, New York, 1992.

D.Y. Lin und T.R. Fleming. Proceedings of the First Seattle Symposium in Biostatistics: Survival Analysis. Springer, New York, 1997.

G. Mautner und G. Brandenburg. Auslandserfahrungen von Absolvent/inn/en der WU. wu-memo, 36:71-72, 2001.

W. Nelson. Theory and applications of hazard plotting for censored failure data. Technometrics, 14:945-965, 1972.

D. Oakes. Model-based and/or marginal analysis for multiple event-time data. In D.Y. Lin und T.R. Fleming, editors, Proceedings of the first Seattle Symposium in Biostatistics: Survival Analysis, 1997.

T. Petersen. Fitting parametric survival models with time-dependent covariates. Applied Statistics, 35:281-288, 1986.

T. Petersen. Analyzing event histories. In Statistical methods in longitudinal research, 1990.

L.R. Prentice und J.D. Kalbfleisch. Hazard rate models with covariates. Biometrics, 35:25-39, 1979.

L.R. Prentice, J.D. Kalbfleisch, A.V. Peterson, N. Flournoy, V.T. Farewell, und N.E. Breslow. The analysis of failure times in the presence of competing risks. Biometrics, 34:541-554, 1978. 
L.R. Prentice, B.J. Williams, und A.V. Peterson. On the regression analysis of multivariate failure time data. Biometrika, 68:373-379, 1981.

G. Rohwer und U. Pötter. TDA User's Manual. Ruhr-Universität Bochum, Bochum, 1998.

W. Schneider und R. Weiländer. Ist eine Entlastung der Wirtschaftsuniversität durch ein verbessertes Drop-Out-Mamgement möglich. Projektbericht, Abteilung für Wirtschaftspädagogik an der Wirtschaftsuniversität Wien, 1990.

G. Shumeli und A. Cohen. Analysis and display of hierarchical life-time data. The American Statistican, 53:140-146, 1999.

J.P. Smith und A.N. Naylor. Dropping out of university: a statistical analysis of the probability of withdrawal for UK university students. Journal of the Royal Statistical Society, 2001.

T.M. Therneau. Extending the Cox model. In D.Y. Lin und T.R. Fleming, editors, Proceedings of the first Seattle Symposium in Biostatistics: Survival Analysis, 1997.

T.M. Therneau, P.M. Grambsch, und T.R. Fleming. Martingal-based residuals for survival models. Biometrika, 77:147-161, 1990.

Toutenburg. Deskriptive Statistik. Springer, Berlin New Vork, 2000a.

Toutenburg. Induktive Statistik. Springer, Berlin New Vork, 2000b.

N.B. Tuma und M.T. Hannan. Social Dynamics - Models and Methods. Academic Press, Orlando, 1984.

J.K. Vermunt. Log-Linear Models for Event Histories. Sage Publications, London, 1997.

A. Wangler. Heterogenitätsprobleme in der Verlaufsdatenanalyse. Peter Lang, Frankurt/Main, 1997.

L.J. Wei, D.J. Lin, und L. Weissfeld. Regression analysis of multivariate incomplete failure time data by modeling marginal didtributions. Journal of the American Statistical Association, 84:1065-1073, 1989.

A. Würger. Kenngrößen der Studiumsstrukturen eines wirtschaftswissenschaftlichen Fachbereiches. Peter Lang, Frankurt/Main, 1992.

K. Yamaguchi. Event History Analysis. Sage Publications, London, 1991. 


\section{Forschungsergebnisse der Wirtschaftsuniversität Wien}

Herausgeber: Wirtschaftsuniversität Wien vertreten durch a.o. Univ. Prof. Dr. Barbara Sporn

Band 1 Stefan Felder: Frequenzallokation in der Telekommunikation. Ökonomische Analyse der Vergabe von Frequenzen unter besonderer Berücksichtigung der UMTS-Auktionen. 2004.

Band 2 Thomas Haller: Marketing Im liberalisierten Strommarkt. Kommunikation und Produktplanung im Privatkundenmarkt. 2005.

Band 3 Alexander Stremitzer: Agency Theory: Methodology, Analysis. A Structured Approach to Writing Contracts. 2005.

Band 4 Günther Sedlacek: Analyse der Studiendauer und des Studienabbruch-Risikos. Unter Verwendung der statistischen Methoden der Ereignisanalyse. 2004.

www.peterlang.de 